\title{
PREDICTING FLUID
}

RESPONSIVENESS IN ICU PATIENTS From physiology to bedside

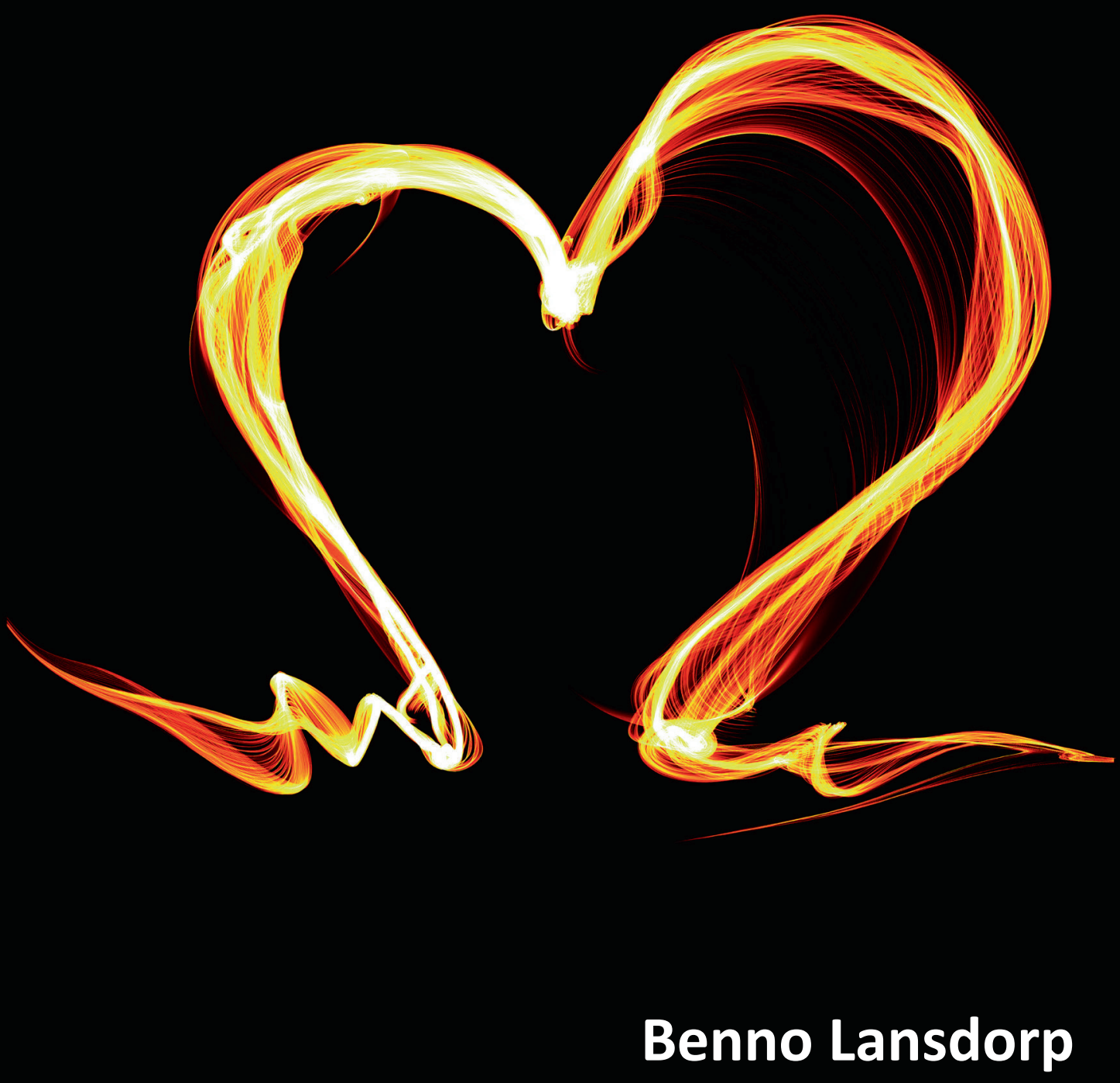





\section{PREDICTING FLUID RESPONSIVENESS IN ICU PATIENTS FROM PHYSIOLOGY TO BEDSIDE}

\section{Benno Lansdorp}


The research described in this thesis has been conducted within a collaboration of the educational program of Technical Medicine, which is part of the Faculty of Science and Technology at the University of Twente, and the department of Intensive Care Medicine at the Radboud University Medical Center.

Nederlandse titel:

Voorspellen van volume responsiviteit bij IC patienten - van fysiologie naar kliniek

Publisher:

Benno Lansdorp, University of Twente, P.O.Box 217, 7500AE Enschede, The Netherlands http://www.utwente.nl/ b.lansdorp@utwente.nl

Cover illustration: Shutterstock photographs

Printed by: Gildeprint Drukkerijen - Enschede

ISBN: 978-90-365-3670-7

DOI: http://dx.doi.org/10.3990/1.9789036535991

(c) Benno Lansdorp, Arnhem, The Netherlands, 2014.

No part of this work may be reproduced by print, photocopy or any other means without the permission in writing from the publisher. 


\title{
PREDICTING FLUID \\ RESPONSIVENESS IN ICU PATIENTS \\ FROM PHYSIOLOGY TO BEDSIDE
}

\author{
PROEFSCHRIFT
}

ter verkrijging van

de graad van doctor aan de Universiteit Twente,

op gezag van de rector magnificus,

Prof. dr. H. Brinksma,

volgens besluit van het College voor Promoties

in het openbaar te verdedigen

op vrijdag 27 juni 2014 om 16.45 uur

door

Benno Lansdorp

geboren op 1 september 1982

te Amersfoort, Nederland 
Dit proefschrift is goedgekeurd door:

Prof.dr.ir. M.J.A.M. van Putten, promotor

Prof.dr. J.G. van der Hoeven, promotor

Dr. J. Lemson, assistent-promotor

Copyright (C) 2014 by B. Lansdorp, Arnhem, The Netherlands. ISBN: 978-90-365-3670-7 


\section{PhD dissertation committee:}

Chairman and Secretary:

Prof. dr. ir. J.W.M. Hilgenkamp

University of Twente, Enschede, the Netherlands

\section{Promotors:}

Prof. dr. ir. M.J.A.M. van Putten

Clinical Neurophysiology

University of Twente, Enschede, the Netherlands

Prof. dr. J.G. van der Hoeven

Intensive Care Medicine

Radboud University Medical Center, Nijmegen, the Netherlands Assistant-Promotor:

Dr. J. Lemson

Intensive Care Medicine

Radboud University Medical Center, Nijmegen, the Netherlands Referee:

Prof. dr. P. Pickkers

Intensive Care Medicine

Radboud University Medical Center, Nijmegen, the Netherlands

\section{Opponents:}

Prof. dr. ir. P.H. Veltink

Biomedical Signals and Systems

University of Twente, Enschede, the Netherlands

Dr. ir. F.H.C. de Jongh

Bio-Fluid Dynamics

University of Twente, Enschede, the Netherlands

Prof. dr. J. Bakker

Intensive Care Medicine

Erasmus Medical Center, Rotterdam, the Netherlands

Dr. J.J. Maas

Intensive Care Medicine

Leiden University Medical Center

\section{Paranymphs:}

Bart Lansdorp

Tobias Los 

This thesis is dedicated to my mother, my biggest fan. 



\section{Contents}

List of Abbreviations $\quad$ xi

$\begin{array}{ll}\text { Introduction } & 1\end{array}$

1 Introduction 3

Part I Dynamic indices and fluid responsiveness $\quad 10$

2 Predicting fluid responsiveness in the ICU 13

3 Dynamic indices do not predict volume responsiveness in routine clinical practice

4 Ventilator induced pulse pressure variation in neonates

Part II: Intra-thoracic pressure distribution during mechanical ventilation in health and disease

5 Mechanical ventilation-induced intra-thoracic pressure distribution and heart-lung interactions

6 Intra-thoracic pressure distribution during mechanical ventilation in a newborn animal model: influence of tidal volume and progressive ARDS 85

7 Dynamic preload indicators decrease when the abdomen is opened 103

8 A mathematical model for the prediction of fluid responsiveness 115 


\section{Part III: Non-invasive techniques to assess fluid respon-} siveness

9 Validation of non-invasive pulse contour cardiac output using finger arterial pressure in cardiac surgery patients requiring fluid therapy

10 Non-invasive measurement of pulse pressure variation and systolic pressure variation using a finger cuff corresponds with intra-arterial measurement

11 Non-invasive determination of fluid responsiveness using dynamic indices and the passive leg-raising test

Summary, general discussion and future perspectives $\quad 184$

12 Summary, general discussion and future perspectives

13 Samenvatting in het Nederlands

Dankwoord

Curriculum vitae

List of publications

Appendix

Simulation instructor saved by student 


\section{List of Abbreviations}

$\begin{array}{ll}\text { ABP } & \text { Arterial Blood Pressure } \\ \text { AED } & \text { Automated External Defibrillator } \\ \text { AHA } & \text { Americal Heart Association } \\ \text { ALI } & \text { Acute Lung Injury } \\ \text { ANOVA } & \text { Analysis of Variance } \\ \text { AP } & \text { Arterial Pressure } \\ \text { ARDS } & \text { Adult Respiratory Distress Syndrome } \\ \text { BLS } & \text { Basic Life Support } \\ \text { BMI } & \text { Body Mass Index } \\ \text { BR } & \text { Baroreceptor } \\ \text { Brach } & \text { Brachial } \\ \text { C } & \text { Compliance } \\ \text { CABG } & \text { Coronary Artery Bypass Grafting } \\ \text { CI } & \text { Cardiac Index } \\ \text { CO } & \text { Cardiac Output } \\ \text { contr } & \text { Contractility } \\ \text { CPR } & \text { Cardio Pulmonary Resuscitation } \\ \text { CVP } & \text { Central Venous Pressure } \\ \text { e } & \text { Elastance } \\ \text { ECG } & \text { Electrocardiogram } \\ \text { f } & \text { Flow } \\ \text { FAP } & \text { Finger Arterial Pressure } \\ \text { FC } & \text { Fluid Challenge } \\ \text { Fing } & \text { Finger } \\ \text { Hb } & \text { Hemoglobin } \\ \text { HFV } & \text { High Frequency Ventilation } \\ \text { HR } & \text { Heart Rate } \\ \text { IAP } & \text { Intra Abdominal Pressure } \\ \text { ICD } & \text { Implantable Cardiac Defibrillator } \\ \text { ICU } & \text { Intensive Care Unit } \\ \text { ICV } & \text { Inferior Caval Vein }\end{array}$


ITP

L

LA

LOA

LV

MAP

NI

NIBP

$\mathrm{p}$

Paw

PCWP

PEEP

PEP

PLR

PP

Ppl

Ppc

PPV

Ptm

PVC

PVP

$\mathrm{R}$

RA

ROC

$\mathrm{RV}$

RR

SCV

SD

SE

SIMV

SPV

SV

SVI

SVR

SVV

$\mathrm{t}$

TD

TV

$\mathrm{v}$

$\mathrm{Vu}$
Intra Thoracic Pressure

Inertance

Left Atrium

Limits Of Agreement

Left Ventricle

Mean Arterial Pressure

Non-Invasive

Non-Invasive Blood Pressure

Pressure

Airway Pressure

Pulmonary Capillary Wedge Pressure

Positive End Expiratory Pressure

Pre-Ejection Period

Passive Leg Raising

Pulse Pressure

Pleural Pressure

Pericardial Pressure

Pulse Pressure Variation

Transmural Pressure

Premature Ventricular Contraction

Pressure Peripheral Vein

Resistance

Right Atrium / Right Atrial

Receiver Operator Characteristics

Right Ventricul(ar)

Respiratory Rate

Superior Caval Vein

Standard Deviation

Standard Error

Synchronized Intermittent Mandatory Ventilation

Systolic Pressure Variation

Stroke Volume

Stroke Volume Index

Systemic Vascular Resistance

Stroke Volume Variation

Time

Thermodilution

Tidal Volume

Volume

Unstressed Volume 


\section{Introduction}





\section{General introduction and outline of this thesis}




\subsection{Fluid: both essential and harmful}

Worldwide, more than 15 million patients are admitted to Intensive Care Units (ICUs) annually. In these patients, evaluation of the hemodynamic status is of upmost inportance, since circulatory insufficiency is very commonly encountered in the ICU [1]. Circulatory failure, as a result of a low cardiac output, may lead to inadequate tissue perfusion and oxygenation, the main functions of the cardiovascular system [2]. Since cardiac output is determined by the functioning of the pump (cardiac contractility), the tubing (vasomotor tone), and the fluid (intravascular volume), and uncorrected hypovolemia may increase organ hypoperfusion and ischemia, fluid loading is considered to be the first step in the resuscitation of hemodynamic instable patients [3]. Yet, multiple studies demonstrate that only about $50 \%$ of hemodynamically unstable ICU patients respond to a fluid challenge [4]. This is because the clinical determination of the intravascular volume can be extremely difficult in critically ill patients. In the other half of the patients, this overzealous fluid resuscitation, leading to excessive intravascular volume, can be futile and even deleterious [5-7]. For this reason, at the ICU, one constantly balances between hypovolemia and volume overload.

Circulatory failure as a result of hypovolemia can be distinguished in three different scenarios. The first scenario refers to patients admitted in the emergency room for evident acute body fluid losses. With this cause, together with the presence of clinical signs of hemodyanmic instability, the diagnosis of hypovolemia is almost certain and the patient will benefit from fluid therapy. The second scenario refers to patients with a high suspicion of severe sepsis or septic shock. Also in this category of patients, several studies emphasized the importance of volume resuscitation in the fist hours of management $[8,9]$. The third scenario refers to patients who have stayed in the ICU for several hours or days and who experience hemodynamic instability that requires urgent therapy. Volume administration may represent a therapeutic dilemma. On the one hand, one may expect a beneficial effect of fluid administration if the heart still has some preload reserve. On the other hand, because the patient has probably already been resuscitated, the presence of preload reserve is not guaranteed and further fluid infusion has the potential to promote a pulmonary edema, in particular in cases of increased pulmonary permeability. In this regard, positive cumulative fluid balance has been shown to be an independent risk of death [10]. In patients with acute lung injury, a restrictive fluid strategy was demonstrated to be better than a liberal fluid strategy in terms of ventilator-free and ICU-free days [11]. The resuscitation of critically ill patients, therefore, requires an accurate assessment of the patients' intravascular volume status. 


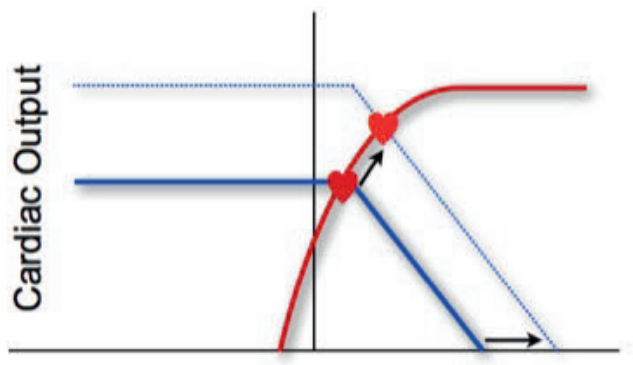

Preload

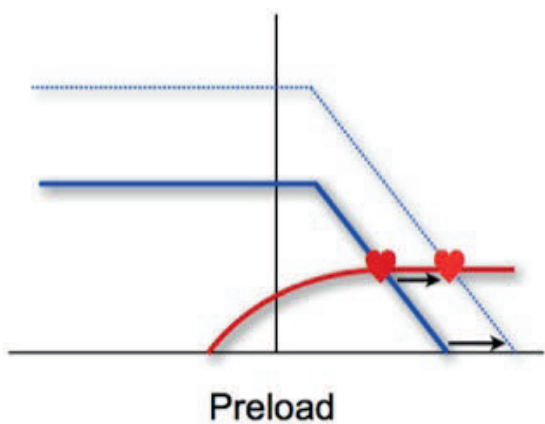

Preload

Figure 1: Representation of the cardiac function curve (red) and the venous return curve (blue). The cardiac output is determined by the interception of both curves (red heart symbol). Therefore, fluid infusion will only increase cardiac output when the heart is operating on the steep part of the cardiac function curve

\section{2 predictors of fluid responsiveness}

In order to identify patients who can benefit from fluid resuscitation by an increase in cardiac output, predictors of volume responsiveness are needed at the bedside. While cardiac output, together with the hemoglobine level and the saturation, determines tissue perfusion, a rise in cardiac output is central to the hemodynamic response to fluids. The prediction of fluid responsiveness is therefore defined as:

"the ability to predict a positive reaction in cardiac output to fluid administration based on a certain parameter"

Whether the cardiac output will increase after fluid expansion, is determined by the interaction of two functions: the cardiac (or Frank-Starling) function and venous return function. Fluid infusion, resulting in an increased preload (rightward shift of the venous return curve), will only increase cardiac output when the heart is operating on the steep part of the cardiac function curve (see Figure 1). For this reason, "upstream" indices of resuscitation such at blood pressure, and oxygen delivery do not provide adequate information for fluid resuscitation. Ultimately, the resuscitation of critically ill patients should be guided by "downstream" markers. Considering the Frank-Starling relationship, the response to volume infusion is more likely to occur when the ventricular preload is low than when it is high. For this reason, "static" markers of ventricular preload have been proposed to predict volume responsiveness. However, a given value of preload can be associated with either some preload reserve and hence volume responsiveness for a normal heart or with the absence of preload reserve in the case of a failing heart [12]. This causes that static markers, even in combination with clinical signs like hypotension, tachycardia, oliguria, mottled skin 

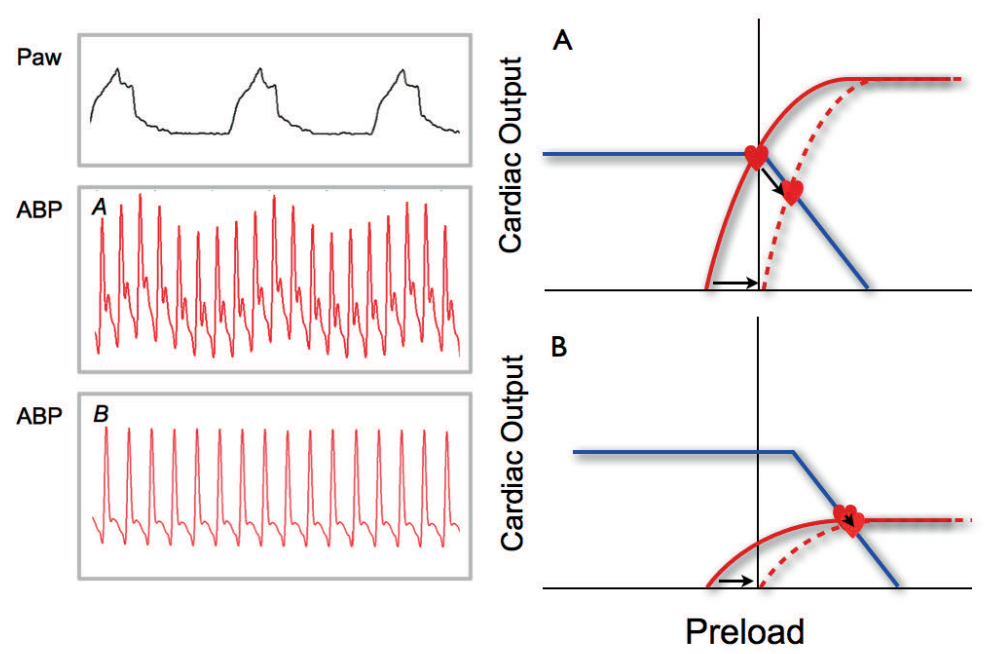

Figure 2: Intermitted positive airway pressure (Paw), shifting the cardiac function curve (red function) to the right, will result in arterial pressure variations (see ABP, A), indicating that the heart is functioning on the steep portion of the cardiac function curve and thus has "preload reserve". On the other hand, in patients operating on the flat portion of the cardiac function curve, the value of the dynamic indices will be low (see B).

or altered mental status, are not able to predict fluid responsiveness in hemodynamically unstable patients [13].

Over the last decade, a number of studies have reported the use of arterial variations in pressure and flow to assess fluid responsiveness. These variations, being a combination of upstream and downstream markers and quantified by so called dynamic indices like pulse pressure-, systolic pressure- or stroke volume-variation, arise a result of heart-lung interactions during mechanical ventilation [14]. Intermitted positive airway pressure, shifting the cardiac function curve to the right, will result in arterial pressure and flow variations, indicating that the heart is functioning on the steep portion of the cardiac function curve and thus has "preload reserve" or "recruitable" cardiac output. On the other hand, in patients operating on the flat portion of the cardiac function curve, positive pressure ventilation will not result in these kind of variations and the value of the dynamic indices will be low, see Figure 2 [15].

Although several studies have shown excellent accuracy for these dynamic indices to predict volume responsiveness, most of the study populations were strictly controlled, including e.g. controlled mechanical ventilation with no spontaneous breathing and tidal volumes above $7 \mathrm{ml} / \mathrm{kg}[16,17]$. Unfortunately, only few ICU 
patient meets this criteria in which dynamic indices have shown to be successful and there is still a lot of work to do to improve the reliability of dynamic indices in predicting fluid responsiveness [18].

\subsection{Outline of this thesis}

In the first part of this thesis, the clinical usefulness of dynamic indices is described. In particular, the way fluid responsiveness can be used in today's clinical practice, taking into considerations the clinical evidence (chapter 2). chapter 3 describes a critical analysis of the shortcommings of dynamic indices as used today and their implications for clinical usefulness. Additionally, the occurence and clinical usefullness of arterial pressure variations is studied in neonates (chapter 4).

The limited applicability of the dynamic indices regarding the prediction of fluid responsiveness in a wide range of ICU patients is partly caused by a lack of understanding of the exact origin of these arterial variations and the influence of some clinical variables. Therefore, the second part of this dissertation focuses on the physiological background of the heart lung interactions and dynamic indices. In chapter $\mathbf{5}$, the distribution of positive airway pressure in mechanically ventilated ICU patients is described in detail for healthy lungs, in a range of different clinical situations. Additionally, this distribution is also described for low compliant lungs, using a pediatric animal model that represents (prediatric) ICU patients at different stages of the respiratory distress syndrom (chapter 6). Besides the influence of differences in lung dynamics, also the effect of intra-abdominal pressure on dynamic indices is investigated (chapter 7). In chapter $\mathbf{8}$, a mathematical model is presented in which the physiology is combined. This model is able to simulated different mechanically ventilated ICU patients under various conditions and is intented to be used in the future to predict fluid responsivess at the bedside.

Finally, the third and last part of this thesis focuses on non-invasive techniques to assess hemodynamics and particularly fluid responsiveness (chapter 8-11).

Additionally, the appendix of this this thesis describes an amazing story in which the author of this thesis is the main character.

\section{References}

[1] M. R. Pinsky, "Hemodynamic evaluation and monitoring in the icu," Chest, vol. 132, no. 6, pp. 2020-9, 2007.

[2] D. L. Bredle and K. Reinhart, "Critical oxygen delivery in patients with sepsis," JAMA : the journal of the American Medical Association, vol. 271, no. 15, pp. 1158-9, 1994. 
[3] K. Murakawa and A. Kobayashi, "Effects of vasopressors on renal tissue gas tensions during hemorrhagic shock in dogs," Critical care medicine, vol. 16, no. 8, pp. 789-92, 1988.

[4] F. Michard and J. L. Teboul, "Predicting fluid responsiveness in icu patients: a critical analysis of the evidence," Chest, vol. 121, no. 6, pp. 2000-8, 2002.

[5] A. L. Rosenberg, R. E. Dechert, P. K. Park, and R. H. Bartlett, "Review of a large clinical series: association of cumulative fluid balance on outcome in acute lung injury: a retrospective review of the ardsnet tidal volume study cohort," $J$ Intensive Care Med, vol. 24, no. 1, pp. 35-46, 2009.

[6] S. Brandt, T. Regueira, H. Bracht, F. Porta, S. Djafarzadeh, J. Takala, J. Gorrasi, E. Borotto, V. Krejci, L. B. Hiltebrand, L. E. Bruegger, G. Beldi, L. Wilkens, P. M. Lepper, U. Kessler, and S. M. Jakob, "Effect of fluid resuscitation on mortality and organ function in experimental sepsis models," Crit Care, vol. 13, no. 6, p. R186, 2009.

[7] D. Payen, A. C. de Pont, Y. Sakr, C. Spies, K. Reinhart, and J. L. Vincent, "A positive fluid balance is associated with a worse outcome in patients with acute renal failure," Crit Care, vol. 12, no. 3, p. R74, 2008.

[8] E. Rivers, B. Nguyen, S. Havstad, J. Ressler, A. Muzzin, B. Knoblich, E. Peterson, and M. Tomlanovich, "Early goal-directed therapy in the treatment of severe sepsis and septic shock," The New England journal of medicine, vol. 345, no. 19, pp. 1368-77, 2001.

[9] M. Levy, W. Macias, J. Russell, M. Williams, B. Trzaskoma, E. Silva, and J. L. Vincent, "Failure to improve during first day of therapy is predictive of 28day mortality in severe sepsis," Chest, vol. 124, no. 4, 2004.

[10] M. J. Dubois, C. Orellana-Jimenez, C. Melot, D. De Backer, J. Berre, M. Leeman, S. Brimioulle, O. Appoloni, J. Creteur, and J. L. Vincent, "Albumin administration improves organ function in critically ill hypoalbuminemic patients: A prospective, randomized, controlled, pilot study," Critical care medicine, vol. 34, no. 10, pp. 2536-40, 2006.

[11] H. P. Wiedemann, A. P. Wheeler, G. R. Bernard, B. T. Thompson, D. Hayden, B. deBoisblanc, J. Connors, A. F., R. D. Hite, and A. L. Harabin, "Comparison of two fluid-management strategies in acute lung injury," The New England journal of medicine, vol. 354, no. 24, pp. 2564-75, 2006.

[12] D. Osman, C. Ridel, P. Ray, X. Monnet, N. Anguel, C. Richard, and J. L. Teboul, "Cardiac filling pressures are not appropriate to predict hemodynamic response to volume challenge," Crit Care Med, vol. 35, no. 1, pp. 64-8, 2007. 
[13] P. E. Marik, M. Baram, and B. Vahid, "Does central venous pressure predict fluid responsiveness? a systematic review of the literature and the tale of seven mares," Chest, vol. 134, no. 1, pp. 172-8, 2008.

[14] F. Feihl and A. F. Broccard, "Interactions between respiration and systemic hemodynamics. part i: basic concepts," Intensive Care Med, vol. 35, no. 1, pp. 45-54, 2009.

[15] W. T. McGee, "A simple physiologic algorithm for managing hemodynamics using stroke volume and stroke volume variation: physiologic optimization program," J Intensive Care Med, vol. 24, no. 6, pp. 352-60, 2009.

[16] P. E. Marik, R. Cavallazzi, T. Vasu, and A. Hirani, "Dynamic changes in arterial waveform derived variables and fluid responsiveness in mechanically ventilated patients: a systematic review of the literature," Crit Care Med, vol. 37, no. 9, pp. 2642-7, 2009.

[17] A. Kramer, D. Zygun, H. Hawes, P. Easton, and A. Ferland, "Pulse pressure variation predicts fluid responsiveness following coronary artery bypass surgery," Chest, vol. 126, no. 5, pp. 1563-8, 2004.

[18] S. Maguire, J. Rinehart, S. Vakharia, and M. Cannesson, "Technical communication: respiratory variation in pulse pressure and plethysmographic waveforms: intraoperative applicability in a north american academic center," Anesth Analg, vol. 112, no. 1, pp. 94-6, 2011. 


\section{Part I \\ Dynamic indices and fluid responsiveness}





\title{
5 \\ Predicting fluid responsiveness in the intensive care unit: a clinical guide
}

H. H. Woltjer, B. Lansdorp, M. Hilkens and J. G. van der Hoeven. Netherlands Journal of Critical Care 2009 Feb;13(1):31-7

\begin{abstract}
Fluid administration in critically ill patients is an important everyday therapeutic measure to improve organ perfusion. However, during the past decade, excessive fluid administration has been related to increased morbidity and mortality. This has led to the hypotheses that fluid administration without increasing cardiac output is inappropriate and is of no benefit to the patient. Over the past 10 years, many parameters for the prediction of fluid responsiveness have been suggested and validated. Implementation of these parameters in clinical practice may reduce the amount of inappropriate fluid. In this paper we discuss these methods for predicting fluid responsiveness and present a clinical strategy for fluid resuscitation. We make separate recommendations for patients on controlled mechanical ventilation, on mechanical ventilation with spontaneous activity and those breathing spontaneously.
\end{abstract}




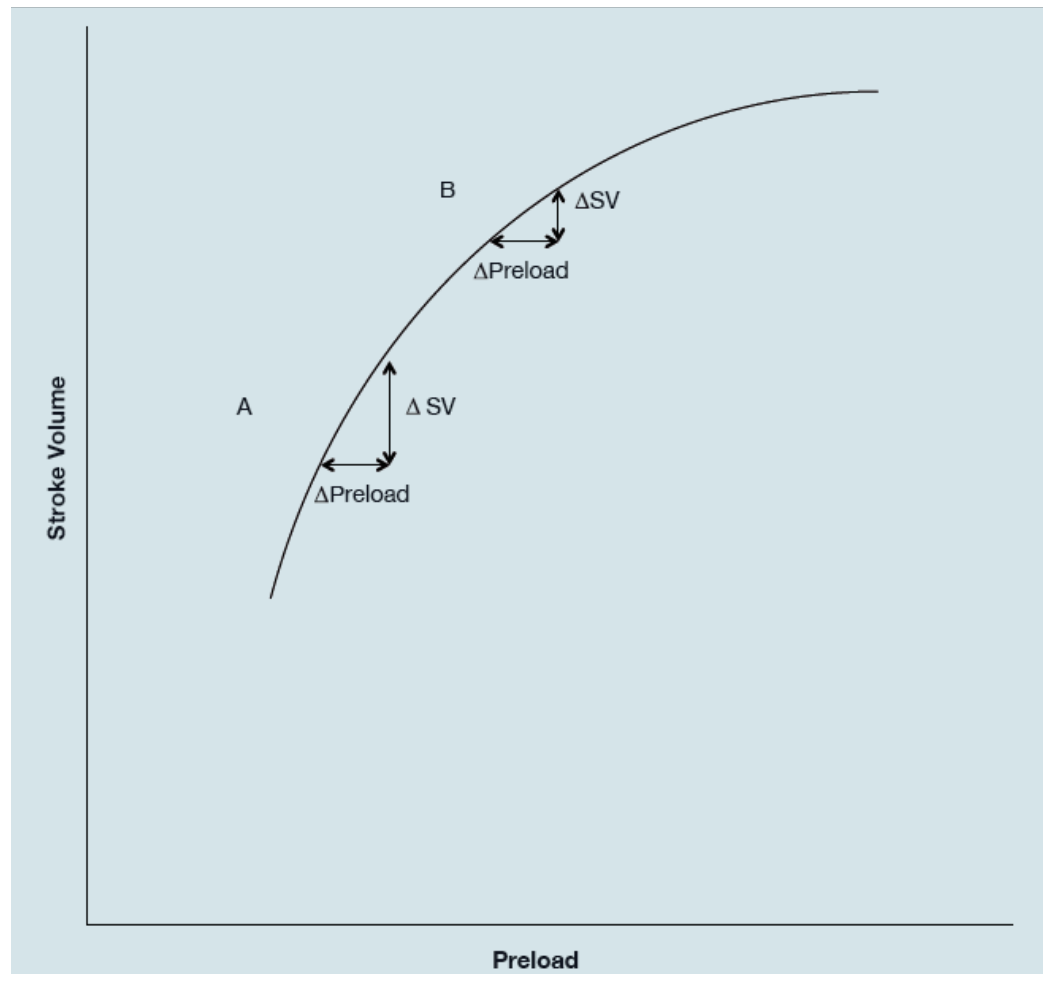

Figure 1: Preload change ( $\triangle$ Preload) is identical for situation $A$ and $B$, but stroke volume change $(\Delta S V)$ decreases moving up the Frank-Starling curve (A to B).

\subsection{Introduction}

Fluid resuscitation is one of the cornerstones to improve organ perfusion in patients with a critically compromised circulation. By increasing cardiac preload, fluid administration may increase cardiac output. When cardiac output increases as a result of fluid administration, the patient is considered to be fluid responsive. Excessive fluid resuscitation is associated with increased morbidity and mortality. In the presence of pulmonary oedema inappropriate fluid gain is associated with a worsened outcome [1,2]. The ARDS Network showed that conservative fluid management in patients with acute lung injury significantly shortened the duration of mechanical ventilation and of intensive care treatment [3].

In the past, optimal endpoints of fluid resuscitation have often relied on static indices such as blood pressure, central venous pressure (CVP) and pulmonary capillary wedge pressure (PCWP) [4]. However, nowadays the validity of static indices as a guide for fluid resuscitation is being questioned. Osman et al. [5] showed that a CVP 


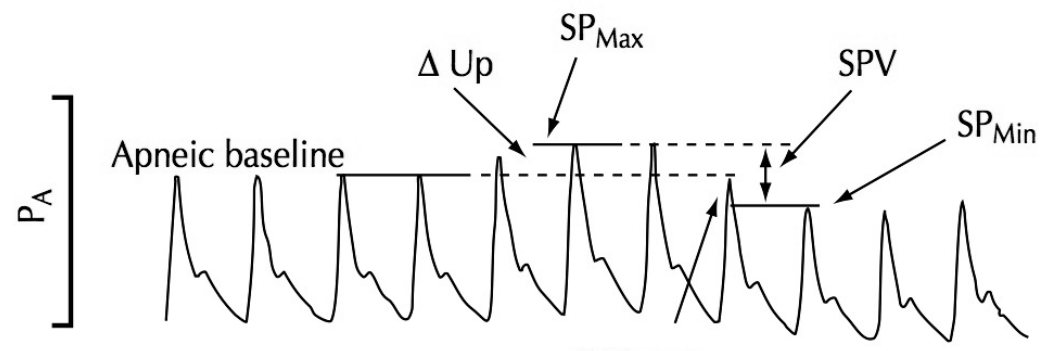

$\Delta$ Down

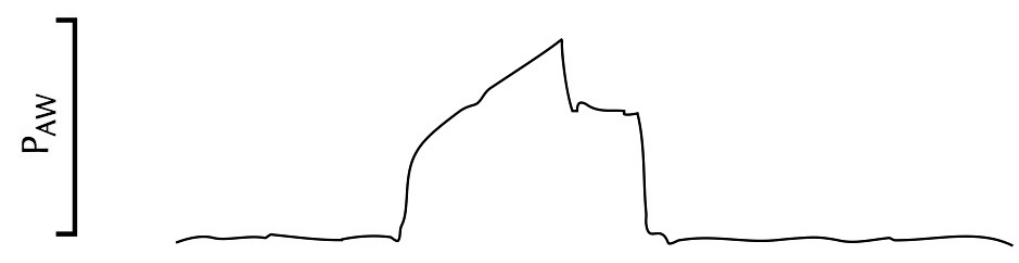

Figure 2: Systolic pressure variation (SPV) and delta down after an end-expiratory hold in a patient on pressure controlled ventilation. $P a=$ arterial pressure and Paw $=$ airway pressure

$<8 \mathrm{mmHg}$ and a PCWP $<12 \mathrm{mmHg}$ predicted fluid responsiveness with a positive predictive value of only $47 \%$ and $54 \%$, respectively. More recently, Marik et al [6] showed that the pooled correlation coefficient from 24 studies, between baseline CVP and a change in cardiac index was 0.18 (95\% CI, 0.08-0.28) with an area under the ROC curve of 0.56 (95\% CI, 0.51-0.61).

In the past 10 years many parameters have been proposed to predict and monitor fluid responsiveness. The accuracy of these methods has been established by their ability to predict an increase in cardiac index $>15 \%$. The purpose of this paper is to discuss these parameters for predicting fluid responsiveness in patients on controlled mechanical ventilation, mechanical ventilation with spontaneous activity and spontaneously breathing. 


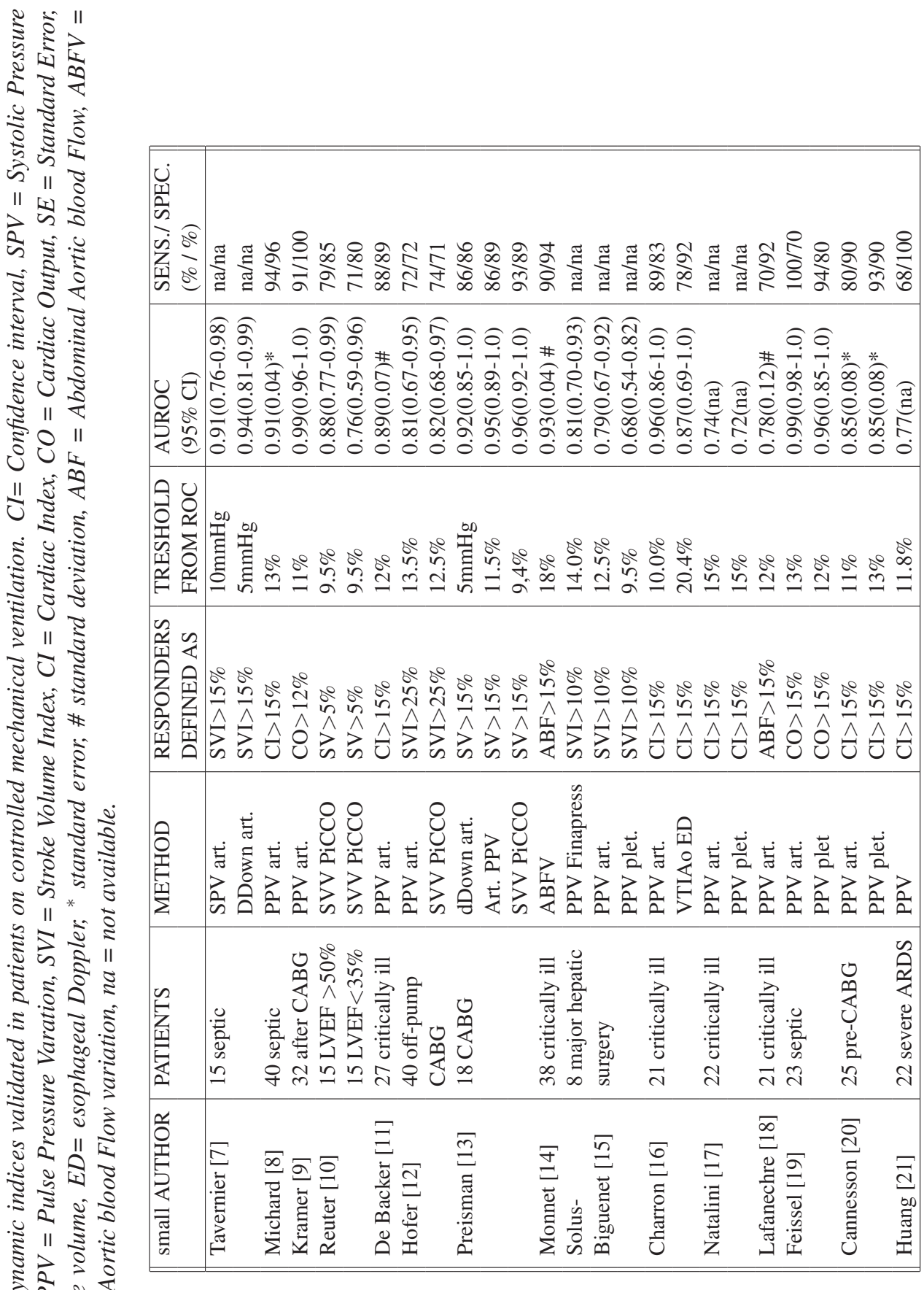




\subsection{Predicting fluid responsiveness in patients on controlled mechanical ventilation}

Positive pressure ventilation causes an intermittent change in preload of the heart. During inspiration the venous return to the right heart decreases thereby lowering preload and, in seconds is followed by a decrease in preload of the left heart. According to the Frank-Starling relationship, a decrease in preload results in a reduction of stroke volume. The magnitude of this effect depends on where the heart is operating on the Frank-Starling curve (Figure 1). If the heart is operating on the steep part of the curve this results in a significant change in stroke volume. If the heart moves higher up the curve, the change in stroke volume decreases. On the flat part of the curve stroke volume changes are minimal or absent. This heart-lung interaction during mechanical ventilation is the basis of the dynamic indices to predict fluid responsiveness such as stroke volume variation (SVV), and derivatives and the echocardiographic measurement of the caval vein collapsibility and distensibility.

\subsubsection{Measurement of dynamic indices}

Mechanical ventilation causes cyclic changes of left ventricular stroke volume and thereby cyclic changes of systolic pressure and pulse pressure. The increase in pleural pressure engendered by a mechanical breath causes a modest rise in arterial pressure (dUp), followed by a steady decrease (dDown). To measure dDown and dUp an end-expiratory hold must be performed to establish a baseline (Figure 2).

The augmentation of the arterial pressure at the onset of a mechanical breath has been explained by a temporary increase in left ventricular preload. The alveolar pressure squeezes the blood in the pulmonary capillaries towards the left atrium [22]. At the same time the transmural pressure of the left ventricle decreases due to an increase in pleural pressure effectively lowering afterload. A prominent dUp has been linked to an increase in afterload of the left ventricle and left ventricular failure $[23,24]$. In these situations temporary lowering of the afterload of the left ventricle may have a pronounced effect on cardiac output.

In 1987 Perel et al [25] showed in an animal model that dDown is closely related to graded haemorrhage and retransfusion. Tavernier et al [7] conducted the first clinical study in 15 patients with sepsis. This study showed that dDown and systolic pressure variation (SPV) were far better predictors of fluid responsiveness as compared to PCWP and echocardiographic left ventricular end diastolic area index. Today a substantial number of studies have confirmed these initial results in a variety of patient groups (Table 1).

SVV due to mechanical ventilation is the principal physiological explanation that predicts fluid responsiveness, while SPV and pulse pressure variation (PPV), are derivatives of SVV. Most studies use the arterial pressure reading, but the varia- 
tion in the amplitude of the plethysmographic pulse (Pplet), analogue to the arterial pressure reading, can also be used with comparable results [19]. Monnet et al [14] used oesophageal Doppler to trace stroke volume variation.

For adequate interpretation of SVV, PPV and SPV it is important to note that they require a regular heart rhythm and that they are influenced by tidal volume. De Backer et al [11] showed that PPV is only a reliable predictor of fluid responsiveness when a tidal volume equal or greater than $8 \mathrm{ml} / \mathrm{kg}$ is used. When a tidal volume $<8 \mathrm{ml} / \mathrm{kg}$ was used sensitivity and specificity for prediction of fluid responsiveness dropped from $88 \%$ to $39 \%$ and from $89 \%$ to $65 \%$, respectively. Lower tidal volumes may insignificantly affect pleural pressure and loading conditions of the left ventricle. Recently, however, Huang et al [21] used a low tidal volume strategy $(6.4 \pm 0.7 \mathrm{ml} / \mathrm{kg})$ with high PEEP $(13.9 \pm 1.4 \mathrm{~cm} \mathrm{H} 2 \mathrm{O})$, in 22 patients with severe ARDS and showed that a PPV $>11.8 \%$ predicted a positive response to volume expansion with a sensitivity of $68 \%$ and a specificity of $100 \%$. In the accompanying editorial, Michard et al [26] argued that PEEP induces an increase in mean airway pressure and pleural pressure causing a leftward shift on the Frank-Starling curve. Therefore, a patient operating on the flat part of the curve may move to the steep part and become fluid responsive. The relatively low sensitivity means that about one-third of patients who may benefit from a fluid challenge, are predicted not to. In an experimental animal model Kim et al [27] measured PPV at tidal volumes of 5,10,15 and $20 \mathrm{ml} / \mathrm{kg}$. PPV tended to increase with higher tidal volumes. Only a tidal volume of $20 \mathrm{ml} / \mathrm{kg}$ differed significantly $(\mathrm{p}<0.05)$ from the baseline tidal volume $(10 \mathrm{ml} / \mathrm{kg})$. From this study it was concluded that separate validation is required to define threshold pulse pressure. However, in clinical practice tidal volumes of $6-10 \mathrm{ml} / \mathrm{kg}$ are used. The threshold values for SVV and PPV for tidal volumes of $8-10 \mathrm{ml} / \mathrm{kg}$ have been validated (Table 1). For lower tidal volumes sensitivity will rapidly decrease [11,21], but specificity may remain high [21]. Another dynamic method that is used to predict fluid responsiveness in ventilated patients is the measurement of the endoluminal diameter change of the caval vein with echography. Mechanical ventilation causes fluctuations in blood flow to the right heart. This results in a cyclic change in the endoluminal diameter of the compliant inferior caval vein (ICV) and superior caval vein (SCV). The diameter of the ICV can be measured with trans-thoracic echography using the sub-xyphoidal long axis view, and from the minimum (Dmin) and maximum (Dmax) diameters a collapsibility or distensibility index can be calculated. Feissel et al. [28] studied 39 patients with septic shock on controlled mechanical ventilation and showed that a distensibility index of $>12 \%$ allowed identification of responders to a fluid challenge with a positive and negative predictive value of $93 \%$ and $92 \%$, respectively. The index was calculated as the difference between Dmax and Dmin, normalized by the mean of the two values, and expressed as a percentage. Barbier et al [29] used a slightly different calculation for the distensibility index (ratio of Dmax Dmin / Dmin 
expressed as a percentage). In 23 septic, mechanically-ventilated patients a threshold of $18 \%$ discriminated responders (increase in CI $>15 \%$ ) from non-responders with a sensitivity of $90 \%$ and a specificity of $90 \%$.

In only one study was the diameter of the SCV measured [30]. Measurement of the diameter of the SCV requires transoesophageal echocardiography (long axis view). In 66 septic, mechanically ventilated patients a collapsibility (Dmax-Dmin/Dmax expressed as a percentage) of $36 \%$ allowed discrimination between responders and non-responders with a sensitivity of $90 \%$ and a specificity of $100 \%$.

Since this method also depends on the interaction between mechanical ventilation and venous return it is likely to be influenced by the size of the tidal volume. In our experience, the cyclic fluctuation of the caval vein diameter indeed decreases when tidal volume is lowered. Appropriate training is needed for accurate measurement, although the diameter of the inferior caval vein is quite easy to determine. It is also unclear whether this method can be used in patients with an irregular heart rate as the flow in the caval vein is non-pulsatile. None of the current studies have specified this issue.

\subsection{Predicting fluid responsiveness in patients with sponta- neous breathing with or without mechanical support}

Until recently it was assumed that the dynamic indices were less useful for predicting fluid responsiveness in patients with spontaneous breathing activity because breathing frequency, tidal volume and the intrathoracic pressure are not controlled. However, spontaneous breathing also results in stroke volume variation. During expiration, preload of the right ventricle is lowered and during inspiration it increases, contrary to mechanical ventilation. Apart from these dynamic indices, an endogenous fluid challenge, the passive leg raising test, has been proposed as a predictive test for fluid responsiveness [31] for intubated patients as well as patients breathing spontaneously.

\subsubsection{Measurement of dynamic indices}

Soubrier et al [32] evaluated PPV in unstable patients breathing spontaneously. Thirtytwo patients received a fluid challenge of $500 \mathrm{ml}$ (6\% hydroxyethyl starch). A PPV of $\geq 12 \%$ resulted in a sensitivity of $63 \%$ and a specificity of $92 \%$. The low sensitivity can be explained by insufficient changes in pleural pressure when breathing spontaneously, as has also been shown in mechanically-ventilated patients with low tidal volumes [11]. The high specificity, however, was a remarkable finding. This implicates that when a PPV $\geq 12 \%$ is present in a patient breathing spontaneously, a response to fluid is likely. These results, however, are in contrast to the findings of 
Heenen et al [33]. In 12 patients, breathing spontaneously through a face mask with oxygen, PPV had an area under the ROC curve of $0.29 \pm 0.17$ for prediction of fluid responsiveness. The use of PPV in spontaneously breathing patients therefore is still questionable [34].

Perner et al. [35] studied SVV, measured with the PiCCO system, in 30 patients with septic shock ventilated in the pressuresupport mode. A fluid challenge of 500 $\mathrm{ml}$ of colloid was given. Responders were defined as having an increase of $>10 \%$ in the cardiac index. SVV did not change significantly before and after the fluid challenge ( $13 \pm 5 \%$ vs. $16 \pm 6 \%, \mathrm{p}=0.26)$. Mean area under the ROC curve was 0.52 (95\% CI, 0.39-0.73). It was concluded that SVV does not predict the response to a fluid challenge in patients on pressure support. Similar results were found by Heenen et al [33] in mixed group of 9 critically ill patients on pressure support with an area under the ROC curve of $0.64 \pm 0.26$.

Magder et al [36] raised the hypothesis that right atrial pressure does not decrease during voluntary inspiration if the heart is not volume responsive. Inspiration and expiration cause a variable preload to the right ventricle depending on where the heart is operating on the Starling curve. This concept was tested in 33 patients after cardiopulmonary surgery. Twelve patients were breathing spontaneously and 21 were breathing in an assist mode. All patients received fluid loading in order to increase CVP more than $2 \mathrm{mmHg}$. In only 1 out of 14 patients with an absent respiratory response on right atrial pressure did cardiac output increase more than $250 \mathrm{ml} / \mathrm{min}$. In the group with a positive respiratory response on right atrial pressure (decrease in $\mathrm{CVP} \geq 1 \mathrm{mmHg}$ during inspiration), fluid loading resulted in an increase in cardiac output of more than $250 \mathrm{ml} / \mathrm{h}$ in 16 out of 19 patients. Comparable results were found in an additional study by Magder et al [38]. Heenen et al [34] studied this concept in 9 critically ill patients on pressure support and 12 patients breathing spontaneously. The predictive value to identify responders to fluid was poor, with an area under the ROC curve of $0.53 \pm 0.13$ (mean $\pm \mathrm{SD}$ ). No separate analysis was made for patients on pressure support or those breathing spontaneously.

\subsubsection{Passive leg raising test}

Raising the legs to 45 for 4 minutes results in a transient increase in venous return [37]. Using radiolabelled erythrocytes, it was shown that the infused volume of blood from the legs is approximately $150 \mathrm{ml}$ [38]. Besides raising the legs, the trunk of the patient can be positioned horizontally to maximize the effect of the endogenous volume challenge [39]. The amount of the endogenous fluid challenge will be vary between patients and strongly depends on vasomotor tone. In a hypovolaemic, vasoconstricted patient less volume will be recruited than in a vasodilated patient in septic shock. Theoretically the PLR test might be false negative in severely vasoconstricted patients. However, most of these clinical situations are straightforward, e.g. severe 
hypovolaemia.

According to the Starling principle, a PLR test increases CI immediately when the heart is on the steep portion of the curve. Various studies have shown that a PLR test is able to increase CI and that CI returns to baseline when lowering the legs [39]. Therefore the PLR test can be regarded as a completely reversible, endogenous volume challenge. The haemodynamic changes occur within seconds and are maximal approximately 1 minute after starting the manoeuvre [40].

Boulain et al [31] showed in fully sedated, mechanically-ventilated patients that changes in stroke volume induced by passive leg raising (PLR), and infusion of 300 $\mathrm{ml}$ gelatin were strongly correlated $(\mathrm{r}=0.89, \mathrm{p}<0.001)$. Monnet et al [40] found that the PLR predicted fluid responsiveness with a sensitivity of $97 \%$ and a specificity of $94 \%$ in 71 mechanically ventilated patients, of whom 31 had spontaneous breathing activity and/or arrhythmias. Lafanchere et al [18] conducted a similar study in 22 fully sedated and mechanically-ventilated, critically-ill patients. The PLR test had a sensitivity of $90 \%$ and a specificity of $83 \%$ to predict an increase in aortic blood flow of $15 \%$. Galas et al. [41] found a sensitivity of $95 \%$ and a specificity of $94 \%$ for the PLR to predict fluid responsiveness in 44 patients on controlled mechanical ventilation after cardiac surgery. Fourteen patients were included with an irregular heart rate.

Lamia et al [42] conducted a study in 14 patients on assisted mechanical ventilation and 10 patients breathing spontaneously. The PLR test had a sensitivity of $77 \%$ and a specificity of $100 \%$ for predicting fluid responsiveness. There was no difference between intubated and non-intubated patients. In this study transthoracic echocardiography was used to measure stroke volume. Other echocardiographic measures, such as E/Ea and left ventricular end-diastolic area, were not useful for predicting fluid responsiveness.

As shown by these studies, the PLR test can be used in ventilated patients and in patients breathing spontaneously, and is independent of cardiac arrhythmias. However, the PLR test has several limitations. This method requires the continuous measurement of changes in cardiac output. Transoesophageal Doppler was used in the study of Monnet et al [40] Lamia et al [42] used transthoracic echocardiography. Today there are numerous methods for rapid and valid measurement of cardiac output [43]. Changes in blood pressure are not sufficient to evaluate the effect of a PLR test $[40,42]$. In some patients the PLR test leads to considerable discomfort or is not possible, e.g. in trauma patients.

\subsection{Clinical algorithm}

Nowadays it is possible to predict fluid responsiveness in the majority of intensive care patients. However, different methods have to be used in different clinical situ- 
ations. The method of choice is mainly directed by the limitations of a method and skills of the doctor. A clinical flow chart for choosing a method is shown in Figure 3.

After diagnosing inadequate organ perfusion, the first step is to determine the patients heart rhythm. An irregular heart rhythm excludes the use of dynamic indices such as SPV, PPV and SVV. The use of echocardiography to assess the collapsibility of the ICV or SCV has not been validated in cases of irregular heart rhythm. A PLR test is the most valid option.

If the patient is on controlled mechanical ventilation, has a regular heart rhythm and the tidal volume is $8 \mathrm{ml} / \mathrm{kg}$, we advise the use of SVV or PPV. These indices are easy to monitor and can be measured continuously. If SVV or PPV is $>12 \%$, we advise fluid administration if clinical or biochemical signs of tissue hypoperfusion are present. Alternative measurements in this patient category are the ICV distensibility or SCV collapsibility indices. If tidal volume is $<8 \mathrm{ml} / \mathrm{kg}$, sensitivity for prediction of fluid responsiveness using SVV and PVV rapidly declines. However, as Huang et al [21] have shown in severe ARDS patients, specificity may still be high. De Backer et al [11] found a specificity of only $65 \%$ for PPV in a mixed group of intensive care patients using a tidal volume $<8 \mathrm{ml} / \mathrm{kg}$. In our opinion, the use of SVV or $\mathrm{PPV}$ in case of a tidal volume $<8 \mathrm{ml} / \mathrm{kg}$ needs more validation to be clinically useful. Therefore we advise a PLR test in case of a tidal volume $<8 \mathrm{ml} / \mathrm{kg}$. If a PLR test cannot be performed, a traditional fluid challenge must be done with a small, rapid bolus, e.g. $250 \mathrm{ml}$, with monitoring of CO. If cardiac index does not increase $>15 \%$, fluid loading should be stopped.

For patients on mechanical ventilation with spontaneous activity the only method validated in the literature is the PLR test. Further research is needed on dynamic indices in these patients. A fluid challenge with $\mathrm{CO}$ measurement should be performed if PLR is not possible.

In patients breathing spontaneously more evidence is needed to support the use of SVV or PPV. Although Soubrier et al [32] showed that specificity still may be high, SVV or PPV can not yet be advised to predict fluid responsiveness in these patients. The same is true for the measurement of the inspiratory drop in CVP proposed by Magder et al $[36,44]$. Therefore, in this situation we advise a PLR test. Otherwise, a fluid challenge with CO measurement is indicated if a PLR test is not possible.

In conclusion, prediction of fluid responsiveness is possible in most critically ill patients and should be implemented in routine clinical practice. The suggested algorithm may prevent inappropriate fluid boluses in most critically ill patients. Future studies should address the question if a fluid management strategy based on prediction of fluid responsiveness results in an improvement in clinical outcome. 


\section{References}

[1] D. Schuller, J. P. Mitchell, F. S. Calandrino, and D. P. Schuster, "Fluid balance during pulmonary edema. is fluid gain a marker or a cause of poor outcome?" Chest, vol. 100, no. 4, pp. 1068-75, 1991.

[2] S. G. Sakka, M. Klein, K. Reinhart, and A. Meier-Hellmann, "Prognostic value of extravascular lung water in critically ill patients," Chest, vol. 122, no. 6, pp. 2080-6, 2002.

[3] H. P. Wiedemann, A. P. Wheeler, G. R. Bernard, B. T. Thompson, D. Hayden, B. deBoisblanc, J. Connors, A. F., R. D. Hite, and A. L. Harabin, "Comparison of two fluid-management strategies in acute lung injury," The New England journal of medicine, vol. 354, no. 24, pp. 2564-75, 2006.

[4] J. Boldt, "[volume replacement in critically ill intensive-care patients. no classic review]," Anaesthesist, vol. 47, no. 9, pp. 778-85, 1998.

[5] D. Osman, C. Ridel, P. Ray, X. Monnet, N. Anguel, C. Richard, and J. L. Teboul, "Cardiac filling pressures are not appropriate to predict hemodynamic response to volume challenge," Crit Care Med, vol. 35, no. 1, pp. 64-8, 2007.

[6] P. E. Marik, M. Baram, and B. Vahid, "Does central venous pressure predict fluid responsiveness? a systematic review of the literature and the tale of seven mares," Chest, vol. 134, no. 1, pp. 172-8, 2008.

[7] B. Tavernier, O. Makhotine, G. Lebuffe, J. Dupont, and P. Scherpereel, "Systolic pressure variation as a guide to fluid therapy in patients with sepsis-induced hypotension," Anesthesiology, vol. 89, no. 6, pp. 1313-21, 1998.

[8] F. Michard, S. Boussat, D. Chemla, N. Anguel, A. Mercat, Y. Lecarpentier, C. Richard, M. R. Pinsky, and J. L. Teboul, "Relation between respiratory changes in arterial pulse pressure and fluid responsiveness in septic patients with acute circulatory failure," Am J Respir Crit Care Med, vol. 162, no. 1, pp. 134-8, 2000.

[9] A. Kramer, D. Zygun, H. Hawes, P. Easton, and A. Ferland, "Pulse pressure variation predicts fluid responsiveness following coronary artery bypass surgery," Chest, vol. 126, no. 5, pp. 1563-8, 2004.

[10] D. A. Reuter, A. Kirchner, T. W. Felbinger, F. C. Weis, E. Kilger, P. Lamm, and A. E. Goetz, "Usefulness of left ventricular stroke volume variation to assess fluid responsiveness in patients with reduced cardiac function," Critical care medicine, vol. 31, no. 5, pp. 1399-404, 2003. 
[11] D. De Backer, S. Heenen, M. Piagnerelli, M. Koch, and J. L. Vincent, "Pulse pressure variations to predict fluid responsiveness: influence of tidal volume," Intensive Care Med, vol. 31, no. 4, pp. 517-23, 2005.

[12] C. K. Hofer, S. M. Muller, L. Furrer, R. Klaghofer, M. Genoni, and A. Zollinger, "Stroke volume and pulse pressure variation for prediction of fluid responsiveness in patients undergoing off-pump coronary artery bypass grafting," Chest, vol. 128 , no. 2, pp. 848-54, 2005.

[13] S. Preisman, S. Kogan, H. Berkenstadt, and A. Perel, "Predicting fluid responsiveness in patients undergoing cardiac surgery: functional haemodynamic parameters including the respiratory systolic variation test and static preload indicators," Br J Anaesth, vol. 95, no. 6, pp. 746-55, 2005.

[14] X. Monnet, M. Rienzo, D. Osman, N. Anguel, C. Richard, M. R. Pinsky, and J. L. Teboul, "Esophageal doppler monitoring predicts fluid responsiveness in critically ill ventilated patients," Intensive care medicine, vol. 31, no. 9, pp. 1195-201, 2005.

[15] H. Solus-Biguenet, M. Fleyfel, B. Tavernier, E. Kipnis, J. Onimus, E. Robin, G. Lebuffe, C. Decoene, F. R. Pruvot, and B. Vallet, "Non-invasive prediction of fluid responsiveness during major hepatic surgery," Br J Anaesth, vol. 97, no. 6 , pp. 808-16, 2006.

[16] C. Charron, C. Fessenmeyer, C. Cosson, J. X. Mazoit, J. L. Hebert, D. Benhamou, and A. R. Edouard, "The influence of tidal volume on the dynamic variables of fluid responsiveness in critically ill patients," Anesth Analg, vol. 102, no. 5, pp. 1511-7, 2006.

[17] G. Natalini, A. Rosano, M. Taranto, B. Faggian, E. Vittorielli, and A. Bernardini, "Arterial versus plethysmographic dynamic indices to test responsiveness for testing fluid administration in hypotensive patients: a clinical trial," Anesthesia and analgesia, vol. 103, no. 6, pp. 1478-84, 2006.

[18] A. Lafanechere, F. Pene, C. Goulenok, A. Delahaye, V. Mallet, G. Choukroun, J. D. Chiche, J. P. Mira, and A. Cariou, "Changes in aortic blood flow induced by passive leg raising predict fluid responsiveness in critically ill patients," Critical care, vol. 10, no. 5, p. R132, 2006.

[19] M. Feissel, J. L. Teboul, P. Merlani, J. Badie, J. P. Faller, and K. Bendjelid, "Plethysmographic dynamic indices predict fluid responsiveness in septic ventilated patients," Intensive care medicine, vol. 33, no. 6, pp. 993-9, 2007. 
[20] M. Cannesson, Y. Attof, P. Rosamel, O. Desebbe, P. Joseph, O. Metton, O. Bastien, and J. J. Lehot, "Respiratory variations in pulse oximetry plethysmographic waveform amplitude to predict fluid responsiveness in the operating room," Anesthesiology, vol. 106, no. 6, pp. 1105-11, 2007.

[21] C. C. Huang, J. Y. Fu, H. C. Hu, K. C. Kao, N. H. Chen, M. J. Hsieh, and Y. H. Tsai, "Prediction of fluid responsiveness in acute respiratory distress syndrome patients ventilated with low tidal volume and high positive end-expiratory pressure," Crit Care Med, vol. 36, no. 10, pp. 2810-6, 2008.

[22] R. Brower, R. A. Wise, C. Hassapoyannes, B. Bromberger-Barnea, and S. Permutt, "Effect of lung inflation on lung blood volume and pulmonary venous flow," Journal of applied physiology, vol. 58, no. 3, pp. 954-63, 1985.

[23] S. Preisman, E. DiSegni, Z. Vered, and A. Perel, "Left ventricular preload and function during graded haemorrhage and retranfusion in pigs: analysis of arterial pressure waveform and correlation with echocardiography," British journal of anaesthesia, vol. 88, no. 5, pp. 716-8, 2002.

[24] R. Pizov, Y. Ya'ari, and A. Perel, "The arterial pressure waveform during acute ventricular failure and synchronized external chest compression," Anesthesia and analgesia, vol. 68, no. 2, pp. 150-6, 1989.

[25] A. Perel, R. Pizov, and S. Cotev, "Systolic blood pressure variation is a sensitive indicator of hypovolemia in ventilated dogs subjected to graded hemorrhage," Anesthesiology, vol. 67, no. 4, pp. 498-502, 1987.

[26] F. Michard, A. Descorps-Declere, and M. R. Lopes, "Using pulse pressure variation in patients with acute respiratory distress syndrome," Critical care medicine, vol. 36, no. 10, pp. 2946-8, 2008.

[27] H. K. Kim and M. R. Pinsky, "Effect of tidal volume, sampling duration, and cardiac contractility on pulse pressure and stroke volume variation during positive-pressure ventilation," Crit Care Med, vol. 36, no. 10, pp. 2858-62, 2008.

[28] M. Feissel, F. Michard, J. P. Faller, and J. L. Teboul, "The respiratory variation in inferior vena cava diameter as a guide to fluid therapy," Intensive Care Med, vol. 30, no. 9, pp. 1834-7, 2004.

[29] C. Barbier, Y. Loubieres, C. Schmit, J. Hayon, J. L. Ricome, F. Jardin, and A. Vieillard-Baron, "Respiratory changes in inferior vena cava diameter are helpful in predicting fluid responsiveness in ventilated septic patients," Intensive Care Med, vol. 30, no. 9, pp. 1740-6, 2004. 
[30] A. Vieillard-Baron, K. Chergui, A. Rabiller, O. Peyrouset, B. Page, A. Beauchet, and F. Jardin, "Superior vena caval collapsibility as a gauge of volume status in ventilated septic patients," Intensive Care Med, vol. 30, no. 9, pp. 1734-9, 2004.

[31] T. Boulain, J. M. Achard, J. L. Teboul, C. Richard, D. Perrotin, and G. Ginies, "Changes in bp induced by passive leg raising predict response to fluid loading in critically ill patients," Chest, vol. 121, no. 4, pp. 1245-52, 2002.

[32] S. Soubrier, F. Saulnier, H. Hubert, P. Delour, H. Lenci, T. Onimus, S. Nseir, and A. Durocher, "Can dynamic indicators help the prediction of fluid responsiveness in spontaneously breathing critically ill patients?" Intensive Care Med, vol. 33, no. 7, pp. 1117-24, 2007.

[33] S. Heenen, D. De Backer, and J. L. Vincent, "How can the response to volume expansion in patients with spontaneous respiratory movements be predicted?" Crit Care, vol. 10, no. 4, p. R102, 2006.

[34] D. De Backer and M. R. Pinsky, "Can one predict fluid responsiveness in spontaneously breathing patients?" Intensive care medicine, vol. 33, no. 7, pp. 11113, 2007.

[35] A. Perner and T. Faber, "Stroke volume variation does not predict fluid responsiveness in patients with septic shock on pressure support ventilation," Acta anaesthesiologica Scandinavica, vol. 50, no. 9, pp. 1068-73, 2006.

[36] S. Magder, G. Georgiadis, and T. Cheong, "Respiratory variations in right atrial pressure predict the response to fluid challenge," J Crit Care, vol. 7, no. 2, pp. 76-85, 1992.

[37] M. Thomas and J. Shillingford, "The circulatory response to a standard postural change in ischaemic heart disease," British heart journal, vol. 27, pp. 17-27, 1965.

[38] D. L. Rutlen, F. J. Wackers, and B. L. Zaret, "Radionuclide assessment of peripheral intravascular capacity: a technique to measure intravascular volume changes in the capacitance circulation in man," Circulation, vol. 64, no. 1, pp. 146-52, 1981.

[39] X. Monnet and J. L. Teboul, "Passive leg raising," Intensive Care Med, vol. 34, no. 4, pp. 659-63, 2008.

[40] X. Monnet, M. Rienzo, D. Osman, N. Anguel, C. Richard, M. R. Pinsky, and J. L. Teboul, "Passive leg raising predicts fluid responsiveness in the critically ill," Crit Care Med, vol. 34, no. 5, pp. 1402-7, 2006. 
[41] F. Galas, L. Hajjar, T. Polastri, T. Faustino, W. Leao, L. Sampaio, and J. Auler, "Passive leg raising predicts fluid responsiveness after cardiac surgery," Crit Care, vol. 12, no. Suppl 2, p. 1, 2008.

[42] B. Lamia, A. Ochagavia, X. Monnet, D. Chemla, C. Richard, and J. L. Teboul, "Echocardiographic prediction of volume responsiveness in critically ill patients with spontaneously breathing activity," Intensive Care Med, vol. 33, no. 7, pp. 1125-32, 2007.

[43] C. K. Hofer, M. T. Ganter, and A. Zollinger, "What technique should i use to measure cardiac output?" Current opinion in critical care, vol. 13, no. 3, pp. 308-17, 2007.

[44] S. Magder and D. Lagonidis, "Effectiveness of albumin versus normal saline as a test of volume responsiveness in post-cardiac surgery patients," Journal of critical care, vol. 14, no. 4, pp. 164-71, 1999. 


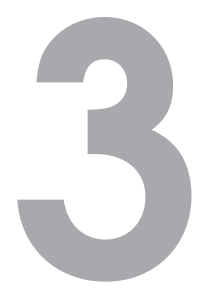

\title{
Dynamic indices do not predict volume responsiveness in routine clinical practice
}

\author{
B. Lansdorp, J. Lemson, M.J.A.M van Putten, A. de Keijzer, J. G. van der Hoeven \\ and P. Pickkers \\ British Journal of Anaesthesia 2012 Mar;108(3):395-401
}

Background. Dynamic indices, including pulse pressure, systolic pressure, and stroke volume variation (PPV, SPV, and SVV), are accurate predictors of fluid responsiveness under strict conditions, for example, controlled mechanical ventilation using conventional tidal volumes (TVs) in the absence of cardiac arrhythmias. However, in routine clinical practice, these prerequisites are not always met. We evaluated the effect of regularly used ventilator settings, different calculation methods, and the presence of cardiac arrhythmias on the ability of dynamic indices to predict fluid responsiveness in sedated, mechanically ventilated patients.

Methods. We prospectively evaluated 47 fluid challenges in 29 cardiac surgery patients. Patients were divided into different groups based on TV. Dynamic indices were calculated in various ways: calculation over 30s, breath-by-breath (with and without excluding arrhythmias), and with correction for TV.

Results. The predictive value was optimal in the group ventilated with TVs $>7 \mathrm{ml} \cdot \mathrm{kg}^{-1}$ with correction for TV, calculated breath-by-breath, and with exclusion of arrhythmias [area under the curve (AUC) = 0.95, 0.93, and 0.90 for PPV, SPV, and SVV, respectively]. Including patients ventilated with lower TVs decreased the predictive value of all dynamic indices, while calculating dynamic indices over $30 \mathrm{~s}$ and not excluding cardiac arrhythmias further reduced the AUC to 0.51, 0.63, and 0.51 for PPV, SPV, and SVV, respectively. 
Conclusions. PPV, SPV, and SVV are the only reliable predictors of fluid responsiveness under strict conditions. In routine clinical practice, factors including low TV, cardiac arrhythmias, and the calculation method can substantially reduce their predictive value. 


\subsection{Introduction}

Estimating the intravascular volume status of the intensive care unit (ICU) patients remains a clinical challenge. While hypovolaemia can result in inadequate organ perfusion and organ dysfunction, inappropriate fluid administration can result in pulmonary and interstitial oedema and contribute to further tissue injury, organ dysfunction, and eventually death [1-4]. Therefore, reliable predictors of fluid responsiveness are highly relevant.

Various dynamic indices, based on cardiopulmonary interactions in ventilated patients, have been shown to accurately predict fluid responsiveness [5-9]. These dynamic indices are induced by mechanical ventilation when the heart operates on the steep portion of the FrankStarling curve $[10,11]$ and include pulse pressure variation (PPV), systolic pressure variation (SPV), and stroke volume variation (SVV).

Although several studies [5-9] have shown excellent accuracy for these dynamic indices, most of the study populations were strictly controlled, including controlled mechanical ventilation with no spontaneous breathing, tidal volumes (TVs) $>7$ $\mathrm{ml} \cdot \mathrm{kg}^{-1}$, breath-by-breath calculation, and no cardiac arrhythmias. However, recent research including more than 12000 consecutive patients undergoing surgery [12] showed that only $39 \%$ of patients met the criteria for monitoring fluid responsiveness, with pulse pressure measured invasively or non-invasively [13]. This is in part because patients are ventilated with low TVs of 6-8 $\mathrm{ml} \cdot \mathrm{kg}^{-1}$ [14-16], calculation of dynamic indices is not performed on a breath-by-breath basis, and patients often show spontaneous breathing activity. In addition, cardiac arrhythmias can occur on an irregular basis. Nevertheless, dynamic indices are often used in routine clinical practice while the exact effects of these non-ideal circumstances on the predictive value of dynamic indices are unclear. This emphasizes the need for quantification of their influence on dynamic indices in the general cardiac ICU patient.

The aim of the present study was to evaluate the effects of regularly used ventilator settings, cardiac arrhythmias, and different calculation methods on the predictive value of dynamic indices on fluid responsiveness in sedated, mechanically ventilated patients after cardiac surgery.

\subsection{Methods}

\subsubsection{Patients}

Thirty patients on controlled mechanical ventilation after isolated coronary artery bypass surgery were prospectively studied from the time of admission to the ICU. Because of the observational and non-invasive character of this study, the local medical ethics committee waived the need for informed consent. Fluid challenges were administered by the attending physician based upon the presence of at least one clin- 


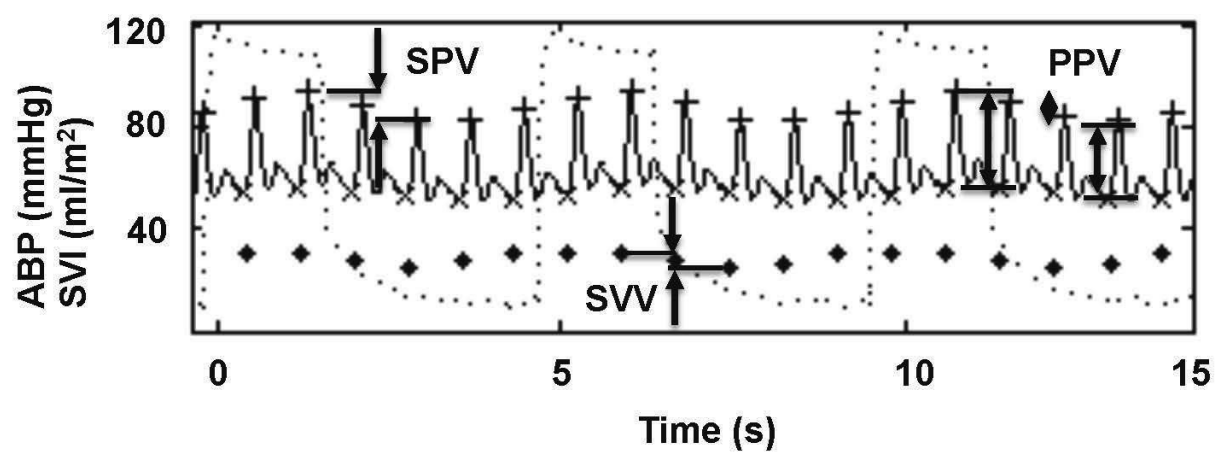

Figure 1: Example of processed data. Ventilator inspiratory pressure (dotted line), arterial pressure (ABP, with marked systolic and diastolic pressure $(\mathrm{mmHg}))$, stroke volume index $\left(S V I\right.$, dots $\left.\left(m l \cdot m^{-2}\right)\right)$, with illustrated PPV, SPV, and SVV.

ical sign of inadequate tissue perfusion [low mean arterial pressure (MAP), low urine production, cold extremities, elevated lactate level, or low central venous oxygen saturation]. Patients were excluded if they did not receive a single fluid challenge, showed spontaneous respiratory efforts, or had an intra-aortic balloon pump.

All patients arrived on the ICU with a central venous catheter in the internal jugular position and a radial arterial catheter $(20 \mathrm{G})$. Mechanical ventilation was performed with the Servo 300 (Macquet, Rasstat, Germany). Vasoactive medication was administered according to standard clinical protocols.

\subsubsection{Haemodynamic monitoring}

Arterial pressure (AP), central venous pressure (CVP), and EKG were monitored (Merlin M1046A monitor, Hewlett Packard, Palo Alto, CA, USA) and inspiratory pressure (including Ppeak, Pplat, and PEEP) and inspiratory flow were collected on a computer using the serial port of the Servo 300 ventilator. Both haemodynamic and ventilator parameters were continuously recorded on a laptop computer and stored on a hard disk with a sample rate of $200 \mathrm{~Hz}$ by an A/D converter (NI USB-6211, National Instrument, Austin, TX, USA). Afterwards, cardiac output (CO) and stroke volume (SV) were derived offline with the pulse contour method using the same algorithm incorporated in the BMEYE Nexfin Monitor (BMEYE, Amsterdam, The Netherlands) $[17,18]$.

From the recorded AP and SV, the PPV, SPV, and SVV were calculated offline. Dynamic indices are defined by the relative difference in maximal and minimal pulse pressure (PPmax/PPmin), systolic pressure (SPmax/SPmin), and SV (SV- 


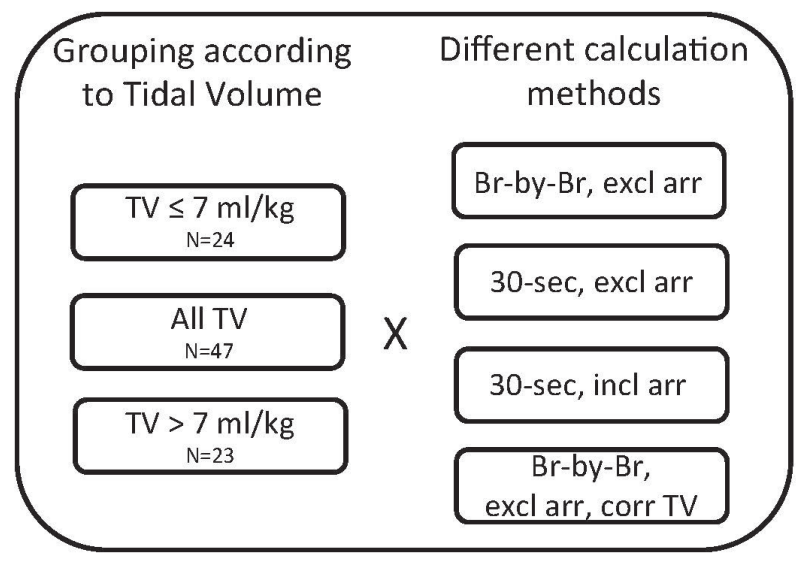

Figure 2: Summary of different analysis groups based on tidal volume and calculation method. Arr, arrhythmia; Br, breath; TV, tidal volume.

max/SVmin) for PPV, SPV, and SVV, respectively, according to:

$$
100 \cdot \frac{Q_{\max }-Q_{\min }}{\left(Q_{\max }+Q_{\min }\right) / 2}
$$

with Q=PP, SP, SV for PPV, SPV, and SVV, respectively (Figure 1) [19]. All indices were automatically detected by an algo- Q3 rithm written in Matlab (Matlab R2009b, MathWorks Inc., Natick, MA, USA). The maxima and minima calculated by the software were visually inspected for errors.

\subsubsection{Design}

All patients were mechanically ventilated using the pressure-regulated volume control mode. Recording of physiological data started immediately after arrival on the ICU and included every fluid challenge administered. The volume challenge consisted of the infusion of $250 \mathrm{ml}$ of 130/0.4 6\% HES solution (Voluven, Frensenius Kabi, s-Hertogenbosch, The Netherlands).

Patients were identified as a responder to fluid challenge if their SV increased by $>10 \%$ [20, 21]. Baseline measurement was performed 3 min before start of the fluid challenge, and response was measured within 3 min after the infusion was completed.

To investigate the influence of TV, patients were analysed in three different groups according to their TV. One analysis included all patients (all TVs). Subsequently, we analysed patients with TV higher and lower than $7 \mathrm{ml} \cdot \mathrm{kg}^{-1}$, a threshold used ear- 
lier.5 To investigate the influence of the calculation method and arrhythmias, for all the three TV groups, the dynamic indices were calculated in four different ways. First, dynamic indices were calculated breath-by-breath and averaged over a selected period of 5 breaths, excluding cardiac arrhythmias.7 Secondly, the indices were calculated over a period of 30s (excluding cardiac arrhythmias) by using the mean of four maximal and four minimal PP, SP, and SV values to calculate the PPV, SPV, and SVV, respectively. The same method for the calculation over 30s was used again for the third analysis only now without excluding cardiac arrhythmias occurring within the 30s around baseline. Finally, dynamic indices were calculated breath-by-breath and divided by the delivered TV to correct for the use of different TVs [22], excluding cardiac arrhythmias. This resulted in a total of 12 different analyses per dynamic index (Figure 2).

\subsubsection{Statistical analysis}

Changes in haemodynamic and respiratory parameters due to volume expansion were analysed using the Wilcoxon rank test. Differences in baseline and response to fluid challenge between responders and non-responders were assessed using the MannWhitney U-test. Linear correlations were tested using the Spearman rank method. Receiver operator characteristic (ROC) curves were constructed for the dynamic indices to evaluate the predictive value together with the sensitivity, specificity, and positive and negative likelihood ratio. P-values of $<0.05$ were considered statistically significant. Because of the small number of patients, no formal statistical tests on the ROC curves from the different subgroups were performed. Statistical analysis was performed using SPSS 18 for windows (SPSS Inc., Chicago, IL, USA).

Table 1: Patient characteristics and ventilatory parameters.

\begin{tabular}{||l|c|c||}
\hline & Median & IQR \\
\hline Patient (male) $(\#)$ & $29(25)$ & \\
Age $(y r)$ & 67 & $61-71$ \\
Weight $(\mathrm{kg})$ & 91 & $74-99$ \\
Respiratory rate $(\mathrm{bpm})$ & 12.6 & $12.2-14.3$ \\
Inspiratory pressure $\left(\mathrm{cmH}_{2} \mathrm{O}\right)$ & 16.6 & $15.3-18.8$ \\
PEEP $\left(\mathrm{cmH}_{2} \mathrm{O}\right)$ & 5.3 & $4.6-6.9$ \\
Tidal volume $\left(\mathrm{ml} \cdot \mathrm{kg}_{1} I \mathrm{IBW}\right)$ & 7.0 & $4.0-10.0$ \\
Ventilatory mode & Pressure-regulated volume control \\
\hline Fluid challenges $(\#)$ & 47 & \\
Infusion volume $(\mathrm{ml})$ & 250 & \\
Infusion duration $(\mathrm{min})$ & 7 & $4-10$ \\
\hline
\end{tabular}




\subsection{Results}

One patient was excluded because of spontaneous breathing activity. The remaining study population consisted of 29 patients receiving a total of 47 fluid challenges. Patient characteristics, relevant ventilator parameters, and data concerning fluid challenges are presented in Table 1. Seven fluid challenges (15\%) resulted in an increase in $\mathrm{SV}>10 \%$ (responders).

Baseline haemodynamic parameters were not significantly different between responders and non-responders except for a higher MAP in non-responders. Figure 3 shows the haemodynamic variables at baseline and after volume expansion in all responders and non-responders. MAP and CVP increased significantly in response to fluid administration for both responders and non-responders, whereas HR decreased significantly in both groups. The change in HR and SVI was significantly higher in responders compared with non-responders.

In general, baseline measurements of PPV, SPV, and SVV were significantly different between responders and nonresponders, and also the decrease in these parameters as a result of fluid challenge (Figure 4). Figure 5 illustrates that including patients ventilated with lower TVs decreases the predictive value of all three dynamic indices. For example, for PPV (Figure 5A), adding patients ventilated with all TV $(n=47)$ to the patient group ventilated with TV $>7 \mathrm{ml} \cdot \mathrm{kg}^{-1}(\mathrm{n}=23)$ decreased the area under the curve (AUC) from 0.92 to 0.80 , and further to 0.69 in patients ventilated with $\mathrm{TV}<7 \mathrm{ml} \cdot \mathrm{kg}^{-1}(\mathrm{n}=24)$. When changing the calculation method from breath-bybreath analysis to calculation over 30s without excluding cardiac arrhythmias, the AUC for PPV in patients ventilated with TV $>7 \mathrm{ml} \cdot \mathrm{kg}^{-1}$ decreased from 0.92 to 0.79. On the other hand, correction of the PPV for TV improved the AUC from 0.92 to 0.95 with a sensitivity and specificity of $100 \%$ and $93 \%$, respectively. This particular group also showed the highest values for SPV (AUC=0.93, Figure 5B) and SVV (AUC $=0.90$, Figure $5 \mathrm{C}$ ). SPV was least influenced by the varying circumstances. The ROC curves in Figure 5D show the decrease in predictive value of PPV in four characteristic groups.

\subsection{Discussion}

The present study confirms previous reports $[5,6,9]$ that show that the ability of dynamic indices to predict volume responsiveness is very good under strictly regulated conditions. In addition, the main finding of our study is that factors present in routine clinical practice, such as the use of lower TVs, cardiac arrhythmias, and the method of calculation, negatively influence the predictive value of dynamic indices. Unrestricted use of dynamic indices is therefore not without pitfalls since almost half of the TVs applied nowadays are $7 \mathrm{ml} \cdot \mathrm{kg}^{-1}$ [23]. Dynamic indices are mostly calcu- 

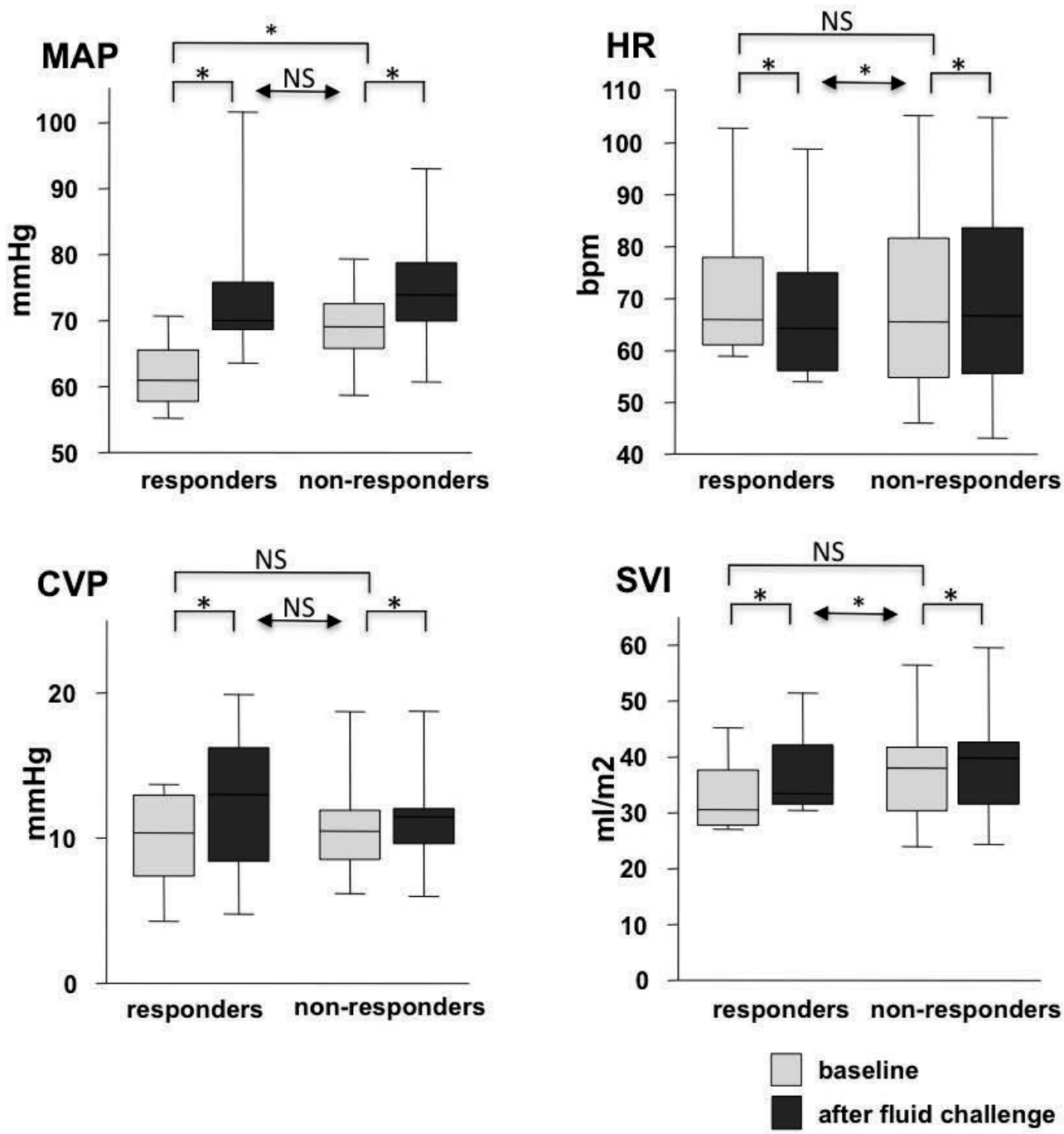

Figure 3: Comparison of main haemodynamic variables [MAP (A), HR (B), CVP $(C)$, and $S V I(D)]$ at baseline and after volume expansion in responders and non-responders. The difference between baseline and after fluid loading (left and right significance mark), difference in baseline between responder and non-responder (upper significance mark) and difference in change of the parameter due to volume expansion between responders and non-responders (lower significance mark) is shown. $* P<0.05 ; N S$, not significant. 

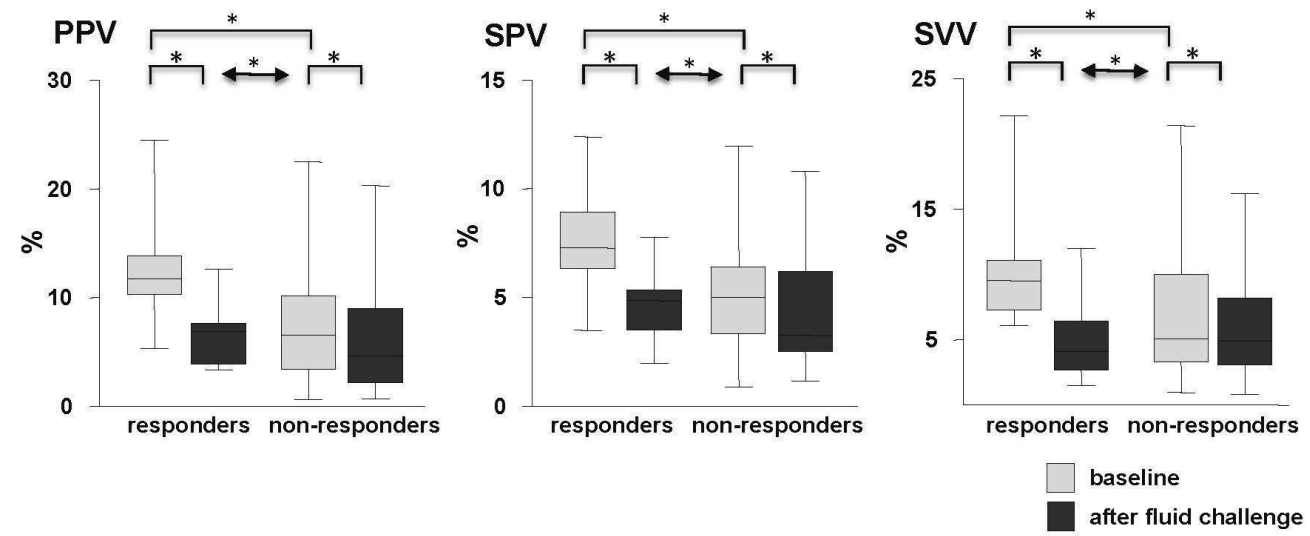

Figure 4: Comparison of dynamic indices at baseline and after volume expansion in responders and non-responders (breath-by-breath analysis in group with all tidal volumes, cardiac arrhythmias excluded). Significance is indicated $(* P<0.05)$ for the difference between baseline and after fluid loading (left and right significance mark), difference in baseline between responder and non-responder (upper significance mark), and difference in change of the parameter because of the volume expansion between responders and non-responders (lower significance mark).

lated automatically using various haemodynamic monitors that lack respiratory data and therefore calculate dynamic indices over a period of 30s. This also increases the risk of including cardiac arrhythmias into the calculation period because an unnecessary long time interval is analysed. Therefore, in routine clinical practice, liberal use of dynamic indices to support the clinical decisions to administer fluid results in a false sense of security.

\subsubsection{Influence of TV}

The effect of TV on the absolute value of dynamic indices has been acknowledged earlier [24-26], but to what extent TV influences the predictive value of the dynamic indices is sparsely studied. In a meta-analysis [5], most of the studies included also used a TV of 8-10 $\mathrm{ml} \cdot \mathrm{kg}^{-1}$, and they suggest this range when using dynamic indices to predict fluid responsiveness. In one study [27], it was shown that PPV is only reliable when TV $>8 \mathrm{ml} \cdot \mathrm{kg}^{-1}$ (difference in sensitivity and specificity of $22 \%$ and $24 \%$, respectively, in the high vs low TV group). This is most likely caused by a non-linear compliance of the chest wall resulting in less variation in intrathoracic pressure with decreased TVs. However, since in clinical practice, it is preferred to ventilate with lower TV than traditionally used [14-16, 28], we evaluated the use of dynamic indices using TV within this lower range. The influence of TV on the 

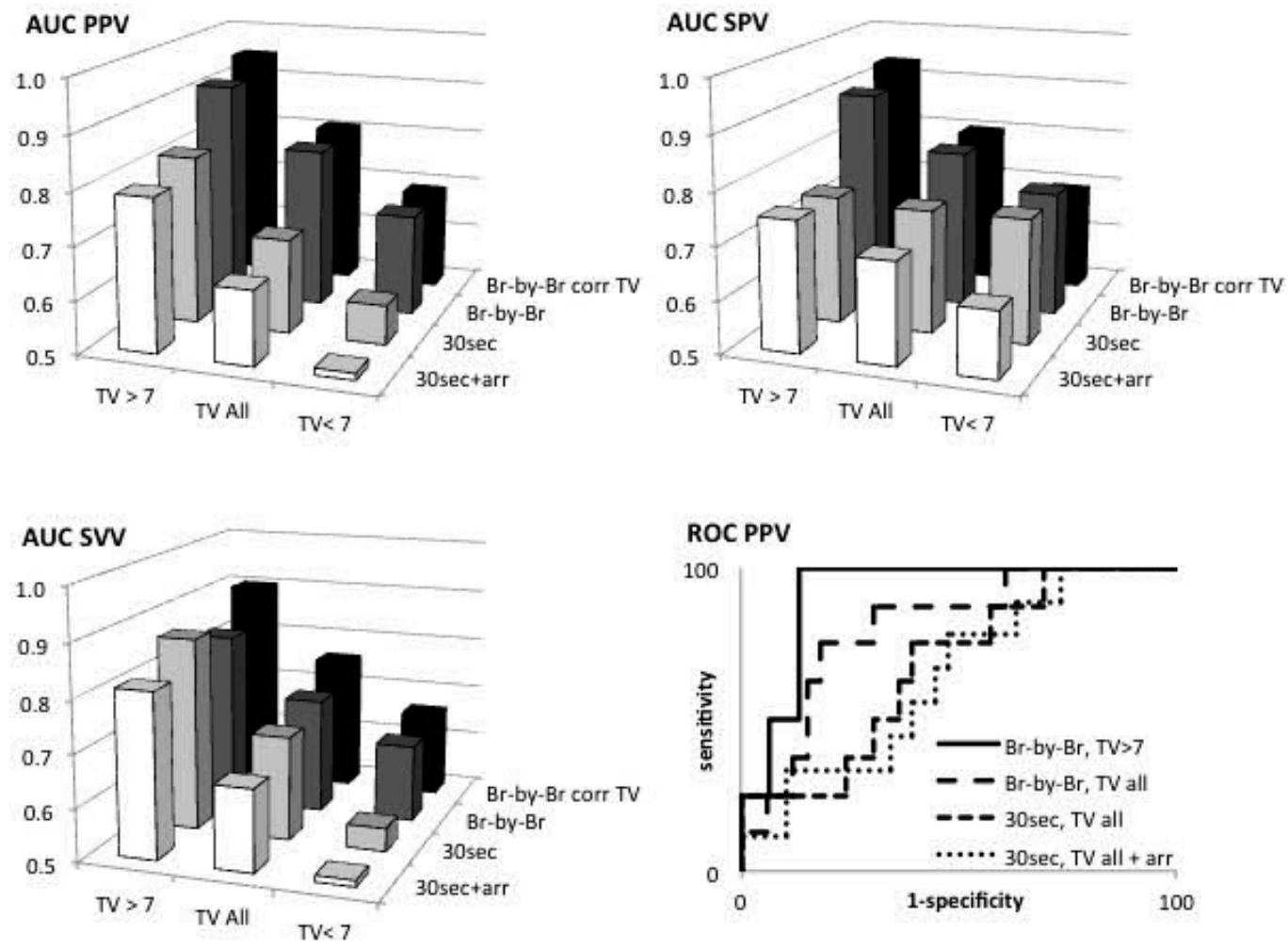

Figure 5: ROC curves and AUC of dynamic indices for different groups of tidal volume (n=23, 47, and 24, for the groups with $T V>7 \mathrm{ml} \cdot \mathrm{kg}^{-1}$, TV all, and $T V<7 \mathrm{ml} \cdot \mathrm{kg}^{-1}$, respectively). (AC) The areas under the ROC curve for PPV, SPV, and SVV for all different groups. (D) The four ROC curves for characteristic groups. 
absolute value of dynamic indices and their predictive value suggest that dynamic indices should be corrected for the applied TV. Indeed, when PPV is divided by the $\mathrm{TV}$, the predictive value of the PPV improves [22, 29]. In addition, our results show an improved predictive value, not only for PPV, but also for SPV and SVV, after adjustment for TV.

\subsubsection{Influence of different calculation methods}

By definition, dynamic indices such as SPV, PPV and SVV represent variation of the systolic pressure, pulse pressure, and SV, respectively, over a breath. Importantly, increasing the number of breaths over which the dynamic indices are calculated can increase the calculated values, because larger and smaller pulse pressures and SVs are more likely to occur during a longer observation period, resulting in a more pronounced variation in the dynamic indices [24]. However, clinical devices such as the Philips IntelliVue Patient Monitor (Philips Medical Systems, Eindhoven, The Netherlands), the PiCCO2 (Pulsion Medical Systems, Muenchen, Germany), and the Drager infinity (Drager Medical, Lubech, Germany) use software that samples a defined time interval without identifying the number of breaths. During low respiratory rates, this leads to significant variation in PPV, which has no physiological background and should therefore be discarded. On the other hand, in other cases, the time window of 30 s leads to an unnecessary high number of breaths, which increases the chance to include cardiac arrhythmias such as premature ventricular contractions. This negatively influences the predictive value of dynamic indices. The results from the present study confirm this hypothesis, as the most pronounced decrease in predictive value of the PPV was caused by a change in calculation from breath-by-breath to a 30s period.

\subsubsection{Influence of cardiac arrhythmias}

Dynamic indices reflect the influence of mechanical ventilation on cardiac preload. However, this is not the case during cardiac arrhythmias like atrial fibrillation or frequent premature ventricular contractions. Therefore, these cardiac arrhythmias should be excluded from calculations. Of importance, neglecting or interpolating the premature ventricular contraction is not advisable, since it is not known whether the neglected beat would represent a larger or smaller pulse pressure and the heart beat directly after a PVC occurs after a compensatory pause, resulting in a higher pulse pressure. For this reason, not only the specific beat, but the whole respiratory cycle should be excluded from the dynamic indices calculation. This is supported by the results of the present study. The extent to which cardiac arrhythmias influence the predictive value of dynamic indices of course depends on their incidence. In cardiac patients, the incidence of arrhythmias and thereby their influence might be higher than in other patient populations. 
A technique that is less dependent on changing clinical conditions is the passive leg raising (PLR) test. Non-invasive CO monitoring during the PLR test predicts volume responsiveness with high accuracy in a broad population of ICU patients [30,31], even in patients with spontaneous breathing activity [32]. Other alternatives independent from the TV include assessment of volume responsiveness during respiratory manoeuvres like the Valsalva manoeuvre $[33,34]$, end-expiratory occlusion [35], or the respiratory systolic variation test that uses three mechanical breaths with gradually increasing airway pressures [36]. A disadvantage that all these alternatives have is that they are progressively labour intensive, which reduces their applicability in routine practice.

\subsubsection{Limitations}

Changes in $\mathrm{CO}$ were monitored using the pulse contour method. Although previous validation studies show excellent accuracy of this technique [17, 18], and only changes in SVI were used (and not the absolute value), this method might be less reliable compared with more invasive methods. In contrast to previous studies, 5 we found a relatively low number of responders (15\%). Although other studies also used this amount of infused volume with comparable or even higher thresholds to identify volume responders [36,37], this might be related to the relatively low volume infused $(250 \mathrm{ml})$. Finally, the incidence of PVCs among our cardiac patients could be higher than in non-cardiac ICU patients. Our results are in accordance with those from other studies regarding the predictive value and limitations of dynamic indices. In addition, they demonstrate how these situations influence the predictive value of the dynamic indices in routine ICU practice.

In summary, although PPV, SPV, and SVV are reliable predictors of fluid responsiveness under strict conditions, they are not accurate predictors of volume responsiveness in mechanically ventilated patients after cardiac surgery in routine practice. This is caused by the preferent use of low TVs, presence of cardiac arrhythmias, and the applied calculation methods. Clinicians should be aware of these limitations when using dynamic indices for predicting volume responsiveness.

\section{References}

[1] D. Payen, A. C. de Pont, Y. Sakr, C. Spies, K. Reinhart, and J. L. Vincent, "A positive fluid balance is associated with a worse outcome in patients with acute renal failure," Crit Care, vol. 12, no. 3, p. R74, 2008.

[2] J. L. Vincent and M. H. Weil, "Fluid challenge revisited," Crit Care Med, vol. 34, no. 5, pp. 1333-7, 2006. 
[3] S. Brandt, T. Regueira, H. Bracht, F. Porta, S. Djafarzadeh, J. Takala, J. Gorrasi, E. Borotto, V. Krejci, L. B. Hiltebrand, L. E. Bruegger, G. Beldi, L. Wilkens, P. M. Lepper, U. Kessler, and S. M. Jakob, "Effect of fluid resuscitation on mortality and organ function in experimental sepsis models," Crit Care, vol. 13, no. 6, p. R186, 2009.

[4] A. L. Rosenberg, R. E. Dechert, P. K. Park, and R. H. Bartlett, "Review of a large clinical series: association of cumulative fluid balance on outcome in acute lung injury: a retrospective review of the ardsnet tidal volume study cohort," $J$ Intensive Care Med, vol. 24, no. 1, pp. 35-46, 2009.

[5] P. E. Marik, R. Cavallazzi, T. Vasu, and A. Hirani, "Dynamic changes in arterial waveform derived variables and fluid responsiveness in mechanically ventilated patients: a systematic review of the literature," Crit Care Med, vol. 37, no. 9, pp. 2642-7, 2009.

[6] F. Michard and J. L. Teboul, "Predicting fluid responsiveness in icu patients: a critical analysis of the evidence," Chest, vol. 121, no. 6, pp. 2000-8, 2002.

[7] F. Michard, S. Boussat, D. Chemla, N. Anguel, A. Mercat, Y. Lecarpentier, C. Richard, M. R. Pinsky, and J. L. Teboul, "Relation between respiratory changes in arterial pulse pressure and fluid responsiveness in septic patients with acute circulatory failure," Am J Respir Crit Care Med, vol. 162, no. 1, pp. $134-8,2000$.

[8] A. Kramer, D. Zygun, H. Hawes, P. Easton, and A. Ferland, "Pulse pressure variation predicts fluid responsiveness following coronary artery bypass surgery," Chest, vol. 126, no. 5, pp. 1563-8, 2004.

[9] S. T. Vistisen, J. J. Struijk, and A. Larsson, "Automated pre-ejection period variation indexed to tidal volume predicts fluid responsiveness after cardiac surgery," Acta Anaesthesiol Scand, vol. 53, no. 4, pp. 534-42, 2009.

[10] F. Michard, "Changes in arterial pressure during mechanical ventilation," Anesthesiology, vol. 103, no. 2, pp. 419-28, 2005.

[11] S. Magder, "Clinical usefulness of respiratory variations in arterial pressure," Am J Respir Crit Care Med, vol. 169, no. 2, pp. 151-5, 2004.

[12] S. Maguire, J. Rinehart, S. Vakharia, and M. Cannesson, "Technical communication: respiratory variation in pulse pressure and plethysmographic waveforms: intraoperative applicability in a north american academic center," Anesth Analg, vol. 112, no. 1, pp. 94-6, 2011. 
[13] B. Lansdorp, D. Ouweneel, A. de Keijzer, J. G. van der Hoeven, J. Lemson, and P. Pickkers, "Non-invasive measurement of pulse pressure variation and systolic pressure variation using a finger cuff corresponds with intra-arterial measurement," British journal of anaesthesia, vol. 107, no. 4, pp. 540-5, 2011.

[14] P. Q. Eichacker, E. P. Gerstenberger, S. M. Banks, X. Cui, and C. Natanson, "Meta-analysis of acute lung injury and acute respiratory distress syndrome trials testing low tidal volumes," Am J Respir Crit Care Med, vol. 166, no. 11, pp. 1510-4, 2002.

[15] T. D. Girard and G. R. Bernard, "Mechanical ventilation in ards: a state-of-theart review," Chest, vol. 131, no. 3, pp. 921-9, 2007.

[16] M. J. Schultz, J. J. Haitsma, A. S. Slutsky, and O. Gajic, "What tidal volumes should be used in patients without acute lung injury?" Anesthesiology, vol. 106, no. 6, pp. 1226-31, 2007.

[17] J. Akkermans, M. Diepeveen, W. Ganzevoort, G. A. van Montfrans, B. E. Westerhof, and H. Wolf, "Continuous non-invasive blood pressure monitoring, a validation study of nexfin in a pregnant population," Hypertens Pregnancy, vol. 28, no. 2, pp. 230-42, 2009.

[18] D. W. Eeftinck Schattenkerk, J. J. van Lieshout, A. H. van den Meiracker, K. R. Wesseling, S. Blanc, W. Wieling, G. A. van Montfrans, J. J. Settels, K. H. Wesseling, and B. E. Westerhof, "Nexfin noninvasive continuous blood pressure validated against riva-rocci/korotkoff," Am J Hypertens, vol. 22, no. 4, pp. 37883, 2009.

[19] L. N. Diebel, R. F. Wilson, M. G. Tagett, and R. A. Kline, "End-diastolic volume. a better indicator of preload in the critically ill," Archives of surgery, vol. 127, no. 7, pp. 817-21; discussion 821-2, 1992.

[20] M. R. Lopes, M. A. Oliveira, V. O. Pereira, I. P. Lemos, J. Auler, J. O., and F. Michard, "Goal-directed fluid management based on pulse pressure variation monitoring during high-risk surgery: a pilot randomized controlled trial," Crit Care, vol. 11, no. 5, p. R100, 2007.

[21] F. Michard, D. Chemla, C. Richard, M. Wysocki, M. R. Pinsky, Y. Lecarpentier, and J. L. Teboul, "Clinical use of respiratory changes in arterial pulse pressure to monitor the hemodynamic effects of peep," Am J Respir Crit Care Med, vol. 159, no. 3, pp. 935-9, 1999.

[22] S. T. Vistisen, J. Koefoed-Nielsen, and A. Larsson, "Should dynamic parameters for prediction of fluid responsiveness be indexed to the tidal volume?" Acta Anaesthesiol Scand, 2009. 
[23] Y. Sakr, J. L. Vincent, K. Reinhart, J. Groeneveld, A. Michalopoulos, C. L. Sprung, A. Artigas, and V. M. Ranieri, "High tidal volume and positive fluid balance are associated with worse outcome in acute lung injury," Chest, vol. 128, no. 5, pp. 3098-108, 2005.

[24] H. K. Kim and M. R. Pinsky, "Effect of tidal volume, sampling duration, and cardiac contractility on pulse pressure and stroke volume variation during positive-pressure ventilation," Crit Care Med, vol. 36, no. 10, pp. 2858-62, 2008 .

[25] D. A. Reuter, J. Bayerlein, M. S. Goepfert, F. C. Weis, E. Kilger, P. Lamm, and A. E. Goetz, "Influence of tidal volume on left ventricular stroke volume variation measured by pulse contour analysis in mechanically ventilated patients," Intensive Care Med, vol. 29, no. 3, pp. 476-80, 2003.

[26] C. Charron, C. Fessenmeyer, C. Cosson, J. X. Mazoit, J. L. Hebert, D. Benhamou, and A. R. Edouard, "The influence of tidal volume on the dynamic variables of fluid responsiveness in critically ill patients," Anesth Analg, vol. 102, no. 5, pp. 1511-7, 2006.

[27] D. De Backer, S. Heenen, M. Piagnerelli, M. Koch, and J. L. Vincent, "Pulse pressure variations to predict fluid responsiveness: influence of tidal volume," Intensive Care Med, vol. 31, no. 4, pp. 517-23, 2005.

[28] ARDS-network, "Ventilation with lower tidal volumes as compared with traditional tidal volumes for acute lung injury and the acute respiratory distress syndrome. the acute respiratory distress syndrome network," $N$ Engl J Med, vol. 342, no. 18, pp. 1301-8, 2000.

[29] S. T. Vistisen, J. Koefoed-Nielsen, and A. Larsson, "Automated pre-ejection period variation predicts fluid responsiveness in low tidal volume ventilated pigs," Acta Anaesthesiol Scand, 2009.

[30] X. Monnet, M. Rienzo, D. Osman, N. Anguel, C. Richard, M. R. Pinsky, and J. L. Teboul, "Passive leg raising predicts fluid responsiveness in the critically ill," Crit Care Med, vol. 34, no. 5, pp. 1402-7, 2006.

[31] S. W. Thiel, M. H. Kollef, and W. Isakow, "Non-invasive stroke volume measurement and passive leg raising predict volume responsiveness in medical icu patients: an observational cohort study," Crit Care, vol. 13, no. 4, p. R111, 2009. 
[32] B. Lamia, A. Ochagavia, X. Monnet, D. Chemla, C. Richard, and J. L. Teboul, "Echocardiographic prediction of volume responsiveness in critically ill patients with spontaneously breathing activity," Intensive Care Med, vol. 33, no. 7, pp. 1125-32, 2007.

[33] M. I. Monge Garcia, A. Gil Cano, and J. C. Diaz Monrove, "Arterial pressure changes during the valsalva maneuver to predict fluid responsiveness in spontaneously breathing patients," Intensive Care Med, vol. 35, no. 1, pp. 77-84, 2009.

[34] S. Rehberg, C. Ertmer, and M. Westphal, "Valsalva, valsalva, may you give me a clue, who needs fluids in my icu?" Intensive Care Med, vol. 35, no. 1, pp. $7-8,2009$.

[35] X. Monnet, D. Osman, C. Ridel, B. Lamia, C. Richard, and J. L. Teboul, "Predicting volume responsiveness by using the end-expiratory occlusion in mechanically ventilated intensive care unit patients," Crit Care Med, vol. 37, no. 3, pp. 951-6, 2009.

[36] S. Preisman, S. Kogan, H. Berkenstadt, and A. Perel, "Predicting fluid responsiveness in patients undergoing cardiac surgery: functional haemodynamic parameters including the respiratory systolic variation test and static preload indicators," Br J Anaesth, vol. 95, no. 6, pp. 746-55, 2005.

[37] H. Solus-Biguenet, M. Fleyfel, B. Tavernier, E. Kipnis, J. Onimus, E. Robin, G. Lebuffe, C. Decoene, F. R. Pruvot, and B. Vallet, "Non-invasive prediction of fluid responsiveness during major hepatic surgery," Br J Anaesth, vol. 97, no. 6, pp. 808-16, 2006. 


\title{
4 \\ Ventilator induced pulse pressure variation in neonates
}

\author{
L. Heskamp, B. Lansdorp, J. Hopman, J. Lemson, W.P de Boode. \\ Submitted: Pediatric Research
}

\begin{abstract}
Background. During positive pressure ventilation, arterial pressure variations, like the pulse pressure variation (PPV), are observed in neonates. However, the frequency of the PPV does not always correspond with the respiratory rate. It is hypothesized that PPV is caused by cardiopulmonary interaction, but that this mismatch is related to the low respiratory rate/heart rate ratio. Therefore, the goal of this study is to investigate the relation between PPV and ventilation in neonates.

Methods. The arterial blood pressure was continuously monitored in twenty neonates on synchronized intermittent mandatory ventilation (SIMV) and ten neonates on high frequency ventilation (HFV). In SIMV ventilated neonates the $\mathrm{CO} 2$ waveform and in $\mathrm{HFV}$ ventilated neonates the thorax impedance waveform was continuously monitored and defined as the respiratory signal. Correlation and coherence between the respiratory signal and PP were determined.

Results. Correlation between the respiratory signal and PP was $-0.64 \pm 0.18$ and $0.55 \pm 0.16$ and coherence at the respiratory frequency was $0.95 \pm 0.11$ and $0.76 \pm 0.4$ for SIMV and HFV, respectively. Conclusions. The arterial pressure variations observed in neonates on SIMV or HFV are related to cardiopulmonary interaction. Despite this relation, it is not likely that PPV will reliable predict fluid responsiveness in neonates.
\end{abstract}




\subsection{Introduction}

To ensure adequate end-organ perfusion and tissue oxygenation in neonates an adequate filling pressure (preload) is necessary [1]. Therefore, in case of true hypovolemia, a fluid bolus can be life-saving. However, volume expansion in an already hypervolemic neonate is associated with an adverse neurological outcome [2], increased prevalence of chronic lung disease [3], and increased mortality [4]. To avoid these adverse effects it is important to determine if a neonate will increase stroke volume when a fluid bolus is given, i.e. will be fluid responsive. Studies show that currently used clinical and static hemodynamic parameters are not able to accurately predict fluid responsiveness in neonates $[1,5]$. In adults, it is shown that dynamic indices based on preload changes secondary to cardiopulmonary interaction during positive pressure ventilation can predict fluid responsiveness [6-8]. According to the Frank-Starling relation, these ventilator-induced preload changes result in variation in stroke volume [9-11] and the proposed surrogate of stroke volume, the arterial pulse pressure (PP) [11]. Marik et al. [12] reported in a meta-analysis that pulse pressure variation (PPV) predicts fluid responsiveness with a sensitivity of $89 \%$ and specificity of $88 \%$ in the adult population under specific conditions.

In clinical practice PPV is also observed in neonates. However, the frequency of these variations does not always correspond with the respiratory rate (RR). The observed mismatch in frequencies in neonates might be explained by the low heart rate to respiratory rate $(\mathrm{HR} / \mathrm{RR}$ ) ratio (due to a relative higher basal $\mathrm{RR}$ than heart rate (HR)). Also in adult patients, it has been shown that the absolute and predictive value of PPV is diminished when the HR/RR ratio is low [13]. Because of this observed mismatch, it is important to firstly determine whether the observed PPV in neonates is indeed related to cardiopulmonary interaction before investigating the ability of PPV to predict fluid responsiveness in neonates. To our knowledge, there are no studies that demonstrated this relation. Therefore, the goal of this study is to investigate the relation between PPV and cardiopulmonary interaction in neonates ventilated with the two most common modes of ventilation, i.e. synchronized intermittent mandatory ventilation (SIMV) and high frequency ventilation (HFV).

\subsection{Study population}

This study was performed in (preterm) neonates admitted to the neonatology intensive care unit of the Radboud university medical centre. Inclusion criteria were the presence of an intra-arterial catheter and mechanical ventilation with SIMV or HFV (Leoni Plus, Heinen \& Lowenstein, Bad Ems, Germany), and absence of spontaneous breathing activity. 


\subsubsection{Data acquisition}

Invasive arterial blood pressure (ABP) was continuously measured using an arterial catheter. In patient on SIMV, the $\mathrm{CO}_{2}$ waveform was continuously measured using the capnograph and in patients on HFV the thoracic impedance waveform was continuously measured. The $\mathrm{CO} 2$ waveform or thoracic impedance waveform were defined as the respiratory signal (Resp). ABP and Resp were acquired via the IntelliVue Patient Monitor (MP90, Philips Healthcare, Best, The Netherlands) and recorded with Trendface (ixellence, Wildau, Germany) with a sampling frequency of $62.5 \mathrm{~Hz}$.

\subsubsection{Data analysis}

Two minutes of recording without artefacts in ABP and Resp were manually selected for further processing and analysis. The systolic and diastolic pressures were detected using a peak detection algorithm in Matlab (Matlab R2011A, MathWorks Inc., Natick, MA, USA). Pulse pressure was defined as the difference between the systolic and the preceding diastolic pressure, as depicted in Figure 1a. In order to simulate the effect that the respiratory cycle is sampled with the heart rate (because the effect of the ventilation on the circulation is expressed per heartbeat) and to determine whether the PPV is caused by cardiopulmonary interaction, the value of the respiratory signal was taken at the moment the systolic pressure of a heartbeat was reached, as illustrated in Figure 1a and 1b by $\operatorname{Resp}_{H R}$.

\section{Frequency spectra}

Frequency spectra were obtained from Resp, $\operatorname{Resp}_{H R}$ and PP with a Fourier Transform. However, the Fourier Transform can only be applied if the time-series have evenly spaced samples. Because PP and RespHR have an irregular sampling frequency, namely heart rate (HR), in both time-series the samples are unevenly spaced. To obtain evenly spaced time-series, PP and Resp $\mathrm{p}_{H R}$ were linear interpolated and resampled at $10 \mathrm{~Hz}$, see Figure 1c and 1d. Frequency spectra were obtained using Welchs method with a 30 second window and $50 \%$ overlap (Figure 1e, 1f and 1g).

\section{Effect of the low HR/RR ratio}

principle of aliasing

It is hypothesized that the observed mismatch in frequencies in neonates can be explained by the HR/RR ratio seen in neonates. This is because at least two samples (in this case heartbeats) per respiratory cycle are needed to adequately represent the respiratory signal and to avoid undersampling (Nyquist-Shannon sampling theorem [14]). As depicted in Figure 2a, if there are for example three heartbeats per respiratory cycle, the respiratory signal is adequately represented as a sinus of $1 \mathrm{~Hz}$. 
However, when there are less than two heartbeats per respiratory cycle, undersampling occurs, wherefore the respiratory signal is not represented as the original sinus of $1 \mathrm{~Hz}$, but as a sinus with a lower frequency (Figure $2 \mathrm{~b}$ ). This phenomenon is called aliasing and this lower frequency is called the alias frequency. Hence, if the respiratory signal is undersampled due to a HR/RR ratio below $2, \operatorname{Resp}_{H R}$ will not vary with $R R$, but the so called alias frequency of $R R\left(R_{\text {alias }}\right)$.

\section{aliasing in $\mathrm{HFV}$}

During HFV, the HR/RR ratio is always smaller than 2. This indicates that aliasing of $\mathrm{RR}$ will occur. $\mathrm{RR}_{\text {alias }}$ can be predicted using equation 1 with $\mathrm{n}$ the closest integer multiple of HR to RR.

$$
\mathrm{RR}_{\text {alias }}=|\mathrm{nHR}-\mathrm{RR}|
$$

RRalias was compared to the observed frequency in $\operatorname{Resp}_{H R}$ and PP. If PP varies with $\mathrm{RR}_{\text {alias }}$, this will support the hypothesis that in neonates on HFV PPV is caused by cardiopulmonary interaction and that the mismatch between the frequency in PP and $\mathrm{RR}$ is due to the HR/RR ratio smaller than 2 .

\section{Aliasing in SIMV}

Generally, in patients ventilated with SIMV without spontaneous breathing activity the HR/RR ratio is not smaller than 2. Hence, aliasing of RR will not occur. However, Resp is not a pure sinus. Therefore, Resp does not only contain the fundamental frequency RR, but also higher frequencies from which the multiples of RR (2RR, $3 R R$, etc.) are the most prominent, see Figure 1e. The ratio between HR and these higher frequencies (e.g. 2RR and 3RR) can be smaller than 2. Therefore, aliasing still occurs even with a HR/RR ratio larger than 2 . This means, when sampling Resp with HR, it is expected that in the frequency spectrum of $\operatorname{Resp}_{H R}$ alias frequencies of these higher frequencies (e.g. alias frequencies of $2 R R$ and 3RR) will be observed, see figure 1f. If these alias frequencies are also observed in PP, as illustrated in Figure 1g, this will support the hypothesis that in neonates on SIMV PPV is caused by cardiopulmonary interaction and that the presence of frequencies in PP not equal to $\mathrm{RR}$ is caused by the low $\mathrm{HR} / \mathrm{RR}$ ratio.

\section{Relation between ventilation and PPV}

The relation between ventilation and PP was determined in both the time-domain and frequency domain. In the time-domain, the Spearman correlation was determined between PP and Resp $\mathrm{P}_{H R}$, while correcting for the time-delay between PP and Resp $\mathrm{P}_{H R}$. For SIMV, the maximum negative correlation was selected because during inspiration Resp decreases $\left(\mathrm{CO}_{2}\right.$ decreases) while PP increases. For HFV, the maximum 

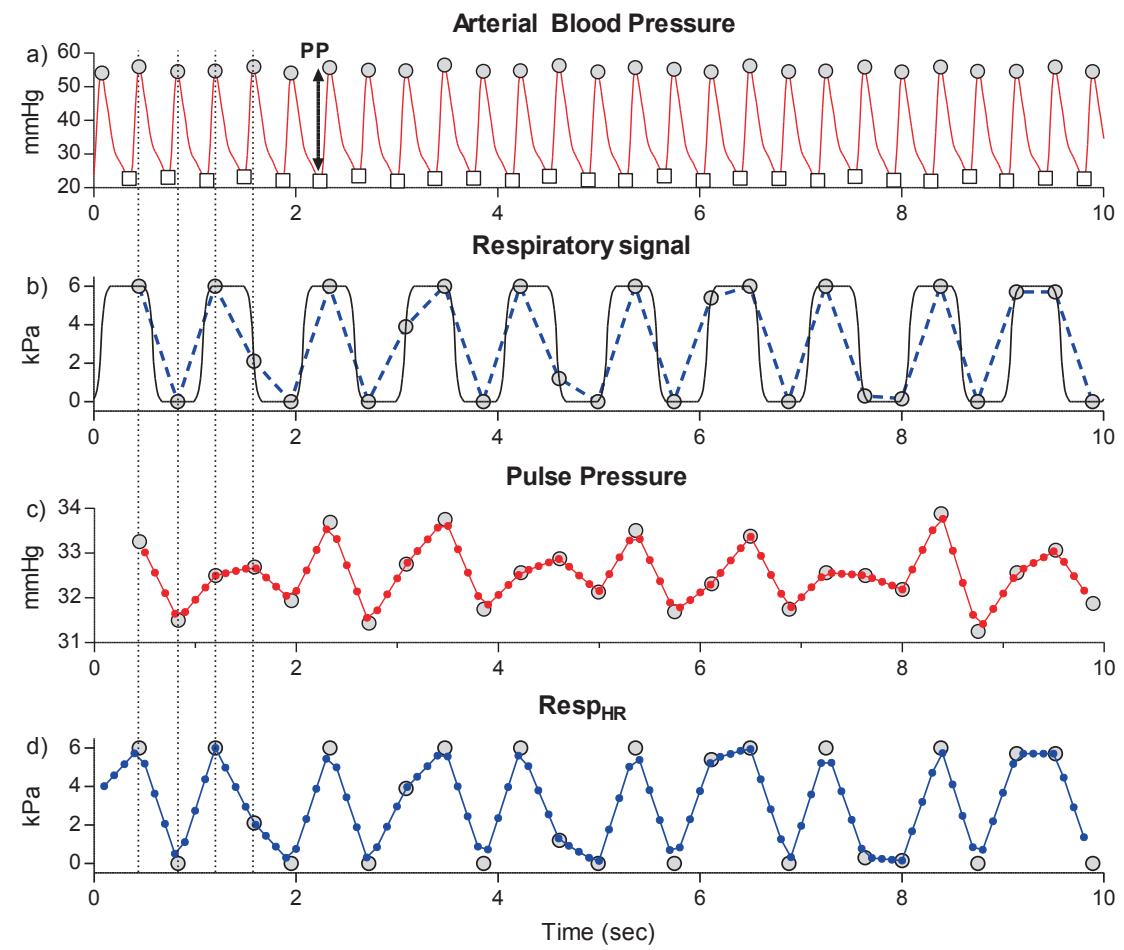

Frequency Spectra

Respiratory signal

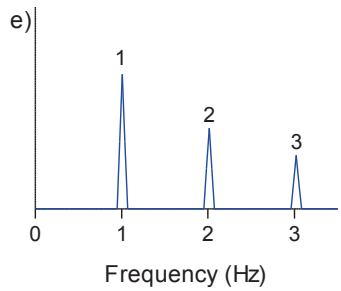

$\operatorname{Resp}_{\mathrm{HR}}$

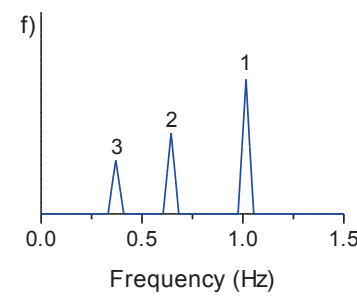

Pulse Pressure

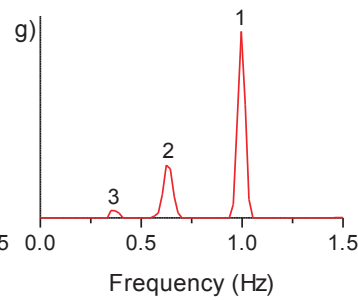

Figure 1: Representation of the steps performed during the data analysis $(\mathrm{HR}=2.7 \mathrm{~Hz}$, SIMV with RR = $1 \mathrm{~Hz}$ ). a) Arterial blood pressure with systolic (grey circle) and diastolic blood pressure (white square). b) Respiratory signal $\left(\mathrm{CO}_{2}\right.$, solid black line), respiratory signal values obtained at the moment that the systolic pressure is reached (grey circles) and RespHR (blue dashed line). $c$ and d) PP and Resp ${ }_{H R}$. The grey circles depict PP and the respiratory value at the moment systolic pressure was reached, respectively. The red and blue lines depict the interpolated PP and Resp $p_{H R}$ with the red and blue dots the interpolated samples, respectively. e) Frequency spectrum of the respiratory signal containing: $R R(1)$, $2 R R(2)$ and $3 R R(3) . f$ and $g$ ) Frequency spectrum of RespHR and PP containing $R R(1)$ and the alias frequencies of $2 R R$ and $3 R R$ ( 2 and 3 , respectively). $H R=$ heart rate, $R R=$ respiratory rate, $P P=$ pulse pressure, $\operatorname{Resp}_{H R}=$ the respiratory values at the moment systolic pressure was reached. 


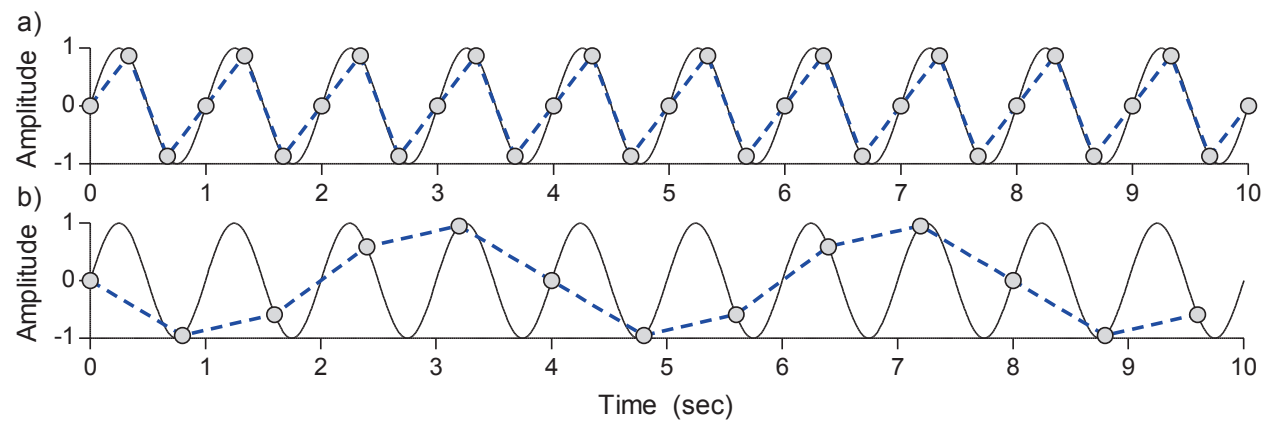

Figure 2: Aliasing: The black solid line is a sinus of $1 \mathrm{~Hz}$ and represents the respiratory signal; the grey circles are the obtained samples (representing the heartbeats). The blue dashed line is the reconstructed sinus (based on the grey circles), representing Resp $p_{H R}$. a) $R R=1 \mathrm{~Hz}$ and $\mathrm{HR}=3 \mathrm{~Hz}$, the respiratory signal is adequately represented as sinus of $1 \mathrm{~Hz}$. b) $R R=1 \mathrm{~Hz}$ and $H R=1.25 \mathrm{~Hz}$, the sinus is undersampled which results in a reconstructed sinus with a lower frequency, in this case $0.25 \mathrm{~Hz}$ (the alias frequency). $R R=$ respiratory rate, $H R=$ heart rate.

positive correlation was selected because during inspiration Resp increases (thoracic impedance increases) while PP increases. In the frequency-domain, the coherence function was determined between PP and Resp $\mathrm{R}_{H R}$. Briefly, the coherence function is the correlation determined for each frequency region. The coherence function ranges between 0 and 1 (no relation and perfect relation, respectively). The coherence value was determined at RR for SIMV and the observed alias frequency in $\operatorname{Resp}_{H R}$ for HFV. A relation between ventilation and PP was presumed when coherence reached the cut-off value for significant coherence. The cut-off value was 0.42 for SIMV and 0.46 for HFV [15].

\subsection{Results}

\subsubsection{Patient characteristics}

For SIMV, 20 patients were included with a median gestational age of 33 weeks (IQR 29-39 weeks), a median postnatal age of 8 days (IQR 2-13 days) and a median birth weight of 1745 gram (IQR 1119-3238 gram). For HFV, 10 patients were included with a median gestational age of 26 weeks (IQR 24-31 weeks), a median postnatal age of 16 days (IQR 10-21 days) and a median birth weight of 1010 gram (IQR 8051980 gram). The position of the arterial catheter varied between the left and right radial and tibial artery and the umbilical artery. The HR/RR ratio was $2.6 \pm 0.5$ and $0.4 \pm 0.1$ for neonates on SIMV and HFV, respectively. 


\subsubsection{HFV}

\section{Time-domain and frequency domain}

Figure 3 depicts Resp, RespHR and PP in the time-domain and frequency-domain for a patient supported with HFV to illustrate the results $(\mathrm{RR}=6 \mathrm{~Hz}, \mathrm{HR}=2.7 \mathrm{~Hz})$. First, in the time-domain PPV is observed (Figure 3c). In the frequency-domain it is observed that the frequency of this PPV equals the frequency observed in Resp $\mathrm{P}_{H R}$ (Figure 3e). Second, also a variation in PP with frequency $<0.1 \mathrm{~Hz}$ is observed that is not observed in $\operatorname{Resp}_{H R}$, representing the Mayer waves (Figure 3e). The findings illustrated in Figure 3 are observed in all patients ventilated with HFV.

\section{Aliasing}

Table 1 shows RRalias, the frequency in RespHR and the frequency in PP for all patients ventilated with HFV. There is no difference observed between $\mathrm{RR}_{\text {alias }}$, the frequency in $\operatorname{Resp}_{H R}$ and the frequency in PP.

Table 1: Alias frequency of $R R\left(R R_{\text {alias }}\right)$ and the observed frequency in Resp $p_{H R}$ and pulse pressure for patients ventilated with HFV. Resp $p_{H R}=$ respiratory signal at the moment of systolic pressure is reached.

\begin{tabular}{||l|l|l|l|l||}
\hline Patient & HR/RR ratio & RR $_{\text {alias }}$ & Frequency in Resp $\mathrm{P}_{H R}$ & Frequency in PP \\
\hline 1 & 0.45 & 0.57 & 0.55 & 0.55 \\
2 & 0.55 & 0.60 & 0.63 & 0.63 \\
3 & 0.41 & 1.20 & 1.19 & 1.17 \\
4 & 0.36 & 0.60 & 0.64 & 0.64 \\
5 & 0.23 & 0.73 & 0.72 & 0.72 \\
6 & 0.24 & 0.60 & 0.61 & 0.45 \\
7 & 0.34 & 0.15 & 0.23 & 0.20 \\
8 & 0.31 & 0.75 & 0.66 & 0.66 \\
9 & 0.27 & 0.73 & 0.84 & 0.84 \\
10 & 0.29 & 1.30 & 1.19 & 1.27 \\
\hline
\end{tabular}

\section{Relation between ventilation and PPV}

The Spearman correlation between $\operatorname{Resp}_{H R}$ and PP, while correcting for the timedelay, was $0.55 \pm 0.16$. The coherence value between Resp and PP at $\mathrm{RR}_{\text {alias }}$ was $0.76 \pm 0.4$. Only in two patients the coherence value was not significant. 

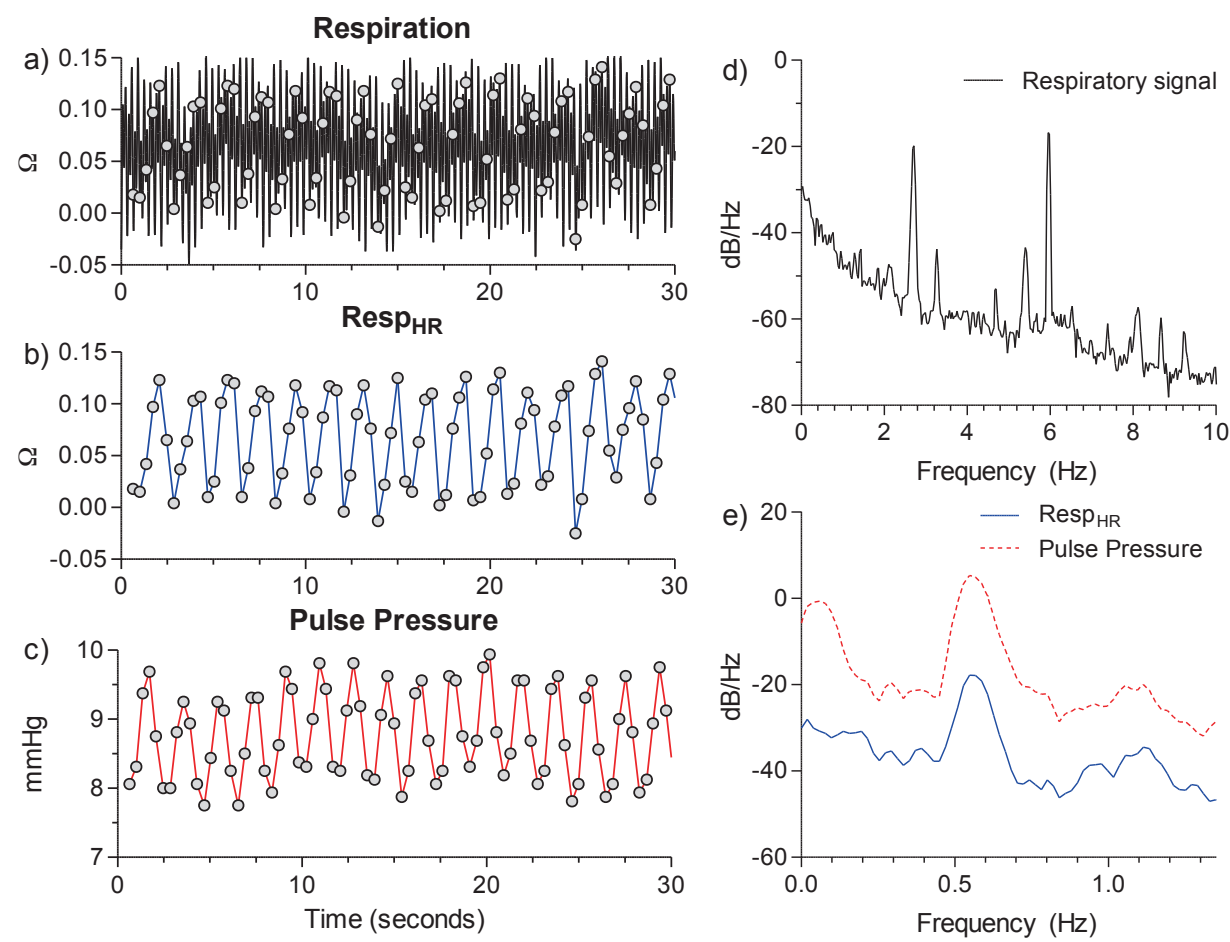

Figure 3: Respiratory signal, Resp $p_{H R}$ and pulse pressure in the time and frequency domain for an example patient ventilated with $\mathrm{HFV}(\mathrm{RR}=6 \mathrm{~Hz}, \mathrm{HR}=2.7 \mathrm{~Hz}$. a) Respiratory signal with time-points that systolic pressure is reached (grey circles). b) Resp ${ }_{H R}$. c) Pulse pressure. d) Frequency spectrum of the respiratory signal. e) Frequency spectra of RespHR (solid blue) and pulse pressure (dashed red). $R R=$ respiratory rate, $H R=$ heart rate. $R_{e s p} p_{H R}=$ the respiratory values at the moment systolic pressure was reached. 

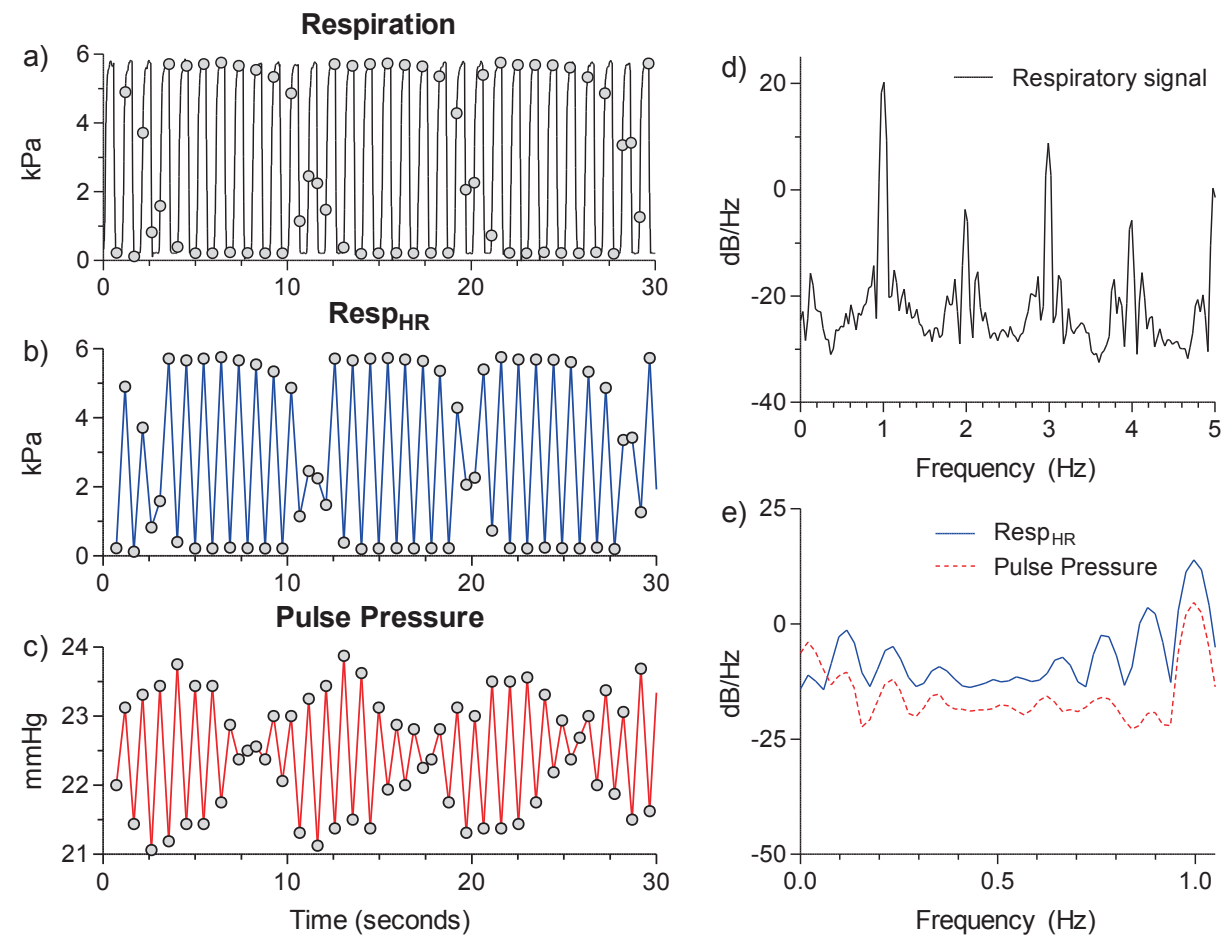

Figure 4: Respiratory signal, RespHR and pulse pressure in the time and frequency domain for an example patient ventilated with $\operatorname{SIMV}(R R=1 \mathrm{~Hz}, \mathrm{HR}=2.1 \mathrm{~Hz}$. a) Respiratory signal with time-points that systolic pressure is reached (grey circles). b) Resp $p_{H R}$. c) Pulse pressure. d) Frequency spectrum of the respiratory signal. e) Frequency spectra of RespHR (solid blue) and pulse pressure (dashed red). $R R=$ respiratory rate, $H R=$ heart rate. $R_{e s p} p_{H R}=$ the respiratory values at the moment systolic pressure was reached. 


\subsubsection{SIMV}

\section{Time-domain and frequency domain}

Figure 4 depicts Resp, $\operatorname{Resp}_{H R}$ and PP in the time-domain and frequency-domain for a patient ventilated with SIMV to illustrate the results $(\mathrm{RR}=1 \mathrm{~Hz}, \mathrm{HR}=2.1 \mathrm{~Hz})$. First, in the time-domain it is seen that, PP varies with inspiration and expiration, as expected (Figure 4c). In the frequency domain it is observed that this variation coincides with RR (Figure 4d and 4e). Second, waves with lower frequencies are observed in PP that are also observed in $\operatorname{Resp}_{H R}$ (Figure $4 \mathrm{~b}, 4 \mathrm{c}$ and $4 \mathrm{e}$ ). These additional lower frequencies equal the alias frequencies of the harmonic frequencies of RR. Third, a variation in PP with a frequency $i 0.1 \mathrm{~Hz}$ is observed that is not observed in $\operatorname{Resp}_{H R}$ (Mayer waves) (Figure 4e). The findings illustrated in Figure 4 are observed in all patients on SIMV.

\section{Relation between ventilation and PPV}

The Spearman correlation between RespHR and PP was $-0.64 \pm 0.18$. The coherence value between $\operatorname{Resp}_{H R}$ and PP at RR was $0.95 \pm 0.11$. Coherence was significant in all patients.

\subsection{Discussion}

Our study shows that in neonates PPV can be observed during both SIMV and HFV and that it is related to cardiopulmonary interaction. In neonates on HFV it is observed that the frequency with which PP varies, coincidences with the alias frequency of RR. In neonates ventilated with SIMV the frequencies in PP were equal to RR or the alias frequencies of the multiples of RR. These findings support the hypothesis that the mismatch observed between RR and the frequencies in PP are caused by the low HR/RR ratio $(2.6 \pm 0.5$ and $0.4 \pm 0.1$ for neonates on SIMV and HFV, respectively).

\subsubsection{Effect of low $H R / R R$ ratio}

The finding that the PP varies with the alias frequencies observed in RespHR suggests that PPV in neonates is caused by physiological aliasing. Rother et al. [16] and Witte et al. [17] already described this phenomenon during heart rate variability analysis. However when a closer look is taken at the origin of PPV it is believed that not physiological aliasing, but another mechanism underlies this phenomenon. Cardiopulmonary interaction is a dynamic system that consists of the ABP with a frequency HR and the ventilation with a frequency RR, where ABP is influenced by the ventilation. It is known that when RR becomes close to HR, ABP will not only 
vary with HR but also with a frequency equal to the difference between HR and RR, i.e. the difference frequency $[18,19]$. The occurrence of this difference frequency can be visualized by the interaction of two sinuses, as depicted in Figure 5. Mitzner et al. [18] showed in a dog model that the difference frequency was observed in the pulmonary artery pressure, aorta pressure and also in the right ventricle stroke volume. In our situation the difference frequency equals the alias frequency, because the sampling frequency is equal to HR:

difference frequency $=\mid$ HR-RR $\mid$, alias frequency $=\mid$ fs-RR $\mid$ with fs $=H R$.

Interestingly, Mitzner et al. [18] showed in their dog model that not only the difference frequency between RR and HR was observed, but also between RR and multiples of HR. Therefore, it is hypothesized that in neonates on HFV the interaction between RR and the closest multiple of HR causes the observed PPV. If this hypothesis is true, the difference frequency should already be present in the original ABP signal. If retrospectively the frequency spectrum of $\mathrm{ABP}$ is obtained, it is indeed observed that the difference frequency between RR and the closest multiple of HR was present in $\mathrm{ABP}$. Because the original $\mathrm{ABP}$ signal was sampled with a frequency of $62.5 \mathrm{~Hz}$ and $\mathrm{RR}$ is always lower than half this sampling frequency (smaller than $31.25 \mathrm{~Hz}$ ), there is no undersampling in the original ABP signal and therefore no aliasing. Hence, it is more likely that the difference frequency instead of physiological aliasing explains the presence of PPV in neonates on HFV. In neonates ventilated with SIMV it is also believed that difference frequency instead of physiological aliasing explains the frequencies in PP that did not correspond to RR. If in these patients a frequency spectrum of the original ABP signal is obtained, it is indeed observed that the frequencies in PP not equal to RR are already observed in the original ABP signal. Hence, also in neonates on SIMV it is most likely that the difference frequency explains the variation in PP at frequencies unequal to RR. During both HFV and SIMV the difference frequencies plays a role in the occurrence of the frequencies with which PP varies. However, because during HFV there is not even one heartbeat per respiratory cycle, it is possible that the underlying mechanism causing PPV is not exactly the same. The occurrence of these difference frequencies in the PP of the neonates cannot be ignored, because they influence the calculated PPV. In adults PPV is defined as the relative difference in maximal and minimal PP within one respiratory cycle. However, as clearly visible in Figure 4, the calculated PPV will depend on which respiratory cycle is used to determine PPV.

\subsubsection{PPV as predictor for fluid responsiveness}

PPV can be used to predict fluid responsiveness in adult patients under certain con-

ditions $[8,12]$. The finding that PPV is also observed in neonates and related to 


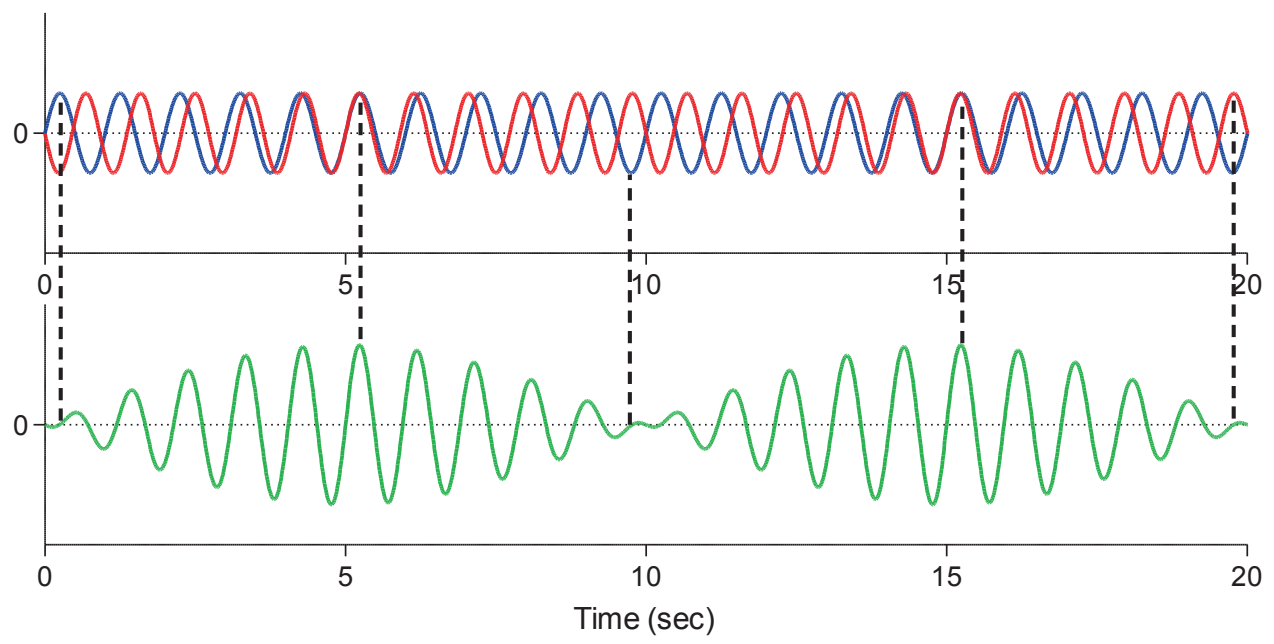

Figure 5: Interference between two sinuses (1 Hz (blue), $1.1 \mathrm{~Hz}$ (red)). The difference frequency $(0.1 \mathrm{~Hz})$ is observed in the resulting sinus (green).

cardiopulmonary interaction supports the hypothesis that PPV might be able to predict fluid responsiveness in neonates. However, the difference in physiology between adults and neonates is of major influence on the interpretation of PPV as an indicator of fluid responsiveness. As shown in this study, the low HR/RR ratio observed in neonates results in the presence of difference frequencies, which impedes the analysis and interpretation of PPV in neonates. In adults it is already known that the predictive value of PPV is limited with a HR/RR ratio smaller than 3.6 [13]. However, they do not report the presence of the difference frequencies as observed in our study with low HR/RR ratio. In addition, other physiological characteristics might also negatively influence the predictive value of PPV in neonates. First, it is believed that in mechanically ventilated neonates the intrathoracic pressure variations are small due to a higher thoracic compliance [20], ventilation with low tidal volumes (4-6 ml/kg) or ventilation with high frequency ventilation (HFV). In adults, it is known that the predictive value of PPV is limited with smaller intrathoracic pressure variation [2123]. Second, neonates have a larger arterial compliance in comparison with adults [24] that may attenuate arterial blood pressure variation and hence PPV.

Another limitation of PPV is that it can only be applied under specific conditions, as is known from adults [25]. PPV can only predict fluid responsiveness when patients are ventilated with positive pressure ventilation and have no spontaneous breathing activity [26]. During spontaneous breathing activity the intrathoracic pressure variation are of irregular rate and amplitude, which influences PPV. In adults, this spontaneous breathing activity is associated with loss of predictive value for PPV 
[26]. Lansdorp et al. [27] showed that PPV is not able to predict fluid responsiveness in the adult ICU during routine clinical practice. In accordance with the adult ICU, at the NICU main policy is also to avoid mechanical ventilation unless it is really necessary, to keep sedation as low as possible and to promote spontaneous breathing. As a consequence, also at the NICU only a small population is mechanically ventilated without spontaneous breathing activity. It is desirable to have a technique that can be applied in a large population. Recently the passive leg raise test [28, 29] and measurement of mean systemic filling pressure $[30,31]$ are proposed as promising techniques for predicting fluid responsiveness in critically ill adult patients. It will be interesting to investigate the applicability of these techniques in neonates. The passive leg raising test has already been investigated in children and could predict fluid responsiveness with a sensitivity of 55\% and specificity of $85 \%$ [32]. However, this was not in a neonatal population (median age 6 years).

\subsubsection{Limitations}

In this study only a small population was studied. Nevertheless, because the population varied widely in gestational age, postnatal age and underlying pathology and the results were consistent for this widely varying population, the validity of our results for other neonates on the NICU ventilated with SIMV or HFV is supported. Furthermore, no beat-to-beat measurement of SV was performed. Therefore, it cannot be validated whether the variation in PP at the difference frequencies is the results of variation in SV at the difference frequencies or if there is another underlying mechanism. A third limitation of this study is that we did not determine the predictive value of PPV for fluid responsiveness in neonates. Therefore, the effect of the observed difference frequencies on the predictive value of PPV for fluid responsiveness in the newborn infant ventilated with SIMV or HFV is unknown.

\subsection{Conclusion}

The observed PPV in neonates ventilated with the two most common modes of ventilation on NICU, SIMV and HFV, is related to positive pressure ventilation. This supports the possibility to use PPV as a predictor of fluid responsiveness in neonates. However, the occurrence of the difference frequency in PP impedes the analysis of PPV. Therefore, it is doubtful whether PPV will be a reliable predictor of fluid responsiveness in neonates. Hence, it is recommended to focus further research on other indicators of fluid responsiveness. 


\section{References}

[1] N. Evans, "Volume expansion during neonatal intensive care: do we know what we are doing?" Seminars in neonatology: SN, vol. 8, no. 4, pp. 315-23, 2003.

[2] A. Greenough, P. Cheeseman, V. Kavvadia, G. Dimitriou, and M. Morton, "Colloid infusion in the perinatal period and abnormal neurodevelopmental outcome in very low birth weight infants," European journal of pediatrics, vol. 161, no. 6, pp. 319-23, 2002.

[3] L. J. Van Marter, M. Pagano, E. N. Allred, A. Leviton, and K. C. Kuban, "Rate of bronchopulmonary dysplasia as a function of neonatal intensive care practices," The Journal of pediatrics, vol. 120, no. 6, pp. 938-46, 1992.

[4] A. K. Ewer, W. Tyler, A. Francis, D. Drinkall, and J. O. Gardosi, "Excessive volume expansion and neonatal death in preterm infants born at 27-28 weeks gestation," Paediatric and perinatal epidemiology, vol. 17, no. 2, pp. 180-6, 2003.

[5] W. P. de Boode, "Clinical monitoring of systemic hemodynamics in critically ill newborns," Early human development, vol. 86, no. 3, pp. 137-41, 2010.

[6] M. Feissel, F. Michard, I. Mangin, O. Ruyer, J. P. Faller, and J. L. Teboul, "Respiratory changes in aortic blood velocity as an indicator of fluid responsiveness in ventilated patients with septic shock," Chest, vol. 119, no. 3, pp. 867-73, 2001.

[7] F. Michard, S. Boussat, D. Chemla, N. Anguel, A. Mercat, Y. Lecarpentier, C. Richard, M. R. Pinsky, and J. L. Teboul, "Relation between respiratory changes in arterial pulse pressure and fluid responsiveness in septic patients with acute circulatory failure," Am J Respir Crit Care Med, vol. 162, no. 1, pp. 134-8, 2000.

[8] F. Michard and J. L. Teboul, "Predicting fluid responsiveness in icu patients: a critical analysis of the evidence," Chest, vol. 121, no. 6, pp. 2000-8, 2002.

[9] F. Michard, "Changes in arterial pressure during mechanical ventilation," Anesthesiology, vol. 103, no. 2, pp. 419-28, 2005.

[10] M. Cannesson, M. Aboy, C. K. Hofer, and M. Rehman, "Pulse pressure variation: where are we today?" Journal of clinical monitoring and computing, vol. 25, no. 1, pp. 45-56, 2011. 
[11] F. Jardin, J. C. Farcot, P. Gueret, J. F. Prost, Y. Ozier, and J. P. Bourdarias, "Cyclic changes in arterial pulse during respiratory support," Circulation, vol. 68 , no. 2, pp. 266-74, 1983.

[12] P. E. Marik, R. Cavallazzi, T. Vasu, and A. Hirani, "Dynamic changes in arterial waveform derived variables and fluid responsiveness in mechanically ventilated patients: a systematic review of the literature," Crit Care Med, vol. 37, no. 9, pp. 2642-7, 2009.

[13] D. De Backer, F. S. Taccone, R. Holsten, F. Ibrahimi, and J. L. Vincent, "Influence of respiratory rate on stroke volume variation in mechanically ventilated patients," Anesthesiology, vol. 110, no. 5, pp. 1092-7, 2009.

[14] A. Jerri, "Shannon sampling theorem - its various extensions and applications tutorial review." Proceedings of the IEEE, vol. 65, no. 11, p. 30, 1977.

[15] L. Faes, G. D. Pinna, A. Porta, R. Maestri, and G. Nollo, "Surrogate data analysis for assessing the significance of the coherence function," IEEE transactions on bio-medical engineering, vol. 51, no. 7, pp. 1156-66, 2004.

[16] M. Rother, H. Witte, U. Zwiener, M. Eiselt, and P. Fischer, "Cardiac aliasinga possible cause for the misinterpretation of cardiorespirographic data in neonates," Early human development, vol. 20, no. 1, pp. 1-12, 1989.

[17] H. Witte, U. Zwiener, M. Rother, and S. Glaser, "Evidence of a previously undescribed form of respiratory sinus arrhythmia (rsa)-the physiological manifestation of "cardiac aliasing"," Pflugers Archiv : European journal of physiology, vol. 412, no. 4, pp. 442-4, 1988.

[18] W. Mitzner, F. Gioia, G. G. Weinmann, J. L. Robotham, and W. Ehrlich, "Interaction between high frequency jet ventilation and cardiovascular function," Annals of biomedical engineering, vol. 15, no. 3-4, pp. 319-29, 1987.

[19] A. C. Pinchak, J. F. Hagen, D. E. Hancock, and C. Kovijanic, "Beat frequencies in high-frequency positive-pressure ventilation," Critical care medicine, vol. 12, no. 9, pp. 729-33, 1984.

[20] C. Papastamelos, H. B. Panitch, S. E. England, and J. L. Allen, "Developmental changes in chest wall compliance in infancy and early childhood," Journal of applied physiology, vol. 78, no. 1, pp. 179-84, 1995.

[21] D. De Backer, S. Heenen, M. Piagnerelli, M. Koch, and J. L. Vincent, "Pulse pressure variations to predict fluid responsiveness: influence of tidal volume," Intensive Care Med, vol. 31, no. 4, pp. 517-23, 2005. 
[22] X. Monnet, A. Bleibtreu, A. Ferre, M. Dres, R. Gharbi, C. Richard, and J. L. Teboul, "Passive leg-raising and end-expiratory occlusion tests perform better than pulse pressure variation in patients with low respiratory system compliance," Critical care medicine, vol. 40, no. 1, pp. 152-7, 2012.

[23] L. Muller, G. Louart, P. J. Bousquet, D. Candela, L. Zoric, J. E. de La Coussaye, S. Jaber, and J. Y. Lefrant, "The influence of the airway driving pressure on pulsed pressure variation as a predictor of fluid responsiveness," Intensive care medicine, vol. 36, no. 3, pp. 496-503, 2010.

[24] E. Pereira de Souza Neto, S. Grousson, F. Duflo, C. Ducreux, H. Joly, J. Convert, C. Mottolese, F. Dailler, and M. Cannesson, "Predicting fluid responsiveness in mechanically ventilated children under general anaesthesia using dynamic parameters and transthoracic echocardiography," British journal of anaesthesia, vol. 106, no. 6, pp. 856-64, 2011.

[25] X. Monnet and J. L. Teboul, "Assessment of volume responsiveness during mechanical ventilation: recent advances," Critical care, vol. 17, no. 2, p. 217, 2013.

[26] S. Heenen, D. De Backer, and J. L. Vincent, "How can the response to volume expansion in patients with spontaneous respiratory movements be predicted?" Crit Care, vol. 10, no. 4, p. R102, 2006.

[27] B. Lansdorp, J. Lemson, M. J. van Putten, A. de Keijzer, J. G. van der Hoeven, and P. Pickkers, "Dynamic indices do not predict volume responsiveness in routine clinical practice," British journal of anaesthesia, vol. 108, no. 3, pp. 395-401, 2012.

[28] X. Monnet, A. Bataille, E. Magalhaes, J. Barrois, M. Le Corre, C. Gosset, L. Guerin, C. Richard, and J. L. Teboul, "End-tidal carbon dioxide is better than arterial pressure for predicting volume responsiveness by the passive leg raising test," Intensive care medicine, vol. 39, no. 1, pp. 93-100, 2013.

[29] X. Monnet and J. L. Teboul, "Passive leg raising," Intensive Care Med, vol. 34, no. 4, pp. 659-63, 2008.

[30] B. F. Geerts, J. Maas, R. B. de Wilde, L. P. Aarts, and J. R. Jansen, “Arm occlusion pressure is a useful predictor of an increase in cardiac output after fluid loading following cardiac surgery," European journal of anaesthesiology, vol. 28, no. 11, pp. 802-6, 2011.

[31] J. J. Maas, M. R. Pinsky, L. P. Aarts, and J. R. Jansen, "Bedside assessment of total systemic vascular compliance, stressed volume, and cardiac function 
curves in intensive care unit patients," Anesthesia and analgesia, vol. 115, no. 4, pp. 880-7, 2012.

[32] V. Lukito, M. M. Djer, A. H. Pudjiadi, and Z. Munasir, "The role of passive leg raising to predict fluid responsiveness in pediatric intensive care unit patients," Pediatric critical care medicine : a journal of the Society of Critical Care Medicine and the World Federation of Pediatric Intensive and Critical Care Societies, vol. 13, no. 3, pp. e155-60, 2012. 


\section{Part II \\ Intra-thoracic pressure distribution during mechanical ventilation in health and disease}





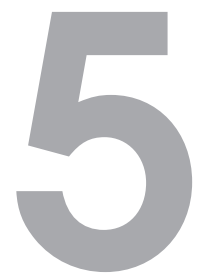

\title{
Mechanical ventilation-induced intra-thoracic pressure distribution and heart-lung interactions
}

\author{
B. Lansdorp, C. Hofhuizen, M. van Lavieren, H. van Swieten, J. Lemson, M.J.A.M. \\ van Putten, J.G. van der Hoeven and P.Pickkers. \\ Critical Care Medicine 2014 April 16 [Epub ahead of print]
}

\begin{abstract}
Objective: Mechanical ventilation causes cyclic changes in the hearts preload and afterload, thereby in fluencing the circulation. However, our understanding of the exact physiology of this cardiopulmonary interaction is limited. We aimed to thoroughly determine airway pressure distribution, how this is influenced by tidal volume (TV) and chest compliance (Cchest), and its interaction with the circulation in humans during mechanical ventilation.
\end{abstract}

Measurements and Intervention: Central venous- (CVP), airway- (Paw), pericardial- (Ppc) and pleural $(\mathrm{Ppl})$ pressure, pulse pressure-, systolic pressure- and stroke volume variations, and cardiac output were obtained in 20 mechanically ventilated patients following CABG-surgery during controlled mechanical ventilation at TVs of 4, 6, 8 and $10 \mathrm{~mL} / \mathrm{kg}$ with normal and decreased Cchest.

Main Results: With increasing TV (4-6-8-10 ml/kg), the change in intrathoracic pressures increased linearly with $0.9 \pm 0.2,0.5 \pm 0.3,0.3 \pm 0.1$ and $0.3 \pm 0.1 \mathrm{mmHg} / \mathrm{ml} / \mathrm{kg}$, for Paw, Ppl, Ppc and CVP, respectively. At $8 \mathrm{ml} / \mathrm{kg}$, a decrease in Cchest (from $0.12 \pm 0.07$ to $0.09 \pm 0.03 \mathrm{~L} / \mathrm{cmH} 2 \mathrm{O}$ ) resulted in an increase in $\mathrm{dPaw}, \mathrm{dPpl}, \mathrm{dPpc}$ and $\mathrm{dCVP}$ of $1.1 \pm 0.7,1.1 \pm 0.8,0.7 \pm 0.4$ and $0.8 \pm 0.4 \mathrm{mmHg}$, respectively. Furthermore, increased TV and decreased Cchest decreased stroke volume and increased arterial pressure variations. Transmural pressure of the superior vena cava decreased during inspiration, whereas 
the transmural pressure of the right atrium did not change.

Conclusions: Increased TV and decreased chest wall compliance both increase the change in intrathoracic pressures and the value of the dynamic indices during mechanical ventilation. Additionally, the transmural pressure of the vena cava is decreased while transmural pressure of the right atrium is not changed. 


\subsection{Introduction}

Intermittent positive airway pressure during mechanical ventilation periodically increases intra-thoracic pressure (ITP) and thereby influences the circulation. The temporary increase in airway-, pleural- and pericardial pressure (Paw, $\mathrm{Ppl}$ and $\mathrm{Ppc}$, respectively) decrease output of the right heart and, after an initial increase, also decrease output of the left heart [1]. These ventilation-induced changes, quantified by pulse pressure variation (PPV), systolic pressure variation (SPV) and stroke volume variation (SVV), can be used to predict the response to intravascular fluid administration in mechanically ventilated patients (volume responsiveness) [2,3]. The principles of heart-lung interaction and clinical usefulness of the dynamic indices to predict fluid responsiveness have been described previously [4-10]. However, to our knowledge, there is no study in which the pressure distribution is meticulously described from airway to pericardium under different conditions and related to the characteristic changes in the circulation in humans. This might also partly explain the current limitation of the clinical usefulness of the dynamic indices to predict volume responsiveness in clinical practice [11]. To improve our knowledge about the underlying physiology of heart-lung interaction, the primary aim of the present study was to show to what extent the airway pressure is distributed to the pleural-, pericardial- and central venous pressure (CVP) and how this distribution is influenced by changes in tidal volume and chest wall compliance. The secondary objective was to determine how this pressure distribution influences circulation by changing transmural pressures of the right atrium (and thereby right cardiac pre- and afterload) and dynamic indices and how this is related to fluid responsiveness.

\subsection{Materials and Methods}

With the approval of the institutional review board and following written informed consent, 20 patients were studied following elective coronary artery bypass grafting (CABG). Exclusion criteria were any cardiac arrhythmias, hemodynamic instability (a change in heart rate or blood pressure $>20 \%)$, recent myocardial infarction $(<2$ months), preoperative inotropic or intra-aortic balloon pump support, non-elective surgery and concomitant pulmonary, endocrine, metabolic or neurologic disease. Prior to surgery, each patient received a central venous (internal jugular vein) and arterial (radial artery) catheter. During surgery, an air-filled 5-F balloon catheter (12X20mm Tyshak II, NuMed, Canada) was placed in the pleural space (juxtacardiac position) and another one in the pericardial space (at the lateral aspect of the right atrium) and a 5-F thermistor-tipped arterial PiCCO ${ }^{\circledR}$ catheter (Pulsion Medical Systems, Munich, Germany) was inserted into the femoral artery. 


\subsubsection{Monitoring}

On arrival on the ICU, patients were mechanically ventilated (volume-controlled) with the Servo 300 (Macquet, Rasstat, Germany) and Paw and TV were monitored with a dedicated respiratory monitor (NICO, Philips respironics, Amsterdam, the Netherlands). Arterial blood pressure (ABP), CVP and EKG were monitored using a HP monitor (Merlin M1046A, Hewlett Packard, CA, USA). Cardiac output and stroke volume index (SVI) were monitored by a PiCCO monitoring system (PiCCO 2.0, Pulsion Medical Systems, Munich, Germany) through thermodilution and calibrated pulse contour analysis. The pericardial and pleural catheter were filled with $0.8 \mathrm{ml}$ of air. This amount of air was determined during in vitro experiments prior to the study as the smallest amount of air (in order to minimize possible distortion of the pericardium) that creates a lumen inside the balloon that adjusts to peripheral pressure (positive or negative) and was much smaller than the balloons unstressed volume of $7 \mathrm{ml}$. All parameters were recorded on with a sample rate of $200 \mathrm{~Hz}$. All pressures are expressed in $\mathrm{mmHg}$.

Transmural pressure $(\mathrm{Ptm})$ of the superior vena cava (PtmSVC) was defined as CVPPpl, and Ptm of the right atrium (PtmRA) was calculated as CVPPpc. The PPV, SPV and SVV were defined as the mean value of the relative difference in pulse pressure, systolic pressure and stroke volume over one breath, averaged over a period of 5 breaths(11).

\subsubsection{Design}

After hemodynamic stability was reached (heart rate or mean arterial pressure fluctuations $<10 \%$ ) and calibration of the PiCCO, patients were ventilated with 4 increasing values of TV: $4-6-8-10 \mathrm{ml} / \mathrm{kg}$ ideal body weight (starting at $4 \mathrm{ml} / \mathrm{kg}$ ). This was repeated during decreased chest wall compliance, by placing an elastic band around the patients thorax, aimed to decrease total respiratory system compliance with $25 \%$. During these 3-minute-periods, minute volume was kept constant by adjusting respiratory rate. The change of the transmural pressure variation over the different levels of tidal volume was related to the value of the pulse pressure variation (as a measure of fluid responsiveness).

\subsubsection{Statistical analysis}

Differences in the effect of changes in TV and chest wall compliance were compared with repeated measures two-way analysis of variance (ANOVA) since data was normally distributed, including Bonferroni tests for subgroups. Paired Students t-tests were used to compare the means of two groups. P-values $<0.05$ were considered statistically significant. Data is presented as mean \pm standard deviation and statistical 

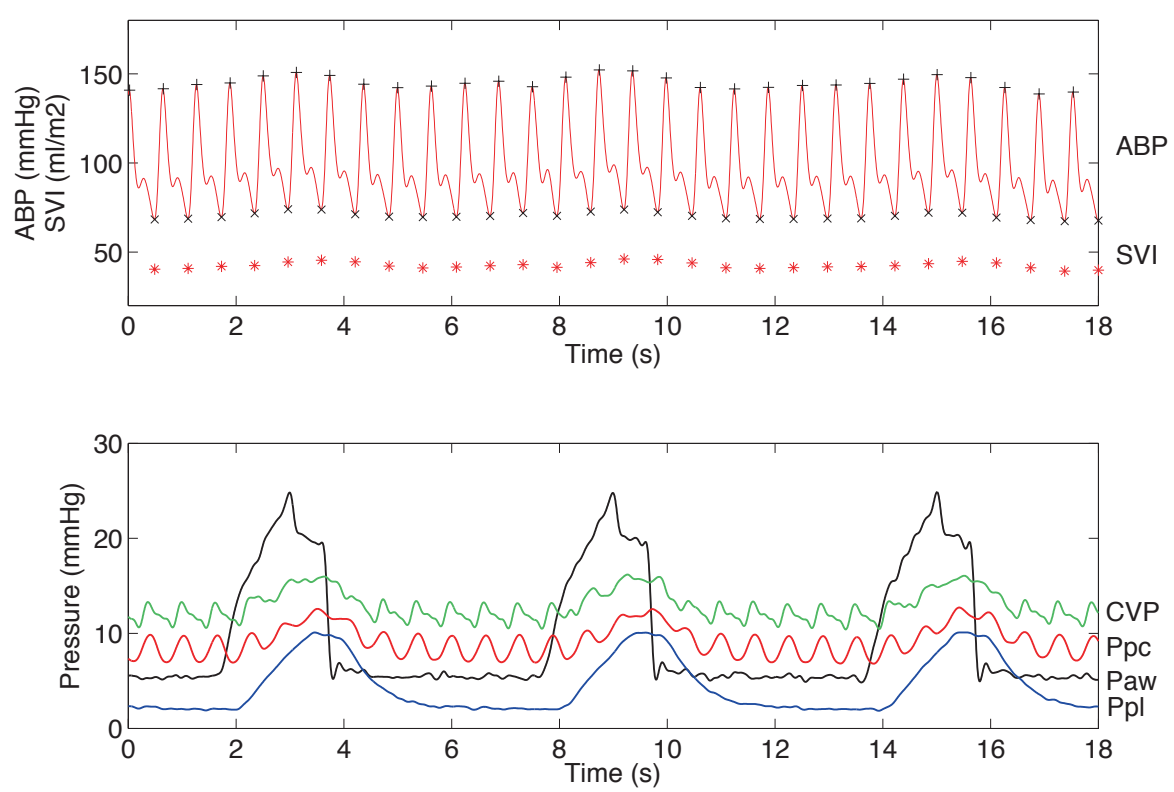

Figure 1: Representative example of the simultaneous recording of the arterial blood pressure $(A B P)$ and stroke volume index (SVI) (upper part) and central venous pressure $(C V P)$, airway pressure (Paw), juxtacardiac pleural pressure $(P p l)$ and pericardial pressure $(P p c)$ during mechanical ventilation at $8 \mathrm{ml} / \mathrm{kg}$ during volume controlled ventilation.

analysis was performed using SPSS 19 for Mac (SPSS Inc., Chicago, Ill, USA) and Prism 5 for Mac (Graphpad Software Inc, La Jolla, CA, USA).

\subsection{Results}

Patient characteristics and baseline hemodynamic and ventilatory parameters on ICU admission are presented in Table 1. A representative example of the simultaneous recordings of ABP, SVI, Paw, Ppl, Ppc, and CVP is shown in Figure 1. Figure 2 presents pressure-volume loops during consecutive breaths of Paw, Ppl and Ppc with the four different tidal volumes. With increasing tidal volumes (and adjusted respiratory rates, resulting in a mean heart rate to respiratory rate of $3.9 \pm 0.6$ at $4 \mathrm{ml} / \mathrm{kg}$ ), the change in intrathoracic pressures increased significantly $(\mathrm{p}<0.01)$ and linearly with $0.9 \pm 0.2,0.5 \pm 0.3,0.3 \pm 0.1$ and $0.3 \pm 0.1 \mathrm{mmHg} / \mathrm{ml} / \mathrm{kg}$, for Paw, Ppl, Ppc and 

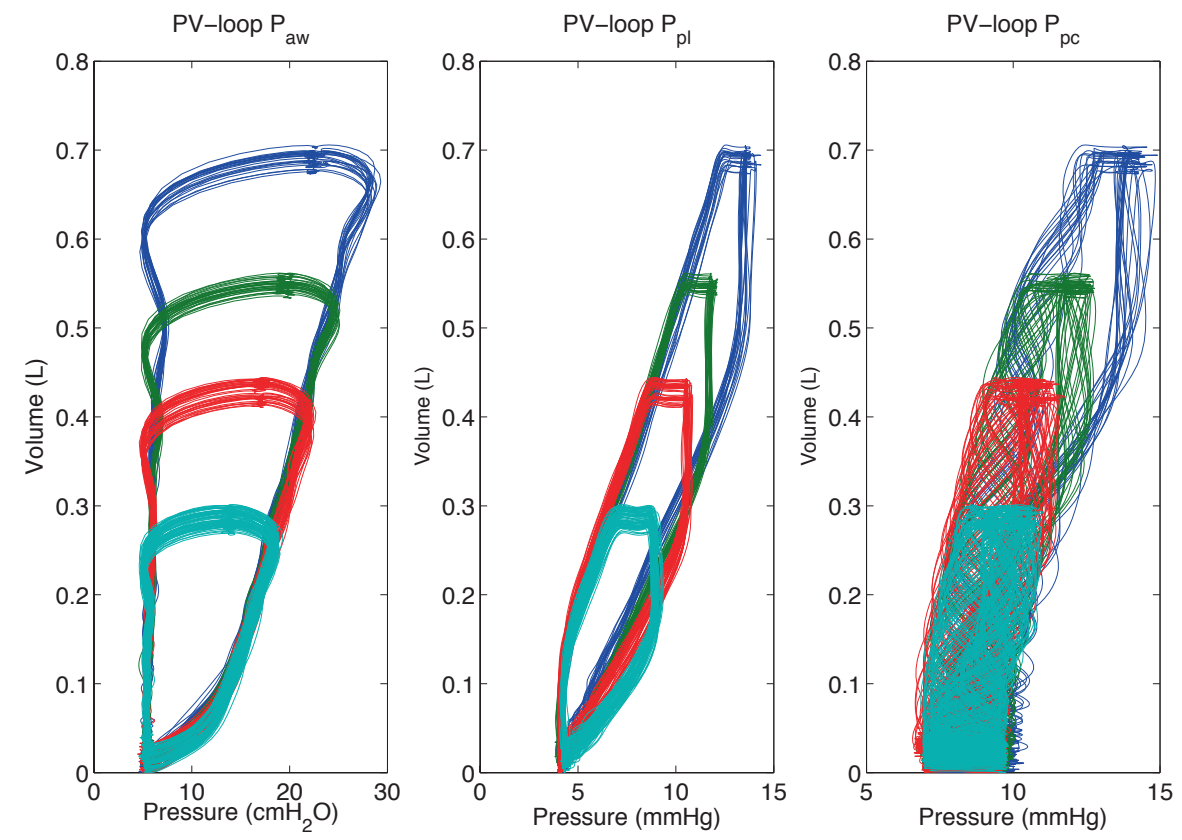

Figure 2: Pressure-Volume loops of the airway pressure (Paw, part A), juxtacardiac pleural pressure (Ppl, part B) and pericardial pressure (Ppc, part $C)$ during multiple consecutive mechanically ventilated breaths with 4, 6, 8 and $10 \mathrm{ml} / \mathrm{kg}$. In the pericardial pressure (part C), also the contractions of the heart are visible. 
CVP, respectively (Figure 3). Elastic banding around the thorax significantly decreased chest wall compliance with $0.03 \pm 0.01 \mathrm{~L} / \mathrm{cmH} 2 \mathrm{O}(23 \pm 12 \%$, from 0.12 to $0.09 \mathrm{~L} / \mathrm{cmH} 2 \mathrm{O}$ ), increased end-expiratory pleural-, pericardial- and central venous pressure (from $5.1 \pm 1.7 \mathrm{mmHg}$ to $6.3 \pm 1.2 \mathrm{mmHg}, 7.0 \pm 2.3 \mathrm{mmHg}$ to $8.9 \pm 2.1 \mathrm{mmHg}$ and $10 \pm 2.7 \mathrm{mmHg}$ to $11.6 \pm 2.6 \mathrm{mmHg}$, respectively, all $\mathrm{p}<0.01)$ and decreased stroke volume index (from $41.5 \pm 5.9$ to $39.5 \pm 7.1 \mathrm{ml} / \mathrm{m} 2, \mathrm{p}<0.01$ ) at tidal volume of $8 \mathrm{ml} / \mathrm{kg}$. At the different tidal volumes, the decreased chest wall compliance resulted in a $13 \pm 7 \%, 40 \pm 21 \%, 35 \pm 16 \%$ and $26 \pm 12 \%$ increase in dPaw, dPpl, dPpc and dCVP, respectively (all $\mathrm{p}<0.01$, Figure 3 ). At a tidal volume of $8 \mathrm{ml} / \mathrm{kg}$, this meant an increase in dPaw, dPpl, dPpc and dCVP of $1.1 \pm 0.7 \mathrm{mmHg}, 1.1 \pm 0.8 \mathrm{mmHg}, 0.7 \pm 0.4 \mathrm{mmHg}$ and $0.8 \pm 0.4 \mathrm{mmHg}$ (Table 2).

Table 1: Patients characteristics and baseline haemodynamic and respiratory variables, presented as mean (range), mean (SD) or number.

\begin{tabular}{||l|l||}
\hline \multicolumn{2}{||c||}{ patient characteristics } \\
Patient (\#) & 20 \\
Male/Female (\#) & $17 / 3$ \\
Age $(\mathrm{yr})$ & $66 \pm 7.9$ \\
Weight $(\mathrm{kg})$ & $86.6 \pm 16.2$ \\
\hline \multicolumn{2}{|c||}{ Hemodynamic parameters on ICU admission } \\
\hline Heart rate $\left(\right.$ beats $\left.\cdot \mathrm{min}^{-1}\right)$ & $84.1 \pm 19.5$ \\
Mean arterial pressure $(\mathrm{mmHg})$ & $66.5 \pm 8.4$ \\
Central venous pressure $(\mathrm{mmHg})$ & $11.2 \pm 3.6$ \\
Cardiac output $\left(\mathrm{L} \cdot \mathrm{min}^{-1}\right)$ & $6.1 \pm 1.1$ \\
\hline \multicolumn{2}{|c|}{ Respiratory parameters on ICU admission } \\
\hline Tidal volume $\left(\mathrm{ml}^{\prime} \cdot \mathrm{kg}_{1} \mathrm{IBW}\right)$ & $6.9 \pm 0.8$ \\
Respiratory rate $\left(\mathrm{breaths}^{\mathrm{m}} \cdot \mathrm{min}^{-1}\right)$ & $12.6 \pm 1.2$ \\
Positive end-expiratory pressure $\left(\mathrm{cmH}_{2} \mathrm{O}\right)$ & $6.2 \pm 1.8$ \\
Plateau pressure $\left(\mathrm{cm} \mathrm{H}_{2} \mathrm{O}\right)$ & $19.0 \pm 4.4$ \\
\hline \multicolumn{2}{|c|}{}
\end{tabular}




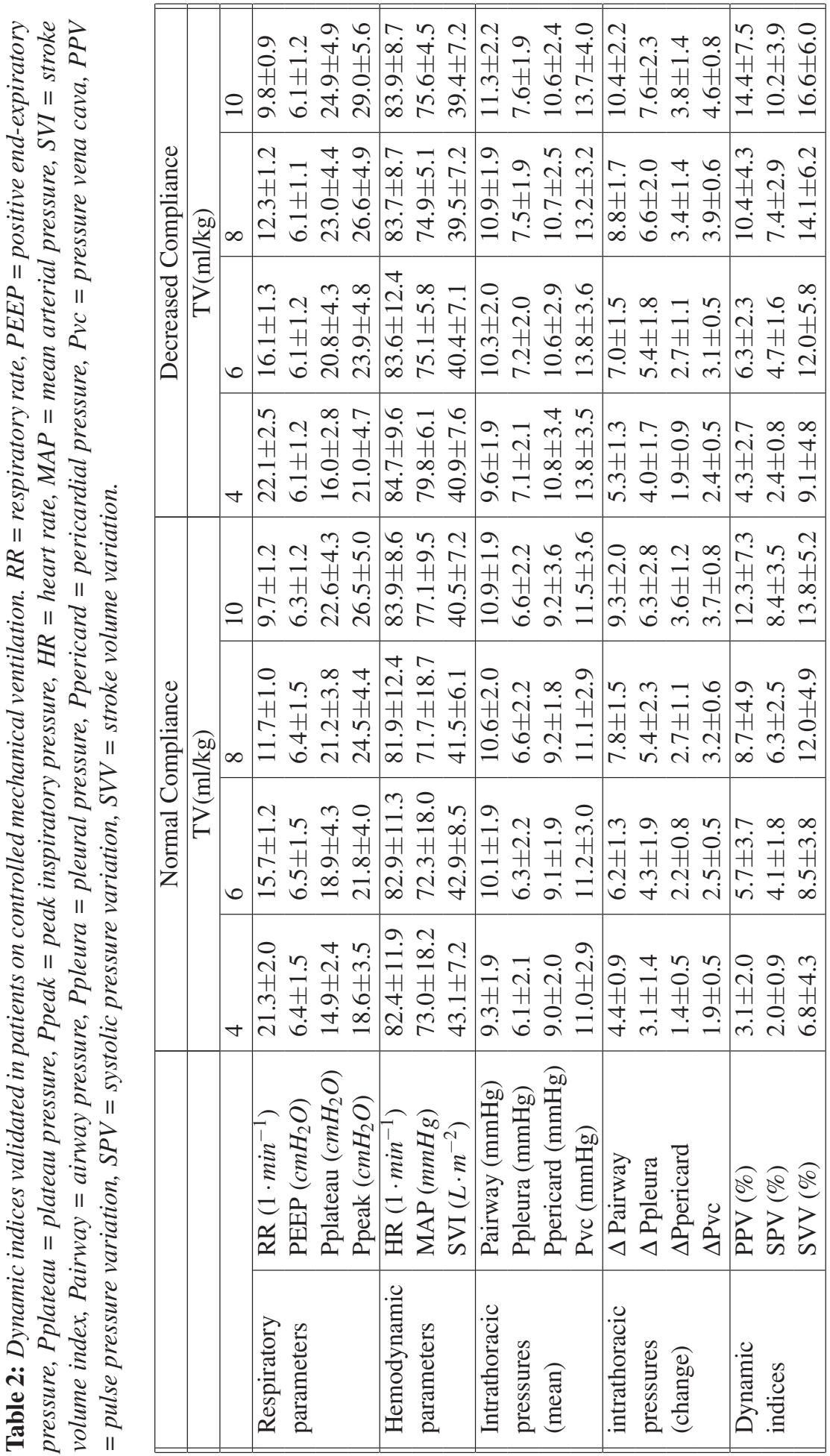


The percentage of airway pressure that was transmitted to the juxtacardiac pleura, pericardium and vena cava was $70 \pm 27,37 \pm 17$ and $43 \pm 11 \%$, respectively (Figure $4 a)$. These percentages did not change significantly with different tidal volumes. However, a small but significant $(\mathrm{p}<0.01)$ larger proportion of airway pressure was transmitted when chest wall compliance was reduced $(77 \pm 21,39 \pm 19$ and $46 \pm 11 \%$, respectively for juxtacardiac pleura, pericardium and vena cava). The different extent to which the Paw is transmitted to the Ppl, Ppc and CVP resulted in a significant decrease in the transmural pressure of the superior vena cava $(\mathrm{p}<0.01)$, while transmural pressure of the right atrium remained unchanged $(\mathrm{p}>0.1)$ during positive pressure ventilation (Figure $4 \mathrm{~b}$ ). The alternating intrathoracic and transmural pressures during mechanical ventilation also caused the SVI to change periodically. SVI significantly decreased with increasing TV (from $43 \pm 6 \mathrm{ml} / \mathrm{m} 2$ at $4 \mathrm{ml} / \mathrm{kg}$ to $39 \pm 6 \mathrm{ml} / \mathrm{m} 2$ at $10 \mathrm{ml} / \mathrm{kg}, \mathrm{p}<0.01)$ and was significantly lower with decreased chest wall compliance (mean difference of $-2.5 \pm 3.3 \mathrm{ml} / \mathrm{m} 2$ over all tidal volumes, $\mathrm{p}<0.01$ ). The cyclic changes in SVI during MV resulted in arterial pressure variations (PPV, SPV and SVV) that were more pronounced at higher levels of TV and lower chest wall compliance (Figure 5). To determine whether the underlying mechanism of heartlung interaction is related to volume status, the change of the transmural pressure variation over the different levels of tidal volume was related to the value of the pulse pressure variation. Although the PtmRA of the overall population was unchanged during mechanical ventilation, two distinct patterns became apparent. While some patients consistently showed an increase, others showed a decrease in PtmRA (Figure 6), which became more pronounced with increasing tidal volumes. Figure 7 illustrates the quantification of this change in transmural pressure variation as a result of the increasing tidal volume using the slope of the trend line. This slope was positive for patients in which the change in transmural pressure increased, suggesting that the influence of the afterload is becoming more pronounced with increasing tidal volume.

\subsection{Discussion}

The present study demonstrates to what extent positive pressure ventilation influences intrathoracic pressures and thereby transmural pressures and dynamic cardiovascular indices and how this is influenced by different levels of tidal volume and chest wall compliance in ventilated ICU patients. Our results show that the ratio of airway pressure that is distributed to the pleura (about 2/3rd) is almost double the percentage distributed to the pericardium and vena cava (about $1 / 3$ rd), that tidal volume in a clinically relevant range does not influence these percentages, and that a lower chest wall compliance results in a larger portion of airway pressure that is transmitted to the Ppl, Ppc and CVP. Our data also show that increased variations in intrathoracic 
Change in airway pressure

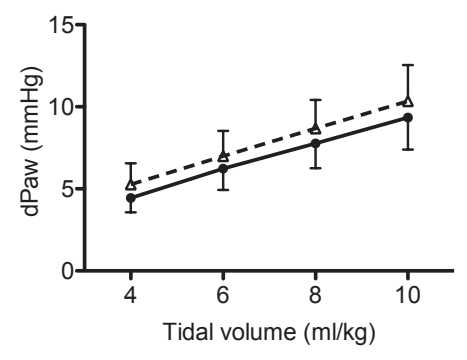

Change in pericardial pressure

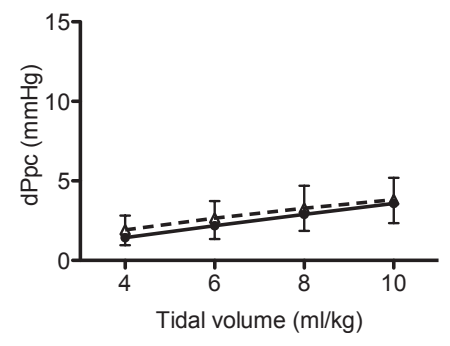

— normal compliance
Change in pleural pressure

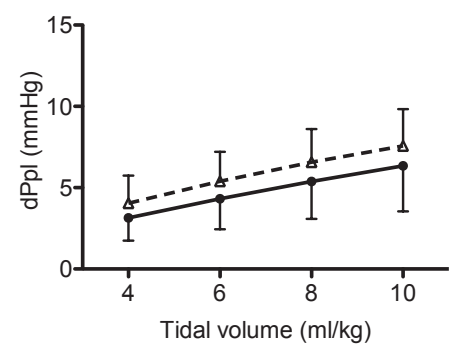

Change in central venous pressure

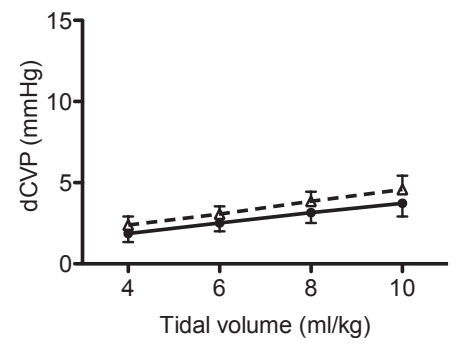

- - decreased compliance

Figure 3: Change (in $\mathrm{mmHg}$ ) in airway pressure (part A), juxtacardiac pleural pressure (part $B)$, pericardial (part $C$ ) and central venous pressure (part D) due to the different tidal volumes $(4,6,8$ and $10 \mathrm{ml} / \mathrm{kg}$ ) at baseline (lower line) and with decreased chest wall compliance using an elastic band. Increased tidal volume and decreased chest wall compliance both result in increased portions of transmitted airway pressures. 


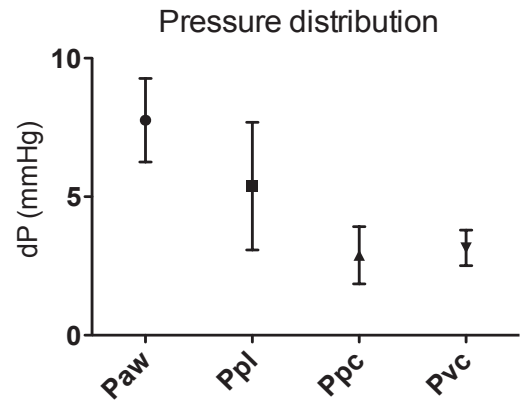

pressure site

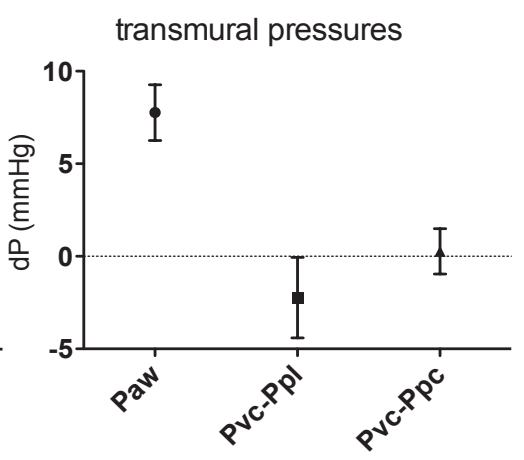

pressure site

Figure 4: Change in airway pressure (Paw), juxtacardiac pleural pressure (Ppl), pericardial pressure $(P p c)$ and pressure in the vena cava $(P v c)($ part $A)$ and the change in transmural pressure of the superior vena cava (Pvc minus Ppl) and right atrium (Pvc-Ppc) (part B) during mechanical ventilation with $8 \mathrm{ml} / \mathrm{kg}$. 

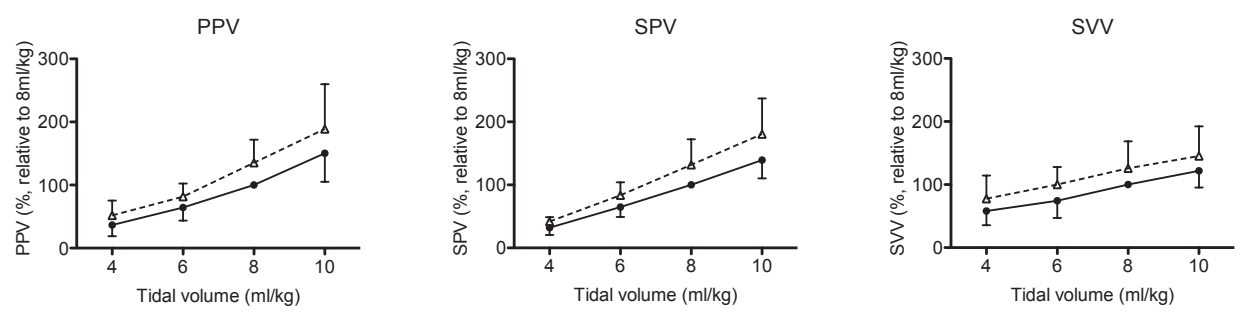

- normal compliance

-- decreased compliance

Figure 5: Change of dynamic indices as a result of increasing tidal volume and decreasing chest compliance as a percentage of the value at $8 \mathrm{ml} / \mathrm{kg}$ with normal compliance. Increased tidal volume and decreased chest wall compliance both result in increased values of the dynamic indices. $P P V=$ pulse pressure variation, $S P V=$ systolic pressure variation, $S V V=$ stroke volume variation. 

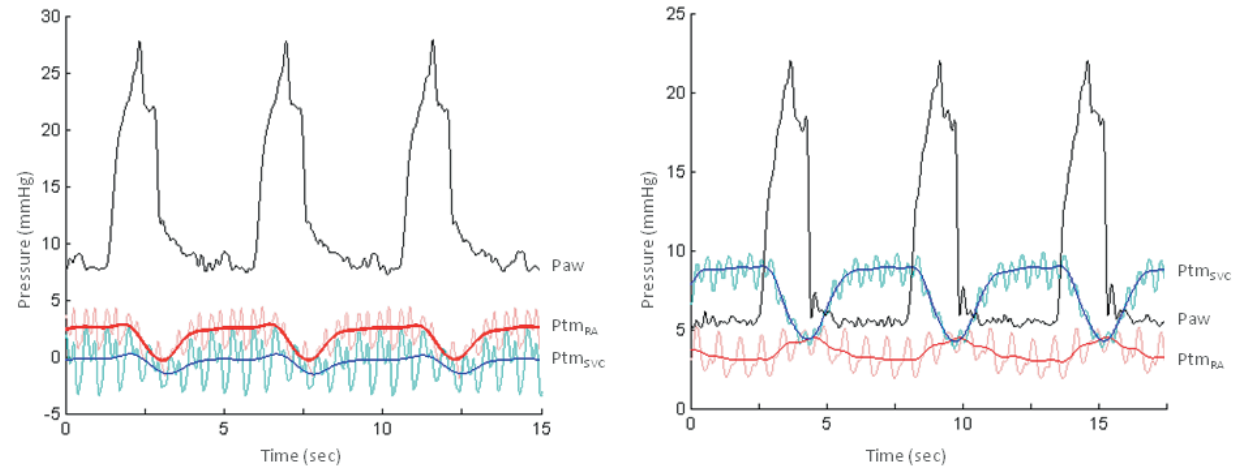

Figure 6: Influence of increased airway pressure (black line) on transmural pressure of the right atrium (red line) and superior vena cava (blue line). Both pressures are displayed with and without filtering of the heartbeat. In both patients, the transmural pressure of the superior vena cava (SVC) decreases, just like the transmural pressure of the right atrium in part A of the figure, whereas in part $B$, the transmural pressure of the right atrium (RA) increases. 


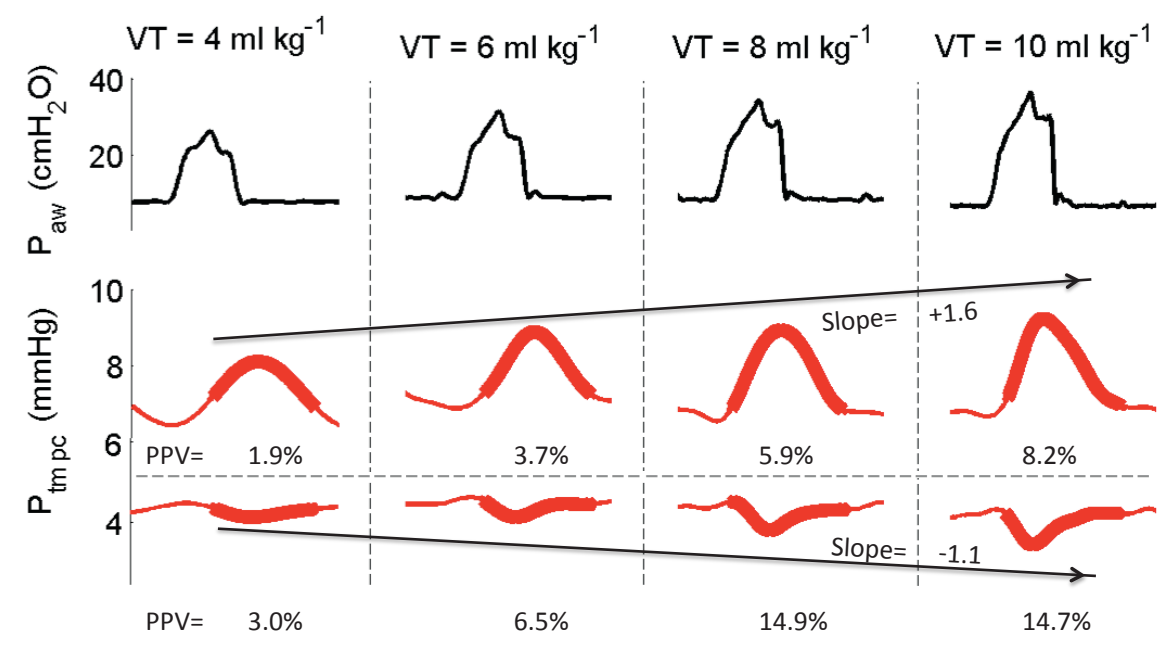

Figure 7: illustration of the quantification of the change of the transmural pressure variations as a result of increasing tidal volumes. The upper part shows the airway pressure during the 4 different levels of tidal volume. The middle and the lower part illustrate the (change in) transmural pressure of two different patients during these levels of tidal volume (of a patient with low and high dynamic indices, respectively). A positive slope (illustrated in the middle part which reflects the patients with low dynamic indices, being nonpreloaddependent) shows that the change of the transmural pressure of the right atrium increases (becomes more positive), indicating that the influence of the afterload is becoming more pronounced with increasing tidal volumes. On the other hand, the patient with high dynamic indices (being preload-dependent) shows a negative slope, indicating that the preload is being decreased more than the afterload is being increased. VT = Tidal volume, Paw = Airway Pressure, $P$ tmPC $=$ transmural pressure of the pericard, $P P V=$ Pulse Pressure Variation. 
pressures also result in more pronounced variations in stroke volume and thereby higher values of the dynamic indices (PPV, SPV and SVV). Additionally, we showed that the transmural pressure of the superior vena cava decreases during inspiration, whereas the transmural pressure of the right atrium does not change. Tidal volume is more relevant than Paw, as lung compliance influences the transmission of Paw to the other intrathoracic cavities $[12,13]$. Our results confirm this finding in ventilated humans as tidal volume almost linearly increases intrathoracic pressures and dynamic indices. Besides the effect of lung compliance on the transmission of Paw, also chest wall compliance influences the effect of Paw on intrathoracic pressures. In accordance, we show that in humans, reduced chest wall compliance increases airway pressure transmission. While previous studies described the influence on ventricular performance [5, 14], and others focused on, e.g., left ventricular filling pressures $[6,15]$, our study simultaneously measured Paw, Ppl, Ppc, CVP (and thereby ventilatory induced changes in right-sided transmural pressures). In contradiction to the general assumption that the increased intrathoracic pressure is transmitted to the right atrium and thereby decreases the pressure gradient for venous return [16-18], we found no difference in transmural pressure of the right atrium during incremental tidal volumes. This result is in accordance with the observation that transmural pressure of the right atrium does not change during increasing levels of PEEP [7], and also shown in a prior study that found that the increase in right atrial pressure was fully explained by an increase in pericardial pressure [19]. As the right atrial compliance is high, right atrial filling volume remains unchanged, which is also in line with the results of other studies [20,21]. The mechanism of action may be twofold. First, the associated increase in abdominal pressure during inspiration (partly) prevents the pressure gradient for venous return to decrease. However, while the transmural pressure of the right atrium remains constant, the transmural pressure of the vena cava decreases during inspiration. This is caused by the different extent to which the Paw is transmitted to the Ppl and Ppc during each ventilatory cycle. Apparently, there is a decrease in venous return, but this effect is neutralised by the second mechanism, which is the increase in afterload. Since the increase of transpulmonary pressure increases right ventricular afterload during lung inflation [22], this results in an increase in transmural pressure of the right atrium. Indeed, we found that the the effect of a reduced venous return on transmural pressure of the right atrium is neutralised by a raised RV afterload. This finding is in accordance with earlier published data, that showed that an inspiratory hold first results in an increase in right ventricular output, followed by a decrease [23]. A more detailed analysis of our data shows two distinct patterns within our patient population. While some patients consistently showed an increase, others showed a decrease in PtmRA, which became more pronounced with increasing tidal volumes. We hypothesize that this change of the transmural pressure variation is related to the patients volume status and that in patients with lower 
dynamic indices the transmural pressure variation of the right atrium increases with increasing tidal volumes, suggesting a more pronounced role of the right ventricural afterload in nonpreload-dependent patients. However, no significant difference was found in the change of the transmural pressure variation of the RA between the patients with a high compared to the patients with a low pulse pressure variation. Besides the described influences of mechanical ventilation on the right side of the heart, changes in intrathoracic pressures also influences the pre- and afterload of the left ventricle. The increase in pleural pressure decreases left ventricular afterload by decreasing the pressure gradient between the left ventricle and the extrathoracic systemic circulation. Furthermore, the increase in transpulmonary pressure increases left ventricular preload [1]. We also demonstrate the influence of tidal volume on intrathoracic pressures and dynamic indices. Since the suggested thresholds $(12,7$ and $12 \%$ for PPV, SPV and SVV, respectively) $[3,24]$ are based on TV $>8 \mathrm{ml} / \mathrm{kg}$, while in clinical practice it is preferred to ventilate with smaller tidal volumes, we advocate that these thresholds should be adjusted for the actual applied tidal volume. While this has been suggested earlier [25], our study provides physiological and quantitative data for this correction in humans. Based on our results, published threshold values for the prediction of fluid responsiveness should be decreased with 19, 18 and $11 \%$ for PPV, SPV and SVV, respectively, for each $\mathrm{ml} / \mathrm{kg}$ tidal volume when tidal volume is lower (and increased when tidal volume is higher) than $8 \mathrm{ml} / \mathrm{kg}$. Furthermore, correction of the threshold for a change in chest wall compliance should be done with $23,-18$ and $-11 \%$ for each $10 \mathrm{ml} / \mathrm{cmH} 2 \mathrm{O}$ change in chest wall compliance for PPV, SPV and SVV, respectively. However, regarding the correction for chest wall compliance, it should be noted that in our study, elastic binding of the thorax increased end-expiratory intrathoracic pressures. Since elastic binding also influenced stroke volume index, this might also increase preload-dependency of the patient. Limitations Several limitations of our study need to be addressed. First, the signal/noise ratio of pericardial pressure was relatively low, because of both the relatively small changes in pressure and movements of the beating heart. To prevent potential distortion of the pericardial pressure due the balloon used to measure Ppc, we used air filled balloons with the smallest amount of air that gave similar pressure in the balloon as the surrounding pressure during ex vivo measurements. This method and the presented results are comparable to the results of other animal and human studies [7, 26-30], which confirms its reliability. Second, we used the PiCCO device to monitor cardiac output and stroke volume index. The pulse contour analysis used in the PiCCO device was calibrated by transpulmonary thermodilution; however, errors in accuracy and precision may be considerable [31,32]. Third, our patient population may not be similar to other ventilated ICU patients. Our patients underwent cardiac surgery including a mediastinotomy and pericardiotomy. Although the pericardium and thorax were closed before the start of the measurements, ventricular interde- 
pendence caused by pericardial volume restraint may have been minimized in our patients [33]. Furthermore, our patients had no pulmonary complications resulting in impaired lung compliance. Therefore, conclusions drawn from this homogeneous patient population to other ventilated ICU patients should be done with caution.

\subsection{Conclusion}

Increased tidal volume and decreased chest wall compliance both increase the change in intrathoracic pressures during mechanical ventilation and result in more pronounced variations in stroke volume and thereby higher values of the dynamic indices. We show that about 2/3th of the airway pressure is transmitted to the pleura and $1 / 3$ th to the pericardium and superior vena cava, resulting in a decrease of transmural pressure of the superior Vena Cava and an unchanged transmural pressure of the right atrium.

\section{References}

[1] F. Michard, "Changes in arterial pressure during mechanical ventilation," Anesthesiology, vol. 103, no. 2, pp. 419-28, 2005.

[2] S. Magder, "Clinical usefulness of respiratory variations in arterial pressure," Am J Respir Crit Care Med, vol. 169, no. 2, pp. 151-5, 2004.

[3] P. E. Marik, R. Cavallazzi, T. Vasu, and A. Hirani, "Dynamic changes in arterial waveform derived variables and fluid responsiveness in mechanically ventilated patients: a systematic review of the literature," Crit Care Med, vol. 37, no. 9, pp. 2642-7, 2009.

[4] M. R. Pinsky, "The hemodynamic consequences of mechanical ventilation: an evolving story," Intensive care medicine, vol. 23, no. 5, pp. 493-503, 1997.

[5] A. J. Buda, M. R. Pinsky, J. Ingels, N. B., n. Daughters, G. T., E. B. Stinson, and E. L. Alderman, "Effect of intrathoracic pressure on left ventricular performance," The New England journal of medicine, vol. 301, no. 9, pp. 453-9, 1979.

[6] M. Pinsky, J. L. Vincent, and J. M. De Smet, "Estimating left ventricular filling pressure during positive end-expiratory pressure in humans," The American review of respiratory disease, vol. 143, no. 1, pp. 25-31, 1991.

[7] M. R. Pinsky, J. M. Desmet, and J. L. Vincent, "Effect of positive end-expiratory pressure on right ventricular function in humans," The American review of respiratory disease, vol. 146, no. 3, pp. 681-7, 1992. 
[8] A. Y. Denault, T. A. Gasior, r. Gorcsan, J., W. A. Mandarino, L. G. Deneault, and M. R. Pinsky, "Determinants of aortic pressure variation during positivepressure ventilation in man," Chest, vol. 116, no. 1, pp. 176-86, 1999.

[9] J. A. Romand, W. Shi, and M. R. Pinsky, "Cardiopulmonary effects of positive pressure ventilation during acute lung injury," Chest, vol. 108, no. 4, pp. 10418, 1995.

[10] P. C. van den Berg and M. R. Pinsky, "Systems approach to heart-lung interactions," The Netherlands journal of medicine, vol. 57, no. 3, pp. 113-31, 2000.

[11] B. Lansdorp, J. Lemson, M. J. van Putten, A. de Keijzer, J. G. van der Hoeven, and P. Pickkers, "Dynamic indices do not predict volume responsiveness in routine clinical practice," British journal of anaesthesia, vol. 108, no. 3, pp. 395-401, 2012.

[12] F. Jardin, B. Genevray, D. Brun-Ney, and J. P. Bourdarias, "Influence of lung and chest wall compliances on transmission of airway pressure to the pleural space in critically ill patients," Chest, vol. 88, no. 5, pp. 653-8, 1985.

[13] J. C. Chapin, J. B. Downs, M. E. Douglas, E. J. Murphy, and B. C. Ruiz, "Lung expansion, airway pressure transmission, and positive end-expiratory pressure," Archives of surgery, vol. 114, no. 10, pp. 1193-7, 1979.

[14] r. Gorcsan, J., J. A. Romand, W. A. Mandarino, L. G. Deneault, and M. R. Pinsky, "Assessment of left ventricular performance by on-line pressure-area relations using echocardiographic automated border detection," Journal of the American College of Cardiology, vol. 23, no. 1, pp. 242-52, 1994.

[15] M. R. Pinsky and J. Guimond, "The effects of positive end-expiratory pressure on heart-lung interactions," Journal of critical care, vol. 6, no. 1, p. 10, 1991.

[16] A. C. Guyton, A. W. Lindsey, B. Abernathy, and T. Richardson, "Venous return at various right atrial pressures and the normal venous return curve," The American journal of physiology, vol. 189, no. 3, pp. 609-15, 1957.

[17] J. Qvist, H. Pontoppidan, R. S. Wilson, E. Lowenstein, and M. B. Laver, "Hemodynamic responses to mechanical ventilation with peep: the effect of hypervolemia," Anesthesiology, vol. 42, no. 1, pp. 45-55, 1975.

[18] B. C. Morgan, W. E. Martin, T. F. Hornbein, E. W. Crawford, and W. G. Guntheroth, "Hemodynamic effects of intermittent positive pressure respiration," Anesthesiology, vol. 27, no. 5, pp. 584-90, 1966. 
[19] J. V. Tyberg, G. C. Taichman, E. R. Smith, N. W. Douglas, O. A. Smiseth, and W. J. Keon, "The relationship between pericardial pressure and right atrial pressure: an intraoperative study," Circulation, vol. 73, no. 3, pp. 428-32, 1986.

[20] P. C. van den Berg, J. R. Jansen, and M. R. Pinsky, "Effect of positive pressure on venous return in volume-loaded cardiac surgical patients," Journal of applied physiology, vol. 92, no. 3, pp. 1223-31, 2002.

[21] A. Vieillard-Baron, Y. Loubieres, J. M. Schmitt, B. Page, O. Dubourg, and F. Jardin, "Cyclic changes in right ventricular output impedance during mechanical ventilation," Journal of applied physiology, vol. 87, no. 5, pp. 1644-50, 1999.

[22] F. Jardin, G. Delorme, A. Hardy, B. Auvert, A. Beauchet, and J. P. Bourdarias, "Reevaluation of hemodynamic consequences of positive pressure ventilation: emphasis on cyclic right ventricular afterloading by mechanical lung inflation," Anesthesiology, vol. 72, no. 6, pp. 966-70, 1990.

[23] P. C. van den Berg, C. A. Grimbergen, J. A. Spaan, and M. R. Pinsky, "Positive pressure inspiration differentially affects right and left ventricular outputs in postoperative cardiac surgery patients," Journal of critical care, vol. 12, no. 2, pp. 56-65, 1997.

[24] F. Michard, S. Boussat, D. Chemla, N. Anguel, A. Mercat, Y. Lecarpentier, C. Richard, M. R. Pinsky, and J. L. Teboul, "Relation between respiratory changes in arterial pulse pressure and fluid responsiveness in septic patients with acute circulatory failure," Am J Respir Crit Care Med, vol. 162, no. 1, pp. 134-8, 2000.

[25] S. T. Vistisen, J. Koefoed-Nielsen, and A. Larsson, "Should dynamic parameters for prediction of fluid responsiveness be indexed to the tidal volume?" Acta Anaesthesiol Scand, 2009.

[26] M. Hadian, D. A. Severyn, and M. R. Pinsky, "The effects of vasoactive drugs on pulse pressure and stroke volume variation in postoperative ventilated patients," Journal of critical care, vol. 26, no. 3, pp. 328 e1-8, 2011.

[27] D. Mann, W. Lew, E. Ban-Hayashi, R. Shabetai, L. Waldman, and M. M. LeWinter, "In vivo mechanical behavior of canine pericardium," The American journal of physiology, vol. 251, no. 2 Pt 2, pp. H349-56, 1986.

[28] B. K. Slinker, R. V. Ditchey, S. P. Bell, and M. M. LeWinter, "Right heart pressure does not equal pericardial pressure in the potassium chloride-arrested canine heart in situ," Circulation, vol. 76, no. 2, pp. 357-62, 1987. 
[29] O. A. Smiseth, M. A. Frais, I. Kingma, E. R. Smith, and J. V. Tyberg, "Assessment of pericardial constraint in dogs," Circulation, vol. 71, no. 1, pp. 158-64, 1985.

[30] D. R. Hamilton, G. Devries, and J. V. Tyberg, "Static and dynamic operating characteristics of a pericardial balloon," Journal of applied physiology, vol. 90, no. 4, pp. 1481-8, 2001.

[31] E. E. de Waal, F. Wappler, and W. F. Buhre, "Cardiac output monitoring," Curr Opin Anaesthesiol, vol. 22, no. 1, pp. 71-7, 2009.

[32] M. Hadian, H. K. Kim, D. A. Severyn, and M. R. Pinsky, "Cross-comparison of cardiac output trending accuracy of lidco, picco, flotrac and pulmonary artery catheters," Critical care (London, England), vol. 14, no. 6, p. R212, 2010.

[33] S. A. Glantz, G. A. Misbach, W. Y. Moores, D. G. Mathey, J. Lekven, D. F. Stowe, W. W. Parmley, and J. V. Tyberg, "The pericardium substantially affects the left ventricular diastolic pressure-volume relationship in the dog," Circulation research, vol. 42, no. 3, pp. 433-41, 1978. 


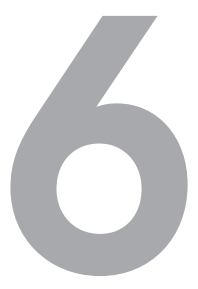

\title{
Intra-thoracic pressure distribution during mechanical ventilation in a newborn animal model: influence of tidal volume and progressive ARDS
}

\author{
B. Lansdorp, A. Nusmeier, S. Vranken, J. van der Hoeven, W.P de Boode and J. \\ Lemson \\ In preparation for Journal of Applied Physiology
}

\footnotetext{
Background. Mechanical ventilation causes cyclic changes in the hearts preload and afterload, thereby influencing the circulation. However, our understanding of the exact physiology of this cardiopulmonary interaction in children and in conditions of decreased lung compliance is limited. We aimed to show to what extent the airway pressure is distributed to the central venous pressure (CVP), how this influences mechanically ventilated induced preload indicators, and how this distribution is influenced by changes in tidal volume (TV) and lung compliance in a newborn animal.

Methods. In 11 newborn lambs, airway pressure (Paw), central venous pressure (CVP) and cardiac output were obtained during controlled mechanical ventilation at TVs of 3,6 and $9 \mathrm{~mL} / \mathrm{kg}$ at baseline and after lung lavage (decreased lung compliance). Dynamic preload indicators were derived afterwards. Results. With increasing TV the change in Paw and CVP increased linearly with $2.5 \pm 0.6$ and $0.16 \pm 0.06$ $\mathrm{mmHg} / \mathrm{ml} / \mathrm{kg}$, respectively. As a result of the lavages, the total respiratory compliance decreased from $4.3 \pm 1.9$ to $2.5 \pm 0.6 \mathrm{ml} / \mathrm{cmH} 2 \mathrm{O}$. The percentage of airway pressure that was transmitted to the vena cava did not change over the different tidal volumes $(\mathrm{p}=0.41)$. Lavage of the lungs however, did decrease
} 
this percentage $\left(\mathrm{p}_{i} 0.05\right)$. Furthermore, stroke volume variation was more pronounced at higher levels of TV and decreased lung compliance did not influence the stroke volume variation. The change in the pressure measured at the peripheral vein was $8320.39 \%$ of the change in CVP and the change in venous returning pressure gradient was related to the value of the patients stroke volume variation.

Conclusion. Tidal volume, and not airway pressure or lung compliance is the key determinant of changes in intrathoracic pressure and dynamic preload indicators. Furthermore, in non-preload dependent patients, venous returning driving pressure is not influenced by changes in intrathoracic pressure. 


\subsection{Introduction}

Intermittent positive airway pressure during mechanical ventilation periodically increases intra-thoracic pressure. This temporary increase, decreases output of the right heart and, after an initial increase, also decrease output of the left heart [1]. These ventilation-induced changes, quantified by the dynamic preload indicators pulse pressure variation (PPV), systolic pressure variation (SPV) and stroke volume variation (SVV), are related to the position of the patients heart on the Frank-Starling curve and have been shown to predict volume responsiveness in mechanically ventilated adult patients $[2,3]$. Also in children, these arterial pressure variations have been found. However, in children, the evidence about their predictive value is poor and suggest that dynamic parameters extracted from the arterial pressure waveform on the whole do not predict fluid responsiveness [4]. Besides the low heart rate to respiratory rate ratio, possible explanations for this are the higher chest wall and lung compliance in children [5], and a more compliant arterial tree [6, 7].

Also in adults, abnormal lung compliance (e.g. as a result of acute respiratory distress syndrome (ARDS)) is considered to limit the predictive value of dynamic preload indicators $[8,9]$. Especially in patients with ARDS, adequate fluid management, and thus adequate predictors for fluid responsiveness, are essential. On the one hand because sufficient intravascular fluid is required in order to compensate for the hemodynamic effects of positive end-expiratory pressure (PEEP) and to maintain adequate hemodynamic conditions in the case of associated septic shock. On the other hand, fluid overloading may result in additional extravascular lung water and hence in worsening of hypoxemia and further alteration of lung mechanics [10,11], also in children [12].

Because of the complex cardiopulmonary interactions in ARDS-conditions, the current literature is not unanimous about the contribution of decreased lung compliance and opposite conclusions has been drawn about the usefulness of dynamic indices in ARDS. Some claim that lung compliance is a key determinant of the dynamic indices and that the dynamic indices might still be useful in ARDS despite the use of low tidal volume (TV) [16]. They argue that a decrease in lung compliance will increase the airway driving pressure, resulting in sufficient swings in transpulmonary pressure to maintain the predictive value of the dynamic indices [9, 13-15]. Others claim that lung compliance does not matter and that dynamic indices must not be used in case of ARDS because the low tidal volume is accompanied with small variations in pleural pressure which might not be sufficient to trigger significant preload variations, even in cases of preload responsiveness [8, 17-19].

To improve our knowledge about the underlying physiology of heart-lung interaction in children, and more specifically during ARDS, the primary aim of the present animal study was to show to what extent the airway pressure is distributed to the central venous pressure (CVP), how this influences mechanically ventilated induced 
preload indicators, and how this distribution is influenced by changes in tidal volume and lung compliance. The secondary objective was to show the influence of positive airway pressure on the pressure gradient between peripheral and central veins, representing the driving pressure for the venous return.

\subsection{Materials and Methods}

\subsubsection{General}

In accordance with Dutch national legislation concerning guidelines for the care and use of laboratory animals, and with approval of the local ethics committee on animal research of the Radboud university medical center (Radboudumc, Licence number RU-DEC 2010-034; CDL-projectnumber 33078), eleven newborn lambs were studied under general anesthesia. Premedication consisted of the intramuscular administration of midazolam $(0.2 \mathrm{mg} / \mathrm{kg})$, ketamine $(10 \mathrm{mg} / \mathrm{kg})$ and i.v. administration of propofol $(2 \mathrm{mg} / \mathrm{kg})$. General anesthesia was maintained using inhalation of isoflurane (1-1.5 vol\%) and continuous i.v. administration of sufentanyl $(20 \mu \mathrm{g} / \mathrm{kg} / \mathrm{h})$, midazolam $(0.2 \mathrm{mg} / \mathrm{kg} / \mathrm{h})$, ketamine $(10 \mathrm{mg} / \mathrm{kg} / \mathrm{h})$, and pancuronium $(0.02 \mathrm{mg} / \mathrm{kg} / \mathrm{h})$ after a loading dose $(0.05 \mathrm{mg} / \mathrm{kg})$. The depth of anaesthesia was repeatedly assessed by painful stimuli and clinical parameters such as heart rate, spontaneous ventilation, and elevated arterial pressure. The depth of anaesthesia was adjusted when necessary using isoflurane. During the experiment continuous i.v. dextrose $(10 \% 2 \mathrm{ml} / \mathrm{kg} / \mathrm{h})$ was administered. The lambs were orotracheally intubated using a 4-6 mm (inner diameter) cuffed endotracheal tube (Kruse, Marslev, Denmark). The lungs were mechanically ventilated in a pressure-controlled mode using tidal volumes of $10 \mathrm{ml} / \mathrm{kg}$ (Datex-Ohmeda anesthesia machine) and an inspiratory-to-expiratory ratio of 1:2. Normocapnia, guided by capnography with the NICO Respiratory Monitor (Model 8100, Respironics, Pittsburgh, USA), was achieved by adjusting the minute volume ventilation to maintain an end-tidal $\mathrm{CO} 2$ tension between 4.0 and $5.5 \mathrm{kPa}$. Impaired oxygenation was treated by adjusting the positive end expiratory pressure (PEEP) and fraction of inspired oxygen ( $\mathrm{FiO} 2)$ to maintain the oxygen saturation $>95 \%$. A servo-controlled heating mattress and an external heating lamp were used to provide core temperature stability (38-40C). At the end of the experiment, the animals were killed with an overdose of pentobarbital ( $150 \mathrm{mg} / \mathrm{kg}$, i.v.).

\subsubsection{Instrumentation}

Immediately after induction of anesthesia the animals received a femoral artery catheter (20 Ga, single lumen, $12 \mathrm{~cm}$, Arrow, Germany), a femoral central venous catheter (5Fr, 2 lumen, $13 \mathrm{~cm}$, Arrow, Germany) and a catheter in a peripheral vein, for pressure measurements. A left-sided thoracotomy was performed and the remains of the 
native ductus arteriosus was ligated. An ultrasound transit time perivascular flow probe (PAX series, Transonic Systems, Ithaca, NY) was placed around the main pulmonary artery (probe 10 or $12 \mathrm{~mm}$ ) to measure continues cardiac output (CO) and stroke volume (SV). The flow probes were checked for zero flow value directly postmortem. Ultrasound transit time flow probes use a two-way ultrasound technique. By calculating the difference between transit times upstream and downstream, the blood flow (Q) is measured. Care was taken to avoid air within the flow probe by applying sufficient quantities of acoustic gel. After the placement of the flow probe and the intrathoracic catheters, the thorax was closed. The animals were positioned either supine or lying on the right side throughout the experiment.

\subsubsection{Measurements}

During the experiment, arterial pressure (ABP), central venous pressure (CVP), peripheral vein pressure (PVP), electrocardiogram, stroke volume (SV), heart rate, respiratory frequency, tidal volume, airway pressures, and body core temperature were recorded simultaneously with a $200-\mathrm{Hz}$ sampling rate using a computer system with special biomedical registration software (Poly, Inspektor Research Systems, Amsterdam, The Netherlands). From the recorded ABP and SV, the PPV, SPV and SVV were calculated offline, defined as the mean value of the relative difference in pulse pressure, systolic pressure and stroke volume over one breath, averaged over a period of 5 breaths.

\subsubsection{Protocol}

After the instrumentation and 30 minutes of stabilization, the animals were ventilated with 3 different values of tidal volume $(3,6$ and $9 \mathrm{ml} / \mathrm{kg}$ ) and subsequently, three 20-second-periods of inspiratory holds were performed with increasing plateau pressures. After this, the lambs underwent repetitive saline lavages $(10-30 \mathrm{ml} / \mathrm{kg}$ $37^{\circ} \mathrm{C} \mathrm{NaCl} 0.9 \%$ ) of the lung in order to induce surfactant depletion and provoke acute lung injury (ALI). Before the lavages, the lambs were pre-oxygenated with $\mathrm{FiO} 2$ 1.0. Between the lavages the PEEP and minute volume ventilation were increased to maintain oxygen saturation and end tidal $\mathrm{CO} 2$ within the normal range. The saline lavages were performed in either one or two sessions of $10-30 \mathrm{ml} / \mathrm{kg}$ right after each other, depending on the recovery of the lambs during the procedure. After each lavage procedure and 30 minutes of stabilization, the measurements with 3 different tidal volumes and inspiratory holds were repeated. A total of 3 up to 6 lavage sessions were performed. A blood transfusion was administered if the hemoglobin $\mathrm{Hb}$ ) content of the blood was $<3.5 \mathrm{mmol} / \mathrm{l}$, to optimize the oxygen delivery to the tissues. Dobutamine or epinephrine was administered when indicated. 

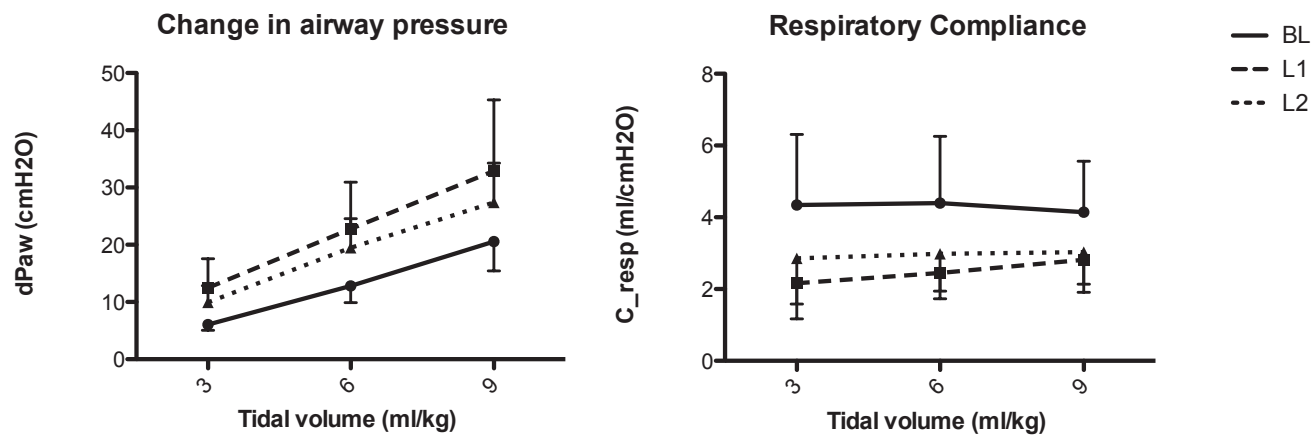

Figure 1: part a: the change in airway pressure $(\mathrm{Paw})$ due to positive pressure ventilation. Part b: the total respiratory compliance (C-resp). Both during the different levels of tidal volume (horizontal axis) and at the different stages of lung lavages $(B L=$ baseline, $L 1=$ after lung lavage 1, L2 = after lung lavage 2).

\subsubsection{Statistical analysis}

Differences in the effect of changes in TV and chest wall compliance were compared with repeated measures two-way analysis of variance (ANOVA) since data was normally distributed, including Bonferroni tests for subgroups. Paired Students t-tests were used to compare the means of two groups. P-values $<0.05$ were considered statistically significant. Data is presented as meanstandard deviation and statistical analysis was performed using SPSS 19 for Mac (SPSS Inc., Chicago, Ill, USA) and Prism 5 for Mac (Graphpad Software Inc, La Jolla, CA, USA).

\subsection{Results}

Eleven newborn lambs $(8.65 \pm 2.29 \mathrm{~kg}$ body weight and age $17.0 \pm 4.42$ days) were studied. At baseline, mechanical ventilation at $3 \mathrm{ml} / \mathrm{kg}$ resulted in a change in airway pressure of $6.1 \pm 1.0 \mathrm{cmH} 2 \mathrm{O}$. With increasing tidal volumes, the change in Paw increased $(\mathrm{p}<0.01)$ linearly with $2.5 \pm 0.6 \mathrm{mmHg} / \mathrm{ml} / \mathrm{kg}$ to $20.6 \pm 5.1 \mathrm{mmHg}$ at $9 \mathrm{ml} / \mathrm{kg}$, see Figure 1a.

As a result of the repetitive saline lavages to induce ALI, the total respiratory compliance decreased from $4.3 \pm 1.9 \mathrm{ml} / \mathrm{cmH} 2 \mathrm{O}$ at baseline to $2.5 \pm 0.6$ at stage I (L1, $\mathrm{p}<0.01)$. Respiratory compliance did not decrease any further after the second lavage (L2, respiratory compliance at $\mathrm{L} 2$ was $2.8 \pm 1.1, \mathrm{p}=0.15$ ), see Figure $1 \mathrm{~b}$. At stage I and II (L1 and L2), the change in airway pressure was significantly higher than at baseline $(10.0 \pm 2.9 \mathrm{cmH} 2 \mathrm{O}$ and linearly increasing to $27.4 \pm 6.9 \mathrm{mmHg}$ at $9 \mathrm{ml} / \mathrm{kg}$ for 
Change in central venous pressure

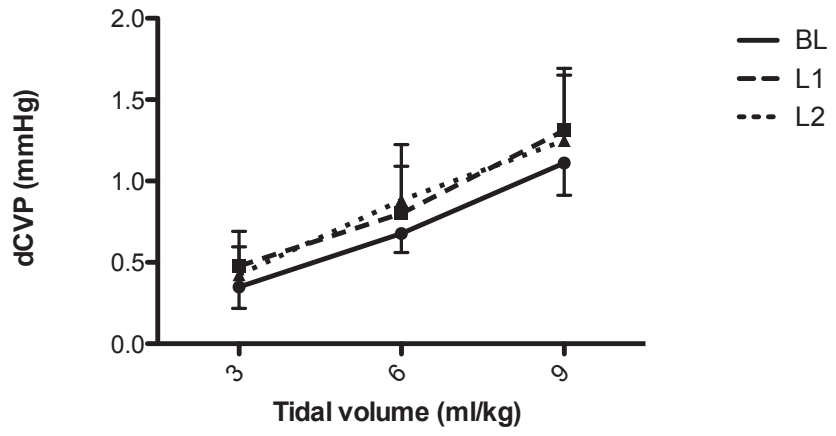

Figure 2: the change in central venous pressure (dCVP) due to positive pressure ventilation during the different levels of tidal volume $(3,6$ and $9 \mathrm{ml} / \mathrm{kg})$ and at the different stages of lung lavages (BL= baseline, $L 1=$ after lung lavage $1, L 2=$ after lung lavage 2$)$.

\section{L1, see Figure 1a).}

The change in CVP also increased significantly $(\mathrm{p}<0.01)$ and linearly as a result of the increasing tidal volumes with $0.16 \pm 0.06 \mathrm{mmHg} / \mathrm{ml} / \mathrm{kg}$, respectively, see Figure 2. There was no change as a result of the decreased respiratory compliance $(\mathrm{p}=0.1)$. The percentage of airway pressure that was transmitted to the vena cava at baseline was $8.7 \pm 2.4 \%$, see Figure 3a. This percentage did not change over the different tidal volumes $(\mathrm{p}=0.41)$. Lavage of the lungs significantly decreased this percentage to $6.9 \pm 2.3$ at $\mathrm{L} 1(\mathrm{p}<0.05)$. There was no significant difference between $\mathrm{L} 1$ and L2 ( $\mathrm{p}=0.28)$. With increasing tidal volume, the change in CVP divided by tidal volume remains constant $(\mathrm{p}=0.17$ ). Also no difference was found for the change in CVP divided by tidal volume over the different stages of respiratory compliance $(\mathrm{p}=0.37)$ (see Figure $3 \mathrm{~b})$.

The increased changes in CVP due to the mechanical ventilation with increasing tidal volume resulted in a statistically significant decrease in stroke volume index ( $p=0.03$, see Figure 4). There was no change in SVI as a result of the lung lavages $(\mathrm{p}=0.07)$. At baseline, a prolonged increase in intrathoracic pressure during an inspiratory hold manoeuvre at the three different levels of tidal volumes resulted in a decrease of SVI of $0.5 \pm 0.5,0.7 \pm 0.7$ and $1.5 \pm 0.8 \mathrm{ml} / \mathrm{m} 2$ for 3,6 , and $9 \mathrm{ml} / \mathrm{kg}$, respectively (all $\mathrm{p}<0.05$, data no shown).

The cyclic changes in intrathoracic pressure during MV resulted in arterial pressure variations (PPV, SPV and SVV) that were more pronounced at higher levels of TV. For PPV and SVV, decreased lung compliance had no influence ( $\mathrm{p}=0.13$ and 0.52 , respectively), see Figure 5. Figure 6 shows the SVV divided by TV (part a) and 
Transmission of airway pressure to CVP

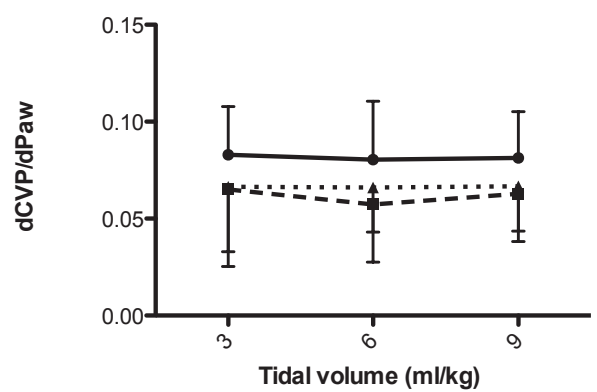

Change in CVP divided by tidal volume
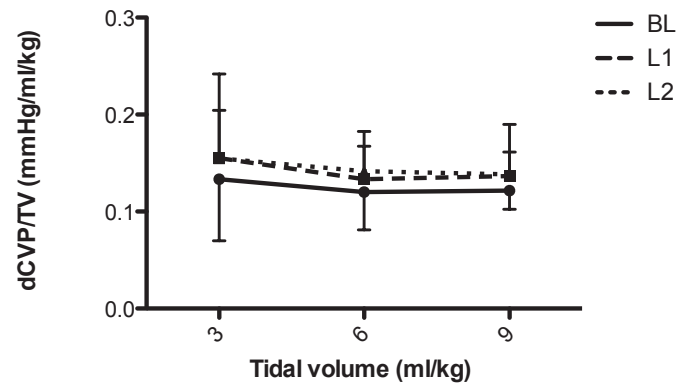

Figure 3: part a: the part of the airway pressure (Paw) that was transmitted to the central venous pressure $(C V P)$. Part b: the change in CVP divided by the tidal volume. Both during the different levels of tidal volume and at the different stages of lung lavages ( $B L=$ baseline, L1 = after lung lavage 1, L2 = after lung lavage 2).

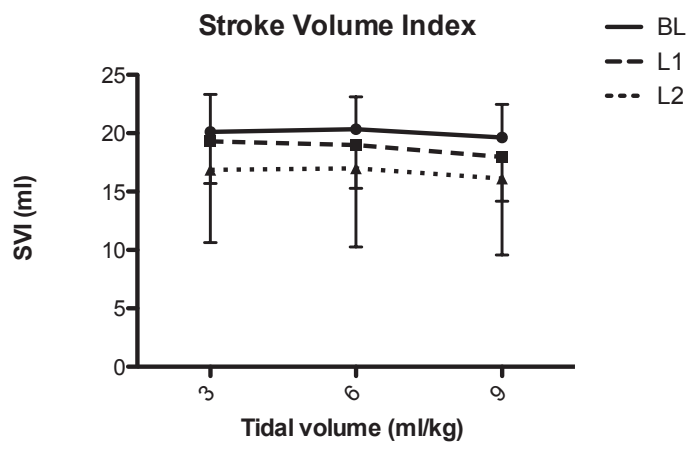

Figure 4: Stroke volume index (SVI) during the different levels of tidal volume and at the different stages of lung lavages $(B L=$ baseline, $L 1=$ after lung lavage 1, L2 = after lung lavage 2). 
PPV

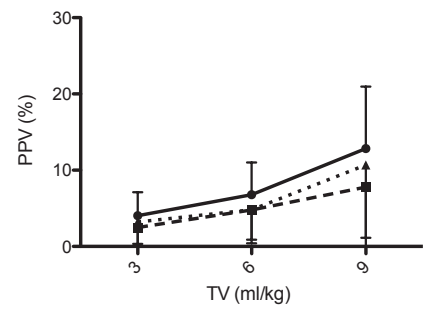

SPV

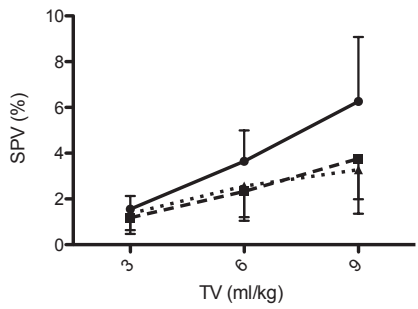

SW

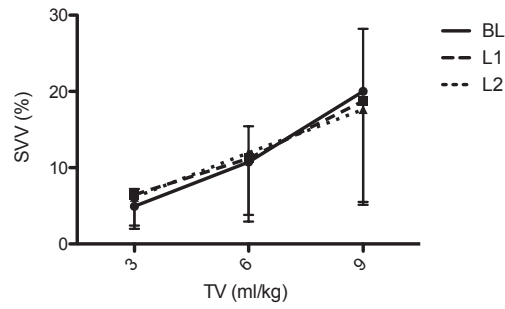

Figure 5: Dynamic indices (pulse pressure variation, ppv; systolic pressure variation, SPV and stroke volume variation, SVV) during different levels of tidal volume (TV) and pleural compliance (at baseline [BL] and lung lavage [L1 and L2]).

SW/TV

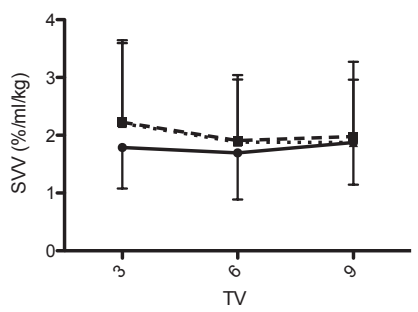

SVV/dCVP

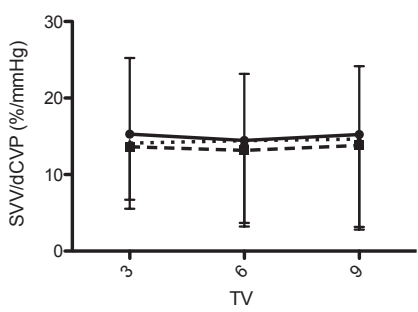

SW/Paw

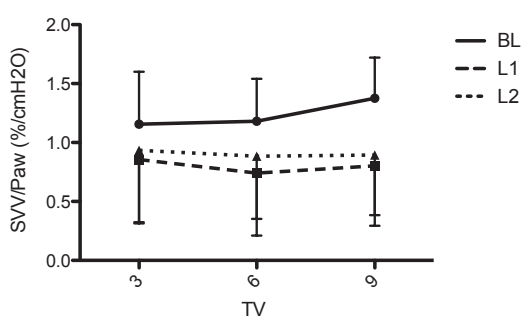

Figure 6: Stroke volume variation (SVV) divided by tidal volume (TV, part a), central venous pressure (CVP, part $b)$ and airway pressure (Paw, part c) during the different levels of tidal volume and at the different stages of lung lavages $(B L=$ baseline, $L 1=$ after lung lavage 1 , L2 = after lung lavage 2). 

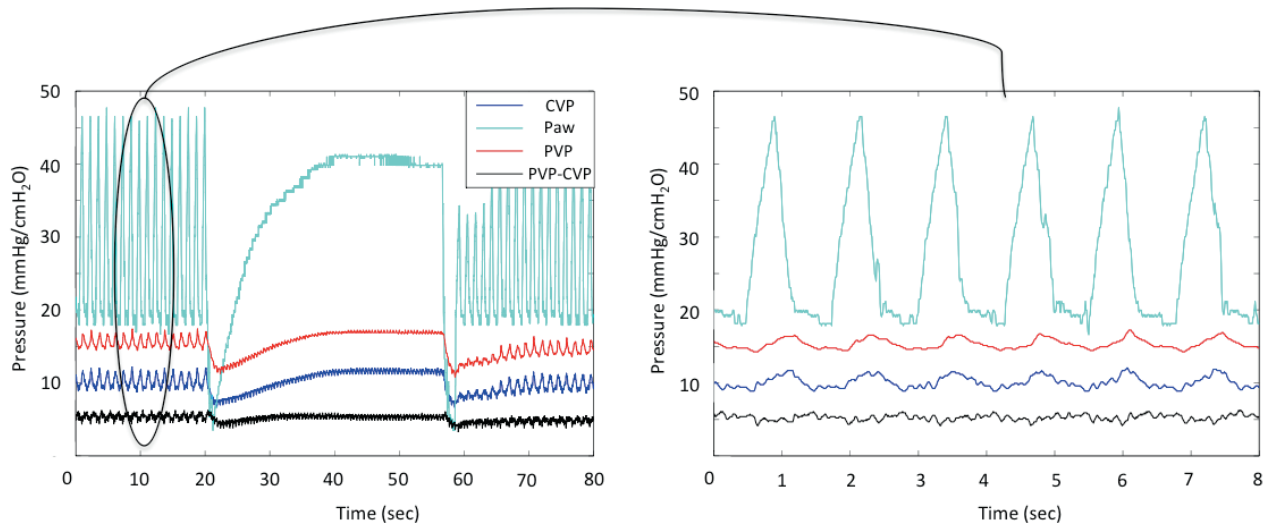

Figure 7: Representative sample of the airway pressure (Paw, lime, in cmH2O), central venous pressure (CVP, blue, in $\mathrm{mmHg}$ ) and peripheral venous pressure ( $\mathrm{PVP}$, red, in $\mathrm{mmHg})$ before, during and after an inspiratory hold (part a). The black line is the difference between the PVP and the CVP and represents the venous returning driving pressure. Part $b$ is $a$ magnification of the part before the inspiratory hold (circled area).

relation between the percentage of distributed dCVP and SW

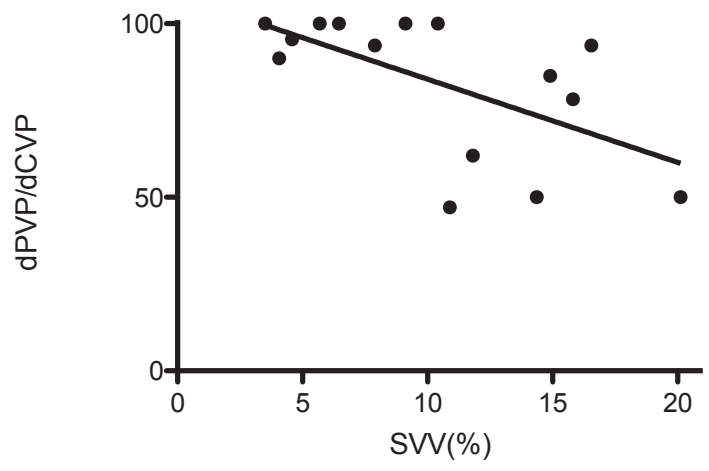

Figure 8: Relation between the percentage of distributed change in central venous pressure $(C V P)$ to the peripheral venous pressure $(P V P)$ and the stroke volume variation (SVV). 
CVP (part b) over the different tidal volumes and respiratory compliances. Both TV and respiratory compliance had no significant influence. SVV divided by Paw was significantly different at the different stages of respiratory compliance $(\mathrm{p}<0.01$, see Figure 6, part c).

The change in the pressure measured at the peripheral vein (PVP) was $83 \pm 20.39 \%$ of the change in CVP and the PVP follows the CVP closely. Figure 7 shows a representative sample of the CVP (in blue) and PVP (in red) before, during and after an inspiratory hold (airway pressure is show in lime). The black line, indicating the difference between the PVP and CVP and representing the driving pressure of the venous return, only fluctuates minimally at the start of a pressure increase/decrease which indicates only a small time-delay for this distribution of CVP to the PVP $(<0.1$ sec). Figure 8 shows the relation between the percentage of distributed change in CVP to the PVP and the SVV, as a measure of fluid responsiveness.

\subsection{Discussion}

The present animal study demonstrates that increasing tidal volumes will linearly increase airway pressure, central venous pressure and stroke volume variation. Decreased lung compliance due to lung lavage, did increase the airway driving pressure, but did not influence the change in central venous pressure, nor did it influenced stroke volume variation. The transmission of airway pressure to the central venous pressure on the other hand, did decreased as a result of the decreased lung compliance, resulting in an increased transpulmonary pressure. These findings demonstrate that tidal volume, and not airway pressure or lung compliance, is the key determinant of the change in intrathoracic pressure and stroke volume variation. Our data also shows that the venous returning driving pressure is not influenced by a change in intrathoracic pressure in patients with a low value of stroke volume variation because the peripheral pressure follows the central venous pressure closely.

In our study, the finding that central venous pressure did not change with increasing airway pressure (remaining the same amount of tidal volume) while the transmission of airway pressure to the central venous pressure did change with decreasing lung compliance, confirms the findings of recent and older literature indicating that tidal volume is more relevant than airway pressure when it comes to intrathoracic pressure changes [20-22]. This is because although airway pressure is of course related to pleural pressure, high lung stiffness prevents alveolar pressure from being transmitted to the pleural space and basic physiology (Ohms law) tells us that it is the tidal volume divided by chest wall compliance that determines the change in pleural pressure. Our study also confirms the the previously obtained results that the higher the tidal volume, the higher the variation of stroke volume or pulse pressure [23-27]. However, these dynamic preload indicators that can be used 
for fluid responsiveness are not only caused by a change in pleural pressure, they are also influenced by the transpulmonary pressure. While an increased pleural pressure causes the right ventricular preload and the left ventricular afterload to decrease, an increased transpulmonary pressure increases right ventricular afterload and left ventricular preload [1, 28-30]. For this reason, it was hypothesized that although the pleural pressure swings are lower since in ARDS patients low tidal volumes are used, dynamic preload indicators might still be sufficient and useful because of the increased transpulmonary pressure swings (due to the decreased lung compliance) $[9,13,14]$.

Our results however, clearly show that with increasing transpulmonary pressure (due to the decreased lung compliance and increased airway pressure) without an increase in central venous pressure, the stroke volume variation did not changed. This is also confirmed by the finding that there is no difference in stroke volume variation divided by tidal volume or stroke volume divided by the change in central venous pressure over the different stages of lung compliance. On the contrary, the stroke volume variation divided by airway pressure did change after lavage of the lungs, indicating that it is the tidal volume, and not the airway pressure that determines the value of the dynamic preload indicators. This confirms the argumentations and findings of others, claiming that the inability of dynamic indices to predict fluid responsiveness in ARDS is related to the use of low tidal volumes and that this is not compensated by an increase in transpulmonary pressure due to the increased lung compliance $[8,17,18,31,32]$. For this reason, the high chest wall compliance in children is more likely to be a limitation regarding the usability of dynamic indices in order to predict fluid responsiveness rather than the high lung compliance. Our findings also supports the suggestion to index dynamic preload predictors to the used tidal volume [33], and not to the airway driving pressure [26].

One of the components of the decreased cardiac output during positive pressure ventilation, is considered to be the decrease in right ventricular preload caused by a decrease in the driving pressure for venous return, which is de gradient from the mean systemic filling pressure to the central venous pressure [1,28-30]. For this reason, the mean systemic filling pressure has repeatedly been suggested as an important parameter during fluid management [34-37]. In the recent years, some methods are presented to measure the mean systemic filling pressure without inducing a circulatory stop [38]. This allows us to use the mean systemic filling pressure during fluid management at the bedside. In order to describe the influence of intermittent and prolonged increased intrathoracic pressure on the pressure gradient, we measured the difference between the central venous pressure and the pressure in a peripheral vein during positive pressure ventilation and during an inspiratory hold. Our results clearly show that the peripheral pressure, after a small time delay, closely follows the changes in central venous pressure, indicating that the pressure gradient for venous 
return did not change during positive pressure ventilation, nor during prolonged period of increased intrathoracic pressure. This is in accordance to the results of others studies [39-43] and indicates that the decrease in cardiac output is caused by an increase in resistance of the venous conduits. In addition, we also found that in patients with a high stroke volume variation, the pressure gradient did decrease, indicating that in fluid responsive or hypovolemic patients, the decrease in cardiac output is caused by a decrease in the pressure gradient for venous return.

\subsubsection{Limitation}

Several limitation of our study needs to be addressed. First of all, although we assume that the compliance of the lungs was decreased as a result of the lung lavages, we were not actually able to measure the compliance of the lung. On the other hand, as the airway driving pressure clearly increased after lavage of the lung, and there is no reason to think why this lung lavage would also have influenced the chest wall compliance, the assumption that the compliance of the lungs did decreased is very likely to be right.

Second, we did not measure fluid responsiveness, so we are only describe the influence of lung compliance on the intrathoracic pressure and the value of the dynamic indices and not to the change in accuracy regarding the prediction of fluid responsiveness. The study was not designed for this since our primary goal was to improve our knowledge about the underlying physiology of heart-lung interaction during ARDS. Finally, because we used newborn animals for this study, caution is needed when extrapolating these results to adults. On the other hand, the presented results primarily involve basic physiology, and these will also holds for adults since they involve the basic laws of physics.

\subsection{Conclusion}

Tidal volume, and not airway pressure or lung compliance, is the key determinant of the change in intrathoracic pressure and dynamic preload indicators. Furthermore, in non-preload dependent patients, venous returning driving pressure is not influenced by changes in intrathoracic pressure whereas in preload dependent patients it is.

\section{References}

[1] F. Michard, "Changes in arterial pressure during mechanical ventilation," Anesthesiology, vol. 103, no. 2, pp. 419-28, 2005.

[2] S. Magder, "Clinical usefulness of respiratory variations in arterial pressure," Am J Respir Crit Care Med, vol. 169, no. 2, pp. 151-5, 2004. 
[3] P. E. Marik, R. Cavallazzi, T. Vasu, and A. Hirani, "Dynamic changes in arterial waveform derived variables and fluid responsiveness in mechanically ventilated patients: a systematic review of the literature," Crit Care Med, vol. 37, no. 9, pp. 2642-7, 2009.

[4] H. Gan, M. Cannesson, J. R. Chandler, and J. M. Ansermino, "Predicting fluid responsiveness in children: a systematic review," Anesthesia and analgesia, vol. 117, no. 6, pp. 1380-92, 2013.

[5] C. Papastamelos, H. B. Panitch, S. E. England, and J. L. Allen, "Developmental changes in chest wall compliance in infancy and early childhood," Journal of applied physiology, vol. 78, no. 1, pp. 179-84, 1995.

[6] H. Senzaki, M. Akagi, T. Hishi, A. Ishizawa, M. Yanagisawa, S. Masutani, T. Kobayashi, and S. Awa, "Age-associated changes in arterial elastic properties in children," European journal of pediatrics, vol. 161, no. 10, pp. 547-51, 2002.

[7] M. R. Roach and A. C. Burton, "The effect of age on the elasticity of human iliac arteries," Canadian journal of biochemistry and physiology, vol. 37, no. 4, pp. 557-70, 1959.

[8] K. Lakhal, S. Ehrmann, D. Benzekri-Lefevre, I. Runge, A. Legras, P. F. Dequin, E. Mercier, M. Wolff, B. Regnier, and T. Boulain, "Respiratory pulse pressure variation fails to predict fluid responsiveness in acute respiratory distress syndrome," Critical care, vol. 15, no. 2, p. R85, 2011.

[9] X. Monnet, A. Bleibtreu, A. Ferre, M. Dres, R. Gharbi, C. Richard, and J. L. Teboul, "Passive leg-raising and end-expiratory occlusion tests perform better than pulse pressure variation in patients with low respiratory system compliance," Critical care medicine, vol. 40, no. 1, pp. 152-7, 2012.

[10] J. J. Geraets, I. J. de Groot, M. E. Goossens, C. P. de Bruijn, R. A. de Bie, W. J. van den Heuvel, and G. J. Dinant, "Comparison of two recruitment strategies for patients with chronic shoulder complaints," The British journal of general practice : the journal of the Royal College of General Practitioners, vol. 56, no. 523, pp. 127-33, 2006.

[11] Y. Sakr, J. L. Vincent, K. Reinhart, J. Groeneveld, A. Michalopoulos, C. L. Sprung, A. Artigas, and V. M. Ranieri, "High tidal volume and positive fluid balance are associated with worse outcome in acute lung injury," Chest, vol. 128, no. 5, pp. 3098-108, 2005.

[12] A. K. Ewer, W. Tyler, A. Francis, D. Drinkall, and J. O. Gardosi, "Excessive volume expansion and neonatal death in preterm infants born at 27-28 weeks 
gestation," Paediatric and perinatal epidemiology, vol. 17, no. 2, pp. 180-6, 2003.

[13] J. L. Teboul and A. Vieillard-Baron, "Clinical value of pulse pressure variations in ards. still an unresolved issue?" Intensive care medicine, vol. 31, no. 4, pp. 499-500, 2005.

[14] J. Mallat, M. Lemyze, and D. Thevenin, "Ability of respiratory pulse pressure variation to predict fluid responsiveness in ards: still an unanswered question?" Critical care, vol. 15, no. 3, p. 432; author reply 432, 2011.

[15] C. C. Huang, J. Y. Fu, H. C. Hu, K. C. Kao, N. H. Chen, M. J. Hsieh, and Y. H. Tsai, "Prediction of fluid responsiveness in acute respiratory distress syndrome patients ventilated with low tidal volume and high positive end-expiratory pressure," Crit Care Med, vol. 36, no. 10, pp. 2810-6, 2008.

[16] ARDS-network, "Ventilation with lower tidal volumes as compared with traditional tidal volumes for acute lung injury and the acute respiratory distress syndrome. the acute respiratory distress syndrome network," $N$ Engl J Med, vol. 342, no. 18, pp. 1301-8, 2000.

[17] J. Y. Lefrant and D. De Backer, "Can we use pulse pressure variations to predict fluid responsiveness in patients with ards?" Intensive Care Med, vol. 35, no. 6, pp. 966-8, 2009.

[18] K. Lakhal, S. Ehrmann, and T. Boulain, "Pulse pressure variation: does lung compliance really matter?" Critical care medicine, vol. 40, no. 5, pp. 1691; author reply 1691-2, 2012.

[19] D. De Backer, S. Heenen, M. Piagnerelli, M. Koch, and J. L. Vincent, "Pulse pressure variations to predict fluid responsiveness: influence of tidal volume," Intensive Care Med, vol. 31, no. 4, pp. 517-23, 2005.

[20] F. Jardin, B. Genevray, D. Brun-Ney, and J. P. Bourdarias, "Influence of lung and chest wall compliances on transmission of airway pressure to the pleural space in critically ill patients," Chest, vol. 88, no. 5, pp. 653-8, 1985.

[21] J. A. Romand, W. Shi, and M. R. Pinsky, "Cardiopulmonary effects of positive pressure ventilation during acute lung injury," Chest, vol. 108, no. 4, pp. 1041 8,1995 .

[22] J. C. Chapin, J. B. Downs, M. E. Douglas, E. J. Murphy, and B. C. Ruiz, "Lung expansion, airway pressure transmission, and positive end-expiratory pressure," Archives of surgery, vol. 114, no. 10, pp. 1193-7, 1979. 
[23] H. K. Kim and M. R. Pinsky, "Effect of tidal volume, sampling duration, and cardiac contractility on pulse pressure and stroke volume variation during positive-pressure ventilation," Crit Care Med, vol. 36, no. 10, pp. 2858-62, 2008.

[24] D. A. Reuter, J. Bayerlein, M. S. Goepfert, F. C. Weis, E. Kilger, P. Lamm, and A. E. Goetz, "Influence of tidal volume on left ventricular stroke volume variation measured by pulse contour analysis in mechanically ventilated patients," Intensive Care Med, vol. 29, no. 3, pp. 476-80, 2003.

[25] C. Charron, C. Fessenmeyer, C. Cosson, J. X. Mazoit, J. L. Hebert, D. Benhamou, and A. R. Edouard, "The influence of tidal volume on the dynamic variables of fluid responsiveness in critically ill patients," Anesth Analg, vol. 102, no. 5, pp. 1511-7, 2006.

[26] F. Vallee, J. C. Richard, A. Mari, T. Gallas, E. Arsac, P. S. Verlaan, B. Chousterman, K. Samii, M. Genestal, and O. Fourcade, "Pulse pressure variations adjusted by alveolar driving pressure to assess fluid responsiveness," Intensive Care Med, vol. 35, no. 6, pp. 1004-10, 2009.

[27] J. Mesquida, H. K. Kim, and M. R. Pinsky, "Effect of tidal volume, intrathoracic pressure, and cardiac contractility on variations in pulse pressure, stroke volume, and intrathoracic blood volume," Intensive care medicine, vol. 37, no. 10, pp. 1672-9, 2011.

[28] F. Michard and J. L. Teboul, "Using heart-lung interactions to assess fluid responsiveness during mechanical ventilation," Crit Care, vol. 4, no. 5, pp. 282-9, 2000 .

[29] F. Feihl and A. F. Broccard, "Interactions between respiration and systemic hemodynamics. part i: basic concepts," Intensive Care Med, vol. 35, no. 1, pp. 45-54, 2009.

[30] M. R. Pinsky, "The hemodynamic consequences of mechanical ventilation: an evolving story," Intensive care medicine, vol. 23, no. 5, pp. 493-503, 1997.

[31] L. Muller, G. Louart, P. J. Bousquet, D. Candela, L. Zoric, J. E. de La Coussaye, S. Jaber, and J. Y. Lefrant, "The influence of the airway driving pressure on pulsed pressure variation as a predictor of fluid responsiveness," Intensive care medicine, vol. 36, no. 3, pp. 496-503, 2010.

[32] J. Renner, E. Cavus, P. Meybohm, P. Tonner, M. Steinfath, J. Scholz, G. Lutter, and B. Bein, "Stroke volume variation during hemorrhage and after fluid loading: impact of different tidal volumes," Acta anaesthesiologica Scandinavica, vol. 51, no. 5, pp. 538-44, 2007. 
[33] S. T. Vistisen, J. Koefoed-Nielsen, and A. Larsson, "Should dynamic parameters for prediction of fluid responsiveness be indexed to the tidal volume?" Acta Anaesthesiol Scand, 2009.

[34] A. C. Guyton, A. W. Lindsey, and B. N. Kaufmann, "Effect of mean circulatory filling pressure and other peripheral circulatory factors on cardiac output," The American journal of physiology, vol. 180, no. 3, pp. 463-8, 1955.

[35] W. G. Parkin, "Volume state control - a new approach," Critical care and resuscitation : journal of the Australasian Academy of Critical Care Medicine, vol. 1, no. 3, pp. 311-21, 1999.

[36] J. R. Jansen, J. J. Maas, and M. R. Pinsky, "Bedside assessment of mean systemic filling pressure," Curr Opin Crit Care, 2010.

[37] M. Cecconi, H. D. Aya, M. Geisen, C. Ebm, N. Fletcher, R. M. Grounds, and A. Rhodes, "Changes in the mean systemic filling pressure during a fluid challenge in postsurgical intensive care patients," Intensive care medicine, vol. 39, no. 7, pp. 1299-305, 2013.

[38] J. J. Maas, M. R. Pinsky, B. F. Geerts, R. B. de Wilde, and J. R. Jansen, "Estimation of mean systemic filling pressure in postoperative cardiac surgery patients with three methods," Intensive care medicine, vol. 38, no. 9, pp. 1452-60, 2012.

[39] T. Luecke and P. Pelosi, "Clinical review: Positive end-expiratory pressure and cardiac output," Crit Care, vol. 9, no. 6, pp. 607-21, 2005.

[40] H. E. Fessler, R. G. Brower, R. A. Wise, and S. Permutt, "Effects of positive end-expiratory pressure on the gradient for venous return," The American review of respiratory disease, vol. 143, no. 1, pp. 19-24, 1991.

[41] S. Nanas and S. Magder, "Adaptations of the peripheral circulation to peep," The American review of respiratory disease, vol. 146, no. 3, pp. 688-93, 1992.

[42] H. Jellinek, H. Krenn, W. Oczenski, F. Veit, S. Schwarz, and R. D. Fitzgerald, "Influence of positive airway pressure on the pressure gradient for venous return in humans," Journal of applied physiology, vol. 88, no. 3, pp. 926-32, 2000.

[43] P. C. van den Berg, J. R. Jansen, and M. R. Pinsky, "Effect of positive pressure on venous return in volume-loaded cardiac surgical patients," Journal of applied physiology, vol. 92, no. 3, pp. 1223-31, 2002. 


\title{
7 \\ Dynamic preload indicators decrease when the abdomen is opened
}

\author{
M. van Lavieren, J. Veelenturf, C. Hofhuizen, M. van der Kolk, J.G. van der \\ Hoeven, P.Pickker, J. Lemson and B. Lansdorp. \\ BMC Anesthesiology: submitted
}

Background. Optimizing cardiac stroke volume during major surgery decreases the incidence of postoperative complications. Because dynamic preload indicators are strongly correlated with stroke volume, it is suggested that these indices can be used for goal directed fluid therapy. However, threshold values of these indicators depend on many factors that are influenced by surgery, including opening of the abdomen. The aim of this study was therefore to assess the effect of opening the abdomen on arterial pressure variations in patients undergoing abdominal surgery.

Methods. Blood pressure and bladder pressure were continuously recorded just before and after opening of the abdomen in patients undergoing elective laparotomy. Based on waveform analysis of the blood pressure, the stroke volume index, pulse pressure variation (PPV) and stroke volume variation (SVV) were calculated.

Results. Thirteen patients were included. After opening the abdomen, PPV and SVV decreased from $11.5 \pm 5.8 \%$ to $6.4 \pm 2.9 \%$ ( $<<0.005$, a relative decrease of $40 \pm 19 \%$ ) and $12.7 \pm 6.1 \%$ to $4.8 \pm 1.6 \%$ $(\mathrm{p}<0.05$, a relative decrease of $53 \pm 26 \%)$, respectively. Although mean arterial pressure and stroke volume index tended to increase ( $41 \pm 6$ versus $45 \pm 4 \mathrm{ml} / \mathrm{min} / \mathrm{m} 2, \mathrm{p}=0.14$ and $41 \pm 6$ versus $45 \pm 4$ $\mathrm{ml} / \mathrm{min} / \mathrm{m} 2, \mathrm{p}=0.08$ ), and heart rate tended to decrease ( $73 \pm 15$ versus $68 \pm 111 / \mathrm{min}, 0.05$ ), no significant change was found. No significant change was found in respiratory parameter (tidal volume, res- 
piratory rate or inspiratory pressure; $\mathrm{p}=0.36,0.34$ and 0.17 , respectively) or bladder pressure $(6.0 \pm 3.7$ versus $5.6 \pm 2.7 \mathrm{mmHg}, \mathrm{p}=0.6$ ) either.

Conclusions. Opening of the abdomen decreases PPV and SVV. During goal directed therapy, current thresholds for fluid responsiveness should be changed accordingly. 


\subsection{Introduction}

Optimizing cardiac stroke volume during major surgery decreases the incidence of postoperative complications and the length of stay in the ICU [1-3]. Dynamic preload indicators, like stroke volume variation (SVV) and pulse pressure variation (PPV), are reliable predictors of fluid responsiveness during mechanical ventilation [4]. Therefore, it has been suggested that these indicators should be optimized during major surgery by goal directed fluid therapy [5]. Dynamic preload indicators are the result of the concerted effect of the swings in intrathoracic pressure during mechanical ventilation [6]. The extent to which positive pressure ventilation increases intrathoracic pressure and interacts with the intrathoracic circulation depends on the tidal volume and the compliance of the chest wall [7]. Because the diaphragm is part of the thoracic cavity, the resistance of the diaphragm to change shape, contributes to the chest wall compliance. In turn, this resistance of the diaphragm is influenced by the abdominal pressure ( $\mathrm{Pab}$ ), which acts as an opposing force to the diaphragmatic descent during mechanical inspiration. An increase in Pab therefore results in decreased chest wall compliance and more pronounced arterial pressure variations [8]. For this reason, the use of higher threshold values has been suggested for the prediction of fluid responsiveness in patients with increased Pab [9]. However, the influence of a decreased abdominal pressure on dynamic preload indicators is unknown. We hypothesized that by opening of the abdominal compartment, the chest wall compliance will increase and dynamic preload indicators will be decreased in magnitude. This would imply that currently used threshold values for the prediction of fluid responsiveness are not applicable during open abdomen surgical procedures. The aim of this study was therefore to assess the effects of opening of the abdominal cavity on dynamic preload indicators.

\subsection{Methods}

\subsubsection{Patients}

Thirteen patients on controlled mechanical ventilation were studied during elective abdominal surgery requiring laparotomy. The study has been carried out in the Netherlands in accordance with the applicable rules concerning the review of research ethics committees and informed consent. Exclusion criteria consisted of a Body Mass Index (BMI) $>35 \mathrm{~kg} / \mathrm{m} 2$, recent $(<2$ months) bladder surgery or trauma and any cardiac arrhythmias. Patients were also excluded when administration of epidural anaesthetics or fluid resuscitation was needed to maintain hemodynamic stability during the execution of the study protocol. 


\subsubsection{Physiological monitoring}

Pressure monitoring included non-invasive arterial blood pressure (ABP) and bladder pressure as a measure of abdominal pressure (Pab). The ABP was measured continuously using an inflatable finger cuff in combination with the Nexfin Monitor (BMEYE, Amsterdam, The Netherlands) [10]. After insertion of the urinary catheter the empty bladder was filled with $25 \mathrm{ml}$ of $0.9 \% \mathrm{NaCl}$ solution (Baxter BV, Utrecht, The Netherlands) and subsequently connected to a pressure monitoring set (Edwards Lifesciences LLC, Irvine, California, USA) [11]. Both blood pressure and bladder pressure were both recorded on a laptop computer with a sample rate of $200 \mathrm{~Hz}$ using an A/D converter (NI USB-6211, National Instruments, Austin, Texas, USA). Cardiac index (CI) and stroke volume index (SVI) were calculated from the ABP using the pulse contour method incorporated in the Nexfin Monitor (BMEYE, Amsterdam, The Netherlands) $[12,13]$. Pulse pressure variation (PPV) and stroke volume variation (SVV) were calculated offline using Matlab (Matlab R2012a, Mathworks Inc., Natick, MA, USA), according to equation 1 and 2, respectively:

PPV $(\%)=100 *[($ PPmax $-P P \min ) /[(\mathrm{PPmax}+\mathrm{PPmin}) / 2]$

Eq. 1

$\operatorname{SVV}(\%)=100 *[($ SVImax-SVImin $) /[($ SVImax+SVImin $) / 2] \quad$ Eq. 2,

in which PPmax/SVImax and PPmin/SVImin were the maximum and minimum pulse pressure/stroke volume index over one breath, and subsequently averaged over 5 consecutive respiratory cycles [14].

\subsubsection{Study protocol}

Recording of physiological data started after anaesthetic induction. The urinary catheter was clamped shortly after insertion after all urine had drained from the bladder. After clamping the catheter, a $21 \mathrm{G}$ needle connected to a pressure monitoring set was inserted and secured in the entry point of the catheter. Recording of the abdominal pressure started one minute after the priming of the bladder by injecting $25 \mathrm{~mL}$ of $0.9 \% \mathrm{NaCl}$. ABP, Pab and respiratory parameters were recorded for one minute just before opening of the abdominal compartment and one minute immediately after. Patients were ventilated with (pressure regulated) volume-controlled ventilation in order to maintain the same tidal volume before and after opening of the abdomen since tidal volume is the main determinant of changes in intrathoracic pressure.

\subsubsection{Anaesthetics}

Anaesthesia was induced with propofol 1-2.5 mg kg-1 and sufentanil $10-50 \mu \mathrm{g}$. Endotracheal intubation was facilitated with neuromuscular blockade established with rocuronium $0.6-1.0 \mathrm{mg} \mathrm{kg}-1$. Anaesthesia was maintained with isoflurane $0.6-1.1 \%$ or sevoflurane 1.1-1.6\% applied with a mixture of air and oxygen. When an epidural catheter was placed, the position was verified with a test dose of $3 \mathrm{ml}$ lidocaine $2 \%$ 
with $5 \mu \mathrm{g}$ ml-1 epinephrine. Further epidural local anaesthetics were not administered until after the measurements.

\subsubsection{Statistical analysis}

Sharipo-Wilk tests were performed to verify a Gaussian distribution of the data. All results are displayed as mean and standard deviation (SD), unless stated otherwise. A paired student t-test is performed to determine the statistical significance of the change in parameters before and after opening of the abdomen. Statistical analysis was performed using GraphPad Prism version 5.00 for Windows (GraphPad, San Diego, CA, USA), and a two-sided p-value of $<0.05$ was considered significant.

\subsection{Results}

The study population consisted of thirteen patients. In three patients SVV could not be determined due to technical difficulties. All data were normally distributed. Patient characteristics are presented in Table 1.

Table 1: Patients characteristics. VCV= Volume Controlled Ventilation, $P R V C=$ Pressure Regulated Volume Controlled. Data expressed as mean $\pm S D$.

\begin{tabular}{||l|l||}
\hline \multicolumn{2}{||c||}{ patient characteristics } \\
\hline Patient $(\#)$ & 13 \\
Male/Female $(\#)$ & $6 / 7$ \\
Age $(\mathrm{yr})$ & $54 \pm 13$ \\
Weight $(\mathrm{kg})$ & $81 \pm 16$ \\
BMI $\left(\mathrm{kg} \cdot \mathrm{m}^{-2}\right)$ & $27 \pm 4$ \\
Ventilatory mode & Volume controlled mode \\
\hline
\end{tabular}

Table 2 shows the ventilatory parameters (tidal volume $[\mathrm{ml} / \mathrm{kg}$ predicted body weight]), respiratory rate, peak pressure and positive end expiratory pressure) before and after opening of the abdomen. No significant differences were found as a result of the opening of the abdomen. Although heart rate, mean arterial pressure, SVI or CI tended to change, no significant differences were found (see Figure 1). There were also no relevant changes in infusion rate of anaesthetics (Sevoflurane, Isoflurane, Sufentanil, Ephidrine or Phenylephrine). As a result of the opening of the abdomen, the PPV significantly $(\mathrm{p}<0.005)$ decreased from $11.55 .8 \%$ to $6.42 .9 \%$, a relative decrease of $4019 \%$, see Figure 2 . The SVV also decreased significantly $(\mathrm{p}<0.05)$ from $12.76 .1 \%$ to $4.81 .6 \%$ (a relative decrease of $53 \pm 26 \%)$. Opening of the abdomen did not significantly change mean bladder pressure (mean pressure of $6.0 \pm 3.7$ versus $5.6 \pm 2.7 \mathrm{mmHg}, \mathrm{p}=0.6$ ). 

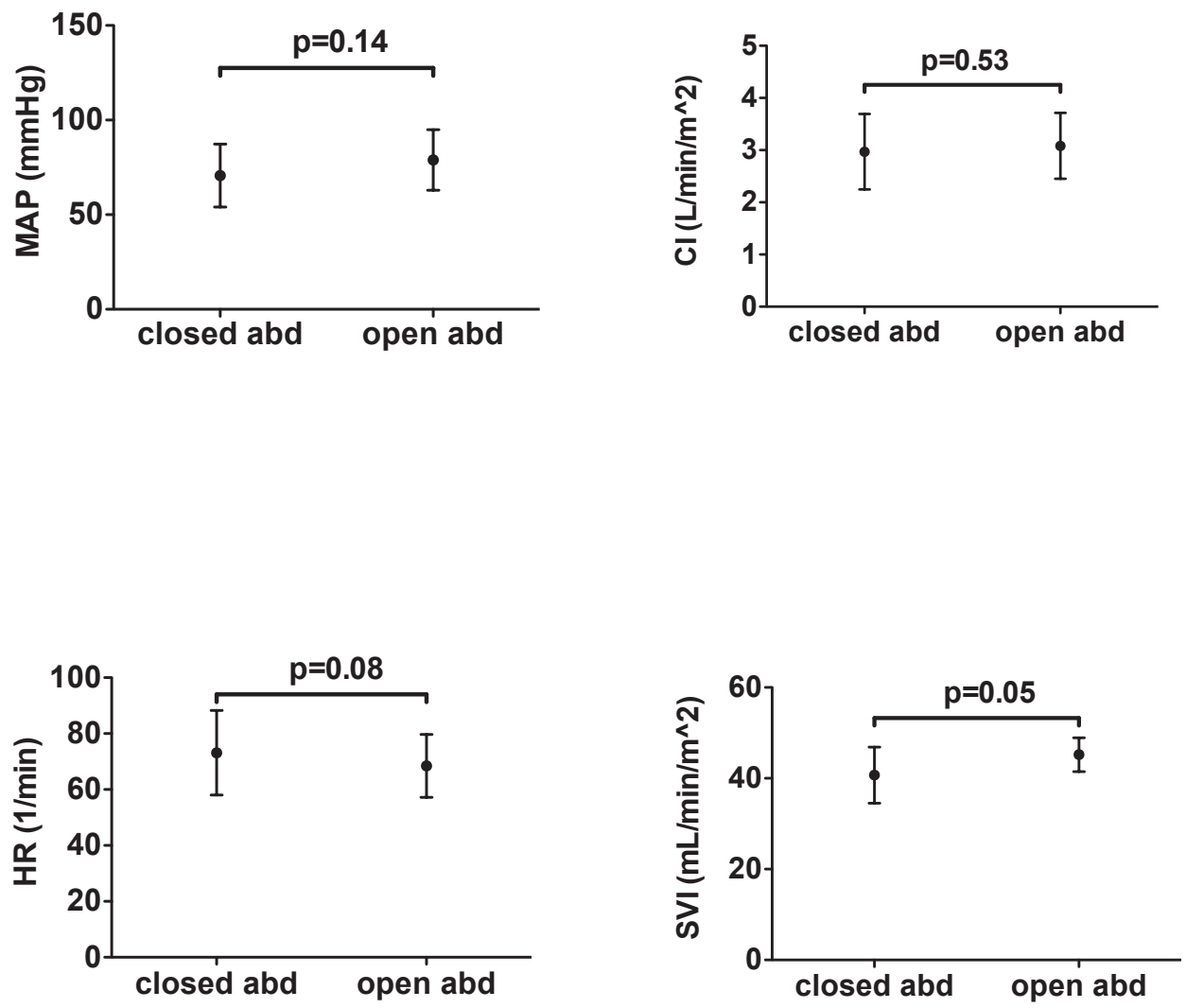

Figure 1: Comparison of main hemodynamic variables during a closed abdomen and opened abdomen. $M A P=$ mean arterial pressure, $C I=$ Cardiac Index, $H R=$ heart rate, $S V I=$ Stroke Volume Index, p-values were not significant and mentioned in the figure. 

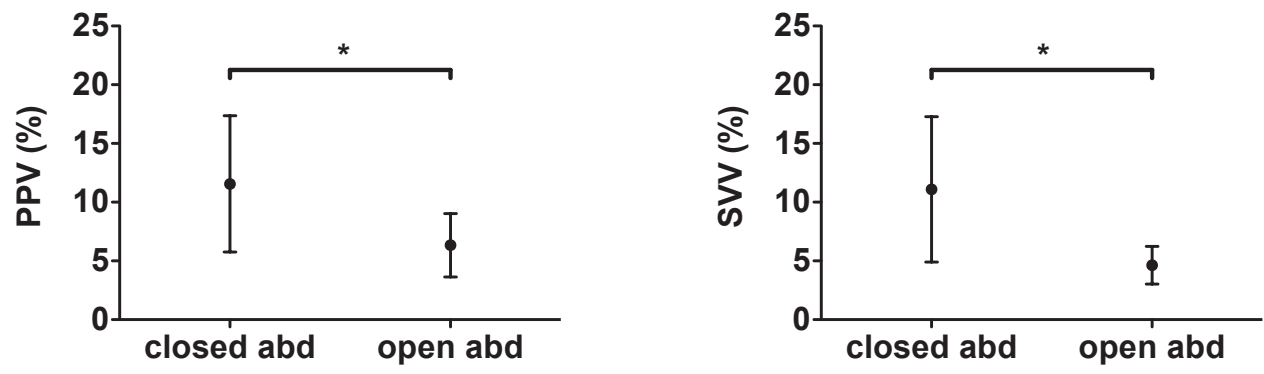

Figure 2: Comparison of waveform derived preload indicators at closed abdomen and at opened abdomen. $P P V=$ pulse pressure variation, $S V V=$ stroke volume variation, $*=p$ $<0.01$.

Table 2: Ventilatory parameters just before and after opening of the abdomen. PEEP= positive end expiratory pressure. Data expressed as mean $\pm S D$.

\begin{tabular}{||l|l|l|l||}
\hline & Closed abdomen & Open abdomen & P-value \\
\hline Respiratory rate $\left(1 \cdot \mathrm{min}^{-1}\right)$ & $12.1 \pm 1.0$ & $12.1 \pm 1.0$ & 0.34 \\
Tidal volume $\left(\mathrm{ml} \cdot \mathrm{kg}^{-1}\right)$ & $7.6 \pm 1.2$ & $7.3 \pm 0.7$ & 0.36 \\
PEEP $\left(\mathrm{cmH}_{2} \mathrm{O}\right)$ & $4.5 \pm 1.7$ & $4.7 \pm 1.5$ & 0.32 \\
Peak inspiratory pressure $\left(\mathrm{cmH}_{2} \mathrm{O}\right)$ & $16.6 \pm 2.6$ & $15.9 \pm 2.6$ & 0.17 \\
\hline
\end{tabular}

\subsection{Discussion}

This study shows that the magnitude of both PPV and SVV decrease upon opening the abdomen, emphasizing that caution is advised when using these dynamic preload indicators for goal directed fluid therapy during open abdominal surgery.

Dynamic preload indicators accurately predict fluid responsiveness under stable and controlled physiological conditions (e.g. tidal volume $>8 \mathrm{ml} / \mathrm{kg}$, no arrhythmias and HR to ventilator ratio $>3.6$ ), however when applied under ambiguous physiological conditions their predictive value deteriorates [15]. Hence, when applying these indicators in a surgical setting cautiousness is mandated by the fact that patients physiology may be affected by administered drugs, anesthesia and surgery. This vigilance required during the application of dynamic preload indicators in the setting of 
surgery is increasingly recognized, and together with our findings, emphasizes the drawbacks of dynamic preload indicators in clinical practice [16]. When abdominal pressure increases, e.g. due to pneumoperitoneum, dynamic preload indicators increase, subsequently decreasing their predictive accuracy if the currently proposed threshold values would be applied $[8,9]$. While several studies have suggested that dynamic preload indicators are still able to predict fluid responsiveness during abdominal surgery to a certain extent $[17,18]$, our findings confirms the results of other studies that their predictive value regarding fluid responsiveness is reduced compared to values obtained on the ICU $[4,19,20]$.

Since the tidal volume, the main determinant for the change in intrathoracic pressure, 21 did not change, the observed decrease of the dynamic preload indicators must be a result of the decreased swings in pleural pressure due to the increase in thoracic compliance (or more specific, the increased compliance of the diaphragm as a result of the increased abdominal compliance). This increase in thoracic compliance however, did not result in a significant decrease in inspiratory pressure. This is explained by the fact that the main determinant of the airway pressure, lung compliance, did not change in our patients that did not suffer from any serious illness and had no apparent chest wall edema. Our finding that also the bladder pressure was not decreased is likely related to patients being under general anesthesia, and having received muscle relaxation, when the abdominal pressure is mainly determined by the gravity component of the above-lying structures, and not the compliance of the abdominal compartment. This is also confirmed by the fact that the swings in bladder pressure due to mechanical ventilation were only $0.6 \pm 0.2 \mathrm{mmHg}$ during closed abdomen, and that this change was similar in open abdominal situation $(0.6 \pm 0.3 \mathrm{mmHg}, \mathrm{p}=0.74)$. Another reason might be that we used an indirect method to measure abdominal pressure instead of a direct method. Although the used method is considered the gold standard [11], there are some concerns regarding the reliability and reproducibility of this indirect method [21]. Also urine output in between the two measurements could be a reason for the bladder pressure not to decrease. However, since the time in between the two measurements was only around 15 minutes, the influence of this effect was probably small.

This study has several limitations that need to be addressed. First, the arterial pressure was not measured by an invasive arterial catheter, but non-invasively using a finger cuff. As this method has an excellent correlation with invasively recorded arterial pressure and the derived dynamic preload predictors [13], it is unlikely that this impairs the validity of our results. Furthermore, several patients received an epidural catheter prior to the surgery and location was verified with a test dose of $3 \mathrm{ml}$ lidocaine $2 \%$ with $5 \mu \mathrm{g}$ ml-1 epinephrine. Due to the low dose of lidocaine administered and the time interval until the measurements, the test dose was assumed not to influence the measurements $[22,23]$. We also assumed that vasoactive drugs, ad- 
ministered during surgery, did not influence the dynamic preload indicators, however studies on this subject are scarce [24, 25]. To obviate the prerequisite of hemodynamic equivalence before and after opening of the abdominal compartment, both blood pressure and cardiac index between measurements were compared and found to be similar, legitimating the assumption of an unchanged hemodynamic status in between measurements.

In conclusion, in this study we have assessed the effects of opening of the abdominal cavity on dynamic preload indicators. A significant decrease in arterial waveform derived dynamic variables, SVV and PPV, was found after opening the abdominal compartment, indicating an increased risk of false negative predictions for fluid responsiveness if unchanged thresholds would be applied.

\section{References}

[1] T. Corcoran, J. E. Rhodes, S. Clarke, P. S. Myles, and K. M. Ho, "Perioperative fluid management strategies in major surgery: a stratified meta-analysis," Anesthesia and analgesia, vol. 114, no. 3, pp. 640-51, 2012.

[2] L. Dalfino, M. T. Giglio, F. Puntillo, M. Marucci, and N. Brienza, "Haemodynamic goal-directed therapy and postoperative infections: earlier is better. a systematic review and meta-analysis," Critical care, vol. 15, no. 3, p. R154, 2011.

[3] M. Y. Kirov, V. V. Kuzkov, and Z. Molnar, "Perioperative haemodynamic therapy," Current opinion in critical care, vol. 16, no. 4, pp. 384-92, 2010.

[4] P. E. Marik, X. Monnet, and J. L. Teboul, "Hemodynamic parameters to guide fluid therapy," Annals of intensive care, vol. 1, no. 1, p. 1, 2011.

[5] M. A. Hamilton, M. Cecconi, and A. Rhodes, "A systematic review and metaanalysis on the use of preemptive hemodynamic intervention to improve postoperative outcomes in moderate and high-risk surgical patients," Anesthesia and analgesia, vol. 112, no. 6, pp. 1392-402, 2011.

[6] F. Michard, "Changes in arterial pressure during mechanical ventilation," Anesthesiology, vol. 103, no. 2, pp. 419-28, 2005.

[7] R. Novak, G. M. Matuschak, and M. R. Pinsky, "Effect of positive-pressure ventilatory frequency on regional pleural pressure," Journal of applied physiology, vol. 65, no. 3, pp. 1314-23, 1988. 
[8] D. Jacques, K. Bendjelid, S. Duperret, J. Colling, V. Piriou, and J. P. Viale, "Pulse pressure variation and stroke volume variation during increased intraabdominal pressure: an experimental study," Critical care, vol. 15, no. 1, p. R33, 2011.

[9] B. Tavernier and E. Robin, "Assessment of fluid responsiveness during increased intra-abdominal pressure: keep the indices, but change the thresholds," Critical care, vol. 15, no. 2, p. 134, 2011.

[10] D. W. Eeftinck Schattenkerk, J. J. van Lieshout, A. H. van den Meiracker, K. R. Wesseling, S. Blanc, W. Wieling, G. A. van Montfrans, J. J. Settels, K. H. Wesseling, and B. E. Westerhof, "Nexfin noninvasive continuous blood pressure validated against riva-rocci/korotkoff," Am J Hypertens, vol. 22, no. 4, pp. 378 83, 2009.

[11] M. L. Cheatham, M. L. Malbrain, A. Kirkpatrick, M. Sugrue, M. Parr, J. De Waele, Z. Balogh, A. Leppaniemi, C. Olvera, R. Ivatury, S. D’Amours, J. Wendon, K. Hillman, and A. Wilmer, "Results from the international conference of experts on intra-abdominal hypertension and abdominal compartment syndrome. ii. recommendations," Intensive care medicine, vol. 33, no. 6, pp. 951-62, 2007.

[12] K. H. Wesseling, N. smith, W. Nichols, H. Weber, B. de Wit, and J. E. Beneken, Beat-to-beat cardiac output from the arterial pressure pulse contour, ser. Boerhaave series for postgraduate medical education. Leiden: Leiden University Press, 1974, pp. 148-64.

[13] B. Lansdorp, D. Ouweneel, A. de Keijzer, J. G. van der Hoeven, J. Lemson, and P. Pickkers, "Non-invasive measurement of pulse pressure variation and systolic pressure variation using a finger cuff corresponds with intra-arterial measurement," British journal of anaesthesia, vol. 107, no. 4, pp. 540-5, 2011.

[14] H. K. Kim and M. R. Pinsky, "Effect of tidal volume, sampling duration, and cardiac contractility on pulse pressure and stroke volume variation during positive-pressure ventilation," Crit Care Med, vol. 36, no. 10, pp. 2858-62, 2008.

[15] B. Lansdorp, J. Lemson, M. J. van Putten, A. de Keijzer, J. G. van der Hoeven, and P. Pickkers, "Dynamic indices do not predict volume responsiveness in routine clinical practice," British journal of anaesthesia, vol. 108, no. 3, pp. 395-401, 2012.

[16] S. Y. Yang, J. K. Shim, Y. Song, S. J. Seo, and Y. L. Kwak, "Validation of pulse pressure variation and corrected flow time as predictors of fluid responsiveness 
in patients in the prone position," British journal of anaesthesia, vol. 110, no. 5, pp. 713-20, 2013.

[17] A. Derichard, E. Robin, B. Tavernier, M. Costecalde, M. Fleyfel, J. Onimus, G. Lebuffe, J. P. Chambon, and B. Vallet, "Automated pulse pressure and stroke volume variations from radial artery: evaluation during major abdominal surgery," Br J Anaesth, vol. 103, no. 5, pp. 678-84, 2009.

[18] J. Y. Lee, H. Y. Park, W. S. Jung, Y. Y. Jo, and H. J. Kwak, "Comparative study of pressure- and volume-controlled ventilation on stroke volume variation as a predictor of fluid responsiveness in patients undergoing major abdominal surgery," Journal of critical care, vol. 27, no. 5, pp. 531 e9-14, 2012.

[19] L. O. Hoiseth, I. E. Hoff, O. Skare, K. A. Kirkeboen, and S. A. Landsverk, "Photoplethysmographic and pulse pressure variations during abdominal surgery," Acta anaesthesiologica Scandinavica, vol. 55, no. 10, pp. 1221-30, 2011.

[20] G. Gouvea, R. Diaz, L. Auler, R. Toledo, and J. M. Martinho, "Evaluation of the pulse pressure variation index as a predictor of fluid responsiveness during orthotopic liver transplantation," Br J Anaesth, vol. 103, no. 2, pp. 238-43, 2009.

[21] M. L. Malbrain, "Different techniques to measure intra-abdominal pressure (iap): time for a critical re-appraisal," Intensive care medicine, vol. 30, no. 3, pp. 357-71, 2004.

[22] W. A. Visser, T. H. Liem, J. van Egmond, and M. J. Gielen, "Extension of sensory blockade after thoracic epidural administration of a test dose of lidocaine at three different levels," Anesthesia and analgesia, vol. 86, no. 2, pp. 332-5, 1998.

[23] S. J. Holman, R. R. Bosco, T. C. Kao, M. A. Mazzilli, K. J. Dietrich, R. A. Rolain, and R. A. Stevens, "What constitutes an effective but safe initial dose of lidocaine to test a thoracic epidural catheter?" Anesthesia and analgesia, vol. 93, no. 3, pp. 749-54, 2001.

[24] M. Hadian, D. A. Severyn, and M. R. Pinsky, "The effects of vasoactive drugs on pulse pressure and stroke volume variation in postoperative ventilated patients," Journal of critical care, vol. 26, no. 3, pp. 328 e1-8, 2011.

[25] M. Cannesson, Z. Jian, G. Chen, T. Q. Vu, and F. Hatib, "Effects of phenylephrine on cardiac output and venous return depend on the position of the heart on the frank-starling relationship," Journal of applied physiology, vol. 113, no. 2, pp. 281-9, 2012. 


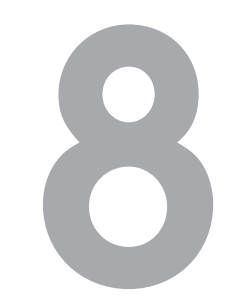

\title{
A mathematical model for the prediction of fluid responsiveness
}

\author{
B. Lansdorp, M.J.A.M. van Putten, A. de Keijzer, P. Pickkers, J. van Oostrom. \\ Cardiovascular Engineering and Technology 2013 March;4(1):53-62
}

Fluid therapy is commonly used to improve cardiac output in hemodynamically instable patients in the intensive care unit. However, to predict whether patients will benefit from this intervention (i.e. are volume responsive), is difficult. Dynamic indices, that rely on heart-lung interactions, have shown to be good predictors of fluid responsiveness under strict clinical conditions, but clinical use is still limited. This is due to the lack of understanding of the complex underlying physiology since multiple quantities are involved. We present a physiologically based mathematical model of the interaction between the respiratory and cardiovascular systems incorporating dynamic indices and fluid responsiveness. Our model is based on existing models of the cardiovascular system, its control, and the respiratory system during mechanical ventilation. The model of the cardiovascular system is expanded by including nonlinear cardiac elastances to improve simulation of the Frank-Starling mechanism. An original model including five mechanisms for interaction between mechanical ventilation and the circulation is also presented. This model allows for the simulation of these complex relationships and may predict the effect of volume infusion in specific patients in the future. The presented model must be seen as a first step to a bedside clinical decision support system, and can be used as an educational model. 


\subsection{Introduction}

Volume resuscitation is one of the most common therapeutic procedures in intensive care units to improve cardiac output (CO) or stroke volume index (SVI) and thus hemodynamics in critically ill patients. However, to identify patients who might benefit from this therapy by an increase in cardiac output (volume responders), is a clinical challenge. Both clinical examination and static indicators of cardiac preload (e.g. central venous pressure) have been shown to be of minimal predictive value in distinguishing volume responders from non-responders [1,2]. Over the last decade, dynamic indices that rely on cardiopulmonary interactions are used to assess fluid responsiveness in ventilated patients. Examples of dynamic indices are pressure or flow fluctuations that can be observed within the peripheral arteries. They are caused by mechanical ventilation when the heart operates on the steep portion of the FrankStarling curve instead of on the flat portion of the curve and changes in preload cause variations in stroke volume [3, 4].

Although dynamic indices have shown to be good predictors of volume responsiveness $[1,5]$, their use is limited to selected populations of patients and requires specific conditions for its application [6]. The reason for this limited applicability is the complex underlying physiology in which many quantities are involved (e.g. tidal volume, lung compliance, chest wall compliance and volume status), which makes the dynamic indices difficult to interpret. It is our contention that by a better understanding of the complex relationships between the involved quantities, this use can be expanded. It is our goal to develop a mathematical model that captures the dynamics of heart-lung interactions and their relation to a patient's volume status. Such a model, that is able to simulate the highly interrelated processes of this complex physiology, could also be used for educational simulations [7-10]. When adaptable to the individual patient, the model could form the basis of a decision support system by predicting the effect of any considered volume infusion in specific patients [11-13].

A first, and to our knowledge only step towards the identification of factors that influence the arterial pressure variations by cardiovascular modeling was made by Messerges [14]. He endorsed the assumption that mathematical modeling potentially lead to more clinically relevant interpretation of dynamic indices, and introduced positive pressure ventilation, venous compression and a rightward septum shift into an existing cardiovascular model. Unfortunately, this study was not able to link dynamic indices to volume status, probably because of the use of a cardiac model with limited complexity, the absence of respiratory mechanics and an incomplete description of the interacting mechanisms. Studies related to the life-science space program also modeled external pressure influences on the cardiovascular system [15]. Although they make use of similar concepts relevant for modeling heart-lung interaction and volume responsiveness, including blood volume shifts, baroreflex models, and changes in venous transmural pressure, they are intended to be used to investigate the 
effects of postspaceflight orthostatic intolerance so lack respiratory mechanics and specific heart-lung interactions.

The primary objective of the work presented in this paper is to develop and test a physiological model of cardiovascular function that contains the essential processes associated with the prediction of fluid responsiveness. In particular, we report on the extension of previously developed and validated models of the cardiovascular system, which were initially developed for educational purposes. In this paper, we extend these models with several aspects of the cardiovascular and respiratory system, which are relevant during mechanical ventilation and in the dynamics of the heart-lung interactions in order to be able to simulate the relation between patient characteristics, volume status and dynamic indices in mechanically ventilated patients. We will use model parameters based on literature values as much as possible. Newly introduced parameters and constants will be chosen to simulate experimental data in the best way possible and listed in Table A in Applendix B. For validation, the simulation results will be compared to clinical data reported in literature. Furthermore, a case study, based on two patients from the ICU of the Radboud University Medical Center Nijmegen will be presented to show how the model is intended to be used in the future as a decision support system to prevent patients from receiving unnessecary, and possibly harmfull fluids.

\subsection{Model Description}

\subsubsection{Cardiovascular system}

Our model is based on previously described models of the closed circulation and consist of multiple segments that are lumped together [16-19]. In short, four compartments describe the heart, six vascular compartments describe the systemic circulation and three vascular compartments describe the pulmonary circulation. In the previously described models, the systemic circulation consisted of five compartments, however, we have split the intrathoracic artery compartment into an aorta and a new intrathoracic compartment. This was done in order to make it possible to differentiate between characteristics of the ascending part of the aorta and those of the more distal part of the intrathoracic arteries. Furthermore, four valves prevent backflow of blood from the ventricles into the atria during systole and from the arteries into the ventricles during diastole. The model is represented in Figure 1.

The relation between the pressure $(p)$, volume $(v)$, and flow $(f)$ within the cardiac and vascular compartments is described by three equations. Equation 1 results from conservation of volume, equation 2 from the hydraulic equivalent of the Kirchhoff's voltage law and the descriptions of the inertance $(L)$ and resistance $(R)$ components, 


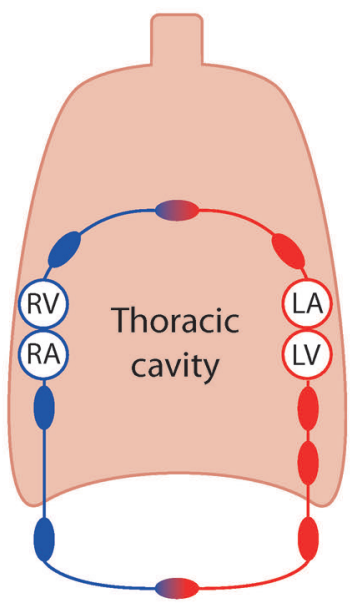

Figure 1: representation of the used closed, uncontrolled cardiovascular model with illustrated $R A, R V, L A$ and $L V$ being right atrium, right ventricle, left atrium and left ventricle together with the systemic and pulmonary arteries, capillarries and veins.

and equation 3 describes the compliance $(C)$ component:

$$
\begin{array}{rll}
\delta v(t) \cdot \delta t^{-1} & =f_{\text {in }}(t)-f_{\text {out }}(t) & \\
\delta f_{\text {in }}(t) \cdot \delta t^{-1} & =1 \cdot L^{-1} \cdot\left(p_{\text {in }}(t)-p_{\text {out }}(t)-R \cdot f_{\text {in }}(t)\right) \\
p_{\text {out }}(t) & =1 \cdot C^{-1} \cdot\left(v(t)-V_{u}\right) & \text { if } v(t)>V_{u} \\
& =0 & \text { else. }
\end{array}
$$

The inertance $(L)$ can be negleted in many compartments since the change in blood flow over time is small, and/or the vessels cross sectional area is so big that the inductance is negligibly small, simplifying equation 2 to a static one. The unstressed volume $\left(V_{u}\right)$ represents the intra-compartment volume where the transmural pressure is zero. The electrical equivalent of a generic compartment within the circulation model is shown in Figure A in Appendix A.

The four heart compartments are modeled as elastic chambers with contractile characteristics. Switching the heart between systolic and diastolic function $\left(e_{\text {diast }}\right.$ and $e_{\text {syst }}$, respectively, with $e=1 / C$ and $e_{\text {syst }}$ is a time-vaying elastance), generates the pulsatile flow, as described earlier [16]:

$$
\begin{aligned}
e_{\text {diast }}(t) & =e_{\text {min }} \\
e_{\text {syst }}(t) & =e_{\text {min }}+\left(e_{\text {max }}-e_{\text {min }}\right) \cdot \sin \left(\pi \cdot \frac{t_{c c}-T_{d}}{T_{s}}\right),
\end{aligned}
$$


where $t_{c c}$ is the time in the current heart cycle and $T_{d}$ and $T_{s}$ are the duration of the chamber's diastole and systole, respectively.

The cardiac elastances however, are also are a function of the blood volume within the chamber (see Figure 2). During diastole, while the volume of the chamber $\left(v_{\text {chamber }}\right)$ is below its unstressed volume multiplied by $k$ (e.g. at $V_{1}$ in Figure 2), diastolic elastance is constant $\left(e_{\min }(t)\right)$. Above this value (e.g. at $V_{2}$ in Figure 2), fibrous tissue will linearly increase the chambers elastance until $v_{\text {chamber }}$ reaches its unstressed volume multiplied by $m$ :

$$
\begin{array}{r}
e_{\text {min }}(t)=e_{\text {min }} \quad \text { for } v_{\text {chamber }}(t) \leq k \cdot V_{u} \\
e_{\text {min }}(t)=e_{\text {min }}+\left(\Delta e_{\text {diast }}^{\max }\left(\frac{v_{\text {chamber }}(t)-k \cdot V_{u}}{m \cdot V_{u}-k \cdot V_{u}}\right)\right) \\
\quad \text { for } m \cdot V_{u} \geq v_{\text {chamber }}(t)>k \cdot V_{u},
\end{array}
$$

where $\Delta e_{\text {diast }}^{\max }$ is the maximum increase in diastolic elastance due to the pericardium and $k$ and $m$ are constants. The maximum elastance during systole $\left(e_{\max }(t)\right)$, will follow the Frank-Starling mechanism [20]. In essence, this means that (in accordance with the sliding filament theory [21]) the more the cardiac muscle is stretched, the larger the force of contraction is, until the muscle reaches its optimal length at $v_{\text {chamber }}(t)=k \cdot V_{u}$. Exceeding this optimal length will reduce systolic elastance (e.g. at $V_{2}$ in Figure 2), represented by a linear reduction in elastance, until the systolic elastance theoretically equals the diastolic elastance at $v_{\text {chamber }}(t)=m \cdot V_{u}$ :

$$
\begin{array}{r}
e_{\max }(t)=e_{\max } \quad \text { for } v_{\text {chamber }}(t) \leq k \cdot V_{u} \\
e_{\max }(t)=e_{\max }-\left(\left(e_{\max }-e_{\min }\right) \cdot\left(\frac{v_{\text {chamber }}(t)-k \cdot V_{u}}{m \cdot V_{u}-k \cdot V_{u}}\right)\right) \\
\text { for } m \cdot V_{u} \geq v_{\text {chamber }}(t)>k \cdot V_{u} .
\end{array}
$$

In patients operating on the steep part of the systolic elastance curve, the cardiac muscle is not stretched optimally, resulting in a reduction of contraction force. In these patients adding fluid will be beneficial, as the increase in preload will result in an increase in cardiac output.

To be able to realistically simulate the effect of volume expansion, a regulatory set-point model of the arterial baroreflex is implemented. The used model [19] aims at maintaining mean arterial blood pressure constant by dynamically adjusting heart rate $(h r)$, heart contractility (contr), systemic vascular resistance (svr) and the unstressed volume of the venous system $\left(v_{0, v e n}\right)$. In this model, the inhibitory/stimulatory activity of the baroreceptor $\left(b r_{a c t}\right)$ depends linearly on the difference in mean arterial pressure (map) and the set-point value of the map $\left(M A P_{s p}\right)$ monitored by the baroreceptors. Since the model was developed to simulate a re- 


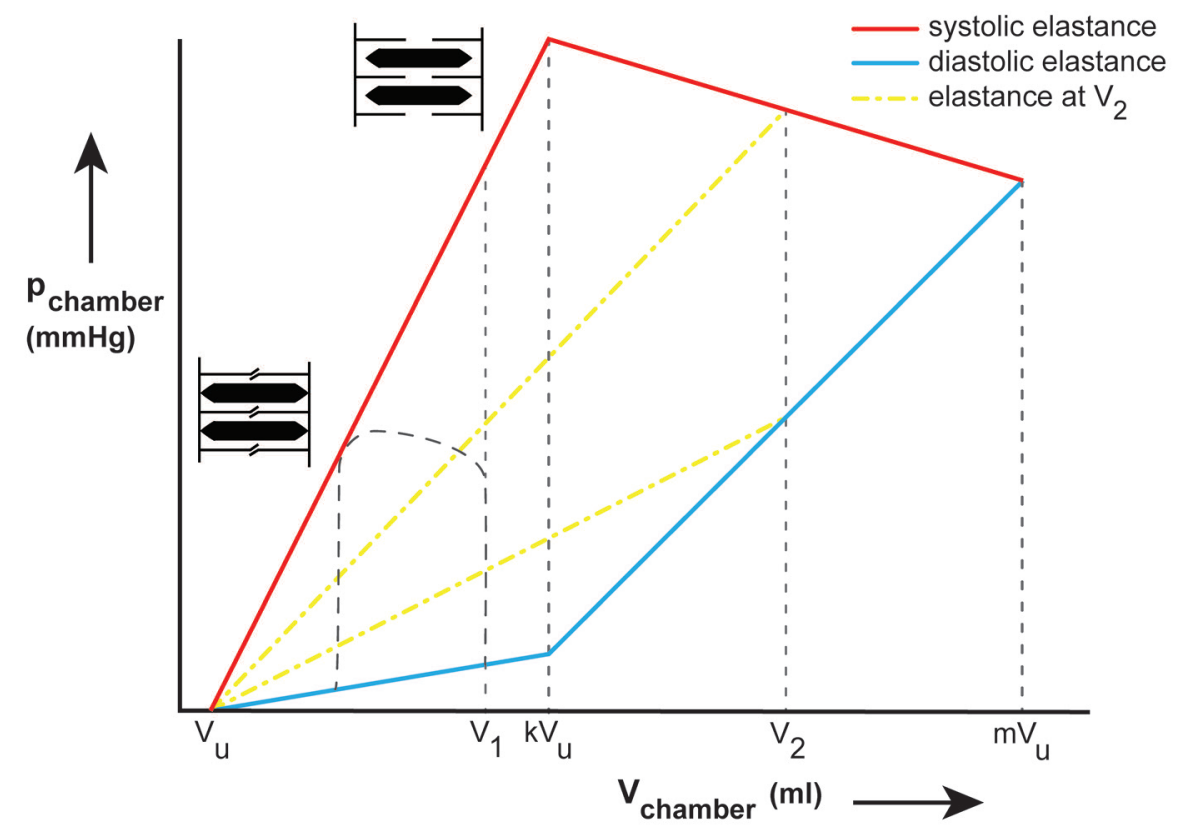

Figure 2: The diastolic and systolic elastance curves illustrate the behaviour of cardiac function $\left(e_{\min }\right.$ and $e_{\max }$ ) as a function of cardiac volume during diastole and systole. Switching the heart between systolic and diastolic function generates the characteristic pressurevolume-loop as shown. The more the cardiac muscle is stretched during diastole, the larger the force of contraction is during systole, until the muscle reaches its optimal length at $v_{\text {chamber }}(t)=k \cdot V_{u}$ (Frank-Starling mechanism). Exceeding this optimal length will increase diastolic elastance and reduce systolic elastance (e.g. at $V_{2}$ in Figure 2) until the systolic elastance theoretically equals the diastolic elastance. 
alistic response to volume status and volume therapy, but not to study beat-to-beat variability, delays and time constants were not included, so the equations are:

$$
\begin{aligned}
b r_{a c t} & =B R_{\min }-M A P_{s p} & & \text { if map }<B R_{\min } \\
& =B R_{\max }-M A P_{s p} & & \text { if map }>B R_{\max } \\
& =\text { map }-M A P_{s p} & & \text { otherwise, }
\end{aligned}
$$

with $B R_{\min }$ and $B R_{\max }$ the thresholds with maximal/minimal baroreceptor activity, respectively. This baroreceptor activity is multiplied by the gain from map to the regulated variable (change per $\mathrm{mmHg}$ deviation in map) and subsequently used to calculate the new value based on the reference value. for example:

$$
h r=\left(1+C_{h r / m a p} \cdot b r_{a c t}\right) \cdot H R_{0} .
$$

The $s v r$, contr and $v_{0, v e n}$ are calculated in a similar way (with different gains and reference values, see appendix B).

\subsubsection{Respiratory system}

The respiratory system during mechanical ventilation is modeled as a lumped system composed of a compliant lung (with compliance $C_{\text {lung }}$ ), an airway (with resistance $R_{a w}$ representing the tube and the bronchial tree) and an additional compliant vessel representing the chest wall (simplified from [22]). Nonlinear flow-dependent resistance to airflow in the upper airways was ignored. The ventilated subject was assumed to be sedated; hence the diaphragm and chest wall were assumed to behave passively with compliance $C_{\text {wall }}$. Air forced into the lungs during positive pressure ventilation $\left(p_{a w}\right)$ performs work on the lung and subsequently the chest wall resulting in a change in pressure across the lung pleura, representing the thorax pressure $p_{t h}$. The relations between lung volume $\left(v_{a w}\right)$, flow $\left(f_{a w}\right)$, airway pressure $\left(p_{a w}\right)$, compliance $(C)$ and resistance $\left(R_{a w}\right)$ can now be expressed as:

$$
\begin{aligned}
\delta v_{\text {aw }}(t) \cdot \delta t^{-1}= & f_{\text {in aw }}(t)-f_{\text {out }, a w}(t) \\
f_{\text {in }, a w}(t)= & \left(p_{\text {aw }}(t)-v_{\text {aw }}(t) .\right. \\
& \left.\left(1 \cdot C_{\text {lung }}^{-1}+1 \cdot C_{\text {wall }}^{-1}\right)\right) \cdot R_{\text {aw }}^{-1} \\
p_{\text {th }}(t)= & 1 \cdot C_{\text {wall }}^{-1} \cdot v_{\text {aw }}(t) .
\end{aligned}
$$

\subsubsection{Heart-lung interaction}

Since the heart, the pulmonary circulation and part of the systemic circulation are located within the thoracic cavity (see Figure 3), they are influenced by changes in in- 


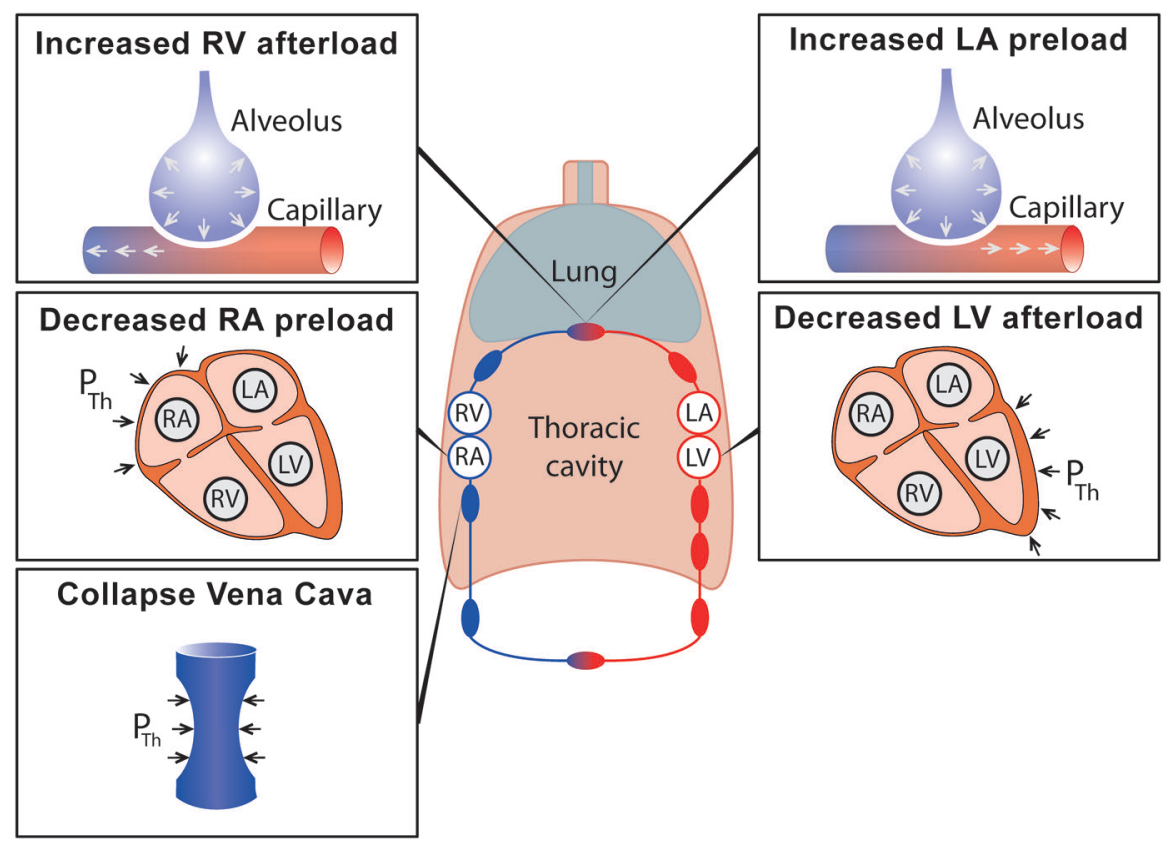

Figure 3: Representation of the combined cardiovascular and respiratory system with the five mechanisms playing a role in the heart-lung interactions. 
trathoracic pressure due to mechanical ventilation. Previously, Michard [3] described five different physiological mechanisms for the effect of intrathoracic pressure on the circulation (see the various panels in Figure 3): collapse of the vena cava (1), increase in intramural pressure in the right atrium (the downstream pressure of the vena cava) (2), compression of the pulmonary capillaries (3 and 4) and intramural pressure of the left ventricle increases (5). These mechanisms are responsible for a decrease in right ventricular preload (mechanisms 1 and 2), increase in right ventricular afterload (3), increase in left ventricular preload (4) and a decrease in left ventricular afterload (5). The increase and decrease in preload results in an increase and decrease in cardiac output, respectively, according to the Frank Starling mechanism as described earlier (Figure 2). The increase and decrease in afterload on the other hand, results in a decrease and increase in cardiac output, respectively, because of the change in driving pressure. This continuous alternation of increase and decrease in cardiac output due to the mechanical ventilation-induced fluctuations in thorax pressure results in typical arterial waveform variations, as illustrated in Figure 4.

The extent to which the described mechanisms are interacting with the circulation is highly depended on the volume status of the patient. In contrast to normo- or hypervolemic patients, hypovolemic patients usually operate on the steep portion of the Starling curve (see Figure 2). Consequently, they will show a relatively large arterial pressure variation due to the changing preload. In addition, due to the relatively low intravascular pressure in hypovolemic patients, the vena cava will collapse and the pulmonary capillaries are compressed during mechanical inspiration.

In the presented model, variation of intra- and trans- mural pressures is modeled by changing the surrounding pressure of the compartments within the thoracic cavity according to the thoracic pressure (see figure 3). This allows the influence on the preload of the right heart and afterload of the left heart (mechanisms 2 and 5 respectively) to be taken into account. The collapsibility of the vena cava (mechanism 1) is simulated by an increase in flow resistance $r_{v c}$ as a function of the pressure difference between the intra- and extra- mural pressure $\left(p_{v c}\right.$ and $\left.p_{t h}\right)$,

$$
r_{v c}=R_{v c, 0}+C_{r, v c} \cdot\left(p_{t h}-p_{v c}\right),
$$

where $R_{v c, 0}$ is the normal resistance of the vena cava and $C_{r, v c}$ a constant.

Compression of the pulmonary capillaries due to an increase in transmural pressure and lung volume, causes an increase in the right ventricular afterload (mechanism 3) and left ventricular preload (mechanisms 4). The effect due to the increase in transmural pressure is modeled by changing the unstressed volume $\left(v_{0, p c}\right)$ proportionally to the transmural pressure (in this case, the difference between the intramural pressure of the capillaries $\left(p_{p c}+p_{t h}\right)$ and the airway pressure $\left.\left(p_{a w}\right)\right)$, see Eq. 8.16. Additionally, the effect caused by the stretching of the capillaries as a result of the increasing lung volume, is modeled by changing the inflow resistance $\left(r_{p c}\right)$ as a func- 


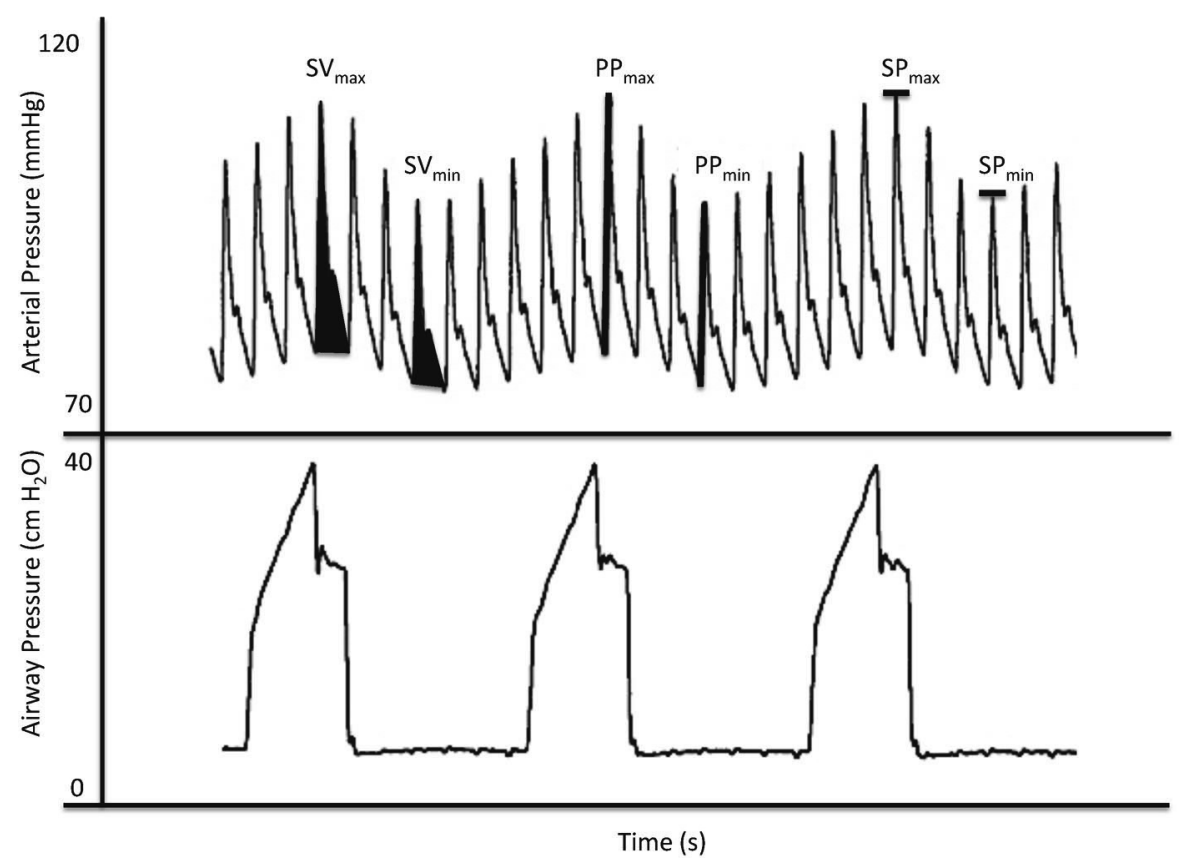

Figure 4: Illustration of the fluctuations in arterial pressure (upper part, with marked maximum and minimum systolic pressure $\left(S P_{\max } \& S P_{\min }\right)$, pulse pressure $\left(P P_{\max } \& P P_{\min }\right)$ and stroke volume $\left(S V_{\max } \& S V_{\min }\right)$ caused by ventilator-induced variations in airway pressure (lower part).

tion of the lung volume $\left(v_{\text {lung }}\right)$, see Eq. 8.17,

$$
\begin{aligned}
v_{0, p c} & =V_{0, p c, 0}-C_{v 0, p c} \cdot\left(p_{a w}-\left(p_{p c}+p_{t h}\right)\right) \\
r_{p c} & =R_{p c, 0}+\log _{10}\left(1+v_{\text {lung }}\right) \cdot C_{r, p c},
\end{aligned}
$$

where $V_{0, p c, 0}$ and $R_{p c, 0}$ are normal values of the unstressed volume and the inflow resistance of the pulmonary capillaries, respectively. $C_{v 0, p c}$ and $C_{r, p c}$ are constants.

\subsubsection{Dynamic indices}

For the quantification of the variation in arterial pressure and stroke volume in the model, reflecting the volume status of the patient, dynamic indices are used which include the pulse pressure variation $(P P V)$, systolic pressure variation $(S P V)$ and stroke volume variation $(S V V)$. $P P V, S P V$ and $S V V$ are defined by the relative difference in maximum and minimal pulse pressure $\left(P P_{\max }-P P_{\min }\right)$, systolic pressure $\left(S P_{\max }-S P_{\min }\right)$ and stroke volume $\left(S V_{\max }-S V_{\min }\right)$ over one respiratory cycle respec- 
tively [23],

$$
Q V(\%)=100 \cdot \frac{Q_{\max }-Q_{\min }}{\left(Q_{\max }+Q_{\min }\right) / 2},
$$

with Q= PP, SP, SV for PPV, SPV and SVV, respectively (see Figure 4). In the model, the dynamical indices are calculated breath-by-breath and subsequently averaged over the last four respiratory cycles.

Table 1: Basic hemodynamic variables and cardiac performance during steady state conditions. Normal values from humans [24] compared with the model variables during mechanical ventilation $\left(P E E P=5 \mathrm{cmH}_{2} \mathrm{O}, \mathrm{TV}=7 \mathrm{ml} \cdot \mathrm{kg}^{-1}, R R=12 \cdot \mathrm{min}^{-1}\right)$.

\begin{tabular}{|c|c|c|}
\hline \multicolumn{3}{|c|}{ Basic hemodynamic variables } \\
\hline Variable & Normal & Model \\
\hline Heart rate $\left(\mathrm{min}^{-1}\right)$ & $70-109$ & 75 \\
\hline Systemic systolic press $(\mathrm{mmHg})$ & $90-140$ & 109 \\
\hline Systemic diastolic press $(\mathrm{mmHg})$ & $60-90$ & 80 \\
\hline Central venous press $(\mathrm{mmHg})$ & $2-6$ & 4.3 \\
\hline Pulmonary systolic press $(\mathrm{mmHg})$ & $15-25$ & 27 \\
\hline Pulmonary diastolic press $(\mathrm{mmHg})$ & $8-15$ & 13 \\
\hline Pulmonary capillary press $(\mathrm{mmHg})$ & $6-12$ & 7.5 \\
\hline Pulmonary venous press $(\mathrm{mmHg})$ & $6-12$ & 6 \\
\hline Stroke volume index $\left(m l \cdot m^{-2}\right)$ & $40-70$ & 42 \\
\hline \multicolumn{3}{|c|}{ Cardiac performance } \\
\hline RVEDV $(m l)$ & $100-160$ & 145 \\
\hline Ejection fraction right ventricle $(\%)$ & $40-60$ & 56 \\
\hline
\end{tabular}

\subsection{Simulation results}

In order to validate the model, the simulation results are compared with data obtained from ventilated ICU patients, both from the literature and from data obtained in our ICU. First, basic hemodynamics are assessed during steady state conditions under mechanical ventilation and compared with reference values. Secondly, simulations were carried oud at two different levels of volume status and three different ventilatory settings and compared with data from literature. This second part focusses on the heart lung interaction with respect to the dynamic indices. To illustrate how the model can be used in clinical practice to help guide fluid therapy, we used the model to simulate fluid responsiveness in two patients and compared the results with the development of two patients undergoing fluid therapy on our ICU. These two patients 
were monitored as part of a clinical study regarding fluid responsiveness which was approved by the institutional review board of our institution.

Table 2: Comparison of the development of dynamic indices from the simulation-model with human (in vivo) data published in literature as a result of varying tidal volumes and respiratory rates $(R R)[25](N A=$ not available $)$.

\begin{tabular}{|c|c|c|c|c|c|c|}
\hline \multicolumn{7}{|c|}{ Dynamic indices } \\
\hline Variable & \multicolumn{6}{|c|}{ Before volume loading } \\
\hline & vivo & $\operatorname{sim}$ & vivo & $\operatorname{sim}$ & vivo & $\operatorname{sim}$ \\
\hline $\mathrm{TV}\left(\mathrm{ml} \cdot \mathrm{kg}^{-1}\right)$ & 5 & 5 & 10 & 10 & 15 & 15 \\
\hline $\operatorname{RR}\left(\min ^{-1}\right)$ & $18 \pm 2$ & 18 & $9 \pm 2$ & 9 & $6 \pm 1$ & 6 \\
\hline MAP $(m m H g)$ & $81 \pm 2$ & 83 & $82 \pm 2$ & 83 & $81 \pm 3$ & 83 \\
\hline $\mathrm{CVP}(\mathrm{mmHg})$ & $10 \pm 1$ & 3 & $10 \pm 1$ & 3 & $10 \pm 1$ & 4 \\
\hline $\operatorname{SVI}\left(m l \cdot m^{-2}\right)$ & $37 \pm 3$ & 33 & $35 \pm 3$ & 32 & $35 \pm 3$ & 31 \\
\hline PPV (\%) & $9.2 \pm 1$ & 6.7 & $14 \pm 2$ & 14.0 & $19 \pm 2$ & 20.8 \\
\hline SPV (\%) & NA & 5.5 & NA & 8.9 & NA & 10.7 \\
\hline \multirow[t]{3}{*}{ SVV (\%) } & $7 \pm 0.7$ & 7.4 & $15 \pm 2$ & 15.4 & $21 \pm 2.5$ & 23.8 \\
\hline & \multicolumn{6}{|c|}{ After volume loading } \\
\hline & vivo & $\operatorname{sim}$ & vivo & $\operatorname{sim}$ & vivo & $\operatorname{sim}$ \\
\hline $\mathrm{TV}\left(m l \cdot k g^{-1}\right)$ & 5 & 5 & 10 & 10 & 15 & 15 \\
\hline $\mathrm{RR}\left(\min ^{-1}\right)$ & $18 \pm 2$ & 18 & $9 \pm 2$ & 9 & $6 \pm 1$ & 6 \\
\hline MAP $(m m H g)$ & $88 \pm 3$ & 88 & $91 \pm 3$ & 88 & $92 \pm 3$ & 88 \\
\hline $\mathrm{CVP}(m m H g)$ & $12 \pm 1$ & 4 & $12 \pm 1$ & 5 & $12 \pm 1$ & 5 \\
\hline $\operatorname{SVI}\left(m l \cdot m^{-2}\right)$ & $40 \pm 3$ & 42 & $40 \pm 3$ & 42 & $39 \pm 3$ & 41 \\
\hline PPV $(\%)$ & $6.3 \pm 1$ & 6 & $8.6 \pm 1$ & 9.2 & $15 \pm 2$ & 15.9 \\
\hline $\operatorname{SPV}(\%)$ & NA & 5.2 & NA & 7.0 & NA & 9.3 \\
\hline SVV (\%) & $5.3 \pm 1$ & 6.5 & $10 \pm 1$ & 9.9 & $16 \pm 2$ & 17.5 \\
\hline
\end{tabular}

\subsubsection{Basic hemodynamics}

Results of the simulation of basic hemodynamic variables during mechanical ventilation are shown in Table 1. All values are within the physiological range [24], except for the pulmonary systolic pressure, which was a bit higher, but this is not considered a clinically relevant difference. The cardiac volumes, as illustrated in Figure 5 are also within targeted values, together with the ejection fractions. 


\subsubsection{Dynamic indices}

Target values for the dynamic indices, as a result of the interaction between the ventilation and circulation are derived from a clinical study [25], where the influence of the depth of tidal volume (TV) on dynamic indices, both during the state of fluid responsiveness and after fluid loading, was systematically investigated. They found that in addition to intravascular volume status, dynamic indices were significantly affected by the depth of TV under mechanical ventilation when patients are ventilated with 5 , 10 and $15 \mathrm{ml} \cdot \mathrm{kg}^{-1}$. Hemodynamic and ventilation data before and after fluid loading of 20 patients, including 32 fluid challenges, are presented in Table 2 (in vivo data). Both before and after volume loading, dynamic indices at TV of 5 and $15 \mathrm{ml} \cdot \mathrm{kg}^{-1}$ differed significantly from those at $10 \mathrm{ml} \cdot \mathrm{kg}^{-1}$. As a result of volume loading, dynamic indices at the respective TV were significantly lower than the values prior to the volume loading of $6 \mathrm{ml} \cdot \mathrm{kg}^{-1}$. All data from the model (Table 2, model data), except for the central venous pressure (CVP), is comparable with the mean \pm standard deviation of the clinical data. The CVP however, is within the normal values as presented in Table 1.

\subsubsection{Prediction of volume responsiveness}

As an example of how the model can be used in mechanically ventilated patients in the ICU, to distinquish volume responders (change in $\mathrm{CO}>12 \%$ ) from non-responders, the characteristics from two patients admitted on our ICU are displayed in Table 3. Both patients were diagnosed as hypovolemic patients by the attending physician because of low blood pressure and urine output, and were therefore given intravascular volume to improve hemodynamics. Notice that both patients have a PPV higher than $12 \%$, which is the clinical treshold for fluid responsiveness [26]. However, the non-responder in Table 3 is ventilated with a relatively high tidal volume and low respiratory rate, which causes the patient's PPV to rise without actually being hypovolemic. As a result, the SVI of the patient on the left (responder) rises due the fluid infusion, in contast to the SVI of the non-responder. To use the model to simulate this response, first of all, patient characteristics like weight and length should be set correctly into the model. Furthermore, the right cardiovascular parameters and variables of the patient must be entered in combination with the ventilator settings. The cardiovascular variables include e.g. the heart rate, central venous pressure, mean arterial pressure and the value of the dynamic indices. Required ventilator settings include tidal volume, respiratory rate and airway pressure (to derive respiratory compliance). Simulation results are also presented in Table 3, showing a predicted response in SVI of 20 and $7 \%$ for the volume responder and non-responder, respectively, and would thereby have predicted volume responsiveness correctly. 


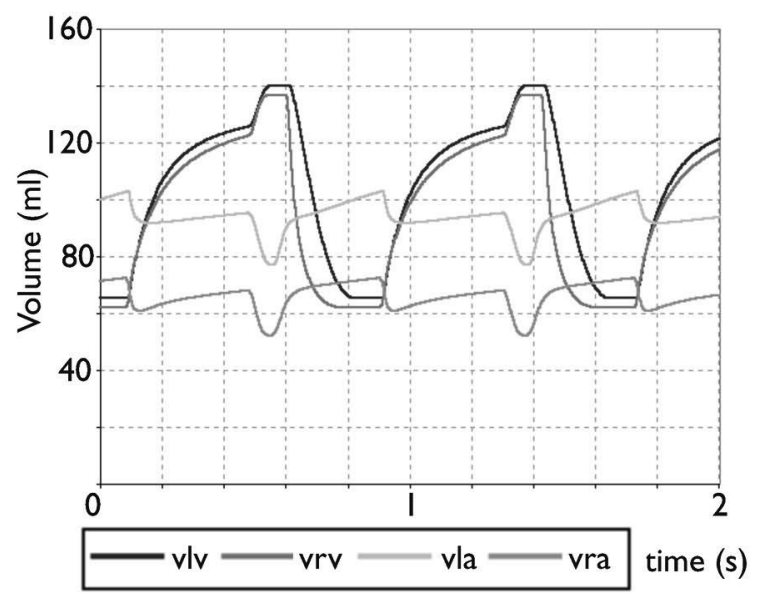

Figure 5: Volumes of the left and right ventricle (vlv, vrv, respectively) and the left and right atria (vla, vra, respectively) during two cardiac cycles.

Table 3: Example of how the model can be used in the ICU to distinquish volume responders from volume non-responders. Despite the fact that both patients have a pulse pressure variation above the clinical treshold of $12 \%$, one behaves like a responder and one as a nonresponder. This is partly due to the influences of the ventilatory setting (e.g. respiratory rate and tidal volume) on the dynamic indices.

\begin{tabular}{||l||l|l||}
\hline \multicolumn{2}{||c||}{ Prediction of volume responsiveness } \\
\hline Variable & \multicolumn{2}{||}{ Baseline } \\
\hline & Responder & Non-responder \\
\hline Tidal volume $\left(\mathrm{ml}^{\prime} \cdot \mathrm{kg}^{-1}\right)$ & 6.0 & 9.6 \\
Resp. rate $\left(\mathrm{min}^{-1}\right)$ & 14 & 10 \\
MAP $\left(\mathrm{mmHg}^{-1}\right)$ & 67 & 68 \\
HR $\left(\mathrm{min}^{-1}\right)$ & 102 & 93 \\
CVP $(\mathrm{mmH})$ & 6 & 7 \\
PPV $(\%)$ & 13.2 & 12.1 \\
SPV $(\%)$ & 8.0 & 7.39 \\
SVV $(\%)$ & 7.95 & 9.94 \\
\hline \multicolumn{1}{|c||}{} & \multicolumn{2}{c||}{ Cardiac output } \\
\hline SVI before fluid $\left(\mathrm{ml} \cdot \mathrm{m}^{-2}\right)$ & 27 & 30 \\
Measured change in SVI $(\%)$ & 13 & 6 \\
Simulated change in SVI $(\%)$ & 20 & 7 \\
\hline
\end{tabular}




\subsection{Discussion}

Reliable prediction of volume responsiveness in ICU patients is essential in daily clinical practice since hypovolemia may result in inadequate organ perfusion while inappropriate fluid administration can lead to organ disfunction and contributes to increased mortality [27]. Because of the lack of understanding of the underlying physiology of heart lung interaction, dynamic indices are difficult to interpret. This makes the dynamic indices only applicable in a selected population of patients. The proposed model is the first step towards a bedside clinical decision support system, and can be used as an educational tool as well.

In the present study, we developed a physiologically based mathematical model of the interaction between the respiratory and cardiovascular systems incorporating dynamic indices and fluid responsiveness. The model is able to simulate the highly interrelated processes of the relevant physiology and acts in a realistic way compared to clinical data regarding variations in volume status and tidal volume. Dynamic indices calculated by the model are comparable with those clinically measured in patients during two different levels of intravascular volume and three different ventilatory settings. By using models in combination with bedside monitoring of the cardiovascular and respiratory system, which is already done in patients receiving mechanical ventilation, models can be used for individual patients. While there are various model-based clinical decision support systems that use patient specific characteristics [28-30], to our knowledge this is the first model aimed at the decisions regarding volume management at the ICU.

Although we illustrated that the presented model is able to discriminate a responder from a nonresponder despite the fact that both have comparable values of dynamic indices, more effort should be made to make the model patient-specific before it can be used as a bedside decision support system. This can be done, for example, by incorporating pulse contour analysis. Thereby, the patient's peripheral vascular resistance can be calculated and from inspiratory volumes and pressures the respiratory compliances could be derived which also would make it possible to simulate individual patients more realistically. Implementation of these patient-specific physiological characteristics will make it possible to simulate individual patients more realistically and will improve the model's reliability concerning the prediction of volume responsiveness.

Besides serving as a decision support system, the model can also be used for educational purposes. Connecting the model to a simulator that incorporates a user interface for adjusting variables and parameters and an output monitor to visualize model output, will allow the different effects of respiratory parameters or patient specific physiology on the dynamic indices to be discriminated and thereby gaining insight into their contribution on the dynamic indices. This ability makes the simulator suitable as a supportive training tool for the understanding of the influences of the 
patient specific physiology and ventilatory parameter on, and thereby the interpretation of, dynamical indices regarding fluid responsiveness when accompanied by an educational program.

We also wish to address several limitations of our study. First, in humans, the exact contribution of each of the five mechanisms in the heart-lung interaction is not known. In the current model, their contribution is a weighted estimation based on clinical observations. Here, additional clinical data is needed to quantify the relevance of the various mechanisms in more detail. Second, we assumed the arterial and venous compliances to be constant. This, of course, is a simplification of reality because the volume-pressure relationship of a vessel is not linear. In general, the compliance decreases at higher pressures and volumes. On the other hand, in comparison with venous compliance, arterial compliance is reasonably constant, so introducing a constant arterial compliance will not introduce a large error. In addition, venous compliance at low pressures is also near to linear. Because of the hypovolemic conditions in which the simulated patients will mostly be situated in, and because of the fact that an increase in intrathoracic pressure will decrease transmural venous pressure, low transmural pressures are likely to exist. Furthermore, the vascular smooth muscle contraction influences vascular compliance. This contraction of the smooth muscles in the venous compartment, which is particularly important for the regulation of venous pressure and cardiac preload, is modeled by adjusting the unstressed volume of the venues by the baroreflex. In this way, smooth muscle contraction also influenced the pressure-volume relationship. Therefore, we think it is appropriate to assume a constant compliance for the various vessels. Third, a more detailed lung model could be implemented in the future for a more realistic simulation of airway pressures. In the current model, only three compartments describe the respiratory system. Finally, recent research shows that increased intra-abdominal pressure also plays a role in the heart-lung interaction [31]. Since the current model does not include an intra-abdominal cavity, this influence can not be taken into account. To do so, an intra-abdominal cavity should be implemented together with intra-abdominal arteries and veins that are influenced by this surrounding pressure.

\subsection{Conclusion}

The presented model is an improved version of original cardiovascular models, which describe the non-linear cardiac elastances, and extended with a respiratory system and several mechanisms describing the interaction between both. It is able to realistically simulate the relation between patient characteristics, ventilatory settings, volume status and dynamic indices which are relevant in predicting volume responsiveness. The model is able to discriminate volume responders from nonresponders but has to be developed further to make it generally applicable. The model can also be used as 
a didactical tool, allowing physiologists and clinicians to understand these complex relationships.

\section{References}

[1] F. Michard and J. L. Teboul, "Predicting fluid responsiveness in icu patients: a critical analysis of the evidence," Chest, vol. 121, no. 6, pp. 2000-8, 2002.

[2] A. Kramer, D. Zygun, H. Hawes, P. Easton, and A. Ferland, "Pulse pressure variation predicts fluid responsiveness following coronary artery bypass surgery," Chest, vol. 126, no. 5, pp. 1563-8, 2004.

[3] F. Michard, "Changes in arterial pressure during mechanical ventilation," Anesthesiology, vol. 103, no. 2, pp. 419-28, 2005.

[4] S. Magder, "Clinical usefulness of respiratory variations in arterial pressure," Am J Respir Crit Care Med, vol. 169, no. 2, pp. 151-5, 2004.

[5] P. E. Marik, R. Cavallazzi, T. Vasu, and A. Hirani, "Dynamic changes in arterial waveform derived variables and fluid responsiveness in mechanically ventilated patients: a systematic review of the literature," Crit Care Med, vol. 37, no. 9, pp. 2642-7, 2009.

[6] B. Lansdorp, J. Lemson, M. J. van Putten, A. de Keijzer, J. G. van der Hoeven, and P. Pickkers, "Dynamic indices do not predict volume responsiveness in routine clinical practice," British journal of anaesthesia, vol. 108, no. 3, pp. 395-401, 2012.

[7] V. C. Rideout, "Cardiovascular system simulation in biomedical engineering education," IEEE Trans Biomed Eng, vol. 19, no. 2, pp. 101-7, 1972.

[8] W. L. van Meurs, E. Nikkelen, and M. L. Good, "Pharmacokineticpharmacodynamic model for educational simulations," IEEE Trans Biomed Eng, vol. 45, no. 5, pp. 582-90, 1998.

[9] J. H. van Oostrom, S. Kentgens, J. E. Beneken, and J. S. Gravenstein, "An integrated coronary circulation teaching model," J Clin Monit Comput, vol. 20, no. 4, pp. 235-42, 2006.

[10] H. A. Rossow and J. H. Stern, "Teaching comparative metabolism using a graphic computer model, virtual tissue," Advances in Physiology Education, vol. 35, no. 1, pp. 99-102, 2011. 
[11] C. Luo, D. L. Ware, J. B. Zwischenberger, and J. Clark, J. W., "Using a human cardiopulmonary model to study and predict normal and diseased ventricular mechanics, septal interaction, and atrio-ventricular blood flow patterns," Cardiovascular engineering, vol. 7, no. 1, pp. 17-31, 2007.

[12] R. Warwick, M. Pullan, and M. Poullis, "Mathematical modelling to identify patients who should not undergo left ventricle remodelling surgery," Interact Cardiovasc Thorac Surg, 2010.

[13] V. K. Sud, R. Srinivasan, J. B. Charles, and M. W. Bungo, "Effects of lowerbody negative pressure on blood flow with applications to the human cardiovascular system," Medical and biological engineering and computing, vol. 31, no. 6, pp. 569-75, 1993.

[14] J. Messerges, "Modeling systolic pressure variation due to positive pressure ventilation," Conf Proc IEEE Eng Med Biol Soc, vol. 1, pp. 1806-9, 2006.

[15] T. Heldt, E. B. Shim, R. D. Kamm, and R. G. Mark, "Computational modeling of cardiovascular response to orthostatic stress," Journal of applied physiology, vol. 92, no. 3, pp. 1239-54, 2002.

[16] J. E. W. Beneken, A mathematical approach to cardio-vascular function; the uncontrolled human system, ser. Institute of Medical Physics. [Internal] report no. 2-4-5/6. Utrecht: Plenum Press, 1965.

[17] J. E. Beneken and V. C. Rideout, "The use of multiple models in cardiovascular system studies: transport and perturbation methods," IEEE Trans Biomed Eng, vol. 15 , no. 4, pp. 281-9, 1968.

[18] J. A. Goodwin, W. L. van Meurs, C. D. Sa Couto, J. E. Beneken, and S. A. Graves, "A model for educational simulation of infant cardiovascular physiology," Anesth Analg, vol. 99, no. 6, pp. 1655-64, table of contents, 2004.

[19] W. L. v. Meurs, Modeling and simulation in biomedical engineering : applications in cardiorespiratory physiology. New York: McGraw-Hill Professional ; London : McGraw-Hill [distributor], 2011.

[20] A. C. Guyton, Textbook of medical physiology, 7th ed. Philadelphia: Saunders, 1986.

[21] A. M. Gordon, A. F. Huxley, and F. J. Julian, "The variation in isometric tension with sarcomere length in vertebrate muscle fibres," J Physiol, vol. 184, no. 1, pp. 170-92, 1966. 
[22] S. C. Niranjan, A. Bidani, F. Ghorbel, J. B. Zwischenberger, and J. Clark, J. W., "Theoretical study of inspiratory flow waveforms during mechanical ventilation on pulmonary blood flow and gas exchange," Comput Biomed Res, vol. 32, no. 4, pp. 355-90, 1999.

[23] F. Michard, D. Chemla, C. Richard, M. Wysocki, M. R. Pinsky, Y. Lecarpentier, and J. L. Teboul, "Clinical use of respiratory changes in arterial pulse pressure to monitor the hemodynamic effects of peep," Am J Respir Crit Care Med, vol. 159, no. 3, pp. 935-9, 1999.

[24] P. Marino, The ICU book, 3rd ed. Baltimore: Lippincott Williams \& Wilkins, 2006.

[25] D. A. Reuter, J. Bayerlein, M. S. Goepfert, F. C. Weis, E. Kilger, P. Lamm, and A. E. Goetz, "Influence of tidal volume on left ventricular stroke volume variation measured by pulse contour analysis in mechanically ventilated patients," Intensive Care Med, vol. 29, no. 3, pp. 476-80, 2003.

[26] F. Michard, S. Boussat, D. Chemla, N. Anguel, A. Mercat, Y. Lecarpentier, C. Richard, M. R. Pinsky, and J. L. Teboul, "Relation between respiratory changes in arterial pulse pressure and fluid responsiveness in septic patients with acute circulatory failure," Am J Respir Crit Care Med, vol. 162, no. 1, pp. 134-8, 2000.

[27] D. Payen, A. C. de Pont, Y. Sakr, C. Spies, K. Reinhart, and J. L. Vincent, “A positive fluid balance is associated with a worse outcome in patients with acute renal failure," Crit Care, vol. 12, no. 3, p. R74, 2008.

[28] H. Tsuruta, T. Sato, M. Shirataka, and N. Ikeda, "Mathematical model of cardiovascular mechanics for diagnostic analysis and treatment of heart failure: Part 1. model description and theoretical analysis," Medical and biological engineering and computing, vol. 32, no. 1, pp. 3-11, 1994.

[29] M. L. Neal and J. B. Bassingthwaighte, "Subject-specific model estimation of cardiac output and blood volume during hemorrhage," Cardiovascular engineering, vol. 7, no. 3, pp. 97-120, 2007.

[30] J. Kabal and B. Lagerman, "Computer-aided clinical decision system: Differential diagnosis and treatment of essential hypertension by a novel noninvasive hemodynamic analyzer," Cardiovasc Eng, vol. 5, no. 2, p. 14, 2005.

[31] J. Renner, M. Gruenewald, R. Quaden, R. Hanss, P. Meybohm, M. Steinfath, J. Scholz, and B. Bein, "Influence of increased intra-abdominal pressure on fluid responsiveness predicted by pulse pressure variation and stroke volume variation in a porcine model," Crit Care Med, vol. 37, no. 2, pp. 650-8, 2009. 


\section{Appendix A}

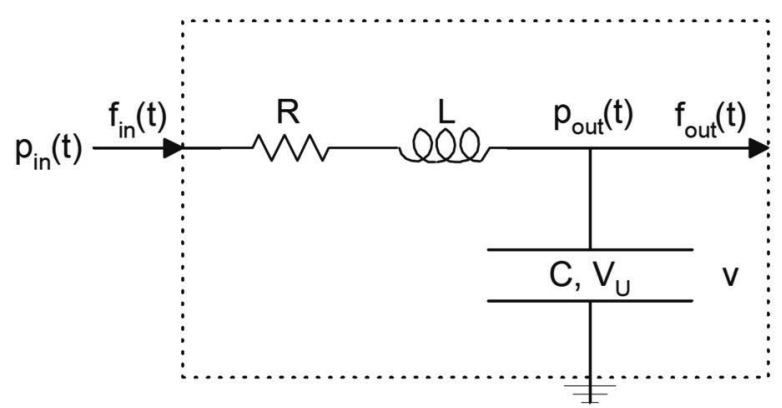

Figure A: The electrical equivalent of one compartment, with transmural pressure $(p, m m H g)$, blood flow $\left(f, m l \cdot \mathrm{sec}^{-1}\right)$, volume $(v, m l)$, resistance $\left(R, m m H g \cdot s e c \cdot m l^{-1}\right)$, Fluid inertance $\left(L, m m H g \cdot \sec ^{2} \cdot m l^{-1}\right)$, unstressed volume $\left(V_{u}, m l\right)$ and compliance $(C, m l$. $\left.m m \mathrm{Hg}^{-1}\right)$.

\section{Appendix B}


Table A: List of the used parameters and constants which are adjusted or can not directly be found in the refered literature. RA, RV, LA and LV are the right atrium, right ventricle, left atrium and left venricle, respectively.

\begin{tabular}{|c|c|c|c|}
\hline Symbol & & Description & Value \\
\hline \multicolumn{4}{|c|}{ cardiovascular system } \\
\hline RAORTA & & resistance aorta & $0.01 \mathrm{mmHg} \cdot \mathrm{ml}^{-1} \cdot \mathrm{s}$ \\
\hline EAORTA & & elastance aorta & $3.0 \mathrm{mmHg} \cdot \mathrm{ml}^{-1}$ \\
\hline vaorta & & unstressed volume aorta & $59 \mathrm{ml}$ \\
\hline RITHA & & resistance intrathoracic arteries & $0.029 \mathrm{mmHg} \cdot \mathrm{ml}^{-1} \cdot \mathrm{s}$ \\
\hline EITHA & & elastance intrathoracic arteries & $1.5 \mathrm{mmHg} \cdot \mathrm{ml}^{-1}$ \\
\hline vitha & & unstressed volume intrathoracic arteries & $172.0 \mathrm{ml}$ \\
\hline LITHA & & inertance intrathoracic arteries & $0.0015 \mathrm{mmHg} \cdot \mathrm{ml}^{-1} \cdot \mathrm{s}^{2}$ \\
\hline & $e_{\min }$ & minimum elastance & $0.1 \mathrm{mmHg} \cdot \mathrm{ml}^{-1}$ \\
\hline & $e_{\max }$ & maximum elastance & $0.2 \mathrm{mmHg} \cdot \mathrm{ml}^{-1}$ \\
\hline & $V_{u}$ & unstressed volume & $30.0 \mathrm{ml}$ \\
\hline \multirow[t]{3}{*}{$\mathrm{RV}$} & $e_{\min }$ & minimum elastance & $0.045 \mathrm{mmHg} \cdot \mathrm{ml}^{-1}$ \\
\hline & $e_{\max }$ & maximum elastance & $1.2 \mathrm{mmHg} \cdot \mathrm{ml}^{-1}$ \\
\hline & $V_{u}$ & unstressed volume & $40.0 \mathrm{ml}$ \\
\hline \multirow[t]{3}{*}{ LA } & $e_{\min }$ & minimum elastance & $0.12 \mathrm{mmHg} \cdot \mathrm{ml}^{-1}$ \\
\hline & $e_{\max }$ & maximum elastance & $0.2 \mathrm{mmHg} \cdot \mathrm{ml}^{-1}$ \\
\hline & $V_{u}$ & unstressed volume & $30.0 \mathrm{ml}$ \\
\hline \multirow[t]{3}{*}{ LV } & $e_{\min }$ & minimum elastance & $0.08 \mathrm{mmHg} \cdot \mathrm{ml}^{-1}$ \\
\hline & $e_{\max }$ & maximum elastance & $4.0 \mathrm{mmHg} \cdot \mathrm{ml}^{-1}$ \\
\hline & $V_{u}$ & unstressed volume & $30 \mathrm{ml}$ \\
\hline$e_{\text {diast }}^{\max }$ & & maximum increase in diastolic elastance & 0.5 \\
\hline$k$ & & constant & 4 \\
\hline$m$ & & constant & 8 \\
\hline \multicolumn{4}{|c|}{ baroreflex } \\
\hline$h r_{0}$ & & reference value heart rate & $73 \mathrm{~min}^{-1}$ \\
\hline$C_{h r / m a p}$ & & gain heart rate & \\
\hline svr $r_{0}$ & & reference value systemic vascular resistance & $0.83 \mathrm{mmHg} \cdot \mathrm{sec} \cdot \mathrm{ml}^{-1}$ \\
\hline$C_{\text {svr } / \text { map }}$ & & gain systemic vascular resistance & -0.25 \\
\hline contr $_{0}$ & & reference value contractility LV & $4.0 \mathrm{mmHg} \cdot \mathrm{ml}^{-1}$ \\
\hline contr $_{0}$ & & reference value contractility RV & $1.2 \mathrm{mmHg} \cdot \mathrm{ml}^{-1}$ \\
\hline$C_{\text {contr/mat }}$ & & gain contractility & -0.25 \\
\hline & & reference value unstressed venous volume & $2253 \mathrm{ml}$ \\
\hline$C_{V u / m a p}$ & & gain unstressed venous volume & 0.25 \\
\hline$M A P_{s p}$ & & setpoint value of map & $88 m m H G$ \\
\hline$B R_{\min }$ & & map with maximal baroreceptor activity & $68 m m H G$ \\
\hline$B R_{\max }$ & & map with minimal baroreceptor activity & $108 m m H G$ \\
\hline \multicolumn{4}{|c|}{ respiratory system } \\
\hline$C_{\text {lung }}$ & & lung compliance & $0.11 \mathrm{ml} \cdot \mathrm{mmHg}^{-1}$ \\
\hline$C_{\text {wall }}$ & & chest wall compliance & $0.19 \mathrm{ml} \cdot \mathrm{mmHg}^{-1}$ \\
\hline$R_{a w}$ & & airway resistance & $2.2 \mathrm{mmHg} \cdot \mathrm{sec} \cdot \mathrm{ml}^{-1}$ \\
\hline \multicolumn{4}{|c|}{ interaction } \\
\hline$R_{v c, 0}$ & & normal value resistance vena cava & $0.002 \mathrm{mmHg} \cdot \mathrm{sec} \cdot \mathrm{ml}^{-1}$ \\
\hline$C_{R, v c}$ & & constant & 0.021 \\
\hline$V_{u, p c, 0}$ & & normal value $V_{u}$ pulm capillaries & $60 \mathrm{ml}$ \\
\hline$R_{p c, 0}$ & & normal value pulm vasc resis & $0.095 \mathrm{mmHg} \cdot \mathrm{sec} \cdot \mathrm{ml}^{-1}$ \\
\hline$C_{V, p c}$ & & constant & 0.012 \\
\hline$C_{R, p c}$ & & constant & 0.09 \\
\hline
\end{tabular}




\section{Part III \\ Non-invasive techniques to assess fluid responsiveness}





\title{
9
}

\section{Validation of non-invasive pulse contour cardiac output using finger arterial pressure in cardiac surgery patients requiring fluid therapy}

\author{
C. Hofhuizen, B. Lansdorp, J.G. van der Hoeven, G.J. Scheffer, J. Lemson. \\ Journal of Critical Care, 01/2014 29(1): 161-165
}

\footnotetext{
Introduction. Nexfin ${ }^{\mathrm{TM}}$ allows for non-invasive continuous monitoring of blood pressure $\left(A B P_{N I}\right)$ and cardiac output $\left(C O_{N I}\right)$ by measuring finger arterial pressure (FAP). To evaluate the accuracy of FAP in measuring $A B P_{N I}$ and $C O_{N I}$ as well as the adequacy of detecting changes in $\mathrm{ABP}$ and $\mathrm{CO}$, we compared FAP to intra-arterially measured blood pressure $\left(A B P_{A I}\right)$ and transpulmonary thermodilution $\left(C O_{T D}\right)$ in post cardiac surgery patients during a fluid challenge (FC).

Methods. 20 sedated patients post-cardiac surgery were included and 28 FC's were performed. ABP and $\mathrm{CO}$ were simultaneously collected before and after a FC and we compared $\mathrm{CO}$ and blood pressure. Results. FAP was obtainable in all patients. When comparing $A B P_{N I}$ to $A B P_{I A}$, bias was $2.7 \mathrm{mmHg}$ (LOA 22.2), $4.9 \mathrm{mmHg}$ (LOA 13.6) and $4.2 \mathrm{mmHg}$ (LOA 13.7) for systolic, diastolic and mean arterial pressure, respectively. Concordance between changes in $A B P_{N I}$ and $A B P_{I A}$ was $100 \%$. Mean bias between $C O_{N I}$ and $C O_{T D}$ was -0.26 (LOA 2.2), with a percentage error of $38.9 \%$. Concordance between changes in $C O_{N I}$ vs $C O_{T D}$ and was $100 \%$.

Conclusion. Finger arterial pressure reliably measures $\mathrm{ABP}$ and adequately tracks changes in $\mathrm{ABP}$. $C O_{N I}$ is not interchangeable with $C O_{T D}$, However it follows changes in CO closely.
} 


\subsection{Introduction}

Maintaining adequate tissue perfusion and oxygenation is of paramount importance during anesthesia and in the critical care environment. Establishing an adequate cardiac output (CO) is an essential determinant of this therapeutic goal. Therefore, over the last decade, there has been an increasing interest in the continuous measurement of $\mathrm{CO}$ under various clinical conditions. Thermodilution is the clinical gold standard for $\mathrm{CO}$ measurement [1], but this technique requires the placement of a specific intra-arterial or pulmonary artery catheter that might lead to various complications. Therefore, there is a growing need for minimally invasive and continuous CO monitoring. This new technique should meet the desired requirements of accuracy, operator independence, safety, ease of application and continuous use [2]. Furthermore, a fast continuous method could be beneficial in tracking $\mathrm{CO}$ changes as a result of diagnostic maneuvers and interventions. Although a number of methods are available to measure $\mathrm{CO}$ non-invasively, none of these techniques answers to all requirements, and therefore the method of choice will depend on the physician 's experience, the patient and the clinical situation. The Nexfin device (Edwards Lifesciences, Irvine, CA, USA) measures blood pressure and $\mathrm{CO}$ continuously and non-invasively by measuring finger arterial pressure (FAP). The device uses a finger cuff to construct an arterial blood pressure waveform using a technique that is based on the volume clamp method developed by Peaz and the physiocal criteria of Wesseling [3, 4]. Nexfin CO-TREK is a mathematical model incorporated in the software that calculates beat-to-beat stroke volume (SV) using the arterial blood pressure waveform [5]. This combination enables the continuous measurement of blood pressure and $\mathrm{CO}$ in a non-invasive manner. With the application of the CO-TREK algorithm, it is also possible to determine the $\mathrm{CO}$ offline using the invasive blood pressure signal measured with an intra-arterial catheter. A small number of studies has been undertaken to assess the accuracy of FAP CO in determining absolute $\mathrm{CO}$ levels with varying results [6-8]. However, an important part of hemodynamic optimization is the effect of a given treatment such as fluid expansion. Therefore, we assessed the accuracy of FAP in tracking $\mathrm{CO}$ changes after a fluid challenge. In this study we compared CO-TREK CO derived from the non-invasive finger blood pressure signal $\left(\mathrm{CO}_{N I}\right)$ and CO-TREK CO derived from the intra-arterial blood pressure signal $\left(\mathrm{CO}_{I A}\right)$ with $\mathrm{CO}$ derived from transpulmonary thermodilution $\left(\mathrm{CO}_{T D}\right)$ using $\mathrm{PiCCO}^{\circledR}$ (Pulsion Medical Systems, Munich, Germany) as the reference method. We also compared FAP blood pressure $\left(A B P_{N I}\right)$ to intra-arterially measured blood pressure $\left(A B P_{I A}\right)$. 


\subsection{Methods}

\subsubsection{Subjects}

With the approval of the institutional review board and after obtaining participants written informed consent, 20 patients admitted to the intensive care unit (ICU) following elective conventional cardiac surgery were studied. Exclusion criteria were cardiac arrhythmias, preoperative inotropic or intra-aortic balloon pump support and patients requiring emergency or redo cardiac surgery.

\subsubsection{Haemodynamic monitoring}

Anesthesia was according to the institutional protocol at the discretion of the attending anaesthesiologist. A 20 GA right radial intra-arterial catheter (Becton Dickinson and Co., Franklin Lakes, NJ, USA) was introduced before anesthesia induction and connected via standard low compliant tubing to a disposable pressure transducer (Edwards Lifesciences, Irvine, CA, USA). After induction, a two-lumen central venous catheter was inserted in the right internal jugular vein for the measurement of central venous pressure (CVP). $\mathrm{CO}$ was monitored using the $\mathrm{PiCCO}{ }^{\circledR}$ monitor and a 5-F thermistor-tipped arterial $\mathrm{PiCCO}^{\circledR}$ catheter inserted in the femoral artery (Pulsion Medical Systems, Munich, Germany). All pressure monitors were zeroed at the midaxillary line. Signals were recorded simultaneously using a sample rate of $200 \mathrm{~Hz}$ and stored on a hard disk.

\subsubsection{FAP CO monitoring}

FAP is a device for non-invasive and continuous measurement of blood pressure using a finger cuff. A photoplethysmograph mounted inside the finger cuff detects changes in finger arterial diameter. Using a fast pneumatic system the diameter of the finger artery can be held at a constant level by rapidly varying the pressure in the finger cuff air bladder. This is called the volume clamp method. If the artery is clamped at the correct diameter, the pressure in the air bladder is identical to the pressure inside the artery, and finger arterial pressure is measured. The correct arterial diameter is determined at regular intervals during a blood pressure measurement, by a physiological calibration called physiocal [9]. Nexfin applies a waveform and level correction methodology to reconstruct finger arterial pressure to the arterial pressure waveform at brachial artery level [10]. FAP calculates beat-to-beat SV by dividing the area under the systolic portion of the arterial pressure curve by the aortic input impedance [11]. The value of this aortic input impedance is determined from a three-element Windkessel model described by Westerhof et. al. [12]. In this model the non-linear effect of mean pressure as well as the influence of the patients age, height, weight 
and sex on aortic mechanical properties are incorporated. The algorithm that converts finger arterial waveform to $\mathrm{CO}$ is called CO-TREK. The non-invasive arterial signal $\left(A B P_{N I}\right)$ and $\mathrm{CO}\left(C O_{N I}\right)$ were obtained using an appropriate size finger cuff applied to the midphalanx of the left middle or index finger according to guidelines provided by the manufacturer. The finger with the cuff was positioned at the midaxillary line and the finger was checked regularly for signs of tissue hypoxia. To determine whether inaccuracies in the predictive value of the non-invasive finger signal were caused by the non-invasive character of the measurement or were caused by inaccuracies in the CO-TREK algorithm itself, we also calculated $\mathrm{CO}$ using the stored intra-arterial blood pressure signal $\left(\mathrm{CO}_{I A}\right) . C O_{I A}$ was calculated offline using the CO-TREK algorithm and we compared this to $C O_{T D}$.

\subsubsection{Design}

After surgery, patients were admitted to the ICU and sedated with midazolam. FAP recording started immediately after arrival at the ICU. FAP measurement was considered adequate if physiocal occurred at intervals longer than 30 seconds. The IAP measurement was checked for quality by visually inspecting the waveform and performing fast slush test. If, at the discretion of the attending intensive care physician, a fluid challenge (FC) was indicated, a transpulmonary thermodilution measurement was performed by three injections of $15 \mathrm{ml}$ of ice-cold saline through the central venous catheter before the FC (T1) and 5 minutes after completion of FC (T2). If a difference of more than $20 \%$ occurred between the three three thermodilution measurements, injection was repeated. Criterion for a FC was presence of a mean arterial pressure below $70 \mathrm{mmHg}$. To evaluate the clinical effect of the fluid challenge, another thermodilution measurement was performed 30 min after completion of the FC if the clinical situation permitted, e.g. patient still fully sedated, no change in inotropic medication, etc (T3). The FC was performed by infusing $6 \mathrm{ml}$ per $\mathrm{kg}$ ideal body weight of a 130/0.4 6\% HES solution (Fresenius Kabi, the Netherlands) over a period of 15 minutes. Simultaneous data of $C O_{T D}, C O_{N I}, A B P_{N I}$ and $A B P_{I A}$ were collected throughout the postoperative period in the ICU until the patient was extubated according to local standard operating procedures.

\subsubsection{Statistical analysis}

To compare $C O_{N I}$ and $C O_{I A}$ to $C O_{T D}$ we averaged a 20 s time interval of the noninvasive finger measurement and intra-arterial measurement and compared it to the simultaneously performed $C O_{T D}$. This interval was chosen to evade periods of physiocal, which occurs at regular intervals during a FAP measurement. , The same time intervals were used to compare $A B P_{N I}$ to $A B P_{I A}$. Hemodynamic parameters were reported as mean standard deviation (SD). The Kolmogorov-Smirnov test was used to 
test the normality of the distribution. To assess agreement (bias and limits of agreement) between the invasive and non-invasive derived parameters Bland Altman analysis was used which was corrected for repeated measurements in one subject [13]. The mean percentage error was assessed using the Critchley and Critchley method [14]. Also, we defined an agreement tolerability interval ratio using a tolerability interval of $4 \mathrm{~L} / \mathrm{min}$ (4- $8 \mathrm{~L} / \mathrm{min})$ [15]. To assess the hemodynamic change that occurred we compared a time point to the hemodynamic measurement taken earlier, i.e. T1 to T2 and $\mathrm{T} 2$ to T3. For assessing the trending ability of $\mathrm{CO}_{T D}$, we determined concordance using a four-quadrant plot. Changes in blood pressure and $\mathrm{CO}$ smaller than $5 \%$ were not considered clinically relevant and therefore excluded. Hereafter we constructed a polar plot of $\mathrm{CO}$ changes among consecutive time points described by Critchley et al $[16,17]$. Influence of temperature and use of vasoactive drugs on bias was checked using the student's t-test. Statistical analyses were carried out using GraphPad Prism version 5.01 (GraphPad software Inc., La Jolla, CA, USA). Assuming a SD of 1.33 $\mathrm{L} / \mathrm{min} \mathrm{L}$ (7) we needed a minimum of 69 simultaneous measurements to achieve a confidence interval of $0.25 \mathrm{~L} / \mathrm{min}$. Assuming we would perform 4 measurements in each subject we included 20 patients.

\subsection{Results}

20 patients were included in the study. In all patients a sufficient quality FAP waveform was obtained. There were no signs of tissue hypoxia distal to the finger cuff and no adverse events were noted. Twenty-eight fluid challenges were performed in 19 patients. One patient did not receive a FC. Due to technical difficulties intra-arterial blood pressure recording failed in 4 patients, which led to 66 pairs of $C O_{N I}$ and $C O_{T D}$ and 54 pairs of $\mathrm{ABP}$ and $C O_{N I}, C O_{I A}$ and $C O_{T D}$ data. Patient baseline characteristics and hemodynamic data are shown in Table 1 and 2 respectively.

\subsection{1 $A B P_{N I}$ vs $A B P_{I A}$}

Mean bias (SD) and limits of agreement (LOA) between $A B P_{N I}$ and $A B P_{I A}$ was 2.7 $\mathrm{mmHg}(\mathrm{LOA} \pm 22.2), 4.9 \mathrm{mmHg}(\mathrm{LOA} \pm 13.6)$ and $4.2 \mathrm{mmHg}(\mathrm{LOA} \pm 13.7)$ for systolic, diastolic and mean arterial pressure, respectively. Comparison between $A B P_{N I}$ and $A B P_{I A}$ for mean arterial pressure by a Bland-Altman analysis is depicted in Figure 1. $A B P_{N I}$ tracked changes of $A B P_{I A}$ with a mean bias of 0 (LOA \pm 13.7 ), $-1(\mathrm{LOA} \pm 10.0)$ and $-1(\mathrm{LOA} \pm 9.6) \mathrm{mmHg}$ for systolic, diastolic and mean arterial pressure respectively. Concordance between $A B P_{N I}$ and $A B P_{I A}$ was $100 \%$ using the $5 \%$ exclusion zone. Figure 2 shows a polar plot depicting the changes in mean arterial pressure for the two methods. Mean polar angle was 10.4 degrees with a standard deviation of 10.3 degrees. All data points lie between the 30 degrees radial limits. 


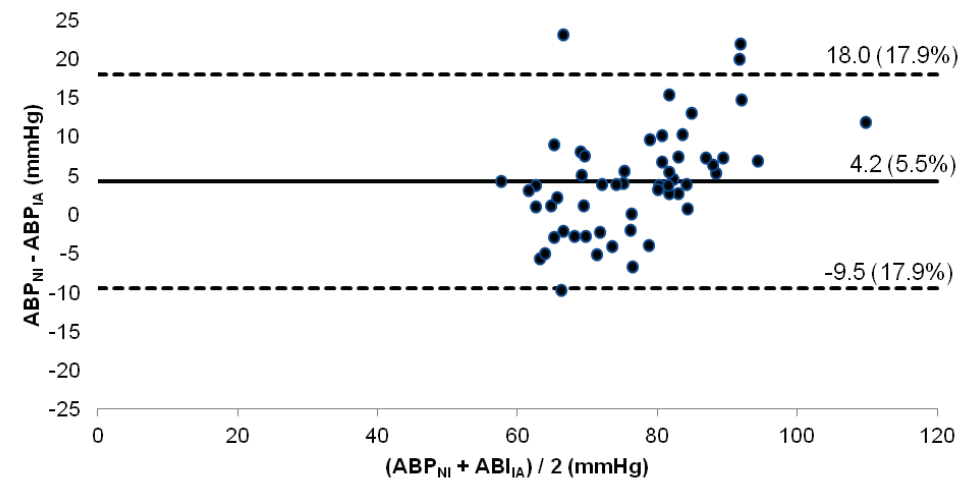

Figure 1: Agreement between $A B P_{I A}$ and $A B P_{N I}$ depicted in a Bland-Altman plot for mean arterial pressure. $A B P_{I A}=$ Arterial pressure measured with an intra-arterial cannula, $A B P_{N I}$ $=$ Arterial pressure measured with FAP.

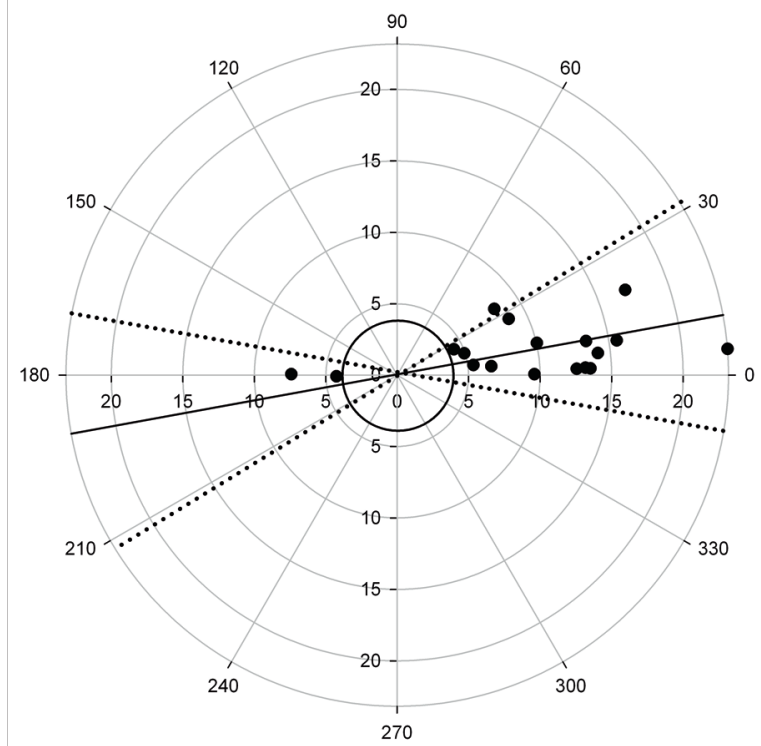

Figure 2: Polar plot comparing the changes between $A B P_{N I} v s A B P_{I A}$ for mean arterial pressure before and after a fluid challenge using a 5\% exclusion zone. The horizontal and vertical axes represent the mean change in blood pressure, agreement is represented by the angle with the horizontal axis. Solid line $=$ mean polar angle, dotted lines $=95 \%$ confidence interval. 
Table 1: Patient characteristics and baseline parameters, $C A B G=$ coronary artery bypass grafting, $A V R=$ aortic valve replacement, $F C=$ fluid challenge, $I B W=$ ideal body weight . Data presented as mean(range) or mean $\pm S D$

\begin{tabular}{|c|c|}
\hline \multicolumn{2}{|l|}{ patient characteristics \& baseline parameters } \\
\hline Patient (\#) & 19 \\
\hline Male/Female (\#) & $17 / 2$ \\
\hline Age $(y r)$ & $67(50-81)$ \\
\hline $\operatorname{BMI}\left(k g / m^{2}\right)$ & $28.3(21.4-37.1)$ \\
\hline Type of surgery (\#) & \\
\hline $\mathrm{CABG}$ & 15 \\
\hline AVR & 2 \\
\hline $\mathrm{CABG}+\mathrm{AVR}$ & 2 \\
\hline APACHEII & $12.5(5-20)$ \\
\hline History (\#m) & \\
\hline Ischaemic heart disease & $18(90 \%)$ \\
\hline Hypertension & $14(70 \%)$ \\
\hline Diabetes mellitus & $7(35 \%)$ \\
\hline Obesity & $6(30 \%)$ \\
\hline Core temperatur $\left(^{c}\right)$ at time of FC & $37.5(35.4-38.0)$ \\
\hline Tidal volume $\left(\mathrm{ml} \cdot \mathrm{kg}_{1} I B W\right)$ & $7.0 \pm 0.8$ \\
\hline Respiratory rate $(\mathrm{bpm})$ & $12.6 \pm 1.2$ \\
\hline Positive end-experitory pressure $\left(\mathrm{cmH}_{2} \mathrm{O}\right)$ & $6.2 \pm 1.8$ \\
\hline Plateau pressure $\left(\mathrm{CmH}_{2} \mathrm{O}\right)$ & $19.0 \pm 4.4$ \\
\hline Patients receiving norepinephrine (\#) & $8(40 \%)$ \\
\hline Mean dose $(\mathrm{mcg} / \mathrm{kg} / \mathrm{min})$ & $0.03(0.01-0.08)$ \\
\hline Patients receiving inotropic support (Dobutamine/Milrinone) (\#) & $4(20 \%)$ \\
\hline Mean dose $(\mathrm{mcg} / \mathrm{kg} / \mathrm{min})$ & $3.8(2.6-5.0)$ \\
\hline
\end{tabular}

\subsection{2 $C O_{N I}$ and $C O_{I A}$ vs $C O_{T D}$}

No significant difference was found between mean absolute values of $C O_{N I}$ and $C O_{T D}$. The relation between $C O_{N I}$ and $C O_{T D}$ is depicted in Figure 3. Mean bias between $C O_{N I}$ vs $C O_{T D}$ and $C O_{I A}$ vs $C O_{T D}$ was $-0.26(\mathrm{LOA} \pm 2.2) \mathrm{L} / \mathrm{min}$ and -0.78 $(\mathrm{LOA} \pm 1.9) \mathrm{L} / \mathrm{min}$, respectively. The percentage error between $C O_{N I}$ and $C O_{T D}$ was $38.9 \%$, the percentage error between $C O_{I A}$ and $C O_{T D}$ was $35.1 \%$. Agreement interval was $4.4 \mathrm{~L} / \mathrm{min}$ which did not meet the criterion of an agreement tolerability interval ratio of $4 \mathrm{~L} / \mathrm{min}$, representing marginal agreement. Bias was not influenced by temperature of the hand or the use of vasoactive medication. 
Table 2: Hemodynamic changes before and after a fluid challenge. Data are expresses as mean and standard deviation, $n=28,5$ pairs missing $C O_{I A}$ and $M A P_{I A}$ due to technical difficulties. ${ }^{*}$ significantly different from reference techniques $(p>0,05) . S A P=$ systolic arterial pressure, $D A P=$ diastolic arterial pressure, $M A P=$ mean arterial pressure, $C O=$ cardiac output

\begin{tabular}{||l|l|l|l||}
\hline & $\begin{array}{l}\text { Before fluid challenge } \\
\mathrm{T} 1(\mathrm{n}=28)\end{array}$ & $\begin{array}{l}5 \text { min after fluid } \\
\text { challenge } \\
\mathrm{T} 2(\mathrm{n}=28)\end{array}$ & $\begin{array}{l}30 \text { min after fluid } \\
\text { challenge } \\
\mathrm{T} 3(\mathrm{n}=10)\end{array}$ \\
\hline $\mathrm{SAP}_{N I}$ & $102.2 \pm 12.6$ & $113.3 \pm 16.7$ & $125.4 \pm 19.2$ \\
$\mathrm{SAP}_{I A}$ & $101.5 \pm 11.5$ & $116.7 \pm 17.1$ & $123.2 \pm 19.4$ \\
$\mathrm{DAP}_{N I}$ & $59.2 \pm 8.9$ & $63.0 \pm 9.7$ & $71.0 \pm 14.5$ \\
$\mathrm{DAP}_{I A}$ & $53.5 \pm 7.4$ & $58.2 \pm 5.9$ & $62.0 \pm 7.6$ \\
$\mathrm{MAP}_{N I}$ & $71.6 \pm 5.1$ & $81.8 \pm 12.0$ & $87.6 \pm 17.2$ \\
$\mathrm{MAP}_{I A}$ & $68.7 \pm 7.7$ & $77.4 \pm 8.3$ & $81.0 \pm 10.9$ \\
$\mathrm{CO}_{T D}$ & $5.31 \pm 1.26$ & $6.29 \pm 0.94$ & $5.81 \pm 1.36$ \\
$\mathrm{CO}_{N I}$ & $5.32 \pm 1.23$ & $6.12 \pm 1.03$ & $5.43 \pm 1.38$ \\
$\mathrm{CO}_{I A}$ & $4.91 \pm 0.88$ & $5.50 \pm 1.03$ & $5.66 \pm 0.99$ \\
\hline
\end{tabular}

\subsubsection{Changes in $C O_{N I}$ vs $C O_{T D}$}

The mean change in $\mathrm{CO}$ after a FC measured with thermodilution was 0.59 (range -1.0 2.4) $\mathrm{L} / \mathrm{min}$. Concordance rate between $C O_{N I}$ and $C O_{T D}$ and concordance rate between $C O_{I A}$ and $C O_{T D}$ were $100 \%$ and $91.7 \%$ respectively using the $5 \%$ exclusion zone. $C O_{N I}$ and $C O_{I A}$ tracked changes in $C O_{T D}$ with a mean bias of $-0.31 \mathrm{~L} / \mathrm{min}$ $(\mathrm{LOA} \pm 1.0)$ and $-0.25 \mathrm{~L} / \mathrm{min}(\mathrm{LOA} \pm 1.2)$ respectively. Figure 4 shows a polar plot depicting the changes in $\mathrm{CO}$ for two methods. Mean polar angle was 17.8 degrees with a standard deviation of 13.0 degrees. $82 \%$ of all data points lie within the 30 degrees radial limits.

\subsection{Discussion}

The main conclusions from our study are that in a population of patients after cardiac surgery blood pressure was accurately measured by FAP and also FAP was capable of tracking blood pressure changes. However both $C O_{N I}$ and $C O_{I A}$ were not interchangeable with transpulmonary thermodilution for $\mathrm{CO}$ measurement although FAP shows strong tracking capabilities for $\mathrm{CO}$ changes after a FC. Bias and precision of diastolic and mean arterial pressure are within the limits set by the Association for the Advancement of Medical Instrumentation that allow for a maximum bias of $5 \mathrm{mmHg}$ and a maximal precision of $8 \mathrm{mmHg}$ [18]. These results are in concordance with 


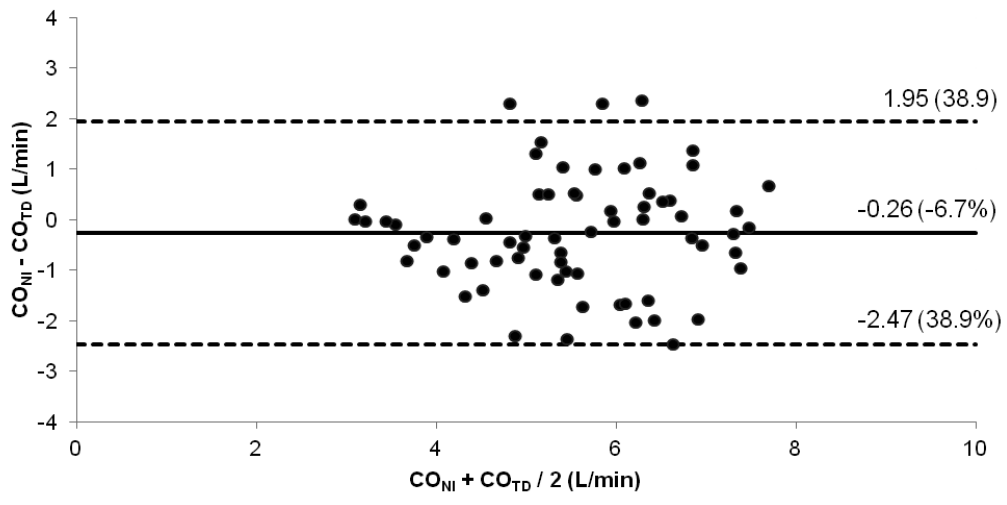

Figure 3: Agreement between $C O_{T D}$ and $C O_{N I}$ depicted in a Bland-Altman analysis. $C O_{T D}$ $=$ Cardiac output measured with PiCCO ${ }^{\circledR}, C O_{N I}=$ Cardiac output measured with FAP.

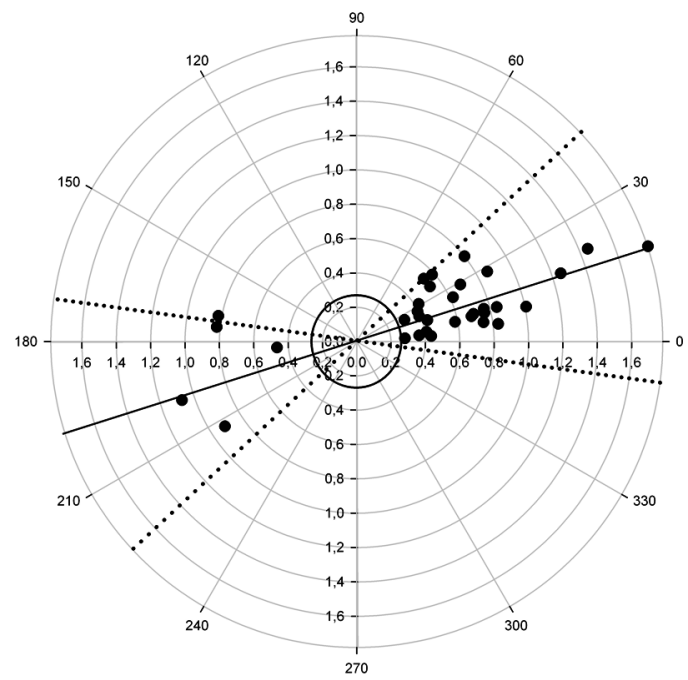

Figure 4: Polar plot comparing the changes between $C O_{N I}$ vs $C O_{T D}$ before and after a fluid challenge using a 5\% exclusion zone. The horizontal and vertical axes represent the mean change in cardiac output, agreement is represented by the angle with the horizontal axis. Solid line $=$ mean polar angle, dotted lines $=95 \%$ confidence interval. 
previous studies $[7,19,20]$ where FAP was plotted against intra-arterially measured blood pressure. Studies comparing $A B P_{N I}$ against a non-invasive blood pressure measurement technique (NIBD), such as oscillometry, show more diverse results, possibly reflecting the underlying variability of the NIBD [21, 22]. In this study, FAP was not interchangeable with transpulmonary thermodilution CO. Although bias was low, percentage error did not meet the criterion of a maximum percentage error of $30 \%$ proposed by Critchley and Critchley [16]. Accuracy of FAP was however, similar to the accuracy of $\mathrm{CO}_{I A}$, suggesting that inaccuracy of the CO-TREK algorithm is not caused by a less reliable non-invasive arterial signal. However, the technique of intra-arterially measured blood pressure relies on pressure differences where finger pressure measurement relies on arterial diameter changes. This fundamental difference may account for the difference between $C O_{N I}$ and $C O_{I A}$. Also, due to the nature of the measurement, FAP cannot be influenced by several factors that influence the accuracy of intra-arterially measured blood pressure such as kinking, presence of bubbles and blood clots or underdamping. Several other clinical studies evaluating the accuracy of FAP, show diverse results with percentage errors ranging from 25 to $50 \%[6-8,20,23,24]$. However, methodological differences such as reference techniques, use of vasoactive medication, patient characteristics and clinical situations ranging from critical care to outpatient evaluation preclude direct comparison and may account for this wide range in observed accuracy. In this study, $82 \%$ of all data points fall between the 30 degrees radial limits of agreement reflecting marginal trending. This finding is caused by the angular bias of 17.8 degrees. Although this angular bias is quite large, the spread around this angle was quite small with a SD of 13.0 degrees. This possibly reflects a significant offset in calibration where $C O_{T D}$ measured greater changes in $\mathrm{CO}$ than did $\mathrm{CO}_{N I}$. In this study, we obtained a good quality non-invasive signal in all patients despite the presence of vasoactive medication, hypothermia or the fact that most subjects had a history of hypertension and vascular disease. Although finger blood pressure and $\mathrm{CO}$ measurement is dependent of finger arterial flow, we found no correlation between bias and core temperature or the use of vasoactive medication [25]. However, norepinephrine dosage did not exceed $0,1 \mathrm{mcg} / \mathrm{kg} / \mathrm{min}$ and patients were not exposed to core temperatures below 35.4 Compared to a single $\mathrm{CO}$ measurement, tracking of $\mathrm{CO}$ changes to evaluate the clinical course or the effect of an instituted treatment may be a more valuable tool in patients with hemodynamic abnormalities. In this study, we found a high concordance between changes in $C O_{N I}$ and $C O_{T D}$, which corresponds with previous studies investigating the ability of FAP in tracking $\mathrm{CO}$ changes $[6,24,26]$. In one previous study also investigating the accuracy of FAP in tracking $\mathrm{CO}$ changes induced by fluid expansion, a polar angle of -1.2 was found. This difference might be explained by the larger exclusion zone of $15 \%$ that was used or the use of another reference technique, the pulmonary artery catheter. Several limitations of our study should be noted. 
First, we investigated a group of postoperative cardiac surgery patients under stable hemodynamic conditions. Although $45 \%$ of patients required inotropic or vasoactive medication, dosage was low. Our data are therefore not automatically transferable to other subgroups such as critically ill patients or trauma care. Also, the dosages of the inotropic and vasoactive medication were stable throughout the study, therefore acute changes in vascular tone were not investigated, Second, this study evaluates the accuracy of $\mathrm{CO}_{N I}$ in tracking $\mathrm{CO}$ changes after fluid expansion. However, $\mathrm{CO}$ changes after a FC were modest with a maximum of $2.03 \mathrm{~L} / \mathrm{min}$ in comparison with the hemodynamic response seen in septic patients. Further research in tracking $\mathrm{CO}$ changes in hemodynamic unstable patients is necessary. Third, this study is not designed to determine the accuracy of FAP in determining fluid responsiveness. In addition to $\mathrm{CO}$ measurement, the prediction of fluid responsiveness is an important tool to guide fluid resuscitation during hemodynamic optimization. Considering its desirable characteristics, FAP could provide a valuable monitor to determine fluid responsiveness. However, further research is mandatory to evaluate the accuracy and feasibility of FAP in the guidance of fluid optimization. In this study, we compared $\mathrm{CO}_{N I}$ to transpulmonary thermodilution. Although thermodilution is considered the gold standard for $\mathrm{CO}$ measurement, in previous studies transpulmonary thermodilution using $\mathrm{PiCCO}^{\circledR}$ has been shown to be less accurate during thoracic surgery or hemodynamic instability [27]. This may, in part, explain the wide percentage error found in the present study. Recently, Peyton et al. published a meta-analysis comparing 4 different minimally invasive $\mathrm{CO}$ monitors showing that none of the four methods tested achieved to meet the $30 \%$ limits of agreement with thermodilution [27]. They propose that a percentage error of $45 \%$ in agreement with thermodilution is a more realistic precision in clinical practice. $C O_{N I}$ does not meet the criterion of a maximum agreement tolerability interval ratio of $1 \mathrm{~L} / \mathrm{min}$ in the present study [15]. The concept set by Columb is interesting because it assesses the potential for misclassification of a measurement technique that could result in opposing interventions. It defines a priori what will constitute an acceptable agreement given a certain clinical situation. For example, misclassifying a patient with a low output state as a hyperdynamic patient can have severe clinical consequences and is therefore unacceptable. However, the concept does not define an acceptable agreement for a given measured hemodynamic value within a certain treatment population. For example, where a SD of $0.5 \mathrm{~L} / \mathrm{min}$ in a patient with a $\mathrm{CO}$ of $8 \mathrm{~L} / \mathrm{min}$ would be very satisfactory, the same $\mathrm{SD}$ in a patient with a mean $\mathrm{CO}$ of $3 \mathrm{~L} / \mathrm{min}$ could have severe consequences in clinical decision making. A concept that assesses agreement by considering clinical implications given a certain measurement value would be very interesting. In an editorial Feldman underlines that in evaluating the efficacy of a new $\mathrm{CO}$ monitor, one should consider a more dynamic approach incorporating not only bias and precision but also clinical utility and safety as well as impact on decision making. In addition, next 
to accuracy several desirable characteristics of a monitoring technique are defined, such as safety, operator independence, ease of use and continuous use [2]. Considering these characteristics, FAP could be a valuable monitor in the perioperative and critical care setting.

\subsection{Conclusion}

Finger arterial pressure is a promising technique for the determination of blood pressure and $\mathrm{CO}$. The technique is safe, fast, easy to use and provides a continuous noninvasive blood pressure and $\mathrm{CO}$ signal. It is reliable in the measurement of blood pressure and the tracking of blood pressure changes. Our data suggest that FAP CO is not interchangeable with transpulmonary thermodilution with a percentage error of $38.9 \%$. However, trending analysis showed a high concordance with thermodilution after a fluid challenge.

\section{References}

[1] J. Pugsley and A. B. Lerner, "Cardiac output monitoring: is there a gold standard and how do the newer technologies compare?" Seminars in cardiothoracic and vascular anesthesia, vol. 14, no. 4, pp. 274-82, 2010.

[2] J. N. Shephard, S. J. Brecker, and T. W. Evans, "Bedside assessment of myocardial performance in the critically ill," Intensive care medicine, vol. 20, no. 7, pp. 513-21, 1994.

[3] J. Penaz, "Criteria for set point estimation in the volume clamp method of blood pressure measurement," Physiological research / Academia Scientiarum Bohemoslovaca, vol. 41, no. 1, pp. 5-10, 1992.

[4] K. H. Wesseling, B. Dewit, G. M. A. Vanderhoeven, J. Vangoudoever, and J. J. Settels, "Physiocal, calibrating finger vascular physiology for finapres," Homeostasis in Health and Disease, vol. 36, no. 2-3, pp. 67-82, 1995.

[5] K. H. Wesseling, J. R. Jansen, J. J. Settels, and J. J. Schreuder, "Computation of aortic flow from pressure in humans using a nonlinear, three-element model," Journal of applied physiology, vol. 74, no. 5, pp. 2566-73, 1993.

[6] G. Chen, L. Meng, B. Alexander, N. P. Tran, Z. N. Kain, and M. Cannesson, "Comparison of noninvasive cardiac output measurements using the nexfin monitoring device and the esophageal doppler," Journal of clinical anesthesia, vol. 24, no. 4, pp. 275-83, 2012. 
[7] M. O. Fischer, R. Avram, I. Carjaliu, M. Massetti, J. L. Gerard, J. L. Hanouz, and J. L. Fellahi, "Non-invasive continuous arterial pressure and cardiac index monitoring with nexfin after cardiac surgery," British journal of anaesthesia, vol. 109, no. 4, pp. 514-21, 2012.

[8] L. W. Bogert, K. H. Wesseling, O. Schraa, E. J. Van Lieshout, B. A. de Mol, J. van Goudoever, B. E. Westerhof, and J. J. van Lieshout, "Pulse contour cardiac output derived from non-invasive arterial pressure in cardiovascular disease," Anaesthesia, vol. 65, no. 11, pp. 1119-25, 2010.

[9] K. H. Wesseling, "A century of noninvasive arterial-pressure measurement from marey to penaz and finapres," Homeostasis in Health and Disease, vol. 36, no. 2-3, pp. 50-65, 1995.

[10] P. Gizdulich, A. Prentza, and K. H. Wesseling, "Models of brachial to finger pulse wave distortion and pressure decrement," Cardiovascular research, vol. 33, no. 3, pp. 698-705, 1997.

[11] K. H. Wesseling, N. smith, W. Nichols, H. Weber, B. de Wit, and J. E. Beneken, Beat-to-beat cardiac output from the arterial pressure pulse contour, ser. Boerhaave series for postgraduate medical education. Leiden: Leiden University Press, 1974, pp. 148-64.

[12] N. Westerhof, J.-W. Lankhaar, and B. E. Westerhof, "The arterial windkessel," Medical, biological engineering and computing, vol. 47, no. 2, pp. 131-41, 2009.

[13] J. M. Bland and D. G. Altman, "Statistical methods for assessing agreement between two methods of clinical measurement," Lancet, vol. 1, no. 8476, pp. 307-10, 1986.

[14] L. A. Critchley and J. A. Critchley, "A meta-analysis of studies using bias and precision statistics to compare cardiac output measurement techniques," J Clin Monit Comput, vol. 15, no. 2, pp. 85-91, 1999.

[15] M. Columb, "Clinical measurement and assessing agreement," Curr Anaesth Crit Care, no. 19, p. 2, 2008.

[16] L. A. Critchley, A. Lee, and A. M. Ho, "A critical review of the ability of continuous cardiac output monitors to measure trends in cardiac output," Anesthesia and analgesia, vol. 111, no. 5, pp. 1180-92, 2010.

[17] L. A. Critchley, X. X. Yang, and A. Lee, "Assessment of trending ability of cardiac output monitors by polar plot methodology," Journal of cardiothoracic and vascular anesthesia, vol. 25, no. 3, pp. 536-46, 2011. 
[18] A. f. t. a. o. m. instrumentation, "Electronic or automated sphygmomanometers," ANSI/AAMI SP10-1992. American National Standard Arlington, VA: AAMI, 1992.

[19] J. R. Martina, B. E. Westerhof, J. van Goudoever, E. M. de Beaumont, J. Truijen, Y. S. Kim, R. V. Immink, D. A. Jobsis, M. W. Hollmann, J. R. Lahpor, B. A. de Mol, and J. J. van Lieshout, "Noninvasive continuous arterial blood pressure monitoring with nexfin(r)," Anesthesiology, vol. 116, no. 5, pp. 1092-103, 2012.

[20] J. F. Stover, R. Stocker, R. Lenherr, T. A. Neff, S. R. Cottini, B. Zoller, and M. Bechir, "Noninvasive cardiac output and blood pressure monitoring cannot replace an invasive monitoring system in critically ill patients," BMC anesthesiology, vol. 9, p. 6, 2009.

[21] J. Akkermans, M. Diepeveen, W. Ganzevoort, G. A. van Montfrans, B. E. Westerhof, and H. Wolf, "Continuous non-invasive blood pressure monitoring, a validation study of nexfin in a pregnant population," Hypertens Pregnancy, vol. 28, no. 2, pp. 230-42, 2009.

[22] R. M. Nowak, A. Sen, A. J. Garcia, H. Wilkie, J. J. Yang, M. R. Nowak, and M. L. Moyer, "Noninvasive continuous or intermittent blood pressure and heart rate patient monitoring in the ed," The American journal of emergency medicine, vol. 29, no. 7, pp. 782-9, 2011.

[23] A. G. van der Spoel, A. J. Voogel, A. Folkers, C. Boer, and R. A. Bouwman, "Comparison of noninvasive continuous arterial waveform analysis (nexfin) with transthoracic doppler echocardiography for monitoring of cardiac output," Journal of clinical anesthesia, vol. 24, no. 4, pp. 304-9, 2012.

[24] O. Broch, J. Renner, M. Gruenewald, P. Meybohm, J. Schottler, A. Caliebe, M. Steinfath, M. Malbrain, and B. Bein, "A comparison of the nexfin(r) and transcardiopulmonary thermodilution to estimate cardiac output during coronary artery surgery,” Anaesthesia, vol. 67, no. 4, pp. 377-83, 2012.

[25] B. P. Imholz, W. Wieling, G. A. van Montfrans, and K. H. Wesseling, "Fifteen years experience with finger arterial pressure monitoring: assessment of the technology," Cardiovasc Res, vol. 38, no. 3, pp. 605-16, 1998.

[26] P. J. Peyton and S. W. Chong, "Minimally invasive measurement of cardiac output during surgery and critical care: a meta-analysis of accuracy and precision," Anesthesiology, vol. 113, no. 5, pp. 1220-35, 2010.

[27] J. M. Feldman, "Is it a bird? is it a plane? the role of patient monitors in medical decision making," Anesthesia and analgesia, vol. 108, no. 3, pp. 707-10, 2009. 


\title{
10
}

\section{Non-invasive measurement of pulse pressure variation and systolic pressure variation using a finger cuff corresponds with intra-arterial measurement}

\author{
B. Lansdorp, D. Ouweneel, A. de Keijzer, J. G. van der Hoeven, J. Lemson and P. \\ Pickkers. \\ British Journal of Anaesthesia 2011 Oct;107(4):540-5
}

Background. Pulse pressure variation $(P P V)$ and systolic pressure variation $(S P V)$ are reliable predictors of fluid responsiveness in patients undergoing controlled mechanical ventilation. Currently, $P P V$ and $S P V$ are measured invasively and it is unknown if an arterial pressure (AP) signal obtained with a finger cuff can be used as an alternative. The aim of this study was to validate $P P V$ and $S P V$ measured using a finger cuff.

Methods. Patients receiving mechanical ventilation under sedation after cardiac artery bypass graft (CABG) surgery were included after arrival on the intensive care unit. AP was measured invasively in the radial artery and non-invasively using the finger cuff of the NexfinTM monitor. I.V. fluid challenges were administered according to clinical need. The mean value of $P P V$ and SPV was calculated before and after administration of a fluid challenge. Agreement of the calculated $P P V$ and $S P V$ from both methods was assessed using the BlandAltman analysis.

Results. Nineteen patients were included and 28 volume challenges were analysed. Correlation between the two methods for $P P V$ and $S P V$ [mean $(\mathrm{SD})=6.9(4.3) \%$ and $5.3(2.6) \%$, respectively] was $\mathrm{r}=0.96$ 
$(\mathrm{P}<0.001)$ and $\mathrm{r}=0.95(\mathrm{P}<0.001)$, respectively. The mean bias was $20.95 \%$ for $P P V$ and $20.22 \%$ for $S P V$. Limits of agreement were $24.3 \%$ and $2.4 \%$ for $P P V$ and $22.2 \%$ and $1.7 \%$ for $S P V$. The correlation between changes in $P P V$ and $S P V$ as a result of volume expansion measured by the two different methods was $\mathrm{r}=0.88(\mathrm{P}<0.001)$ and $\mathrm{r}=0.87(\mathrm{P}<0.001)$, respectively.

Conclusions. In patients receiving controlled mechanical ventilation after CABG, $P P V$ and $S P V$ can be measured reliably non-invasively using the inflatable finger cuff of the NexfinTM monitor. 


\subsection{Introduction}

I.V. volume expansion is frequently used in critically ill patients to improve tissue perfusion, but overzealous fluid administration may also be detrimental [1-4]. Several predictors of fluid responsiveness are available to identify patients who may benefit from i.v. volume expansion indicated by an increase in cardiac stroke volume (responders) and to prevent hypervolaemia in patients who do not (nonresponders). Among these, several studies have shown that dynamic indices are more reliable than traditional measures (e.g. central venous pressure and pulmonary capillary wedge pressure) to guide volume resuscitation in patients undergoing controlled mechanical ventilation [5-7]. Dynamic indices quantify the cyclic changes in left and right ventricular preload and afterload [8] by measuring, for example, the variation in pulse pressure (pulse pressure variation, $P P V$ ) or systolic pressure (systolic pressure variation, $S P V)$. Patients whose cardiac function is operating on the steep portion of the FrankStarling curve are more likely to show an increase in these arterial pressure (AP) variations and benefit from i.v. fluid administration [9].

Although dynamic indices such as $P P V$ and $S P V$ have proven their predictive value, they depended on direct intra-AP monitoring, which is a relative drawback. The ability to monitor $P P V$ and $S P V$ non-invasively may increase their clinical usefulness in patients without an arterial catheter, for example, in acute situations in the emergency room.

The NexfinTM monitor (BMEYE, Amsterdam, The Netherlands) provides a continuous AP measurement using a finger cuff, based on the principle introduced by Penaz and colleagues[10]. The method is based on the development of the dynamic (pulsatile) unloading of the finger arterial walls using an inflatable finger cuff with a built-in photo-electric plethysmograph [11-13]. In addition, the Nexfin monitor reconstructs the finger AP wave to a brachial artery wave by application of a filter [14] which increases reliability in reflecting true AP [15].

The aim of this study was to evaluate the reliability of $P P V$ and $S P V$, calculated from the finger AP and the reconstructed brachial pressure in comparison with the gold standard of direct intra-AP measurement.

\subsection{Methods}

\subsubsection{Patients}

Because of the observational and non-invasive nature of this study, the local medical ethics board waived the need for informed consent. Twenty patients undergoing controlled mechanical ventilation after cardiac artery bypass graft (CABG) surgery were prospectively studied from the time of admission to the intensive care unit (ICU). Fluid challenges were administered by the attending physician based upon the pres- 


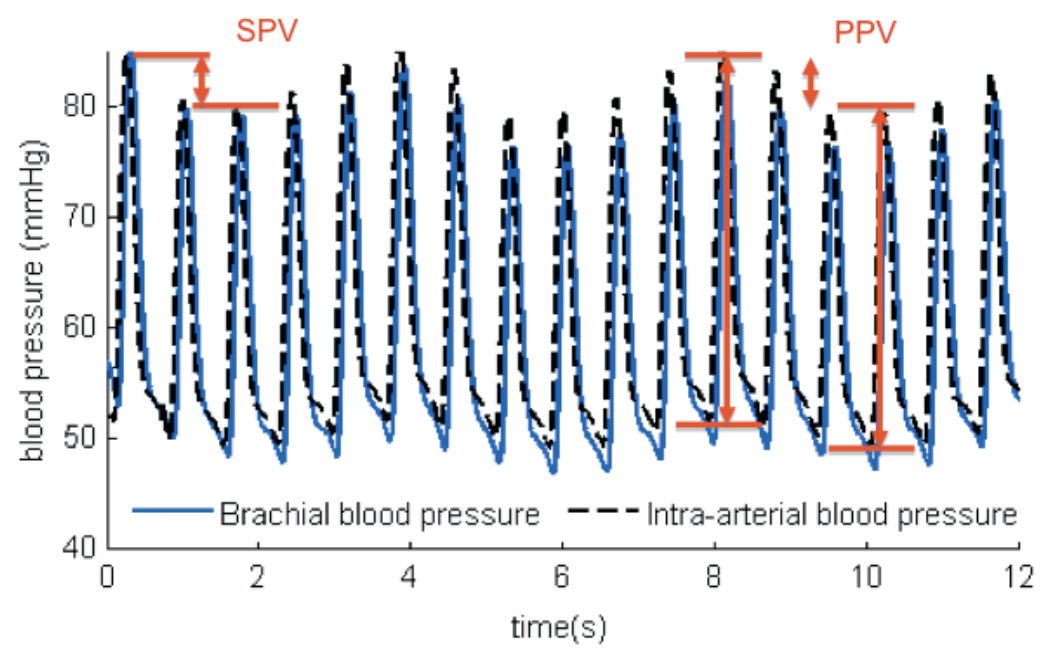

Figure 1: Example of simultaneous recording of the intra-arterial $A P\left(A P_{I A}\right)$ and brachial $A P\left(A P_{B R A C H}\right)$, with illustrated SPV and PPV.

ence of at least one clinical sign of inadequate tissue perfusion [low mean AP (MAP), low urine production, cold and clammy extremities, increased lactate level, or low central venous oxygen saturation]. We excluded patients who did not receive at least one fluid challenge, were showing spontaneous respiratory efforts, and those with an intra-aortic balloon pump.

\subsubsection{Haemodynamic monitoring}

In all patients, a central venous catheter was inserted into the internal jugular vein. Mechanical ventilation of the patients lungs was performed using a Servo 300 ventilator (Maquet, Rasstat, Germany). Administration of vasoactive medications was guided by a standard clinical protocol.

AP was monitored invasively $\left(A P_{I A}\right)$ using a $20 \mathrm{G}$ radial artery catheter connected via standard low compliant tubing to a disposable pressure transducer (Edwards Lifesciences, Irvine, CA, USA). The intra-arterial signal was registered using a HP monitor (Merlin M1046A, Hewlett Packard, USA). The non-invasive arterial signal was obtained using a finger cuff adjusted to the size of the index finger of the patient according to the guidelines of the manufacturer and connected to the NexfinTM monitor [BMEYE NexfinTM Monitor (BMEYE, Amsterdam, the Netherlands)]. This noninvasive measurement provides the AP in the finger arteries $\left(A P_{F I N G}\right)$ and from this signal, the reconstructed AP in the brachial artery $\left(A P_{B R A C H}\right)$ is calculated. All three signals were recorded simultaneously using a sample rate of $200 \mathrm{~Hz}$ and stored on 
a hard disk. Figure 1 shows an example of the simultaneous recordings of the AP, together with a representation of the $P P V$ and $S P V$.

From all the recorded waveforms, $P P V$ and $S P V$ were calculated offline using the mathematical computer program Matlab (Matlab R2009b, MathWorks Inc., MA, USA) using the following formula:

$$
\begin{aligned}
& P P V=\frac{\text { PPmax }- \text { PPmin }}{\text { PPmean }} \\
& S P V=\frac{\text { SPmax }- \text { SPmin }}{\text { SPmean }}
\end{aligned}
$$

where $P P$ and $S P$ are the pulse pressure (systolic minus diastolic pressure) and systolic pressure, respectively. The subscripts max, min, and mean indicate, respectively, the average values of the four maximum, the four minimum, and all pulse pressures or systolic pressures during 30s. This technique is also used by Pulsion (Pulsion Medical Systems, Munich, Germany) and Drager (Drager Medical, Lubech, Germany) to calculate dynamic indices in the absence of respiratory data. The indices were detected automatically by an algorithm written in Matlab and were visually inspected offline for errors.

\subsubsection{Design}

To compare the calculation of the $P P V$ and $S P V$ from the noninvasive signal ( $P P V_{F I N G}$ $/ P P V_{B R A C H}$ and $\left.S P V_{F I N G} / S P V_{B R A C H}\right)$ with the signal from the intra-arterial catheter $\left(P P V_{I A}\right.$ and $\left.S P V_{I A}\right)$, measurements of AP were performed before and after each fluid challenge (130/0.4 6\% HES solution Voluven, Frensenius Kabi, s-Hertogenbosch, the Netherlands) administered according to instructions of the attending physician. Infusion time and volume were registered.

The baseline measurement was performed over 30s within the last minute before the start of the fluid challenge. The second measurement (after VE) was performed within $3 \mathrm{~min}$ after the end of the infusion. To investigate the accuracy of the non-invasive method to monitor changes of the haemodynamic parameters over time, the change in $P P V$ and $S P V$ as a result of the fluid challenge $(\triangle P P V, \triangle S P V)$ measured non-invasively was compared with the response calculated from the intraarterial measurement.

\subsubsection{Statistical analysis}

Haemodynamic measurements are reported as mean [standard deviation (SD)]. Agreement between the two methods was assessed using the method described by Bland and Altman [16]. The correlation between $P P V_{F I N G} / P P V_{B R A C H}$ and $P P V_{I A}$ and be- 
tween $S P V_{F I N G} / S P V_{B R A C H}$ and $S P V_{I A}$ was calculated using the Pearson correlation coefficient (r) after normality was checked and corrected for repeated measurements [17]. To detect a correlation of at least 0.7 with a power of $90 \%$ and a P-value of 0.05 , at least 18 individual measurements were needed. We therefore included 20 patients with the possibility of multiple measurements per patient. In addition, the bias (the difference between the two methods given by $P P V_{F I N G} / P P V_{B R A C H}-P P V_{I A}$ and $\left.S P V_{F I N G} / S P V_{B R A C H}-S P V_{I A}\right)$ was calculated and plotted against the average of the two different methods. Limits of agreement were calculated using the standard deviation of the differences (s) (d21.96 s and d+1.96 s), also corrected for multiple observations per individual [16]. Finally, the standard errors and confidence intervals were calculated. Statistical analysis was done using SPSS Statistics 18.0 for windows (SPSS Inc., Chicago, IL, USA).

\subsection{Results}

A total of 30 fluid challenges were administered. Two fluid challenges were excluded because AP variations could not reliably be calculated, one because of arrhythmias and one because of a distorted signal due to incorrect placement of the finger cuff. Table 1 shows the characteristics of the remaining 19 patients and 28 fluid challenges. A total of 56 simultaneous $P P V$ and $S P V$ measurements were available for final analysis. A strong correlation was found between the dynamic indices derived from the intra-arterial waveform and those from the finger and reconstructed brachial waveform, for both the $P P V$ and the $S P V$ (Figure 2). Figure 2 also shows that both $S P V_{B R A C H}$ and $P P V_{B R A C H}$ have only a few false positives and false negatives taking into account the previously published cut-off values regarding their predictive value for fluid responsiveness: $7 \%$ and $12 \%$ for $S P V$ and $P P V, 6$ respectively. This results in a sensitivity and specificity of $92 \%$ and $96 \%$ for $S P V$ and $100 \%$ and $94 \%$ for $P P V$.

The mean (absolute) bias compared with the intra-arterial measurement was $21.0 \%$ and $20.2 \%$ for $P P V_{B R A C H}$ and $S P V_{B R A C H}$, respectively. For $P P V_{F I N G}$ and $S P V_{F I N G}$, the mean bias was $20.74 \%$ and $20.11 \%$, respectively. The mean bias, including the relative bias, and the related limits of agreement are also shown in Table 2. The BlandAltman plot of the absolute values of $P P V_{I A}$ and $P P V_{B R A C H}$ and $S P V_{I A}$ and $S P V_{B R A C H}$ is shown in Figure 3. There was no relationship between the difference and the mean value of the two measurement methods.

To study the ability of the non-invasive method to monitor changes of $P P V$ and $S P V$ over time, the change in $P P V$ and $S P V(\triangle P P V$ and $\triangle S P V$ ) caused by volume expansion was calculated for the measurement with the arterial catheter and the finger cuff. Correlation between $\triangle P P V$ and $\triangle S P V$ measured from the intra-arterial signal $\left(\triangle P P V_{I A}\right.$ and $\left.\triangle S P V_{I A}\right)$ and from the non-invasively measured reconstructed brachial pressure $\left(\triangle P P V_{B R A C H}\right.$ and $\left.\triangle S P V_{B R A C H}\right)$ was 0.88 and 0.87 for $P P V$ and $S P V$, respec- 

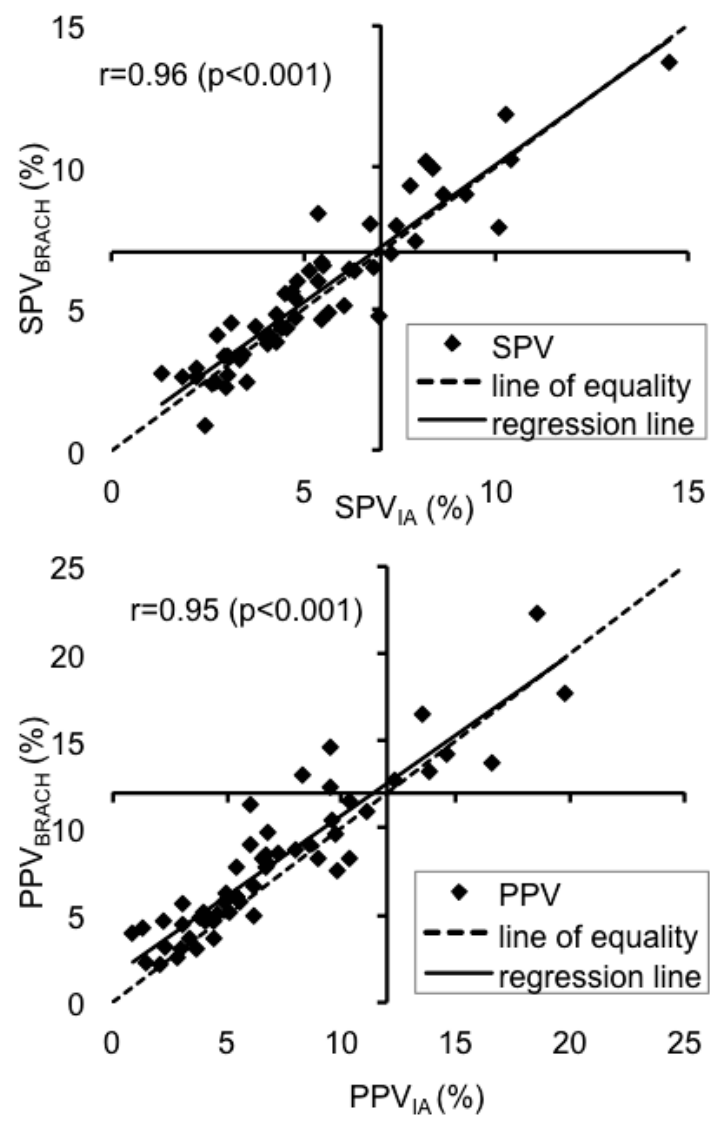

Figure 2: Correlation between $S P V(A)$ and $P P V(B)$ measured from the intra-arterial signal $\left(S P V_{I A}\right.$ and $\left.P P V_{I A}\right)$ and from the noninvasively measured reconstructed brachial pressure $\left(S P V_{B R A C H}\right.$ and $\left.P P V_{B R A C H}\right)$. Taking into account the previously published cut-off values for $S P V$ and PPV regarding their predictive value for fluid responsiveness ( 7 and 12\%, respectively, see axes), both $S P V_{B R A C H}$ and $P P V_{B R A C H}$ have only a few false positives and false negatives. This results in a sensitivity and specificity of $92 \%$ and $96 \%$ for SPV and $100 \%$ and $94 \%$ for $P P V$, respectively. 

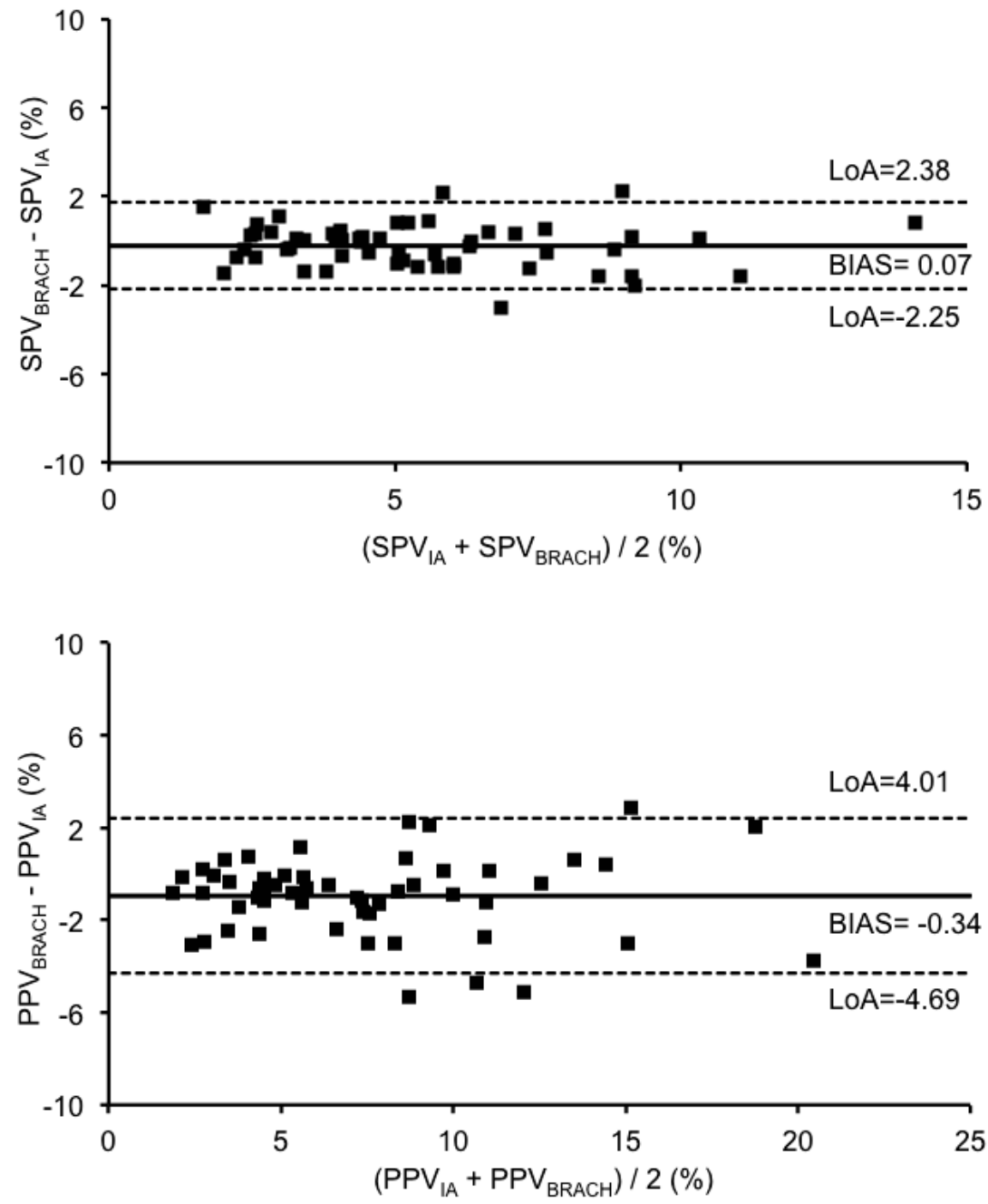

Figure 3: Mean bias [BIAS (\%), solid line] and limits of agreement [LoA (\%), dashed line] according to the BlandAltman analyses. Comparison of the SPV $(A)$ and PPV (B) measured from the intra-arterial signal $\left(S P V_{I A}\right.$ and $\left.P P V_{I A}\right)$ and from the noninvasively measured reconstructed brachial pressure ( $S P V_{B R A C H}$ and $\left.P P V_{B R A C H}\right)$. 

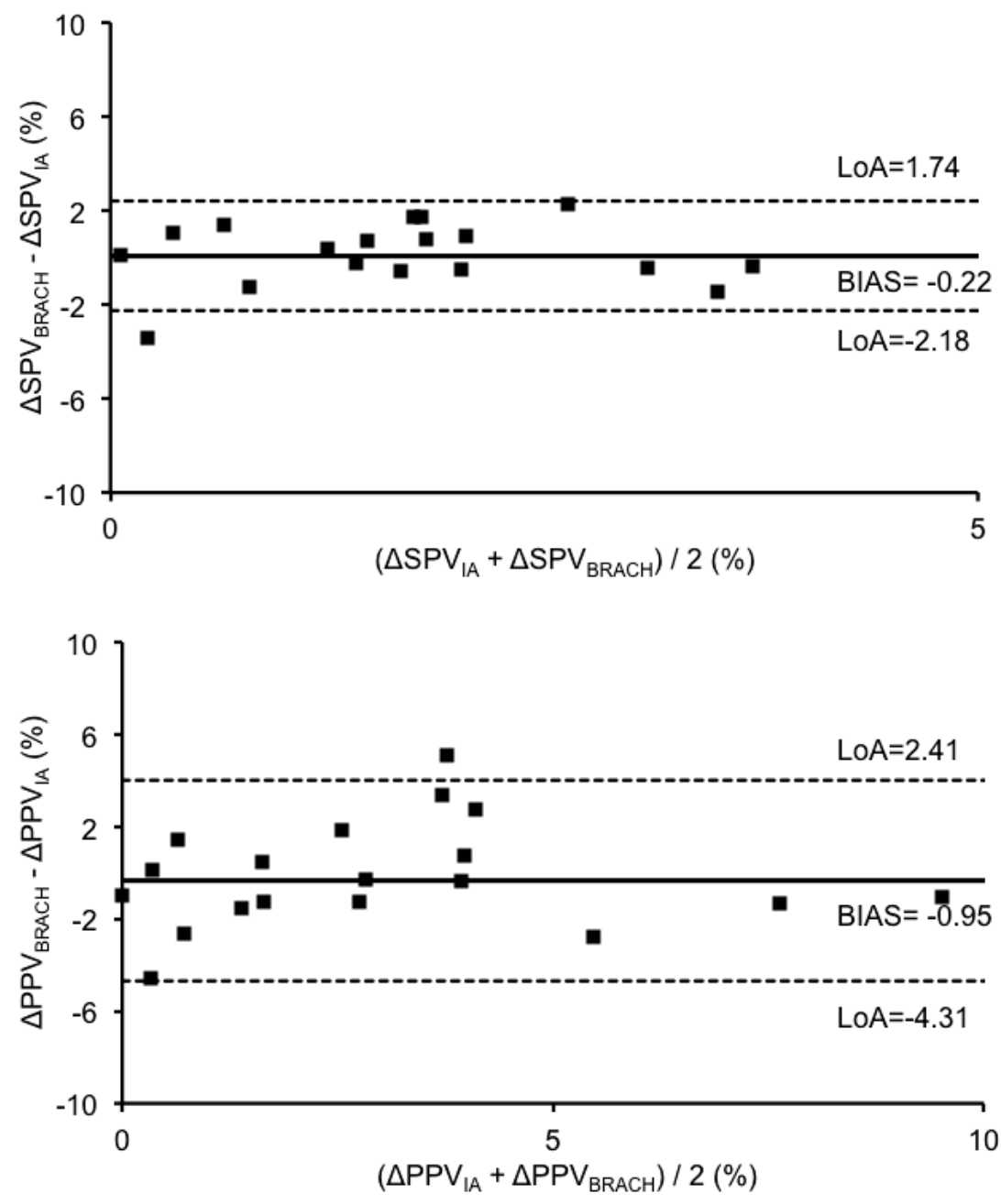

Figure 4: Mean bias [BIAS (\%), solid line] and limits of agreement [LoA (\%), dashed line] according to the BlandAltman analyses. Comparison of the change in SPV (A) and $P P V(B)$ as a result of the fluid challenge, measured from the intra-arterial signal $\left(\% S P V_{I A}\right.$ and $\left.\% P P V_{I A}\right)$ and from the non-invasively measured reconstructed brachial pressure $\left(\% S P V_{B R A C H}\right.$ and $\left.\% P P V_{B R A C H}\right)$. 
Table 1: Patients characteristics and baseline haemodynamic and respiratory variables, presented as mean (range), mean (SD) or number.

\begin{tabular}{||l|l||}
\hline \multicolumn{2}{||c||}{ patient characteristics \& baseline parameters } \\
\hline Patient $(\#)$ & 19 \\
Male/Female (\#) & $14 / 5$ \\
Age $(\mathrm{yr})$ & $66(58-79)$ \\
Weight $(\mathrm{kg})$ & $78.9(15.0)$ \\
Heart rate $\left(\right.$ beats $\left.\cdot \mathrm{min}^{-1}\right)$ & $66.0(14.8)$ \\
Mean arterial pressure $(\mathrm{mmHg})$ & $70.0(5.9)$ \\
Central venous pressure $(\mathrm{mmHg})$ & 10.1 \\
Tidal volume $\left(\mathrm{ml} \cdot \mathrm{kg}_{1} \mathrm{IBW}\right)$ & $7.6(1.0)$ \\
Ventilatory frequency $(\mathrm{bpm})$ & $12.1(1.4)$ \\
PEEP $\left(\mathrm{cmH} \mathrm{H}_{2} \mathrm{O}\right)$ & $5.7(2.0)$ \\
Plateau pressure $\left(\mathrm{cm} \mathrm{H}_{2} \mathrm{O}\right)$ & $16.9(2.7)$ \\
\hline \multicolumn{2}{|c|}{ Intervention } \\
\hline Number of fluid challenges $(\#)$ & 28 \\
Infusion volume $(\mathrm{ml})$ & $390(130)$ \\
Infusion duraion $(\mathrm{min})$ & $7.6(6.5)$ \\
\hline
\end{tabular}

tively (both $\mathrm{P}, 0.001$ ). The corresponding BlandAltman plots are shown in Figure 4. Correlation coefficients, including the mean bias and limits of agreement, are all displayed in Table2. No significant differences were observed while comparing the correlation coefficients of $S P V_{F I N G}$ and $S P V_{B R A C H}$ or $P P V_{F I N G}$ and $P P V_{B R A C H}$.

\subsection{Discussion}

This study indicates that the respiratory variations in pulse pressure and systolic pressure can be calculated reliably using a non-invasive finger AP measurement. Therefore, an arterial line may not be necessary to assess fluid responsiveness using dynamic indices. Only one other study describes the calculation of dynamic indices from the finger AP obtained non-invasively [18]. In accordance with the results of this study, we found a small mean bias with similar limits of agreement for the $P P V_{F I N G}$ compared with the $P P V_{I A}$. In addition, we used the reconstructed brachial pressure for the calculation of $P P V$ and $S P V$. The resultant waveform of this signal is more similar to the invasively measured pressure in the radial artery and the reliability was further improved when the reconstructed brachial pressure signal was used, illustrated by a slightly higher correlation coefficient and narrower limits of agreement. 
Table 2: Correlation coefficients and the BlandAltman variables for the SPV and PPV measured from the non-invasively measured reconstructed brachial pressure ( $S P V_{B R A C H}$ and $\left.P P V_{B R A C H}\right)$ and the non-invasively measured finger pressure (SPVFING and $\left.P P V_{F I N G}\right)$, both compared with the SPV and PPV measured from the intra-arterial signal ( $S P V_{I A}$ and $\left.P P V_{I A}\right)$. Correlation coefficients were significant $(*=P<0.001)$. No significant differences were observed while comparing the correlation coefficients of $S P V_{F I N G}$ and $S P V_{B R A C H}$ or PPV FING and $P P V_{B R A C H}$.

\begin{tabular}{|c|c|c|c|c|}
\hline & $P P V_{B R A C H}$ & $P P V_{F I N G}$ & $S P V_{B R A C H}$ & $S P V_{F I N G}$ \\
\hline \multicolumn{5}{|c|}{ Absolute value of PPV/SPV } \\
\hline Correlation coefficient (r) & $0.96^{*}$ & $0.94 *$ & $0.95^{*}$ & $0.93 *$ \\
\hline Absolute mean bias (\%) & -0.95 & -0.74 & -0.22 & -0.11 \\
\hline Relative mean bias (\%) & -12.9 & -10.1 & -4.1 & -2.0 \\
\hline Limits of agreement (\%) & \pm 3.36 & \pm 3.99 & \pm 1.96 & \pm 2.47 \\
\hline \multicolumn{5}{|l|}{ Change in PPV/SPV } \\
\hline Correlation coefficient $(\mathrm{r})$ & $0.88^{*}$ & $0.82 *$ & $0.87 *$ & $0.78^{*}$ \\
\hline Absolute mean bias (\%) & -0.33 & -0.21 & 0.07 & 0.31 \\
\hline Relative mean bias (\%) & $0.96^{*}$ & $0.94 *$ & $0.95 *$ & $0.93^{*}$ \\
\hline Limits of agreement (\%) & \pm 4.35 & \pm 5.03 & \pm 2.32 & \pm 3.04 \\
\hline
\end{tabular}

Over the past few years, other non-invasively obtained dynamic indices have been suggested as predictors for volume responsiveness, including pulse oximetry wave variation and the cyclic changes derived by Doppler echocardiography. In comparison with our results, the respiratory variations in pulse oximetry plethysmographic waveform amplitude show a less strong correlation coefficient with arterial PPV $[19,20]$ and wider limits of agreement $[18,19]$. Respiratory variations in the diameter of the inferior vena cava visualized by echocardiography predict volume responsiveness with a positive predictive value of $93 \%$ and a negative predictive value of $92 \%[21,22]$. Sensitivity and specificity for variation of peak aortic blood velocity were $100 \%$ and $89 \%$, respectively [23, 24]. Although these results are comparable with $P P V$ and $S P V$ determined via an arterial line or non-invasively as in our study, limitations of echo(cardio)graphy include high costs, training, and workload to perform the measurements.

Measuring the $P P V$ and $S P V$ without the need of an intra-arterial catheter is an advantage for patients undergoing surgery or in the emergency room who are undergoing mechanical ventilation but without an intra-arterial catheter in situ. The intra-arterial catheter waveform and subsequent pressure calculations are considered the gold standard. However, errors may occur resulting from both the catheter and fluid-filled tubing that transfers the pressure signal, while the finger pressure cuff has a more direct relation to the finger artery. Theoretically, the finger AP measurement 
may be more reliable on some occasions, but clinical experience is scarce.

It may also be useful to assess AP variations in patients breathing spontaneously. Recent findings show that dynamic indices can predict volume responsiveness in spontaneous breathing patients whether or not in combination with the passive leg raising test or the Valsalva manoeuvre [25-27]. Although we investigated the validity of $P P V$ and $S P V$ in patients undergoing mechanical ventilation, we hypothesize that the origin (positive or negative airway pressure) of the AP variation does not influence the accuracy of the non-invasive measurement method.

We appreciate that there are several limitations in our study. First, because of the observational character of this study, we did not measure cardiac output. As it was not the aim of this study to confirm that dynamic indices predict fluid responsiveness as determined by an increase in cardiac output, we cannot draw any conclusions from our results about the predictive value regarding fluid responsiveness using the dynamic indices derived from the finger cuff. However, the predictive value of dynamic indices has been demonstrated previously and we found that noninvasive measurement had a high sensitivity and specificity in distinguishing presumed responders and non-responders defined by indices measured invasively. However, it is important to realize that three patients would have been misclassified using the non-invasive system and further data are required for clinical validation. Secondly, the 56 measurements that we used for comparison of the various methods were derived from 28 fluid challenges in 19 patients. To overcome this issue, we corrected the statistics for repeated measurements per individual. Another potential limitation is the use of cardiac surgery patients, since they might not be representative for standard more general ICU population. Especially, since there have been concerns about the use of the Penaz techniques in general critical care patients with poor peripheral perfusion [28]. Nevertheless, in recent years, this technique has significantly been improved and appears also applicable in the critically ill [29].

In conclusion, $P P V$ and $S P V$ can be measured reliably in a non-invasive manner using a non-invasive finger AP measurement in ICU patients on controlled mechanical ventilation after CABG surgery.

\section{References}

[1] D. Payen, A. C. de Pont, Y. Sakr, C. Spies, K. Reinhart, and J. L. Vincent, “A positive fluid balance is associated with a worse outcome in patients with acute renal failure," Crit Care, vol. 12, no. 3, p. R74, 2008.

[2] J. L. Vincent and M. H. Weil, "Fluid challenge revisited," Crit Care Med, vol. 34, no. 5, pp. 1333-7, 2006.

[3] S. Brandt, T. Regueira, H. Bracht, F. Porta, S. Djafarzadeh, J. Takala, J. Gorrasi, 
E. Borotto, V. Krejci, L. B. Hiltebrand, L. E. Bruegger, G. Beldi, L. Wilkens, P. M. Lepper, U. Kessler, and S. M. Jakob, "Effect of fluid resuscitation on mortality and organ function in experimental sepsis models," Crit Care, vol. 13, no. 6, p. R186, 2009.

[4] A. L. Rosenberg, R. E. Dechert, P. K. Park, and R. H. Bartlett, "Review of a large clinical series: association of cumulative fluid balance on outcome in acute lung injury: a retrospective review of the ardsnet tidal volume study cohort," $J$ Intensive Care Med, vol. 24, no. 1, pp. 35-46, 2009.

[5] F. Michard and J. L. Teboul, "Predicting fluid responsiveness in icu patients: a critical analysis of the evidence," Chest, vol. 121, no. 6, pp. 2000-8, 2002.

[6] F. Michard, S. Boussat, D. Chemla, N. Anguel, A. Mercat, Y. Lecarpentier, C. Richard, M. R. Pinsky, and J. L. Teboul, "Relation between respiratory changes in arterial pulse pressure and fluid responsiveness in septic patients with acute circulatory failure," Am J Respir Crit Care Med, vol. 162, no. 1, pp. $134-8,2000$.

[7] A. Kramer, D. Zygun, H. Hawes, P. Easton, and A. Ferland, "Pulse pressure variation predicts fluid responsiveness following coronary artery bypass surgery," Chest, vol. 126, no. 5, pp. 1563-8, 2004.

[8] F. Michard, "Changes in arterial pressure during mechanical ventilation," Anesthesiology, vol. 103, no. 2, pp. 419-28, 2005.

[9] F. Feihl and A. F. Broccard, "Interactions between respiration and systemic hemodynamics. part i: basic concepts," Intensive Care Med, vol. 35, no. 1, pp. 45-54, 2009.

[10] J. Penaz, A. Voigt, and W. Teichmann, "[contribution to the continuous indirect blood pressure measurement]," Z Gesamte Inn Med, vol. 31, no. 24, pp. 1030-3, 1976.

[11] B. P. Imholz, W. Wieling, G. A. van Montfrans, and K. H. Wesseling, "Fifteen years experience with finger arterial pressure monitoring: assessment of the technology," Cardiovasc Res, vol. 38, no. 3, pp. 605-16, 1998.

[12] J. Akkermans, M. Diepeveen, W. Ganzevoort, G. A. van Montfrans, B. E. Westerhof, and H. Wolf, "Continuous non-invasive blood pressure monitoring, a validation study of nexfin in a pregnant population," Hypertens Pregnancy, vol. 28, no. 2, pp. 230-42, 2009. 
[13] D. W. Eeftinck Schattenkerk, J. J. van Lieshout, A. H. van den Meiracker, K. R. Wesseling, S. Blanc, W. Wieling, G. A. van Montfrans, J. J. Settels, K. H. Wesseling, and B. E. Westerhof, "Nexfin noninvasive continuous blood pressure validated against riva-rocci/korotkoff," Am J Hypertens, vol. 22, no. 4, pp. 37883, 2009.

[14] W. J. Bos, J. van Goudoever, G. A. van Montfrans, A. H. van den Meiracker, and K. H. Wesseling, "Reconstruction of brachial artery pressure from noninvasive finger pressure measurements," Circulation, vol. 94, no. 8, pp. 1870-5, 1996.

[15] P. Gizdulich, B. P. Imholz, A. H. van den Meiracker, G. Parati, and K. H. Wesseling, "Finapres tracking of systolic pressure and baroreflex sensitivity improved by waveform filtering," J Hypertens, vol. 14, no. 2, pp. 243-50, 1996.

[16] J. M. Bland and D. G. Altman, "Agreement between methods of measurement with multiple observations per individual," J Biopharm Stat, vol. 17, no. 4, pp. 571-82, 2007.

[17] _ - "Calculating correlation coefficients with repeated observations: Part 2correlation between subjects," BMJ, vol. 310, no. 6980, p. 633, 1995.

[18] H. Solus-Biguenet, M. Fleyfel, B. Tavernier, E. Kipnis, J. Onimus, E. Robin, G. Lebuffe, C. Decoene, F. R. Pruvot, and B. Vallet, "Non-invasive prediction of fluid responsiveness during major hepatic surgery," Br J Anaesth, vol. 97, no. 6, pp. 808-16, 2006.

[19] M. Cannesson, C. Besnard, P. G. Durand, J. Bohe, and D. Jacques, "Relation between respiratory variations in pulse oximetry plethysmographic waveform amplitude and arterial pulse pressure in ventilated patients," Crit Care, vol. 9, no. 5, pp. R562-8, 2005.

[20] G. A. Westphal, E. Silva, A. R. Goncalves, M. Caldeira Filho, and L. F. Polide Figueiredo, "Pulse oximetry wave variation as a noninvasive tool to assess volume status in cardiac surgery," Clinics (Sao Paulo), vol. 64, no. 4, pp. 337 43, 2009.

[21] C. Barbier, Y. Loubieres, C. Schmit, J. Hayon, J. L. Ricome, F. Jardin, and A. Vieillard-Baron, "Respiratory changes in inferior vena cava diameter are helpful in predicting fluid responsiveness in ventilated septic patients," Intensive Care Med, vol. 30, no. 9, pp. 1740-6, 2004.

[22] M. Feissel, F. Michard, J. P. Faller, and J. L. Teboul, "The respiratory variation in inferior vena cava diameter as a guide to fluid therapy," Intensive Care Med, vol. 30, no. 9, pp. 1834-7, 2004. 
[23] M. Feissel, F. Michard, I. Mangin, O. Ruyer, J. P. Faller, and J. L. Teboul, "Respiratory changes in aortic blood velocity as an indicator of fluid responsiveness in ventilated patients with septic shock," Chest, vol. 119, no. 3, pp. 867-73, 2001.

[24] A. Vieillard-Baron, K. Chergui, R. Augarde, S. Prin, B. Page, A. Beauchet, and F. Jardin, "Cyclic changes in arterial pulse during respiratory support revisited by doppler echocardiography," Am J Respir Crit Care Med, vol. 168, no. 6, pp. 671-6, 2003.

[25] M. I. Monge Garcia, A. Gil Cano, and J. C. Diaz Monrove, "Arterial pressure changes during the valsalva maneuver to predict fluid responsiveness in spontaneously breathing patients," Intensive Care Med, vol. 35, no. 1, pp. 77-84, 2009.

[26] S. Soubrier, F. Saulnier, H. Hubert, P. Delour, H. Lenci, T. Onimus, S. Nseir, and A. Durocher, "Can dynamic indicators help the prediction of fluid responsiveness in spontaneously breathing critically ill patients?" Intensive Care Med, vol. 33, no. 7, pp. 1117-24, 2007.

[27] R. Skulec, O. Cermak, H. Skalicka, and J. Kolar, "Variability of aortic blood flow predicts fluid responsiveness in spontaneously breathing healthy volunteers," Kardiol Pol, vol. 67, no. 3, pp. 265-71, 2009.

[28] I. K. Farquhar, "Continuous direct and indirect blood pressure measurement (finapres) in the critically ill," Anaesthesia, vol. 46, no. 12, pp. 1050-5, 1991.

[29] K. van de Vijver, A. Verstraeten, C. Gillebert, U. Maniewski, M. Gabrovska, D. Viskens, N. van Regenmortel, I. de Laet, K. Schoonheydt, H. Dits, and M. Malbrain, "Validation of non-invasive hemodynamic monitoring with nexfin in critically ill patients," p. 15(Suppl 1):P75, 22-25 March 20112011. 


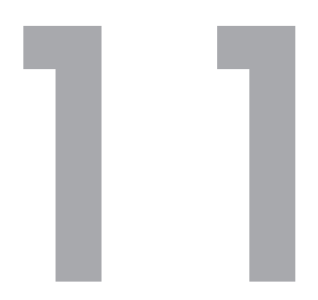

\section{Non-invasive determination of fluid responsiveness using dynamic indices and the passive leg-raising test}

C.Hofhuizen, B. Lansdorp, P. Pickkers, M.J.A.M. van Putten, J.G. van der Hoeven, G.J. Scheffer and J. Lemson.

Submitted: Anesthesia \& Analgesia

\footnotetext{
Background. Dynamic indices and the response of cardiac output (CO) to a passive leg-raising (PLR) test adequately predict fluid responsiveness. However, both techniques require invasive monitoring. The aim of this study was to determine the ability of non-invasively derived dynamic indices and the PLR-test using the Nexfin to predict fluid responsiveness.

Methods. Nineteen post-cardiac surgery patients were studied during volume therapy. Arterial pressure $(\mathrm{ABP})$ and $\mathrm{CO}$ were monitored invasively intra-arterially and using the PiCCO system $\left(\mathrm{ABP}_{I A}\right.$ and $\mathrm{CO}_{T D}$ ), and non-invasively using the Nexfin $\left(\mathrm{ABP}_{N I}\right.$ and $\left.\mathrm{CO}_{N I}\right)$. Dynamic indices (pulse pressure-, systolic pressure- and stroke volume variation) and stroke volume index (SVI) response to a PLR-test prior to every fluid challenge were determined.

Results. In total, 27 fluid challenges were recorded, of which 14 resulted in a change in SVI $>12 \%$. Mean bias and limits of agreement were $-0.8 \%$ (-3.7\% to $2.1 \%$ ), $-0.1 \%$ (1.3\% to $1.0 \%$ ) and $-0.4 \%$ ($4.5 \%$ to $3.7 \%$ ) for PPV, SPV and SVV, respectively. The area under the curve was $0.78,0.55$ and 0.83 for $\mathrm{PPV}_{N I}, \mathrm{SPV}_{N I}$ and $\mathrm{SVV}_{N I}$, respectively. Concordance between changes in $\mathrm{SVI}_{N I}$ during PLR and changes in $\mathrm{SVI}_{T D}$ after a fluid challenge was $96 \%$. AUC of the PLR-induced change in CO was 0.64 and 0.68 for $\mathrm{SVI}_{N I}$ and $\mathrm{SNI}_{I A}$ respectively.
} 
Conclusions. Nexfin is a safe technique for tracking $\mathrm{CO}$ changes in cardiac surgery patients and predicting fluid responsiveness using dynamic indices. However, PLR using Nexfin cannot predict fluid responsiveness reliably. However, non-invasively derived dynamic indices and response to PLR-test are interchangeable with their invasive alternatives. 


\subsection{Introduction}

Fluid resuscitation is considered the first choice of therapy to improve haemodynamics in patients with a critically compromised circulation. However, only approximately half of the admitted fluid challenges result in an significant increase in cardiac output [1], and excessive fluid resuscitation is associated with increased morbidity and mortality [2]. For this reason, accurate prediction of fluid responsiveness is of paramount relevance in daily clinical practice. It has now become clear that dynamic indices including systolic pressure variation (SPV), pulse pressure variation (PPV) and stroke volume variation (SVV), in contrast to static preload markers like central venous pressure and pulmonary artery occlusion pressure [3], are able to predict fluid responsiveness accurately [4]. Unfortunately, dynamic indices are only reliable in patients without spontaneous breathing activity, ventilated with tidal volumes $>8 \mathrm{ml} / \mathrm{kg}$ and without cardiac arrhythmias [5]. In other situations, an increase in cardiac output in response to a passive leg-raising test is a good alternative with high sensitivity and specificity [6]. However, both dynamic indices and passive leg-raising need invasive monitoring, potentially leading to patient discomfort and complications. Current non-invasive haemodynamic devices including the Nexfin monitor (BMEYE, Amsterdam, The Netherlands) provide a continuous arterial pressure and cardiac output measurement using a finger cuff $[7,8]$. This makes it possible to track changes in $\mathrm{CO}$ during passive leg-raising and to derive dynamic indices from this signal [9]. The aim of the present study was to determine whether fluid responsiveness could be predicted non-invasively with the Nexfin monitor using dynamic indices and the PLR-test. We compared this performance with dynamic indices derived from an intra-arterial catheter and the transpulmonary thermodilution derived cardiac output changes during a PLR-test.

\subsection{Materials and Methods}

\subsubsection{Patients}

With the approval of the institutional review board and after obtaining participants informed consent, 20 patients were studied following cardiac surgery. Exclusion criteria were recent myocardial infarction and preoperative inotropic or intra-aortic balloon pump support. Patients that did not receive a fluid challenge during their ICU admission were excluded from analysis afterwards.

\subsubsection{Haemodynamic monitoring}

A central venous catheter was inserted in the internal jugular vein for the measurement of the central venous pressure (CVP). Arterial blood pressure (ABP) was mon- 
itored using a $20 \mathrm{G}$ radial artery catheter $\left(\mathrm{ABP}_{I A}\right)$ connected via standard low compliant tubing to a disposable pressure transducer (Edwards Lifesciences, Irvine, CA, USA). The non-invasive arterial signal $\left(\mathrm{ABP}_{N I}\right)$ was obtained using a finger cuff adjusted to the size of the index finger according to the guidelines of the manufacturer and connected to the Nexfin monitor (BMEYE Nexfin Monitor [BMEYE, Amsterdam, the Netherlands]). This non-invasive device is based on the principle of Peaz [10] and measures the diameter using an inflatable finger cuff and a built-in photoelectric plethysmograph. From this signal, blood pressure in the brachial artery is reconstructed [11]. Nexfin also calculates beat-to-beat cardiac output $\left(\mathrm{CO}_{N I}\right)$ and stroke volume index $\left(\mathrm{SVI}_{N I}\right)$ using an algorithm called CO-TREK. The area under the systolic portion of the arterial pressure curve is divided by the aortic input impedance [12] and the value of this aortic input impedance is determined by a three-element Windkessel mode, using the influence of the patients age, height, weight and sex on aortic mechanical properties [13]. $\mathrm{CO}_{I A}$ and $\mathrm{SVI}_{I A}$ were determined offline using the stored intra-arterial blood pressure signal derived from the radial artery catheter and the CO-TREK algorithm. Cardiac output was also monitored by transpulmonary thermodilution using the PiCCO monitor $\left(\mathrm{CO}_{T D}\right)$ and a 5-F thermistor-tipped arterial PiCCO catheter inserted in the femoral artery (Pulsion Medical Systems, Munich, Germany). All signals were recorded simultaneously using a sample rate of 200 $\mathrm{Hz}$ and stored on a hard disk. Dynamic indices were calculated from the recorded $\mathrm{ABP}_{I A}$, as well as from the $\mathrm{ABP}_{N I}$ (including $\mathrm{PPV}_{I A}, \mathrm{PPV}_{N I}, \mathrm{SPV}_{I A}, \mathrm{SPV}_{N I}, \mathrm{SVV}_{I A}$ and $\mathrm{SVV}_{N I}$ ) using the average values of the 4 maximum and the 4 mimimum pulse pressures (PP), systolic pressures (SP) and stroke volumes (SV) during 30 seconds: $\mathrm{PPV}=(\mathrm{PPmax} \mathrm{PPmin}) / \mathrm{PPmean}, \mathrm{SPV}=(\mathrm{SPmax} \mathrm{SPmin}) / \mathrm{SPmean}$, and SVV $=(\mathrm{SV}-$ max SVmin)/SVmean. Calculation was performed using the mathematical computer program Matlab (Matlab R2009b, MathWorks Inc., MA, USA), and were visually inspected for errors afterwards.

\subsubsection{Design}

Recording of the data started directly after arrival on the ICU and included all fluid challenges administered by the attending physician based upon the signs of inadequate tissue perfusion (per protocol: low mean arterial pressure (MAP), low urine production, cold extremities, elevated lactate level or low central venous oxygen saturation). Before every fluid challenge ( $6 \mathrm{ml}$ per kg ideal body weight of a 130/0.4 6\% HES solution, Frensenius Kabi, the Netherlands) $\mathrm{CO}_{T D}$ and $\mathrm{CO}_{N I}$ were determined and subsequently, a passive leg-raising (PLR) test was performed [14]. PLR was performed by transferring the patient from a semirecumbent posture to a supine position with leg elevation of $45^{\circ}$. Immediately after the fluid challenge, a second thermodilution measurement was done. Patients were identified as a responder if stroke volume index according to the PiCCO $\left(\mathrm{SVI}_{T D}\right)$ increased by more than $12 \%$. 


\subsubsection{Statistical analysis}

To determine the accuracy of non-invasively derived predictors of fluid responsiveness using the PLR-test we compared the change in $\mathrm{SVI}_{N I}$ and $\mathrm{SVI}_{I A}$ during the PLR-test with the change in $\mathrm{SVI}_{T D}$ after a fluid challenge. Responders were defined as patients where $\mathrm{SVI}_{T D}$ increased $12 \%$ after a fluid challenge. 30 -second time intervals of $\mathrm{ABP}_{N I}, \mathrm{CO}_{N I}$ and $\mathrm{ABP}_{I A}$ were used for further analysis off-line. Haemodynamic parameters were reported as mean standard deviation (SD). Changes in haemodynamic parameters due to the volume expansion were analysed using t-test or the Wilcoxon Rank test and differences in baseline parameters between responders and non-responders were assessed using paired t-test or Mann-Whitney U test (based on distribution). The correlation between the invasively and non-invasively measured PPV, SPV and SVV was tested using the Spearman rank method. Bland Altman analysis was used to assess agreement (bias and limits of agreement) between the invasive and non-invasive derived dynamic indices [15]. For assessing the trending capability of $\mathrm{CO}_{N I}$, we determined concordance using a four-quadrant plot. Receiver operator characteristic (ROC) curves were constructed for both the dynamic indices and the change in CO during PLR to evaluate the predictive value and compared using the method of Delong et. al. [16]. P-values less than 0.05 were considered statistically significant and statistical analysis was done using SPSS Statistics 19.0 for MAC (SPSS Inc., Chicago, Ill.) and MedCalc (MedCalc Software bvba, Belgium).

\subsection{Results}

We included 20 patients, however one patient did not receive a fluid challenge and was excluded from further analysis. Characteristics of the remaining 19 patients are presented in Table 1. In total, patients received 27 fluid challenges of which 14 resulted in a change in SVI $>12 \%$ (52\%). Due to technical difficulties, we were not able to record the intra-arterial pressure signal in 4 patients. Furthermore, one patient was excluded from analysis because he was ventilated in pressure support mode and one patient was excluded because of the presence of arrhythmias. Results regarding the dynamic indices therefore involve 20 fluid challenges (from 15 patients). Three fluid challenges were not preceded by a PLR-test. Therefore, results regarding the PLR therefore involve 24 fluid challenges (from 16 patients). Baseline haemodynamic parameters were not significantly different between responders or non-responders for MAP, HR, GEDV and CVP ( $\mathrm{p}=0.6,0.6,0.48$ and 0.1, respectively). However, SVI was significantly lower in responders $(\mathrm{p}=0.01$, Table 2 ). 


\subsubsection{Dynamic indices}

A strong correlation was found between the dynamic indices derived from the intraarterial waveform and those from the reconstructed brachial waveform measured by the finger cuff ( $r=0.90,0.96$ and 0.74 for PPV, SPV and SVV, respectively and $\mathrm{p}$ $<0.001$ for all correlations). Mean (absolute) bias and limits of agreement were $0.8 \%$ (from $-3.7 \%$ to $2.1 \%$ ), $-0.1 \%$ (from $-1.3 \%$ to $1.0 \%$ ) and $-0.4 \%$ (from $-4.5 \%$ to $3.7 \%$ ) for PPV, SPV and SVV, respectively.

Table 1: Patients characteristics and baseline haemodynamic and respiratory variables. Values are presented as mean $(S D)$ or number.

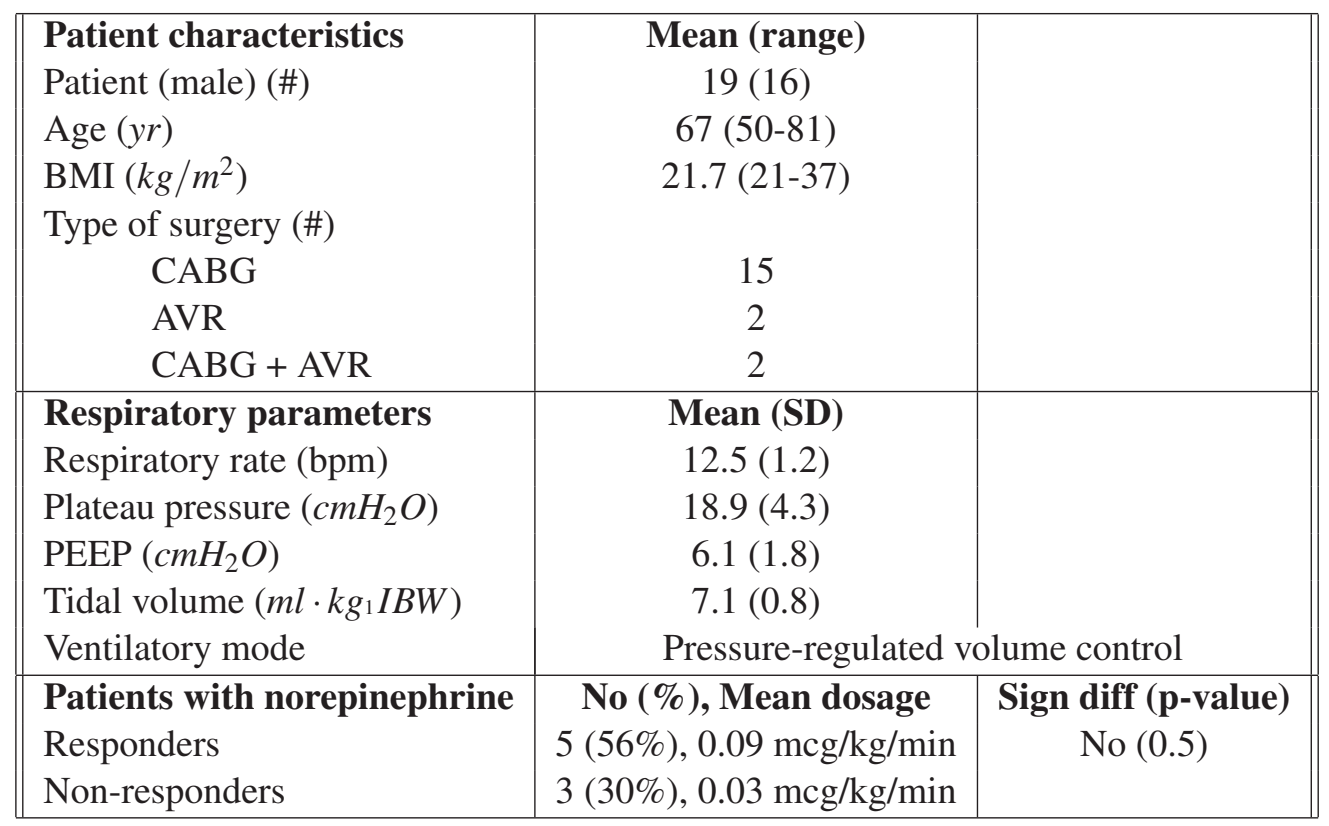

At baseline, PPV and SVV were significantly higher in the fluid responders, SPV was not (see Table 2). The predictive values (area under the curve) of the dynamic indices were $0.83,0.64$ and 0.78 for $\mathrm{PPV}_{I A}, \mathrm{SPV}_{I A}$ and $\mathrm{SVV}_{I A}$ and $0.78,0.55$ and 0.83 for $\mathrm{PPV}_{N I}, \mathrm{SPV}_{N I}$ and $\mathrm{SVV}_{N I}$, respectively (see Figure 1 and Table 3 for more details). Table 3 also shows there was no significant difference in the predictive value of the intra-arterial derived parameters and the parameters calculated non-invasively using a finger cuff. 
Table 2: haemodynamic changes during study protocol. Data are expressed as mean and standard deviation. $*=p<0.05, * *=p<0.01$ (non-responders compared to responders and value after fluid challenge $(F C)$ compared to baseline). $H R=$ heart rate, $C V P=$ central venous pressure, $M A P=$ mean arterial pressure, $S V I=$ stroke volume index, $C O=$ cardiac output, $G E D V=$ global end-diastolic volume, $P P V=$ pulse pressure variation, $S P V=$ systolic pressure variation, $S V V=$ stroke volume variation

\begin{tabular}{|c|c|c|c|c|}
\hline & $\begin{array}{l}\text { Baseline } \\
\mathrm{N}=\mathbf{2 4}\end{array}$ & $\begin{array}{l}\text { PLR } \\
\mathrm{N}=24\end{array}$ & $\begin{array}{l}\text { 5min after } \mathrm{FC} \\
\mathrm{N}=\mathbf{2 4}\end{array}$ & $\begin{array}{l}\text { 30min after } \mathrm{FC} \\
\mathrm{N}=10\end{array}$ \\
\hline $\begin{array}{l}\text { HR }(b / \mathrm{min}) \\
\text { Resp } \\
\text { Non-resp }\end{array}$ & $\begin{array}{l}73(\mathrm{SD} 20) \\
67(\mathrm{SD} 11)\end{array}$ & $\begin{array}{l}73 \text { (SD 19) } \\
68 \text { (SD 10) }\end{array}$ & $\begin{array}{l}73 \text { (SD 19) } \\
68 \text { (SD 10) }\end{array}$ & $\begin{array}{l}75 \text { (SD 23) } \\
75 \text { (SD 13) }\end{array}$ \\
\hline $\begin{array}{l}\text { CVP }(\mathrm{mmHg}) \\
\text { Resp } \\
\text { Non-resp }\end{array}$ & $\begin{array}{l}9.8(\mathrm{SD} 3.2) \\
12.1(\mathrm{SD} 2.7)\end{array}$ & $\begin{array}{l}12.3(\mathrm{SD} 3.6) \\
12.3(\mathrm{SD} 3.7)\end{array}$ & $\begin{array}{l}11.0(\mathrm{SD} 3.5) \\
12.2(\mathrm{SD} 4.4)\end{array}$ & $\begin{array}{l}10.1(\text { SD 5.0) } \\
14.1(\text { SD 3.0) }\end{array}$ \\
\hline $\begin{array}{l}\text { MAP }_{I A}(\mathrm{mmHg}) \\
\text { Resp } \\
\text { Non-resp }\end{array}$ & $\begin{array}{l}70(\mathrm{SD} 7) \\
68(\mathrm{SD} 8)\end{array}$ & $\begin{array}{l}85(\mathrm{SD} 12)^{* * *} \\
80(\mathrm{SD} 9)^{* *}\end{array}$ & $\begin{array}{l}76(\mathrm{SD} 9)^{*} \\
74(\mathrm{SD} 8)\end{array}$ & $\begin{array}{l}88(\mathrm{SD} 11)^{*} \\
76(\mathrm{SD} 8)\end{array}$ \\
\hline $\begin{array}{l}\mathbf{S V I}_{N I}\left(\mathbf{L} / \mathbf{m}^{2}\right) \\
\operatorname{Resp} \\
\text { Non-resp }\end{array}$ & $\begin{array}{l}33.6(\mathrm{SD} 5.0) \\
39.7(\mathrm{SD} 4.9)^{* *}\end{array}$ & $\begin{array}{l}36.1(\text { SD 5.9) } \\
42.2(\text { SD 3.8) }\end{array}$ & $\begin{array}{l}36.9 \text { (SD 5.0) } \\
41.4(\text { SD 5.0) }\end{array}$ & $\begin{array}{l}35.1(\text { SD } 8.1) \\
35.5(\text { SD } 6.0)\end{array}$ \\
\hline $\begin{array}{l}\mathbf{S V I} \mathbf{I}_{T D}\left(\mathbf{L} / \mathbf{m}^{2}\right) \\
\text { Resp } \\
\text { Non-resp }\end{array}$ & $\begin{array}{l}33.4(\mathrm{SD} 8.6) \\
43.2(\mathrm{SD} 5.9)^{* *}\end{array}$ & $\begin{array}{l}36.3 \text { (SD 10.2) } \\
43.3 \text { (SD 6.9) }\end{array}$ & $\begin{array}{l}41.9(\mathrm{SD} 8.7)^{*} \\
44.9(\mathrm{SD} 6.2)\end{array}$ & $\begin{array}{l}38.2(\text { SD 7.0) } \\
41.9(\text { SD 2.9) }\end{array}$ \\
\hline $\begin{array}{l}\mathrm{CO}_{N I}(\mathrm{~L} / \mathrm{min}) \\
\text { Resp } \\
\text { Non-resp }\end{array}$ & $\begin{array}{l}5.0(\mathrm{SD} 1.2) \\
5.3(\mathrm{SD} 1.0)\end{array}$ & $\begin{array}{l}5.4(\mathrm{SD} 1.2) \\
5.7(\mathrm{SD} 1.1)\end{array}$ & $\begin{array}{l}5.6(\mathrm{SD} 1.4) \\
5.6(\mathrm{SD} 1.0)\end{array}$ & $\begin{array}{l}5.3(\mathrm{SD} 1.7) \\
5.7(\mathrm{SD} 1.0)\end{array}$ \\
\hline $\begin{array}{l}\text { GEDVI }_{T D}\left(\mathbf{m l} / \mathbf{m}^{2}\right) \\
\text { Resp } \\
\text { Non-resp }\end{array}$ & $\begin{array}{l}642 \text { (SD 105) } \\
698 \text { (SD 110) }\end{array}$ & & $\begin{array}{l}725 \text { (SD 121) } \\
733 \text { (SD 94) }\end{array}$ & $\begin{array}{l}676 \text { (SD 80) } \\
775 \text { (SD 199) }\end{array}$ \\
\hline $\begin{array}{l}\operatorname{PPV}_{N I}(\%) \\
\text { Resp } \\
\text { Non-resp }\end{array}$ & $\begin{array}{l}12.4(\mathrm{SD} 6.4) \\
7.7(\mathrm{SD} 3.0)^{*}\end{array}$ & & $\begin{array}{l}8.6(\mathrm{SD} 4.9) \\
6.0(\mathrm{SD} 2.7)\end{array}$ & $\begin{array}{l}10.8(\text { SD 5.7) } \\
6.5(\text { SD 1.7) }\end{array}$ \\
\hline $\begin{array}{l}\operatorname{SPV}_{N I}(\%) \\
\text { Resp } \\
\text { Non-resp }\end{array}$ & $\begin{array}{l}6.5(\mathrm{SD} 2.8) \\
5.7(\mathrm{SD} 1.5)\end{array}$ & & $\begin{array}{l}4.6(\mathrm{SD} 2.0)^{*} \\
4.8(\mathrm{SD} 1.6)\end{array}$ & $\begin{array}{l}5.4(\mathrm{SD} 2.4) \\
5.3(\mathrm{SD} 1.8)\end{array}$ \\
\hline $\begin{array}{l}\operatorname{SVV}_{N I}(\%) \\
\text { Resp } \\
\text { Non-resp }\end{array}$ & $\begin{array}{l}10.7(\mathrm{SD} 6.9) \\
6.4(\mathrm{SD} 2.5)^{*}\end{array}$ & & $\begin{array}{l}9.7(\mathrm{SD} 8.4) \\
5.3(\mathrm{SD} 1.8)\end{array}$ & $\begin{array}{l}9.5(\mathrm{SD} 4.0) \\
6.4(\mathrm{SD} 0.7)\end{array}$ \\
\hline
\end{tabular}

\subsubsection{Passive leg-raising test}

Changes in haemodynamic parameters during the study protocol, including the PLR, are depicted in Table 2. PLR increased $\mathrm{CO}_{N I}$ and $\mathrm{CO}_{I A}$ in 21 of 24 and 16 of 18 fluid challenges, respectively and volume infusion increased $\mathrm{CO}_{N I}$ and $\mathrm{CO}_{T D}$ in 23 and 22 


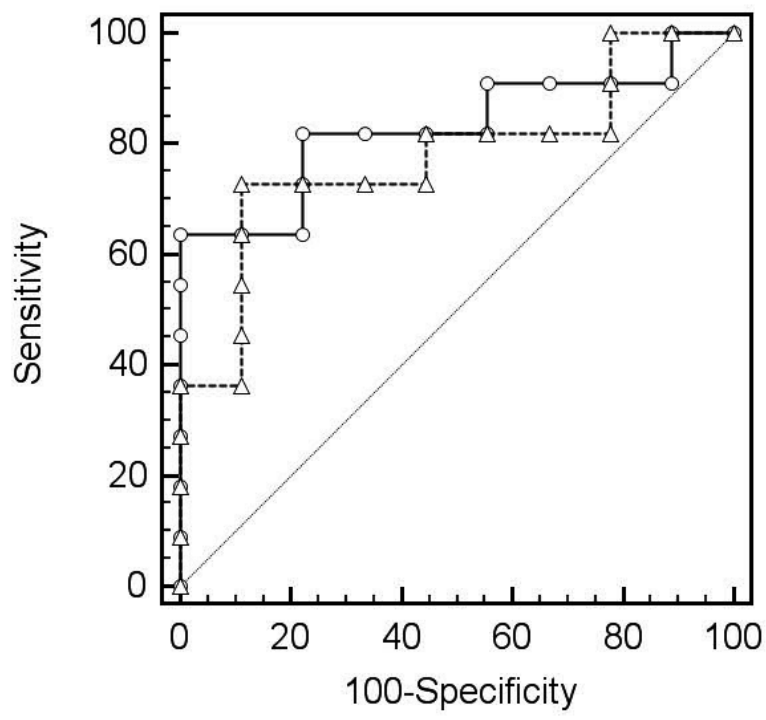

Figure 1: ROC curve analysis for predicting fluid responsiveness using $P P V_{I A}$ (solid line) and $P P V_{N I}$ (dotted line). $P P V=$ pulse pressure variation.

of 24 patients and $\mathrm{CO}_{I A}$ in 16 of 18 patients, respectively. Regarding the direction of change, concordance between changes in $\mathrm{SVI}_{N I}$ after a PLR and changes in $\mathrm{SVI}_{T D}$ after a fluid challenge was $96 \%$ using a 5\% exclusion zone (Figure 2).

The predictive values (area under the curve) of $\mathrm{SVI}_{N I}$ and $\mathrm{SVI}_{I A}$ during a PLRtest were 0.64 (sensitivity $79 \%$ and specificity $60 \%$ ) and 0.68 (sensitivity $91 \%$ and specificity 57\%) respectively (Figure 3). AUCs were not significantly different, see Table 3.

\subsection{Discussion}

We show that fluid responsiveness is acceptably predicted using non-invasively derived dynamic indices in a cardiac surgery population. However, PLR induced changes in SVI measured with Nexfin or intra-arterially using the CO-TREK algorithm were not able to accurately predict fluid responsiveness. In this study, a non-invasive blood pressure signal was obtained in all patients without adverse events. In contrast, in other studies $\mathrm{CO}_{N I}$ was not obtainable in a considerable amount of patients, possibly related to reduced finger arterial flow due to shock and the use of norepinephrine [17-19]. Although in our study $66 \%$ of all patients received norepinephrine, mean 


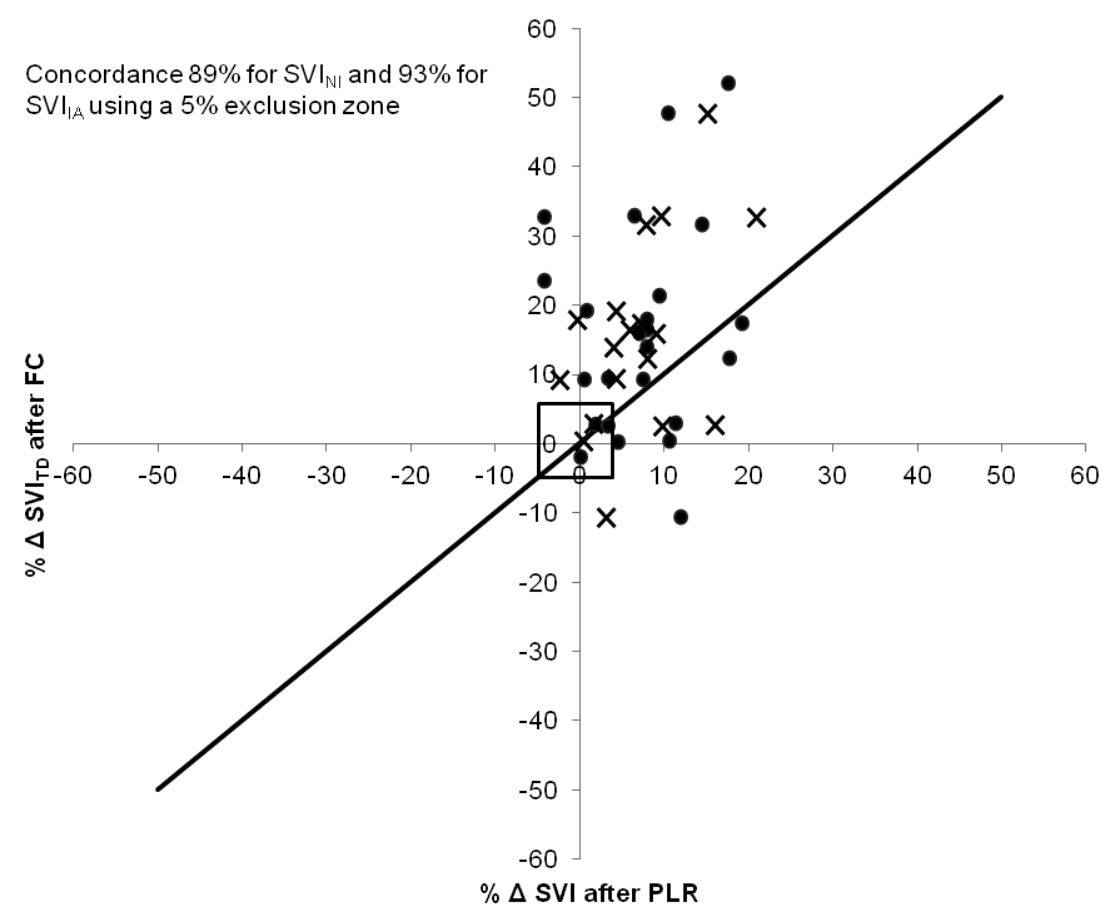

Figure 2: 4-Quadrant concordance analysis using percentage change in $S V I_{N I}$ (dots) and $S V I_{I A}$ (crosses) after PLR plotted against percentage change in $S V I_{T D}$ after $F C . S V I=$ stroke volume index. 
Table 3: results of the ROC curve analysis. $P P V=$ pulse pressure variation, $S P V=$ systolic pressure variation, $S V V=$ stroke volume variation, $C O=$ cardiac output, $S V I=$ stroke volume index.

\begin{tabular}{||l|l|l|l|l|l|l||}
\hline Variable & AUC & Sens & Spec & Treshold & Sign (p) & Sign diff \\
\hline Dynamic indices & & & & & & \\
\hline PPV $_{I A}$ & 0.83 & 65 & 100 & $9.4 \%$ & $<0.01$ & No \\
PPV $_{N I}$ & 0.78 & 73 & 89 & $9.4 \%$ & 0.01 & $(\mathrm{p}=0.42)$ \\
\hline $\mathrm{SPV}_{I A}$ & 0.64 & 55 & 89 & $6.5 \%$ & 0.30 & No \\
SPV $_{N I}$ & 0.55 & 55 & 67 & $6.5 \%$ & 0.70 & $(\mathrm{p}=0.12)$ \\
\hline SVV $_{I A}$ & 0.78 & 55 & 100 & $8.5 \%$ & 0.01 & No \\
SVV $_{N I}$ & 0.83 & 91 & 78 & $8.0 \%$ & $<0.01$ & $(\mathrm{p}=0.60)$ \\
\hline Passive leg raising & & & & & & \\
\hline $\mathrm{CO}_{I A}$ & 0.70 & 64 & 86 & $6.6 \%$ & 0.17 & No \\
$\mathrm{CO}_{N I}$ & 0.56 & 71 & 50 & $6.1 \%$ & 0.60 & $(\mathrm{p}=0.30)$ \\
\hline $\mathrm{SVI}_{I A}$ & 0.68 & 91 & 57 & $3.0 \%$ & 0.26 & No \\
$\mathrm{SVI}_{N I}$ & 0.64 & 79 & 60 & $4.5 \%$ & 0.26 & $(\mathrm{p}=0.66)$ \\
\hline
\end{tabular}

dosage was low compared to a previous studies performed in critically ill patients [18-20]. Fifty-two percent of patients were classified as responders in concordance with previous literature [1]. We used a cut-off point of $12 \%$ as it was determined that this is the smallest relevant change that can be reliably measured using three thermodilution bolus injections [21]. To our knowledge, this is the first study determining the capability of Nexfin of predicting fluid responsiveness using a PLR and subsequent fluid loading. Although concordance of the direction of SVI change between Nexfin and thermodilution after a fluid challenge was high (in line with earlier studies [22]), area under the curve showed limited discriminative value for $\mathrm{SVI}_{N I}$ and $\mathrm{SVI}_{I A}$. This indicates that inaccuracies of SVI measurement are not caused by the non-invasive nature of the measurement. Considering the above, the inability of the CO-TREK algorithm to predict fluid responsiveness using PLR may be caused by the fact that the precision of thermodilution together with the precision of COTREK, which is currently unknown, is too wide to achieve a high AUC with a cut-off point of $12 \%$. Clinical studies are necessary to determine CO-TREK precision. A good agreement was found between the dynamic indices derived non-invasively and invasively, which confirmed our results from a previous study [9]. Furthermore, the present study also shows that non-invasively derived dynamic indices predict fluid responsiveness as good as the invasively derived ones. This eliminates the necessity of invasive haemodynamic monitoring. These results are in agreement with other studies that compared the prediction of non-invasively derived PPV (using Finapres 


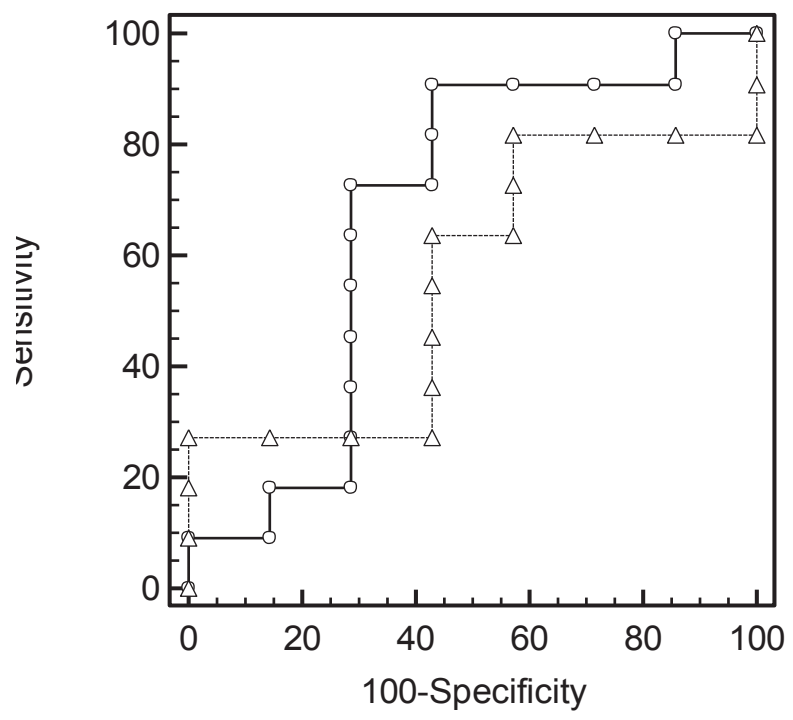

Figure 3: ROC curve analysis for predicting fluid responsiveness using SVI-monitoring using PiCCO $\left(S V I_{I A}\right.$, solid line $)$ and Nexfin $\left(S V I_{N I}\right.$, dotted line $) . S V I=$ stroke volume index

monitor or Infinity CNAP SmartPod) $[23,24]$ and SVV (by oesophageal Doppler) [21] during surgery and in critically ill patients using Nexfin [17]. We found a remarkable difference between the predictive value of SPV compared to PPV and SVV. In previous studies determining the accuracy of finger arterial blood pressure, systolic blood pressure measurement was less accurate than diastolic and mean arterial pressure measurement $[7,25]$. This finding may be caused by the change in the arterial waveform from central arteries to the periphery. This causes a pressure gradient along the arterial tree resulting in pulse wave amplification [26]. Possibly, this phenomenon explains the moderate predictive value of $\mathrm{SPV}_{N I}$ measured at the site of the digital arteries. Some limitations of our study should be noted. First, we used PiCCO as our reference method. Although PiCCO has been shown to be interchangeable with Swan-Ganz thermodilution cardiac output monitoring, it has several limitations, especially during cardiac surgery or episodes of haemodynamic instability [27, 28]. This inaccuracy, caused by the fact that this technique is sensitive to differences in injection technique, respiratory cycle and blood temperature [26], results in a precision error of $12 \%$. This makes the thermodilution technique possibly less reliable as a reference technique under circumstances where small changes in cardiac output occur, as during the PLR. Secondly, we investigated only a small subgroup of patients after cardiac surgery. A recent study investigating the adequacy of Nexfin for tracking the effects of a fluid challenge in septic patients also showed a low re- 
liability. These results were possibly caused by finger hypoperfusion due to sepsis and high dosage of norepinephrine [17]. Whether the results of our study are also applicable to other clinical settings such as trauma care or perioperative care remains to be investigated. Potentially, improved sensitivity of Nexfin could be observed in patients with larger cardiac output changes after fluid loading such as severe hypovolemia or septic shock. Future studies should evaluate the accuracy of Nexfin in these patient categories. This study was conducted in sedated and mechanically ventilated patients. An important patient category where fluid optimisation currently remains a problem are those who are awake and have spontaneous breathing activity. Due to its fast and non-invasive nature, Nexfin could prove to be a valuable tool in haemodynamic optimisation in this clinical setting where invasive monitoring is not possible. However, this remains to be investigated in future studies.

\subsection{Conclusion}

In conclusion, this study showed that Nexfin is a safe and feasible technique for tracking increases in cardiac output in cardiac surgery patients. Although Nexfin is not able to predict fluid responsiveness using passive leg-raising, Nexfin identified responders with a high accuracy using dynamic indices not significantly different from invasively obtained dynamic indices.

\section{References}

[1] F. Michard and J. L. Teboul, "Predicting fluid responsiveness in icu patients: a critical analysis of the evidence," Chest, vol. 121, no. 6, pp. 2000-8, 2002.

[2] S. G. Sakka, M. Klein, K. Reinhart, and A. Meier-Hellmann, "Prognostic value of extravascular lung water in critically ill patients," Chest, vol. 122, no. 6, pp. 2080-6, 2002.

[3] D. Osman, C. Ridel, P. Ray, X. Monnet, N. Anguel, C. Richard, and J. L. Teboul, "Cardiac filling pressures are not appropriate to predict hemodynamic response to volume challenge," Crit Care Med, vol. 35, no. 1, pp. 64-8, 2007.

[4] P. E. Marik, R. Cavallazzi, T. Vasu, and A. Hirani, "Dynamic changes in arterial waveform derived variables and fluid responsiveness in mechanically ventilated patients: a systematic review of the literature," Crit Care Med, vol. 37, no. 9, pp. 2642-7, 2009.

[5] B. Lansdorp, J. Lemson, M. J. van Putten, A. de Keijzer, J. G. van der Hoeven, and P. Pickkers, "Dynamic indices do not predict volume responsiveness 
in routine clinical practice," British journal of anaesthesia, vol. 108, no. 3, pp. 395-401, 2012.

[6] F. Cavallaro, C. Sandroni, C. Marano, G. La Torre, A. Mannocci, C. De Waure, G. Bello, R. Maviglia, and M. Antonelli, "Diagnostic accuracy of passive leg raising for prediction of fluid responsiveness in adults: systematic review and meta-analysis of clinical studies," Intensive care medicine, vol. 36, no. 9, pp. 1475-83, 2010.

[7] J. R. Martina, B. E. Westerhof, J. van Goudoever, E. M. de Beaumont, J. Truijen, Y. S. Kim, R. V. Immink, D. A. Jobsis, M. W. Hollmann, J. R. Lahpor, B. A. de Mol, and J. J. van Lieshout, "Noninvasive continuous arterial blood pressure monitoring with nexfin(r)," Anesthesiology, vol. 116, no. 5, pp. 1092-103, 2012.

[8] O. Broch, J. Renner, M. Gruenewald, P. Meybohm, J. Schottler, A. Caliebe, M. Steinfath, M. Malbrain, and B. Bein, "A comparison of the nexfin(r) and transcardiopulmonary thermodilution to estimate cardiac output during coronary artery surgery," Anaesthesia, vol. 67, no. 4, pp. 377-83, 2012.

[9] B. Lansdorp, D. Ouweneel, A. de Keijzer, J. G. van der Hoeven, J. Lemson, and P. Pickkers, "Non-invasive measurement of pulse pressure variation and systolic pressure variation using a finger cuff corresponds with intra-arterial measurement," British journal of anaesthesia, vol. 107, no. 4, pp. 540-5, 2011.

[10] J. Penaz, A. Voigt, and W. Teichmann, "[contribution to the continuous indirect blood pressure measurement]," Z Gesamte Inn Med, vol. 31, no. 24, pp. 1030-3, 1976.

[11] P. Gizdulich, A. Prentza, and K. H. Wesseling, "Models of brachial to finger pulse wave distortion and pressure decrement," Cardiovascular research, vol. 33, no. 3, pp. 698-705, 1997.

[12] K. H. Wesseling, N. smith, W. Nichols, H. Weber, B. de Wit, and J. E. Beneken, Beat-to-beat cardiac output from the arterial pressure pulse contour, ser. Boerhaave series for postgraduate medical education. Leiden: Leiden University Press, 1974, pp. 148-64.

[13] N. Westerhof, J.-W. Lankhaar, and B. E. Westerhof, "The arterial windkessel," Medical, biological engineering, computing, vol. 47, no. 2, pp. 131-41, 2009.

[14] X. Monnet, M. Rienzo, D. Osman, N. Anguel, C. Richard, M. R. Pinsky, and J. L. Teboul, "Passive leg raising predicts fluid responsiveness in the critically ill," Crit Care Med, vol. 34, no. 5, pp. 1402-7, 2006. 
[15] J. M. Bland and D. G. Altman, "Statistical methods for assessing agreement between two methods of clinical measurement," Lancet, vol. 1, no. 8476, pp. 307-10, 1986.

[16] E. R. DeLong, D. M. DeLong, and D. L. Clarke-Pearson, "Comparing the areas under two or more correlated receiver operating characteristic curves: a nonparametric approach," Biometrics, vol. 44, no. 3, pp. 837-45, 1988.

[17] X. Monnet, M. Dres, A. Ferre, G. Le Teuff, M. Jozwiak, A. Bleibtreu, M. C. Le Deley, D. Chemla, C. Richard, and J. L. Teboul, "Prediction of fluid responsiveness by a continuous non-invasive assessment of arterial pressure in critically ill patients: comparison with four other dynamic indices," British journal of anaesthesia, vol. 109, no. 3, pp. 330-8, 2012.

[18] X. Monnet, F. Picard, E. Lidzborski, M. Mesnil, J. Duranteau, C. Richard, and J. L. Teboul, "The estimation of cardiac output by the nexfin device is of poor reliability for tracking the effects of a fluid challenge," Critical care, vol. 16, no. 5, p. R212, 2012.

[19] M. O. Fischer, R. Avram, I. Carjaliu, M. Massetti, J. L. Gerard, J. L. Hanouz, and J. L. Fellahi, "Non-invasive continuous arterial pressure and cardiac index monitoring with nexfin after cardiac surgery," British journal of anaesthesia, vol. 109, no. 4, pp. 514-21, 2012.

[20] D. W. Eeftinck Schattenkerk, J. J. van Lieshout, A. H. van den Meiracker, K. R. Wesseling, S. Blanc, W. Wieling, G. A. van Montfrans, J. J. Settels, K. H. Wesseling, and B. E. Westerhof, "Nexfin noninvasive continuous blood pressure validated against riva-rocci/korotkoff," Am J Hypertens, vol. 22, no. 4, pp. 378 83, 2009.

[21] X. Monnet, R. Persichini, M. Ktari, M. Jozwiak, C. Richard, and J.-L. Teboul, "Precision of the transpulmonary thermodilution measurements," Critical care (London, England), vol. 15, no. 4, p. R204, 2011.

[22] M. Helmi, R. B. de Wilde, J. R. Jansen, B. F. Geerts, M. I. Versteegh, P. C. van den Berg, D. Gommers, and A. B. Groeneveld, "Comparing hemodynamic effects with three different measurement devices, of two methods of external leg compression versus passive leg raising in patients after cardiac surgery," Journal of clinical monitoring and computing, 2012.

[23] H. Solus-Biguenet, M. Fleyfel, B. Tavernier, E. Kipnis, J. Onimus, E. Robin, G. Lebuffe, C. Decoene, F. R. Pruvot, and B. Vallet, "Non-invasive prediction of fluid responsiveness during major hepatic surgery," Br J Anaesth, vol. 97, no. 6, pp. 808-16, 2006. 
[24] M. Biais, L. Stecken, L. Ottolenghi, S. Roullet, A. Quinart, F. Masson, and F. Sztark, "The ability of pulse pressure variations obtained with cnap device to predict fluid responsiveness in the operating room," Anesthesia and analgesia, vol. 113, no. 3, pp. 523-8, 2011.

[25] B. P. Imholz, W. Wieling, G. A. van Montfrans, and K. H. Wesseling, "Fifteen years experience with finger arterial pressure monitoring: assessment of the technology," Cardiovasc Res, vol. 38, no. 3, pp. 605-16, 1998.

[26] M. Botero, D. Kirby, E. B. Lobato, E. D. Staples, and N. Gravenstein, "Measurement of cardiac output before and after cardiopulmonary bypass: Comparison among aortic transit-time ultrasound, thermodilution, and noninvasive partial co2 rebreathing," Journal of cardiothoracic and vascular anesthesia, vol. 18, no. 5, pp. 563-72, 2004.

[27] P. J. Peyton and S. W. Chong, "Minimally invasive measurement of cardiac output during surgery and critical care: a meta-analysis of accuracy and precision," Anesthesiology, vol. 113, no. 5, pp. 1220-35, 2010.

[28] J. Pugsley and A. B. Lerner, "Cardiac output monitoring: is there a gold standard and how do the newer technologies compare?" Seminars in cardiothoracic and vascular anesthesia, vol. 14, no. 4, pp. 274-82, 2010. 


\section{Summary, general discussion and future perspectives}





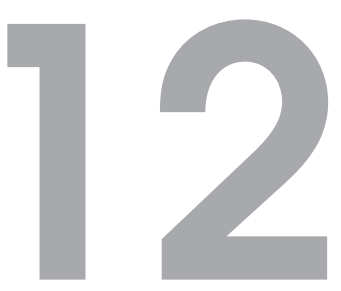

Summary, general discussion and future perspectives 
Predicting whether a patient with acute circulatory failure will benefit from fluid administration by a significant increase in stroke volume is a daily clinical challenge in the intensive care unit. For this reason, predicting fluid responsiveness in critically ill patients has become one of the major topics of anesthesia and intensive care research during the last decades. Static markers of volume status like blood pressure, central venous pressure, pulmonary capillary wedge pressure or right ventricular end-diastolic volume have been studied for many years. Nevertheless, these preload markers do not accurately predict fluid responsiveness [1] because a given value of preload can correspond to either a large or a negligible response of cardiac output to fluid administration, depending upon the slope of the Frank-Starling curve.

This thesis therefore focused on the use of dynamic indices for predicting fluid responsiveness in mechanically ventilated patients, based on the swings in stroke volume and arterial blood pressure due to heart-lung interactions [2]. Our research included an assessment of the clinical usefulness in adults and neonates and a more fundamental approach to the origin and basic physiology behind the dynamic indices, which is summarized in a mathematical simulation model. Furthermore, we explored potential non-invasive techniques to assess fluid responsiveness.

\section{Summary}

\section{Part I - Dynamic indices and fluid responsiveness}

In chapter 2 we assessed the value of the dynamic parameters to predict fluid responsiveness in patients on controlled mechanical ventilation, patients on assisted mechanical ventilation and spontaneously breathing patients. We conclude that after diagnosing inadequate organ perfusion, the first step is to determine the patient's heart rhythm. In the presence of an irregular heart rhythm, a passive leg raising (PLR) test with cardiac output monitoring is the most valid option because the use of dynamic indices and the use of echocardiography to assess the collapsibility of the inferior or superior caval vein have not been validated in this situation. Also in patients on assisted mechanical ventilation, the only method validated in the literature is the PLR test. A small fluid challenge with a cardiac output measurement can be performed if PLR is not possible. In spontaneously breathing patients, the use of dynamic indices might be an option. However, although it has been shown that specificity still may be high, sensitivity is low and stroke volume or pulse pressure variation cannot be advised to predict fluid responsiveness in these patient and again the PLR test or a traditional fluid challenge is preferable. If the patient is on controlled mechanical ventilation with a tidal volume $\geq 8 \mathrm{ml} / \mathrm{kg}$ and has a regular heart rhythm, we advise the use of pulse pressure of stroke volume variation. If pulse pressure- or stroke volume variation is $>12 \%$, we advise fluid administration if clinical or biochemical signs of tissue hypoperfusion are present. 
In chapter 3 we put this theory to the test and evaluated the clinical usefulness of dynamic indices in 47 fluid challenges and quantified to what extent the predictive value was attenuated when the above-described requirements are not met. We confirmed that the predictive value of dynamic indices is high in the group of patients ventilated with high tidal volume (TV> $7 \mathrm{ml} / \mathrm{kg}$ ) without cardiac arrhythmias and with the indices calculated breath-by-breath. We also showed that the use of a lower tidal volume, the presence of cardiac arrhythmias and calculating the indices not on a breath-by-breath basis, reduced the area under the receiver operator curve to almost half. On the other hand, correction of the dynamic indices for tidal volume (by dividing the dynamic indices by tidal volume) increases its reliability.

Arterial pressure variations can also be observed in mechanically ventilated neonates. However, the frequency at which pulse pressure varies does not always correspond with the respiratory frequency. Because of this observed mismatch, we questioned whether the arterial pressure swings were indeed related to the ventilation and if they could be used for the prediction of fluid responsiveness. In chapter 4 we showed that although the observed pulse pressure variation in neonates on synchronized intermittent mandatory ventilation or high frequency ventilation is indeed related to cardiopulmonary interaction, it is unlikely that it will reliably predict fluid responsiveness. This is because the ratio of the heart rate to the respiratory rate is too low to adequately reflect respiratory changes within the arterial blood pressure signal.

\section{Part II - Intra-thoracic pressure distribution during mechanical ventila- tion in health and disease}

The limited use of the dynamic preload indicators in daily clinical practice is likely explained by a lack of understanding of the exact physiology. For this reason, we aimed to improve our knowledge of the underlying physiology of heart-lung interaction. In chapter 5 we determined the extent to which the airway pressure induced by mechanical ventilation is distributed within the thorax and interacts with the circulation and showed how this is influenced by tidal volume and chest compliance. We demonstrated that the ratio of airway pressure that is distributed to the pleura (about $2 / 3 \mathrm{rd}$ ) is almost double the percentage distributed to the pericard and caval vein (about $1 / 3$ rd), that tidal volume in a clinically relevant range does not influences these percentages, and that a lower chest wall compliance resulted in a larger portion of airway pressure that is transmitted to the intrathoracic pressures. Based on our results, we adapted the published threshold values for the dynamic predictors of fluid responsiveness according to the applied tidal volume and present chest wall compliance. Additionally, we showed that in patients with higher values of dynamic indices (likely to be hypovolemic), the transmural pressure of the right atrium decreases with increasing tidal volumes, while in patients with lower values of dynamic 
indices (likely to be normovolemic) this transmural pressure increases. This finding indicates that in hypovolemic patients the decreased preload is the main determinant for the lower output of the right heart, while in non-responders, the increased afterload is the main determinant.

Besides the chest wall compliance, also the lung compliance influences the extent to which the airway pressure is transmitted within the thorax. However, in chapter 6 we showed in a pediatric animal model that it is not the transpulmonary pressure (related to lung compliance), but the pleural pressure (determined by the tidal volume divided by chest wall compliance) that determines the value of the dynamic indices. Another parameter that influences heart-lung interaction, and thereby the value of the dynamic preload indicators, is the intra-abdominal pressure. While it is known that the predictive value of dynamic indices is much lower during abdominal surgery, we explained these prior findings in chapter 7 by showing that by opening the abdominal compartment, the dynamic indices are approximately halved. This is most probably caused by diminished heart-lung interactions due to the increased thoracic wall compliance due to a decrease in abdominal pressure following the opening of the abdomen.

The large number of physiological parameters involved in the mechanisms behind the dynamic indices makes them difficult to interpret at the bedside for clinical use. A better understanding of the complex relationships between tidal volume, chest wall compliance and dynamic indices in relation to fluid responsiveness could expand their usability. For this reason, we constructed a physiologically based mathematical model of the interaction between the respiratory and cardiovascular systems, incorporating dynamic indices and fluid responsiveness in chapter 8. Our model is based on existing models of the cardiovascular system and the respiratory system during mechanical ventilation. The model of the cardiovascular system is expanded by including non-linear cardiac elastance to improve simulation of the Frank-Starling mechanism, which, as explained before, plays an important role in the relation between volume status and dynamic indices. The described model allows for the simulation of these complex relationships and may predict the effect of volume infusion in specific patients in the future.

\section{Part III - Non-invasive techniques to assess fluid responsiveness}

In the ICU, the blood pressure waveform, which is needed for the calculation of the dynamic indices, is typically measured with an intra-arterial catheter. It is also useful to estimate dynamic indices in situations where intra-arterial monitoring is not available. The ability to monitor pulse pressure and stroke volume variation noninvasively increases the clinical usefulness in patients without an arterial catheter, for example for patients undergoing surgery or in acute situations in the emergency room. Non-invasive blood pressure monitors, like the Finapres NOVA (Finapres Medical 
Systems) and the Nexfin monitor (Edwards), are able to continuously monitor arterial blood pressure non-invasively using an inflatable finger cuff with a build-in photo-electric plethysmograph. In chapter 10 we show that in patients receiving controlled mechanical ventilation, pulse pressure variation and systolic pressure variation can be measured reliably using an inflatable finger cuff. However, the use of dynamic indices is restricted to a limited number of patients, and other predictors of fluid responsiveness like the PLR-test or a traditional fluid challenge, still require cardiac output monitoring. We therefore also evaluated the accuracy of a cardiac output calculation based on the arterial waveform measured with a finger cuff. In a comparison between the traditional transpulmonary thermodilution method and the cardiac output calculated by pulse contour analysis of the blood pressure waveform measured with a finger cuff, we showed that although these methods are not interchangeable, the non-invasive method closely followed changes in cardiac output chapter 9.

The ability to monitor the dynamic indices and to track changes in cardiac output using a finger cuff, suggests that this non-invasive method could be used to predict fluid responsiveness in patients without an intra-arterial pressure catheter. In an observational study, described in chapter 11, we confirmed this hypothesis for the prediction of fluid responsiveness using dynamic indices. However, although the dynamic indices derived from the finger cuff showed a good predictive value, and the concordance of the direction of the change in stroke volume index between the invasive and non-invasive cardiac output measurement was high, non-invasively derived changes in cardiac output during the PLR-test could not predict fluid responsiveness reliably. This is probably caused by the fact that the precision of the invasive technique (thermodilution) together with the precision of non-invasive technique to determine cardiac output, which is currently unknown, is too wide to achieve a high AUC with a cut-off point of $12 \%$.

\section{General discussion and future perspectives}

The shift from the traditional static indices to the dynamic indices for the prediction of fluid responsiveness resulted in several parameters that can be used as a control variable to guide fluid administration. Of these parameters, stroke volume variation is supported with the highest level of evidence [3,4]. For this reason, implementation of early goal-directed hemodynamic therapy based on dynamic indices has been able to show a reduction of complications and length of stay after cardiac surgery [5-7]. The quantitative and objective character of the dynamic indices also facilitates automated management of fluid resuscitation [8]. In simulation and in vivo studies, it was demonstrated that a closed loop administration system based on dynamic indices is an effective volumetric resuscitator $[9,10]$. These closed loop fluid administration techniques are actually precursors of mathematical, physiology-based 
models. While the closed loop fluid administration is rule- or algorithm based, our biophysical model described in chapter 8 incorporates the cardiopulmonary system and its interactions in relation to dynamic indices and fluid responsiveness and can be adapted to individual patients and thereby serve as a bedside clinical decision support system. Furthermore, mathematical models are successfully used for educational simulations teaching complex physiology $[11,12]$.

However, due to several restrictions, the dynamic indices are still not widely applied. This is because less than half of the patients during anesthesia meet the criteria for the usage of dynamic indices [13] and as we showed in chapter 3, the predictive value of dynamic indices is substantially reduced in patients that do not meet these criteria. This has also been recognized by others $[4,14]$. So in order for the dynamic indices to become more widely applicable, some of these limitations have to be overcome.

\section{Limitations of dynamic preload indicators}

One of the major limitations was the reliance on invasive monitoring techniques while the majority of patients requiring fluid therapy are not appropriate for invasive monitoring [15]. Fortunately, as we have shown in chapter 10 and 11, and also suggested by others [16], pulse pressure variation and systolic pressure variation can be measured reliably using an inflatable finger cuff. These advances in non-invasive monitoring of blood pressure and cardiac output make the dynamic indices feasible for a wider range of patients and also make (closed loop) therapy based on dynamic indices applicable in a variety of clinical care settings [17, 18].

Besides the systolic pressure, pulse pressure and stroke volume variation addressed in this thesis, also other non-invasively derived indices that provide a beat-tobeat surrogate of stroke volume have been investigated for their ability to predict fluid responsiveness over the recent years. One of them is the plethysmographic variability index, which is based on the swings in the pulse oximetry plethysmographic waveform that is widely used in intensive care units. However, although proof of concept studies in stable hemodynamic conditions reported encouraging results [19, 20], recent studies in ICU patients report poor accuracy [21, 22]. Echocardiography based parameters, like aortic blood flow velocity $[23,24]$ and respiratory variation in inferior or superior vena cava diameter $[25,26]$ also have the ability to predict fluid responsiveness. Although all these studies still require confirmation, and are known to be more labor-intensive and operator dependent, they may be of great interest for the perioperative management of low-risk surgical patients, when there is no need for an invasive monitoring device.

With the ability to track the dynamic indices non-invasively and this limitation has been overcome, the first and most important limitation in the use of dynamic indices is the presence of spontaneous breathing activity. As a result of the sponta- 
neous breathing activity, the frequency and amplitude of the variation in intrathoracic pressure is irregular and cannot be used to predict volume responsiveness [27-30].

The second limitation is the use of low tidal volumes, as is currently the standard of care. The smaller variations in intrathoracic pressure (directly related to tidal volume) are insufficient to trigger significant preload variations, even in patients with preload responsiveness. For this reason, the preferred hemodynamic parameter in patients ventilated with a tidal volume $<8 \mathrm{ml} / \mathrm{kg}$ is still a matter of debate. While the specificity of pulse pressure- or stroke volume variation may be high in ARDS patients ventilated with a low tidal volume [31], lower predictive values in a mixed group of intensive care patients using tidal volume $<8 \mathrm{ml} / \mathrm{kg}$ were also found [32]. Especially in patients with severe ARDS fluid overload should be prevented because in these patients, excessive fluid overload is deleterious [33-37]. In chapter 2 we advised the PLR-test or a fluid challenge in combination with cardiac output monitoring to guide further fluid administration in case of ARDS.

To overcome the limitation of low tidal volume, is has been suggested that dynamic indices should be indexed to the tidal volume [38]. The principle of indexation of the dynamic indices is based on the concept that ideally, dynamic indices quantify the slope of the Frank-Starling curve. However, the dynamic parameters only quantify the cardiac output component as a result of the changing preload, neglecting the absolute variation in preload (as the slope of the curve is defined as the change in cardiac output divived by the change in preload). In chapter 5 , we determined the exact distribution of intrathoracic pressure as a result of different tidal volumes and provide quantitative data in order to correctly index the dynamic parameters. In parallel, the presence of a high chest wall compliance or open-chest conditions [39, 40] will also result in less pronounced variations in intrathoracic pressure, reducing the interaction with the circulation and the ability of the dynamic indices to predict volume responsiveness. Correction for the value of the patient's chest compliance can also be performed based on the results acquired in chapter 5 .

Some authors also suggested that a decreased lung compliance itself (or total respiratory system compliance $<30 \mathrm{~mL} / \mathrm{cmH} 20$ ) limits the use of dynamic indices [33]. This is based on the assumption that a decrease in lung compliance will increase the airway driving pressure, resulting in sufficient swings in transpulmonary pressure to maintain the predictive value of the dynamic indices [31, 33, 41]. However, in chapter 6 we provided physiological evidence that the value of the dynamic indices is more closely related to the change in pleural pressure (influenced by tidal volume and chest wall compliance) than the transpulmonary pressure. This implies that dynamic indices are unlikely to be useful in cases of ARDS because of the preferred use of low tidal volumes (TV) [42] and the accompanying small variations in pleural pressure. This is in accordance to the clinical findings of others that these variations might not be sufficient to trigger significant preload variations, even in cases of obvious preload 
responsiveness $[32,34,43,44]$.

The presence of increased abdominal pressure may also limit the predictive value of the dynamic indices $[45,46]$. This is confirmed by our findings in chapter 7 , where we found that opening the abdomen decreases pulse pressure variation and stroke volume variation. Therefore, during conditions in which the abdomen is opened, adjustments of cut-off values for dynamic indices for predicting fluid responsiveness are required.

A fifth and sixth limitation is the presence of cardiac arrhythmias and a low heart rate to respiratory rate ratio. In case of cardiac arrhythmias, the ventilation-induced variation of hemodynamic parameters loses its predictive value because the variations are more related to the irregularity of the heart rate, rather than to heart-lung interactions. In the case of a low heart rate to respiratory rate the number of heartbeats per respiratory cycle is too low to reflect the true respiratory variations due to under sampling. This physiological principle was previously found in adults [47], but has a also been related to the cyclic variations in arterial blood pressure in neonates by us in chapter 4. Although the frequency of these variations does not always correspond with the respiratory rate, we demonstrated that the variations are indeed caused by cardiopulmonary interactions and that the mismatch is caused by the low heart rate to respiratory rate ratio. However, despite this relation, we conclude that it is likely that dynamic indices will not be able to reliably predict fluid responsiveness because the occurrence of the low heart rate to respiratory rate ratio impedes the quantitative analysis of the dynamic indices.

Another important limitation related to the cardiac performance is the presence of right ventricular dysfunction. This is based on two principles. First, as right ventricular dysfunction leads to a decreased right ventricular ejection during the inspiratory increase of right ventricular afterload, infused fluid will not increase left ventricular stroke volume [48]. Second, due to the increase in right ventricular volume, the intra-ventricular septum will shift towards the left ventricle, impeding left ventricular output. Doppler tricuspid annular systolic velocity (Sta) can be used to quantify right ventricular function and to detect false-positive values of pulse pressure variation [49].

Finally, the use of vasoconstrictors is suggested to be another limitation. Vasopressive drugs modify vascular tone, reduce the degree of preload dependency and seem to impair the ability of pulse pressure variation to predict fluid responsiveness [50-52]. This might be because arterial pressure surrogates do not reliably reflect variations in stroke volume as knowledge about peripheral vascular resistance is often incomplete. Direct measurement of stroke volume (variation) might be the solution to overcome this limitation. Table 1 summarizes all above-mentioned limitations. 
Table 1: Limitations of dynamic preload indicators. Sta= Doppler tricuspid annular systolic velocity

\begin{tabular}{||l||}
\hline \multicolumn{1}{|c||}{ Limitation of dynamic preload indicators } \\
\hline Spontaneous breathing activity \\
Low tidal volume $(<8 \mathrm{ml} / \mathrm{kg})$ \\
Low chest wall compliance or open chest conditions \\
Increased abdominal pressure \\
Cardiac arrhythmias \\
Low heart rate to respiratory rate $(<3.6)$ \\
Right ventricular dysfunction $(\mathrm{Sta}<0.15 \mathrm{~m} / \mathrm{s})$ \\
High dosage of vasoactive medication \\
\hline
\end{tabular}

\section{Alternative techniques to predict fluid responsiveness}

In the presence of one or more of the above-mentioned limitations, several alternative methods for the prediction of fluid responsiveness are available. The alternative with the highest level of evidence, and thus the most preferred one, is the passive legraising test in combination with cardiac output monitoring [53]. For this purpose, invasive monitoring of cardiac output is preferred, since there is still no consensus in the literature of using non-invasive tracking of cardiac output by pulse contour for the prediction of fluid responsiveness $[54,55]$. This reluctance to use non-invasive methods to determine cardiac output seems to be valid because in chapter 11, we also showed that the Nexfin is not able to predict fluid responsiveness using the passive leg-raising test. On the other hand, measuring the change in end-tidal carbon dioxide seems to be a good alternative, where the change in PP is not $[33,53,56]$. However, for this technique, also mixed results have been found [57] and more evidence is needed before it can be implemented in clinical practice.

In situations where passive leg raising test is not possible, several other methods have been developed that, similar to the dynamic indices, are based on changes in intrathoracic pressure. In patients with low tidal volumes, e.g. due to low lung compliance, the end-expiratory occlusion test may be a reliable predictor [33]. This test consists of interrupting tidal ventilation at end-expiration for a few seconds and provokes an increase in venous return and, in cases of preload responsiveness, an increase in cardiac output. According to the same physiological principle, changes in central venous pressure during the use of incremental levels of PEEP also appear to be a good parameter to predict fluid responsiveness in mechanically ventilated patients [58]. However, in patients with decreased lung compliance, the predictive value will be less since the change in lung volume will be insufficient to expose preload dependency. Furthermore, in mechanically ventilated patients with spontaneous breathing 
activity, paced breathing with or without the use of respiratory resistors to increase changes in intrathoracic pressure and thereby changes in preload might also provide valuable information $[59,60]$. In this category, the square root of the pulse pressure power spectrum has also shown to predict volume responsiveness without the need for sedation to prevent spontaneous breathing activity [61]. Also in spontaneously breathing patients, the changes in central venous pressure and vena cava diameter might be useful to assess preload dependency. This is because a decrease in central venous pressure during inspiration (causing the cardiac function curve as introduced in the introduction of this thesis to shift to the left) indicates that the heart operates on the steep part of the cardiac function curve and might benefit from fluid infusion [28, 62-64]. However, probably the most reliable and feasible alternative besides the passive leg-raising test is the admission of a fluid challenge (small fluid bolus), as this technique is widely applicable and has proven its accuracy repeatedly to guide further resuscitation [65]. As a final alternative, the mean systemic filling pressure might also be helpful in assessing the patients volume status (at least in theory, [66]). The mean systemic filling pressure represents the intravascular volume that stresses the vascular walls that, and together with the unstressed volume, reflects the intravascular volume. Since the mean systemic filling pressure, relative to central venous pressure, is the driving pressure for venous return (and thus cardiac output), measuring mean systemic filling pressure, in combination with the cardiac function curve, can be helpful during hemodynamic management of spontaneously breathing patients $[67,68]$. In order to assess the mean systemic filling pressure at the bedside, several techniques are available [69].

In Chapter 6, we have shown in that the change of the pressure gradient between the mean systemic filling pressure (measured as the pressure in a peripheral vein) and the central venous pressure during intermittent positive airway pressure or prolonged periods of positive airway pressure was related to the value of the dynamic indices. In patients with low values of stroke volume variation this pressure gradient hardly changed and in patients with high values of stroke volume variation (likely to be fluid responders), this pressure gradient dropped to values of $50 \%$ of the initial value. This indicates that non-responders are not experiencing any drop in preload during positive pressure ventilation which is in accordance to our findings in chapter 5 which suggests a dominant contribution of afterload in normovolemic patients and a dominant role of preload in hypovolemic patients in heart-lung interactions during mechanical ventilation. Our results therefore underline the potential clinical value of the mean systemic filling pressure with respect to fluid management on the ICU.

\section{Fluid responsiveness versus the need for fluids}

Before assessing the question of fluid responsiveness in critically ill patients, it is important to determine if a patient actually needs an increase in cardiac output. In- 
creasing stroke volume in patients with adequate organ perfusion serves no useful purpose and might even be harmful. Overzealous fluid resuscitation has been associated with increased complications, increased length of ICU and hospital stay, and increased mortality [70-72]. This has been subscribed to a series of events that occurs when the heart is operating on or near the flat part of the Frank-Starling curve and fluid loading has little effect on the stroke volume. In this case, the beneficial effects of fluid loading are reduced and volume expansion becomes progressively detrimental, as illustrated in Figure 1. The disability of the heart to increase stroke volume will raise cardiac filling pressures that stimulates the release of natriuretic peptides, damaging the endothelial glycocalyx and resulting in an increase in extravascular lung water and interstitial edema due to an higher endothelial permeability [73-75]. Recently it was shown that a high central venous pressure is related to the development of acute kidney injury in patients with sepsis. This may be explained by a decrease in glomerular perfusion pressure.

Figure 1 illustrates that being fluid responsive does not necessarily imply hypovolemia and that a fluid challenge should only be given to patients who are likely to show a significant increase in stroke volume with improved tissue and organ perfusion (see Table 2).

The central assumption for using macro-circulatory parameters for fluid management is that an increase in cardiac output will lead to the actual goal of fluid resuscitation: an improvement in tissue oxygenation, which is only true if oxygen consumption depends on oxygen delivery. Although an increase in cardiac output has been related to improved sublingual microcirculation [76], a recent study showed that in only about half of the patients with an increase in cardiac output $>15 \%$, a significant increase in oxygen consumption occurred [77]. Parallel to this, macro circulatory resuscitation endpoints like arterial and central venous pressure as well as cardiac output should be reconsidered. Instead, only a minimum individual and context-specific mean arterial blood pressure in combination with endpoints of tissue perfusion should be targeted [78]. These recommendations are strengthened by the findings that assessment of microvascular blood flow helps to identify patients eligible for fluid therapy [77, 79, 80]. Recent technological advances like videomicroscopic devices, measuring tissue $\mathrm{PCO}_{2}$ 4and near infrared spectroscopy might also facilitate the monitoring of tissue perfusion [81]. However, although these measurements looks promising, higher level of evidence is needed before these parameters can be used as endpoints for fluid resuscitation.

\section{Conclusion}

Despite an explosion of research concerning the prediction of fluid responsiveness using dynamic indices over the last decade, the clinical application of these techniques is still limited. Most likely, the underlying physiology is still too complex, 


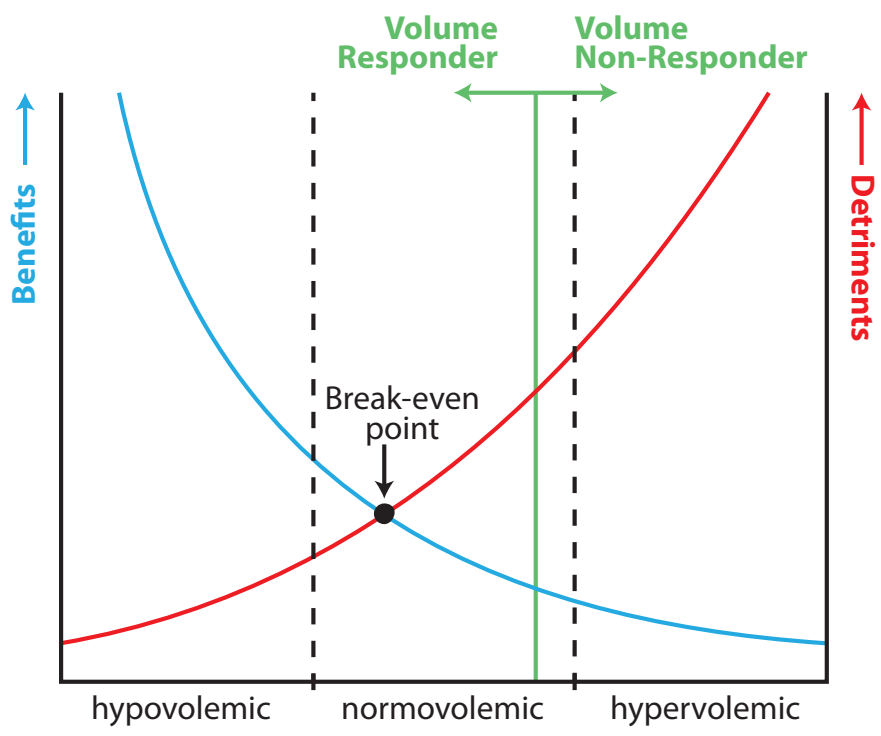

Figure 1: The benefits of fluid administration decrease (left vertical axis) with increasing amounts of intravascular fluid (blue curve, horizontal axis). Due to the inability of the heart to increase stroke volume with increasing preload (Frank-Starling-curve), volume expansion becomes progressively detrimental (red curve, right vertical axis). The figure shows that although the largest part of the normovolemic patients is volume responsive (upper part of the Figure), the detriments may have a more negative influence on patients health status that the benefits will add $(A=$ break even point). For this reason, the risk/benefit ratio should be considered very well in each patient. 
Table 2: clinical indices of tissue perfusion

\begin{tabular}{||l||}
\hline \multicolumn{1}{|c||}{ Clinical indices of tissue and organ perfusion } \\
\hline Urine output \\
Mentation \\
Capillary refill \\
Skin perfusion/mottling \\
Cold extremities \\
Blood lactate \\
Arterial Ph, Be, and $\mathrm{HCO} 3$ \\
Central venous and mixed venous oxygen saturation \\
Mixed venous pCO2 \\
Tissue pCO2 \\
Skeletal muscle tissue oxygenation
\end{tabular}

and corresponding limitations too numerous for routine clinical use. For this reason, future research should focus on other more fundamental physiology closely related to volume status (e.g. the mean systemic filling pressure) or on more endpoint related parameters like measuring mitochondrial oxygen tension which can give insight in the oxygen balance [82]. In any case, in order to become widely applicable, these new parameters should be easy to assess (preferably non- or minimally-invasive) and be relatively insensitive for accompanying conditions like mechanical ventilation or the cardiac rhythm disturbances. These characteristics are essential for alternative parameters to become more successful than the passive leg raising test or the traditional fluid challenge, and will allow application outside the OR or the ICU, as well, for example in the emergency department or in dialysis units.

\section{References}

[1] P. E. Marik and R. Cavallazzi, "Does the central venous pressure predict fluid responsiveness? an updated meta-analysis and a plea for some common sense," Critical care medicine, vol. 41, no. 7, pp. 1774-81, 2013.

[2] F. Michard and J. L. Teboul, "Using heart-lung interactions to assess fluid responsiveness during mechanical ventilation," Crit Care, vol. 4, no. 5, pp. 282-9, 2000.

[3] P. E. Marik, R. Cavallazzi, T. Vasu, and A. Hirani, "Dynamic changes in arterial waveform derived variables and fluid responsiveness in mechanically ventilated 
patients: a systematic review of the literature," Crit Care Med, vol. 37, no. 9, pp. 2642-7, 2009.

[4] X. Monnet and J. L. Teboul, "Assessment of volume responsiveness during mechanical ventilation: recent advances," Critical care, vol. 17, no. 2, p. 217, 2013.

[5] M. S. Goepfert, H. P. Richter, C. Zu Eulenburg, J. Gruetzmacher, E. Rafflenbeul, K. Roeher, A. von Sandersleben, S. Diedrichs, H. Reichenspurner, A. E. Goetz, and D. A. Reuter, "Individually optimized hemodynamic therapy reduces complications and length of stay in the intensive care unit: a prospective, randomized controlled trial," Anesthesiology, vol. 119, no. 4, pp. 824-36, 2013.

[6] S. M. Lobo and N. E. de Oliveira, "Clinical review: What are the best hemodynamic targets for noncardiac surgical patients?" Critical care, vol. 17, no. 2, p. 210, 2013.

[7] S. M. Lobo, C. L. Mendes, E. Rezende, and F. S. Dias, "Optimizing perioperative hemodynamics: what is new?" Current opinion in critical care, vol. 19, no. 4, pp. 346-52, 2013.

[8] J. Rinehart, N. Liu, B. Alexander, and M. Cannesson, "Review article: closedloop systems in anesthesia: is there a potential for closed-loop fluid management and hemodynamic optimization?" Anesthesia and analgesia, vol. 114, no. 1 , pp. 130-43, 2012.

[9] J. Rinehart, B. Alexander, Y. Le Manach, C. Hofer, B. Tavernier, Z. N. Kain, and M. Cannesson, "Evaluation of a novel closed-loop fluid-administration system based on dynamic predictors of fluid responsiveness: an in silico simulation study," Critical care, vol. 15, no. 6, p. R278, 2011.

[10] J. Rinehart, C. Lee, C. Canales, A. Kong, Z. Kain, and M. Cannesson, "Closedloop fluid administration compared to anesthesiologist management for hemodynamic optimization and resuscitation during surgery: an in vivo study," Anesthesia and analgesia, vol. 117, no. 5, pp. 1119-29, 2013.

[11] V. C. Rideout, "Cardiovascular system simulation in biomedical engineering education," IEEE Trans Biomed Eng, vol. 19, no. 2, pp. 101-7, 1972.

[12] H. A. Rossow and J. H. Stern, "Teaching comparative metabolism using a graphic computer model, virtual tissue," Advances in Physiology Education, vol. 35, no. 1, pp. 99-102, 2011. 
[13] S. Maguire, J. Rinehart, S. Vakharia, and M. Cannesson, "Technical communication: respiratory variation in pulse pressure and plethysmographic waveforms: intraoperative applicability in a north american academic center," Anesth Analg, vol. 112, no. 1, pp. 94-6, 2011.

[14] Y. Mahjoub, V. Lejeune, L. Muller, S. Perbet, L. Zieleskewicz, F. Bart, B. Veber, C. Paugam-Burtz, S. Jaber, A. Ayham, E. Zogheib, S. Lasocki, A. VieillardBaron, H. Quintard, O. Joannes-Boyau, G. Plantefeve, P. Montravers, S. Duperret, M. Lakhdari, N. Ammenouche, E. Lorne, M. Slama, and H. Dupont, "Evaluation of pulse pressure variation validity criteria in critically ill patients: a prospective observational multicentre point-prevalence study," British journal of anaesthesia, 2013.

[15] H. Wilms, A. Mittal, M. D. Haydock, M. van den Heever, M. Devaud, and J. A. Windsor, "A systematic review of goal directed fluid therapy: Rating of evidence for goals and monitoring methods," Journal of critical care, 2013.

[16] X. Monnet, M. Dres, A. Ferre, G. Le Teuff, M. Jozwiak, A. Bleibtreu, M. C. Le Deley, D. Chemla, C. Richard, and J. L. Teboul, "Prediction of fluid responsiveness by a continuous non-invasive assessment of arterial pressure in critically ill patients: comparison with four other dynamic indices," British journal of anaesthesia, vol. 109, no. 3, pp. 330-8, 2012.

[17] J. Truijen, J. J. van Lieshout, W. A. Wesselink, and B. E. Westerhof, "Noninvasive continuous hemodynamic monitoring," Journal of clinical monitoring and computing, vol. 26, no. 4, pp. 267-78, 2012.

[18] M. Geisen, A. Rhodes, and M. Cecconi, "Less-invasive approaches to perioperative haemodynamic optimization," Current opinion in critical care, vol. 18, no. 4, pp. 377-84, 2012.

[19] P. Forget, F. Lois, and M. de Kock, "Goal-directed fluid management based on the pulse oximeter-derived pleth variability index reduces lactate levels and improves fluid management," Anesthesia and analgesia, vol. 111, no. 4, pp. 910-4, 2010.

[20] C. Sandroni, F. Cavallaro, C. Marano, C. Falcone, P. De Santis, and M. Antonelli, "Accuracy of plethysmographic indices as predictors of fluid responsiveness in mechanically ventilated adults: a systematic review and meta-analysis," Intensive care medicine, vol. 38, no. 9, pp. 1429-37, 2012.

[21] M. O. Fischer, A. Pelissier, D. Bohadana, J. L. Gerard, J. L. Hanouz, and J. L. Fellahi, "Prediction of responsiveness to an intravenous fluid challenge in patients after cardiac surgery with cardiopulmonary bypass: a comparison be- 
tween arterial pulse pressure variation and digital plethysmographic variability index," Journal of cardiothoracic and vascular anesthesia, vol. 27, no. 6, pp. 1087-93, 2013.

[22] X. Monnet, L. Guerin, M. Jozwiak, A. Bataille, F. Julien, C. Richard, and J. L. Teboul, "Pleth variability index is a weak predictor of fluid responsiveness in patients receiving norepinephrine," British journal of anaesthesia, vol. 110, no. 2, pp. 207-13, 2013.

[23] X. Monnet, M. Rienzo, D. Osman, N. Anguel, C. Richard, M. R. Pinsky, and J. L. Teboul, "Esophageal doppler monitoring predicts fluid responsiveness in critically ill ventilated patients," Intensive care medicine, vol. 31, no. 9, pp. 1195-201, 2005.

[24] M. Feissel, F. Michard, I. Mangin, O. Ruyer, J. P. Faller, and J. L. Teboul, "Respiratory changes in aortic blood velocity as an indicator of fluid responsiveness in ventilated patients with septic shock," Chest, vol. 119, no. 3, pp. 867-73, 2001.

[25] M. Feissel, F. Michard, J. P. Faller, and J. L. Teboul, "The respiratory variation in inferior vena cava diameter as a guide to fluid therapy," Intensive Care Med, vol. 30, no. 9, pp. 1834-7, 2004.

[26] C. Barbier, Y. Loubieres, C. Schmit, J. Hayon, J. L. Ricome, F. Jardin, and A. Vieillard-Baron, "Respiratory changes in inferior vena cava diameter are helpful in predicting fluid responsiveness in ventilated septic patients," Intensive Care Med, vol. 30, no. 9, pp. 1740-6, 2004.

[27] S. Delerme, S. Castro, Y. Freund, P. Nazeyrollas, M. O. Josse, B. MadonnaPy, E. Rouff, B. Riou, and P. Ray, "Relation between pulse oximetry plethysmographic waveform amplitude induced by passive leg raising and cardiac index in spontaneously breathing subjects," The American journal of emergency medicine, vol. 28, no. 4, pp. 505-10, 2010.

[28] L. Muller, X. Bobbia, M. Toumi, G. Louart, N. Molinari, B. Ragonnet, H. Quintard, M. Leone, L. Zoric, and J. Y. Lefrant, "Respiratory variations of inferior vena cava diameter to predict fluid responsiveness in spontaneously breathing patients with acute circulatory failure: need for a cautious use," Critical care, vol. 16, no. 5, p. R188, 2012.

[29] L. Bodson and A. Vieillard-Baron, "Respiratory variation in inferior vena cava diameter: surrogate of central venous pressure or parameter of fluid responsiveness? let the physiology reply," Critical care, vol. 16, no. 6, p. 181, 2012. 
[30] S. T. Vistisen, P. Juhl-Olsen, C. A. Frederiksen, and H. Kirkegaard, "Variations in the pre-ejection period induced by deep breathing do not predict the hemodynamic response to early haemorrhage in healthy volunteers," Journal of clinical monitoring and computing, 2013.

[31] C. C. Huang, J. Y. Fu, H. C. Hu, K. C. Kao, N. H. Chen, M. J. Hsieh, and Y. H. Tsai, "Prediction of fluid responsiveness in acute respiratory distress syndrome patients ventilated with low tidal volume and high positive end-expiratory pressure," Crit Care Med, vol. 36, no. 10, pp. 2810-6, 2008.

[32] D. De Backer, S. Heenen, M. Piagnerelli, M. Koch, and J. L. Vincent, "Pulse pressure variations to predict fluid responsiveness: influence of tidal volume," Intensive Care Med, vol. 31, no. 4, pp. 517-23, 2005.

[33] X. Monnet, A. Bleibtreu, A. Ferre, M. Dres, R. Gharbi, C. Richard, and J. L. Teboul, "Passive leg-raising and end-expiratory occlusion tests perform better than pulse pressure variation in patients with low respiratory system compliance," Critical care medicine, vol. 40, no. 1, pp. 152-7, 2012.

[34] K. Lakhal, S. Ehrmann, and T. Boulain, "Pulse pressure variation: does lung compliance really matter?" Critical care medicine, vol. 40, no. 5, pp. 1691; author reply 1691-2, 2012.

[35] L. Muller, G. Louart, P. J. Bousquet, D. Candela, L. Zoric, J. E. de La Coussaye, S. Jaber, and J. Y. Lefrant, "The influence of the airway driving pressure on pulsed pressure variation as a predictor of fluid responsiveness," Intensive care medicine, vol. 36, no. 3, pp. 496-503, 2010.

[36] J. Mesquida, H. K. Kim, and M. R. Pinsky, "Effect of tidal volume, intrathoracic pressure, and cardiac contractility on variations in pulse pressure, stroke volume, and intrathoracic blood volume," Intensive care medicine, vol. 37, no. 10, pp. 1672-9, 2011.

[37] D. De Backer and S. Scolletta, "Why do pulse pressure variations fail to predict the response to fluids in acute respiratory distress syndrome patients ventilated with low tidal volume?" Critical care, vol. 15, no. 2, p. 150, 2011.

[38] S. T. Vistisen, J. Koefoed-Nielsen, and A. Larsson, "Should dynamic parameters for prediction of fluid responsiveness be indexed to the tidal volume?" Acta Anaesthesiol Scand, 2009.

[39] E. E. de Waal, S. Rex, C. L. Kruitwagen, C. J. Kalkman, and W. F. Buhre, "Dynamic preload indicators fail to predict fluid responsiveness in open-chest conditions," Crit Care Med, vol. 37, no. 2, pp. 510-5, 2009. 
[40] P. A. Wyffels, P. Sergeant, and P. F. Wouters, "The value of pulse pressure and stroke volume variation as predictors of fluid responsiveness during open chest surgery," Anaesthesia, vol. 65, no. 7, pp. 704-9, 2010.

[41] J. Mallat, M. Lemyze, and D. Thevenin, "Ability of respiratory pulse pressure variation to predict fluid responsiveness in ards: still an unanswered question?" Critical care, vol. 15, no. 3, p. 432; author reply 432, 2011.

[42] ARDS-network, "Ventilation with lower tidal volumes as compared with traditional tidal volumes for acute lung injury and the acute respiratory distress syndrome. the acute respiratory distress syndrome network," $N$ Engl J Med, vol. 342, no. 18, pp. 1301-8, 2000.

[43] K. Lakhal, S. Ehrmann, D. Benzekri-Lefevre, I. Runge, A. Legras, P. F. Dequin, E. Mercier, M. Wolff, B. Regnier, and T. Boulain, "Respiratory pulse pressure variation fails to predict fluid responsiveness in acute respiratory distress syndrome," Critical care, vol. 15, no. 2, p. R85, 2011.

[44] J. Y. Lefrant and D. De Backer, "Can we use pulse pressure variations to predict fluid responsiveness in patients with ards?" Intensive Care Med, vol. 35, no. 6, pp. 966-8, 2009.

[45] D. Jacques, K. Bendjelid, S. Duperret, J. Colling, V. Piriou, and J. P. Viale, "Pulse pressure variation and stroke volume variation during increased intraabdominal pressure: an experimental study," Critical care, vol. 15, no. 1, p. R33, 2011.

[46] B. Tavernier and E. Robin, "Assessment of fluid responsiveness during increased intra-abdominal pressure: keep the indices, but change the thresholds," Critical care, vol. 15, no. 2, p. 134, 2011.

[47] D. De Backer, F. S. Taccone, R. Holsten, F. Ibrahimi, and J. L. Vincent, "Influence of respiratory rate on stroke volume variation in mechanically ventilated patients," Anesthesiology, vol. 110, no. 5, pp. 1092-7, 2009.

[48] A. Vieillard-Baron, K. Chergui, A. Rabiller, O. Peyrouset, B. Page, A. Beauchet, and F. Jardin, "Superior vena caval collapsibility as a gauge of volume status in ventilated septic patients," Intensive Care Med, vol. 30, no. 9, pp. 1734-9, 2004.

[49] J. J. Maas, B. F. Geerts, P. C. van den Berg, M. R. Pinsky, and J. R. Jansen, "Assessment of venous return curve and mean systemic filling pressure in postoperative cardiac surgery patients," Crit Care Med, vol. 37, no. 3, pp. 912-8, 2009. 
[50] M. Biais, V. Cottenceau, L. Petit, F. Masson, J. F. Cochard, and F. Sztark, "Impact of norepinephrine on the relationship between pleth variability index and pulse pressure variations in icu adult patients," Critical care, vol. 15, no. 4, p. R168, 2011.

[51] X. Monnet, J. Jabot, J. Maizel, C. Richard, and J.-L. Teboul, "Norepinephrine increases cardiac preload and reduces preload dependency assessed by passive leg raising in septic shock patients," Critical care medicine, vol. 39, no. 4, pp. 689-94, 2011.

[52] J. P. Bouchacourt, J. A. Riva, and J. C. Grignola, "The increase of vasomotor tone avoids the ability of the dynamic preload indicators to estimate fluid responsiveness," BMC anesthesiology, vol. 13, no. 1, p. 41, 2013.

[53] F. Cavallaro, C. Sandroni, C. Marano, G. La Torre, A. Mannocci, C. De Waure, G. Bello, R. Maviglia, and M. Antonelli, "Diagnostic accuracy of passive leg raising for prediction of fluid responsiveness in adults: systematic review and meta-analysis of clinical studies," Intensive care medicine, vol. 36, no. 9, pp. $1475-83,2010$.

[54] M. O. Fischer, J. Coucoravas, J. Truong, L. Zhu, J. L. Gerard, J. L. Hanouz, and J. L. Fellahi, "Assessment of changes in cardiac index and fluid responsiveness: a comparison of nexfin and transpulmonary thermodilution," Acta anaesthesiologica Scandinavica, vol. 57, no. 6, pp. 704-12, 2013.

[55] X. Monnet, F. Picard, E. Lidzborski, M. Mesnil, J. Duranteau, C. Richard, and J. L. Teboul, "The estimation of cardiac output by the nexfin device is of poor reliability for tracking the effects of a fluid challenge," Critical care, vol. 16, no. 5, p. R212, 2012.

[56] X. Monnet, A. Bataille, E. Magalhaes, J. Barrois, M. Le Corre, C. Gosset, L. Guerin, C. Richard, and J. L. Teboul, "End-tidal carbon dioxide is better than arterial pressure for predicting volume responsiveness by the passive leg raising test," Intensive care medicine, vol. 39, no. 1, pp. 93-100, 2013.

[57] P. G. Guinot, J. Godart, B. de Broca, E. Bernard, E. Lorne, and H. Dupont, "End-expiratory occlusion manoeuvre does not accurately predict fluid responsiveness in the operating theatre," British journal of anaesthesia, 2014.

[58] B. F. Geerts, L. P. Aarts, A. B. Groeneveld, and J. R. Jansen, "Predicting cardiac output responses to passive leg raising by a peep-induced increase in central venous pressure, in cardiac surgery patients," British journal of anaesthesia, vol. 107, no. 2, pp. 150-6, 2011. 
[59] E. Zollei, V. Bertalan, A. Nemeth, P. Csabi, I. Laszlo, J. Kaszaki, and L. Rudas, "Non-invasive detection of hypovolemia or fluid responsiveness in spontaneously breathing subjects," BMC anesthesiology, vol. 13, no. 1, p. 40, 2013.

[60] P. Grassi, L. Lo Nigro, K. Battaglia, M. Barone, F. Testa, and G. Berlot, "Pulse pressure variation as a predictor of fluid responsiveness in mechanically ventilated patients with spontaneous breathing activity: a pragmatic observational study," HSR proceedings in intensive care and cardiovascular anesthesia, vol. 5, no. 2, pp. 98-109, 2013.

[61] C. H. Lee, J. Y. Wang, Y. K. Wu, H. W. Chiu, C. C. Lan, H. Chang, and C. Y. Chen, "Pulse pressure power spectrum predicts volume responsiveness in shock patients without sedation," Shock, vol. 33, no. 5, pp. 454-9, 2010.

[62] S. Magder, G. Georgiadis, and T. Cheong, "Respiratory variations in right atrial pressure predict the response to fluid challenge," J Crit Care, vol. 7, no. 2, pp. 76-85, 1992.

[63] S. Magder, "Bench-to-bedside review: An approach to hemodynamic monitoring - guyton at the bedside," Critical care, vol. 16, no. 5, p. 236, 2012.

[64] M. J. Lanspa, C. K. Grissom, E. L. Hirshberg, J. P. Jones, and S. M. Brown, "Applying dynamic parameters to predict hemodynamic response to volume expansion in spontaneously breathing patients with septic shock," Shock, vol. 39, no. 2, pp. 155-60, 2013.

[65] L. Muller, M. Toumi, P. J. Bousquet, B. Riu-Poulenc, G. Louart, D. Candela, L. Zoric, C. Suehs, J. E. de La Coussaye, N. Molinari, and J. Y. Lefrant, "An increase in aortic blood flow after an infusion of $100 \mathrm{ml}$ colloid over 1 minute can predict fluid responsiveness: the mini-fluid challenge study," Anesthesiology, vol. 115, no. 3, pp. 541-7, 2011.

[66] W. G. Parkin and M. S. Leaning, “Therapeutic control of the circulation," Journal of clinical monitoring and computing, vol. 22, no. 6, pp. 391-400, 2008.

[67] M. Cecconi, H. D. Aya, M. Geisen, C. Ebm, N. Fletcher, R. M. Grounds, and A. Rhodes, "Changes in the mean systemic filling pressure during a fluid challenge in postsurgical intensive care patients," Intensive care medicine, vol. 39, no. 7, pp. 1299-305, 2013.

[68] J. J. Maas, M. R. Pinsky, L. P. Aarts, and J. R. Jansen, "Bedside assessment of total systemic vascular compliance, stressed volume, and cardiac function curves in intensive care unit patients," Anesthesia and analgesia, vol. 115, no. 4, pp. 880-7, 2012. 
[69] J. J. Maas, M. R. Pinsky, B. F. Geerts, R. B. de Wilde, and J. R. Jansen, "Estimation of mean systemic filling pressure in postoperative cardiac surgery patients with three methods," Intensive care medicine, vol. 38, no. 9, pp. 1452-60, 2012.

[70] A. L. Rosenberg, R. E. Dechert, P. K. Park, and R. H. Bartlett, "Review of a large clinical series: association of cumulative fluid balance on outcome in acute lung injury: a retrospective review of the ardsnet tidal volume study cohort," $J$ Intensive Care Med, vol. 24, no. 1, pp. 35-46, 2009.

[71] J. H. Boyd, J. Forbes, T. A. Nakada, K. R. Walley, and J. A. Russell, "Fluid resuscitation in septic shock: a positive fluid balance and elevated central venous pressure are associated with increased mortality," Critical care medicine, vol. 39, no. 2, pp. 259-65, 2011.

[72] S. Brandt, T. Regueira, H. Bracht, F. Porta, S. Djafarzadeh, J. Takala, J. Gorrasi, E. Borotto, V. Krejci, L. B. Hiltebrand, L. E. Bruegger, G. Beldi, L. Wilkens, P. M. Lepper, U. Kessler, and S. M. Jakob, "Effect of fluid resuscitation on mortality and organ function in experimental sepsis models," Crit Care, vol. 13, no. 6, p. R186, 2009.

[73] P. E. Marik and J. Lemson, "Fluid responsiveness: an evolution of our understanding," British journal of anaesthesia, 2014.

[74] D. Bruegger, L. Schwartz, D. Chappell, M. Jacob, M. Rehm, M. Vogeser, F. Christ, B. Reichart, and B. F. Becker, "Release of atrial natriuretic peptide precedes shedding of the endothelial glycocalyx equally in patients undergoing on- and off-pump coronary artery bypass surgery," Basic research in cardiology, vol. 106, no. 6, pp. 1111-21, 2011.

[75] Z. Zhang, Y. Xue, X. Xu, and H. Ni, "Prognostic value of b-type natriuretic peptide (bnp) and its potential role in guiding fluid therapy in critically ill septic patients," Scandinavian journal of trauma, resuscitation and emergency medicine, vol. 20, p. 86, 2012.

[76] J. Pottecher, S. Deruddre, J. L. Teboul, J. F. Georger, C. Laplace, D. Benhamou, E. Vicaut, and J. Duranteau, "Both passive leg raising and intravascular volume expansion improve sublingual microcirculatory perfusion in severe sepsis and septic shock patients," Intensive care medicine, vol. 36, no. 11, pp. 1867-74, 2010.

[77] X. Monnet, F. Julien, N. Ait-Hamou, M. Lequoy, C. Gosset, M. Jozwiak, R. Persichini, N. Anguel, C. Richard, and J. L. Teboul, "Lactate and venoarterial carbon dioxide difference/arterial-venous oxygen difference ratio, but not central 
venous oxygen saturation, predict increase in oxygen consumption in fluid responders," Critical care medicine, vol. 41, no. 6, pp. 1412-20, 2013.

[78] M. W. Dunser, J. Takala, A. Brunauer, and J. Bakker, "Re-thinking resuscitation: leaving blood pressure cosmetics behind and moving forward to permissive hypotension and a tissue perfusion-based approach," Critical care, vol. 17, no. 5, p. 326, 2013.

[79] J. Boldt and C. Ince, "The impact of fluid therapy on microcirculation and tissue oxygenation in hypovolemic patients: a review," Intensive care medicine, vol. 36, no. 8, pp. 1299-308, 2010.

[80] A. Pranskunas, M. Koopmans, P. M. Koetsier, V. Pilvinis, and E. C. Boerma, "Microcirculatory blood flow as a tool to select icu patients eligible for fluid therapy," Intensive care medicine, vol. 39, no. 4, pp. 612-9, 2013.

[81] D. De Backer, K. Donadello, and D. O. Cortes, "Monitoring the microcirculation," Journal of clinical monitoring and computing, vol. 26, no. 5, pp. 361-6, 2012.

[82] E. G. Mik, "Special article: measuring mitochondrial oxygen tension: from basic principles to application in humans," Anesthesia and analgesia, vol. 117, no. 4, pp. 834-46, 2013. 


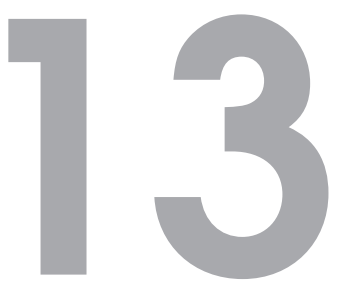

Samenvatting in het Nederlands 


\section{Samenvatting}

Dit proefschrift beschrijft ons onderzoek naar de optimalisatie van de bloedsomloop (circulatie) bij kritiek zieke patiënten op de intensive care. Het belangrijkste doel van de bloedsomloop is het aanvoeren van zuurstof naar de verschillende weefsels en organen in het menselijk lichaam. Bij patiënten op de intensive care afdeling is de circulatie vaak verstoord, waardoor een adequate zuurstof-voorziening niet gewaarborgd is. Een verminderd zuurstof aanbod aan de weefsels kan leiden tot een langere ziekteduur en zelfs het overlijden van een patiënt. Het beoordelen van de circulatie van de patiënt is dan ook een dagelijks terugkerend fenomeen op de intensive care. Tegelijkertijd is dit een van de moeilijkste handelingen, omdat een falende circulatie veroorzaakt kan worden door een (combinatie van) verminderde hartfunctie, verminderde vaatfunctie of een verlaagde hoeveelheid circulerend bloed.

Het toedienen van extra vocht aan de bloedsomloop wordt vaak gezien als de eerste stap in de behandeling van een inadequate circulatie. In het ideale geval zal deze toename van het circulerend volume leiden tot een verhoging van het hartminuutvolume, de hoeveelheid bloed die het hart per minuut rondpompt. Het blijkt echter dat bij 50\% van de patiënten deze vochttoediening niet leidt tot een verhoging van het hartminuutvolume en dus ook niet tot een verhoging van het zuurstofaanbod aan de weefsels. In deze patiënten, de zogenaamde non-responders, kan overvulling leiden tot oedeemvorming, hartfalen, verlengde ziekenhuisopname en zelfs overlijden.

Het is dan ook belangrijk om in de praktijk parameters te hebben die de respons op een volume bolus (volume responsiviteit) voorspellen, ofwel, onderscheid kunnen maken tussen volume responders en non-responders, om zo het hartminuutvolume te optimaliseren en de kans op overvulling te minimaliseren. Het voorspellen van de volume responsiviteit is de afgelopen decennia dan ook een belangrijk onderwerp geweest in het anesthesie en intensive care onderzoek. Hierbij is gebleken dat symptomen als urineproductie en temperatuur van de huid, maar ook statische indicatoren voor de volume-status zoals arteriële bloeddruk of centraal veneuze druk, onbetrouwbaar zijn in het voorspellen van de effecten van een volume toediening.

In dit proefschrift hebben we ons gericht op het gebruik van dynamische indices als parameters voor het voorspellen van volume responsiviteit. Deze dynamische indices zijn variaties in de bloeddruk of slagvolume van het hart die ontstaan als gevolg van drukvariaties in de thorax (intrathoracale drukvariaties) tijdens mechanische beademing. Een verhoging van de intrathoracale druk bij inademing zal leiden tot een verlaging van de vulling (of vullingsdruk ofwel preload) en een verhoging van de uitstroomweerstand (of afterload) van het rechterventrikel (rechter hartkamer). Dit leidt bij preload-gevoelige patiënten tot een daling van het slagvolume van het rechterventrikel tijdens de (mechanische) inademing, wat vervolgens zorgt voor een daling van het slagvolume van het linkerventrikel tijdens de uitademing. Deze dy- 
namische indices, waar de pulsdruk variatie (PPV) een voorbeeld van is, geeft dus aan of er bij de patiënt sprake is van preload-reserve (en het hart opereert op het steile deel van de hartfunctie curve, wat aangeeft dat een patiënt baat heeft bij extra vulling), of dat de vulling van het hart al verzadigd is (en het hart opereert op het vlakke deel van de hartfunctie curve, wat aangeeft dat een volume bolus mogelijk schadelijk is voor de patiënt).

Een inleiding in dit onderwerp wordt gegeven in Hoofdstuk 1. De overige hoofdstukken, opgedeeld in 3 delen, beschrijven ons onderzoek. Dit omvat een assessment van de klinische toepasbaarheid van de dynamische indices in volwassenen en neonaten (pasgeborene) en een meer fundamentele zoektocht naar de oorsprong van de dynamische indices, samengevat in een mathematisch simulatie model met als doel het vergroten van de betrouwbaarheid van dynamische indices met betrekking tot het voorspellen van volume responsiviteit. Tevens hebben we naar mogelijkheden gekeken om volume responsiviteit vast te stellen op basis van niet-invasieve metingen.

\section{Deel I - Dynamische indices en volume responsiviteit}

In Hoofdstuk 2 hebben we de waarde van de dynamische indices als voorspeller van volume responsiviteit beoordeeld bij patiënten met gecontroleerde mechanische beademing, geassisteerde mechanische beademing en spontane ademhaling. Wij concluderen dat de eerste stap na het vaststellen van een inadequate circulatie bij een patiënt, het beoordelen van het hartritme is. Bij een irregulair hartritme is het toepassen van dynamische indices namelijk niet gevalideerd en wordt een 'passiveleg-raising-test' (PLR-test) aangeraden in combinatie met een hartminuutvolumemeting. Hierbij worden de benen van de patiënt omhoog getilt zodat er bloed uit de benen naar het hart stroomt en zo een kunstmatige en omkeerbare volume bolus gesimuleerd wordt. De PLR-test is ook de beste keus bij patiënten die geassisteerd beademd worden of spontaan ademen. Wanneer een PLR-test niet mogelijk is, kan deze vervangen worden door de snelle toediening van een kleine hoeveelheid vocht, ook in combinatie met een hartminuutvolume-meting. Bij patiënten aan de gecontroleerde beademing met een teugvolume groter dan $8 \mathrm{ml} / \mathrm{kg}$, een regulair hartritme en een pulsdruk variatie groter dan $12 \%$ adviseren wij een volume bolus.

In Hoofdstuk 3 hebben we deze theorie geëvalueert in 47 volume bolussen bij IC patiënten. Daarbij hebben we de gevolgen onderzocht met betrekking tot de voorspellende waarde van de dynamische indices wanneer er niet aan de voorwaarden van regulaire hartritme en mechanische beademing voldaan wordt. Hieruit bleek inderdaad dat de voorspellende waarde hoog is bij de patiëntenpopulatie die beademd wordt met teugvolumes groter dan $7 \mathrm{ml} / \mathrm{kg}$, een regulair hartritme hebben en waarbij de dynamische indices ademteug voor ademteug berekend worden. Echter, het bleek ook dat deze voorspellende waarde werd gereduceerd tot 0.5 bij patiënten die 
beademd werden met teugvolumes kleiner dan $7 \mathrm{ml} / \mathrm{kg}$ en waarbij de dynamische indices berekend werden op basis van 30 seconden zonder dat mogelijk aanwezige onregelmatigheden in het hartritme werden gefilterd.

In neonaten die mechanisch beademd worden zijn deze dynamische indices ook waar te nemen. De frequentie waarmee de pulsdruk variëert komt echter vaak niet overeen met de ademhalings frequentie. Hierdoor is het onduidelijk of de ademhaling wel de oorzaak is van de waargenomen pulsdruk variaties, en of deze potentieel bruikbaar zijn bij het voorspellen van de gevolgen van volume therapie volgens dezelfde theorie als bij volwassen IC patiënten. In Hoofdstuk $\mathbf{4}$ hebben wij aangetoond dat ondanks dat deze frequentie niet altijd overeen komt, de variaties in pulsdruk inderdaad veroorzaakt worden door de beademing. Echter, door het zeer lage aantal hartslagen per ademteug, die ook de oorzaak blijkt van de mismatch tussen de frequentie van de variatie van de pulsdruk en die van de ademhaling, is het onwaarschijnlijk dat de dynamische indices ook bij neonaten kunnen worden gebruikt voor het voorspellen van volume responsiviteit.

\section{Deel II - distributie van de luchtwegdruk tijdens mechanische beademing}

De grote hoeveelheid beperkingen en de daarmee gepaarde beperkte toepasbaarheid van dynamische indices in de dagelijkse praktijk, wordt naar alle waarschijnlijkheid onder andere veroorzaakt door een gebrek aan inzicht in de exacte fysiologie. Om deze reden hebben we in het tweede deel van dit proefschrift gekeken naar de achterliggende principes van de hart-long interactie om het begrip hiervan te vergroten. In Hoofdstuk 5 hebben we bepaald in welke mate de verhoging van de luchtwegdruk als gevolg van mechanische beademing wordt doorgegeven aan de verschillende compartimenten in de thorax (borstkas), en hoe dit de circulatie beïnvloedt. Ook hebben we het effect van teugvolume en elasticiteit van de thoraxwand hierop onderzocht. Door het meten van de druk rondom de longen (pleurale ruimte) en de druk in het hartzakje bij patiënten na een open-hart operatie hebben wij aangetoond dat ongeveer $2 / 3$ de van de luchtwegdruk wordt doorgegeven aan de pleuraholte. Bij het hartzakje is dit ongeveer $1 / 3 d$. Deze percentages werden niet beïnvloed door het variëren van het teugvolume, maar werden wel verhoogd door een afname van de elasticiteit van de thoraxwand. Aan de hand van deze resultaten hebben wij een voorstel gedaan om de huidige drempelwaardes van de dynamische indices aan te passen aan het toegepaste teugvolume en de aanwezige elasticiteit van de thorax.

Naast elasticiteit van de thoraxwand, is ook de elasticiteit van de long bepalend voor de mate waarin de luchtwegdruk wordt doorgeven aan de rest van de thorax. In Hoofdstuk 6 hebben wij in een pediatrisch dierenmodel laten zien dat met afgenomen elasticiteit van de long (dit werd bereikt door de surfactant van de longen van de lammetjes uit te spoelen door middel van lavage), de distributie van de luchtwegdruk naar de pleurale ruimte inderdaad wordt verminderd. Daarentegen hebben wij in deze 
studie ook aangetoond dat niet de transpulmonale druk (gerelateerd aan de long elasticiteit), maar voornamelijk de pleurale druk (bepaald door het teugvolume gedeeld door de thoraxwand elasticiteit) de waarde van de dynamische indices bepaald. Ook hebben wij laten zien dat het openen van de buikholte, in het geval van een open-buik operatie, de waarde van de dynamische indices bijna halveert (Hoofdstuk 7). Deze bevinding verklaart de eerder gevonden resultaten van anderen dat de voorspellende waarde van de dynamische indices sterk gereduceerd is in deze situatie. Op basis van onze studie veronderstellen wij dat dit wordt veroorzaakt door een verminderde hart-long interactie als gevolg van de toegenomen elasticiteit van de thoraxwand door een afname van de intra-abdominale druk na het openen van de buikwand.

Het grote aantal parameters dat van invloed is op de hart-long interactie, en daarmee het ontstaan van de dynamische indices, maakt het moeilijk de waarde van de dynamische indices te interpreteren aan het bed van de patiënt en daar klinische consequenties aan te verbinden. Om de door ons opgedane kennis met betrekking tot de onderliggende fysiologie samen te vatten, en het inzicht hierin te kunnen vergroten, hebben wij hier een wiskundig model van gemaakt. Dit wiskundige model, beschreven in Hoofdstuk 8, beschrijft de fysiologie van de hart-long interactie op basis van de meest relevante parameters in relatie met de dynamische indices en volume responsiviteit. Het model kan gebruikt worden voor het simuleren van de invloed van deze parameters op de dynamische indices voor onderwijsdoeleinden. Daarnaast zou het in de toekomst patiënt-specifiek gemaakt kunnen worden om in de klinische praktijk, bij de individuele patiënt, de respons van een volume bolus te kunnen voorspellen.

\section{Deel III - Niet-invasieve methodes voor het voorspellen van volume responsiviteit}

Op de intensive care wordt de bloeddruk curve, die nodig is voor het berekenen van de dynamische indices, verkregen door middel van een invasieve arteriële katheter. Het kan echter ook relevant zijn om de waarde van de dynamische indices te weten in situaties waar intra-arteriële monitoring niet beschikbaar is, bijvoorbeeld bij operaties waar invasieve monitoring niet noodzakelijk is, of bij patiënten op de spoedeisende hulp. In deze situaties is het mogelijk om de bloeddruk curve te verkrijgen middels een opblaasbare vingermanchet. In Hoofdstuk 10 hebben wij bij beademde ICpatiënten aangetoond dat het mogelijk is om de pulsdruk variatie en de slagvolume variatie betrouwbaar te meten met behulp van deze technologie. Echter, de toepassing van de dynamische indices is nog steeds beperkt waardoor de PLR-test en de traditionele kleine volume bolus in combinatie met een hartminuutvolume-meting in veel gevallen nog steeds de voorkeur heeft. Om deze reden hebben wij ook de betrouwbaarheid onderzocht van de hartminuutvolume-berekening op basis van de pulse-contour techniek gebaseerd op de niet-invasief gemeten bloeddrukcurve. In 
Hoofdstuk 9 beschrijven wij dat ondanks dat de niet-invasieve methode om het hartminuutvolume te berekenen niet uitwisselbaar blijkt met traditionele transpulmonale thermodilutie-techniek, de niet-invasieve methode wel in staat is om veranderingen in hartminuutvolume, welke juist relevant zijn in combinatie met de PLR-test, adequaat te registreren.

De mogelijkheid om de veranderingen in hartminuutvolume te monitoren en de dynamische indices te berekenen, suggereert dat de niet-invasieve techniek gebruikt kan worden, eventueel in combinatie met de PLR-test, om volume responsiviteit te voorspellen. In een observationele studie, beschreven in Hoofdstuk 11, bevestigen we deze hypothese met betrekking tot het gebruik van de dynamische indices berekend op basis van de niet-invasieve bloeddruk curve. Het gebruik van de verandering van het niet-invasief berekende hartminuutvolume blijkt echter niet sensitief genoeg voor de toepassing van het voorspellen van volume responsiviteit. Dit heeft waarschijnlijk te maken met het feit dat de nauwkeurigheid van de niet-invasieve meting van de verandering van hartminuutvolume op basis van de PLR-test, in combinatie met de thermodilutie techniek voor het bepalen van de respons op de daadwerkelijke fluid challenge, te laag is voor het betrouwbaar bepalen van de gevoeligheid en aspecificiteit.

\section{Conclusies}

Ondanks dat de dynamische indices in theorie zeer goede voorspellers zijn van volume responsiviteit, blijken deze in de praktijk enkel van toepassing op een selecte groep patiënten. Door fundamenteel-fysiologische onderzoek hebben wij de kennis over en het inzicht in de onderliggende principes van de dynamische indices vergroot, en hebben wij een aantal handvatten gegeven welke de klinische toepasbaarheid en betrouwbaarheid vergroten. Daarnaast hebben wij aangetoond dat het in een aantal gevallen mogelijk is om volume responsiviteit op basis van dynamische indices te voorspellen op basis van non-invasieve bloeddrukmetingen. Ook dit draagt bij aan de klinische toepasbaarheid.

Echter, door de grote complexiteit van de onderliggende fysiologie, en de hoeveelheid betrokken parameters, is het onwaarschijnlijk dat dynamische indices generiek toepasbaar zullen worden voor de IC patiënt. Toekomstig onderzoek zal zich daarom meer moeten richten op eenvoudigere parameters die zich dichter bij de fundamentele fysiologie van ondervulling liggen, of op parameters die meer zeggen over het einddoel van de circulatie, zoals zuurstofspanning in de weefsels. Doordat deze parameters minder gevoelig zijn voor omgevingsfactoren, zullen deze parameters breder toepasbaar zijn binnen en buiten de Intensive Care. 


\section{Dankwoord}

Toen ik in 2008 bedacht had dat ik wilde gaan promoveren, vond ik het overdreven dat daar standaard een periode van 4 jaar voor staat. In mijn beleving zou het met 3 jaar onderzoek ook wel mogelijk moeten zijn om middels een 'proef'schrift aan te tonen dat je in staat bent tot het zelfstandig verrichten van wetenschappelijk onderzoek. $\mathrm{Nu}$, na 6 jaar, begrijp ik dat promoveren veel meer is dan het uitvoeren van een aantal studies en hopen dat een tijdschrift het onderzoek wilt publiceren. Het is een leerproces, een avontuur, een ontwikkelingstraject, ofwel een levensfase. Gelukkig voor mij wel een hele leuke, spannende en uitdagende levensfase, waarin ontzettend veel gebeurd is, zowel op professioneel als op persoonlijk vlak. Doordat het in de aard van het beestje zit om mij niet tot 1 (of 2, of 3) activiteiten te beperken, hebben er ook vele personen bijgedragen aan het succesvol doorlopen van mijn promotietraject. Graag wil ik van deze gelegenheid gebruik maken om enkele mensen in bijzonder hiervoor te bedanken.

Allereerst professor Pickkers, beste Peter, ik kan wel zeggen dat jij absoluut de drijvende kracht achter mijn promotie bent geweest. Toen jij in 2008 hoorde dat ik vanuit TG de mogelijkheid kreeg om een promotietraject te starten, en interesse had om dat in samenwerking met de IC in Nijmegen te doen, heb jij met jouw daadkracht en snelheid geregeld dat ik ook daadwerkelijk gebeurde. Naast deze daadkracht en snelheid, waar ik ook tijdens dit traject veel aan gehad heb, heb ik ook veel geleerd en respect gekregen voor je scherpte. Hoewel hemodynamiek niet jouw primaire aandachtsgebied is, wist je altijd zeer goed door te dringen tot de kern van de onderzoeksvraag en de vinger op de zere plekken van een nieuw geschreven artikel te leggen. Hierdoor vind ik jou een onwijs goede begeleider voor mij en al je andere promovendi. Geen wonder dat jij in de tussentijd benoemd bent tot hoogleraar. Om deze redenen is het ook extra zuur dat jij niet in dit boek vermeld staat als 3de promotor. Wij weten wel beter...

Professor van der Hoeven, beste Hans, niks anders dan lof voor jou als promotor, afdelingshoofd, hoogleraar, inspirator, 'allesweter' maar bovenal als 'mens'. Ik heb het als een voorrecht ervaren om onderzoek te mogen doen samen met jou, en je daardoor goed heb leren kennen. Ik ken weinig mensen die door heel Nederland (terecht) zo erg gewaardeerd worden, en ik was elke keer weer trots als ik dan kon zeggen dat ik daar onderdeel van uitmaakte. Voor mijn onderzoek was je een onuitputtelijke 
bron van kennis, en als je niet direct antwoord wist nam je het werk mee naar huis en kreeg ik dat alsnog in het weekend per mail. Ik weet nog heel goed dat wij in de kerstvakantie bij jou thuis hebben gezet om naar de data te kijken van onze meest enerverende studie. Dit heeft uiteindelijk geleid tot een prachtig fysiologisch verhaal. Het feit dat het 1,5 jaar en vier submissions geduurd heeft voordat dit prachtige stuk geaccepteerd werd wijten we dan ook maar vooral aan de reviewers. Heel bijzonder vond ik ook dat jij, samen met Astrid, aanwezig was bij mijn moeders crematie, een prachtig gebaar.

Professor van Putten, beste Michel, jij hoefde er niet lang over na te denken toen je hoorde van mijn plannen om te gaan promoveren op de IC in Nijmegen en mijn vraag kwam of jij mij promotor wilde zijn. Jij was vanuit Enschede de ideale persoon om dit te doen gezien je ervaring op de IC (weliswaar vanuit de neurologie) en je kennis van modeleren vanuit je verleden als natuurkundige. Vanwege deze eigenschappen ben jij voor mij dan ook een Technisch Geneeskundige puur sang. Op die manier heb jij een waardevolle bijdrage geleverd aan mijn proefschrift, maar mede doordat ik de eer had om een aantal jaar samen met jou track-coördinator te zijn van de masterrichting Medical Sensing and Stimulation, heb ik dat ook nog op een hoop andere momenten mogen ervaren en van kunnen leren. Dank daarvoor!

Dr. Lemson, beste Joris, mister hemodynamiek in Nijmegen. Ik heb het altijd heel erg prettig gevonden om met jou samen te werken. In het begin als medepromovendus, en na jouw eigen promotie raakte jij gelukkig (en vanzelfsprekend) ook als begeleider betrokken bij mijn onderzoek. Je rust en kennis en toewijding voor de hemodynamiek waren voor mij altijd een zeer betrouwbaar startpunt. Inspirerend vind ik het om te zien dat jij op dit moment rustig maar gestaag bezig bent jouw domein uit te breiden en iets heel moois aan het opbouwen bent in Nijmegen. Een mooi voorbeeld, dank je wel!

Drs. Miedema, lieve Heleen. Het staat misschien raar dat ik je zo aanspreek, maar zo voelt dat niet. Vanaf het moment dat ik nog als student in de collegebanken van TG zat, hadden wij een klik met elkaar. Ik heb enorm veel respect voor het feit hoe jij van Technische Geneeskunde zo'n enorm succes hebt gemaakt. Natuurlijk heb jij dat niet alleen gedaan, en zijn er anderen die ook met die eer proberen te strijken, maar jij hebt het hem gewoon geflikt. Dat lintje heb je dan ook dubbel en dwars verdiend! Ik prijs mij gelukkig dat ik mij samen met jou bij Technische Geneeskunde heb kunnen ontwikkelen. Ik kreeg alle vrijheid en vertrouwen van jou en Peter Vooijs en jullie hebben mij ook gestimuleerd dit promotietraject in te gaan, dat is voor mij van enorme waarde geweest. Ook waardeer ik al die middagen/avonden dat wij samen 'de wereld hebben doorgenomen', zowel over zakelijke als privé onderwerpen. Dat wij dat vooral maar mogen blijven doen.

Naast Heleen en Peter, hebben ook mijn andere collega's van TG ervoor gezorgd dat TG voor mij voelde, en nog steeds voelt, als 'thuis'. Natuurlijk Remke Burie, 
jaren lang kamergenoot en partner in crime, maar ook Paul van Katwijk, Marieke Hofman en Daniëlle Heskamp waren vanaf mijn eerste uur bij TG betrokken. Later werd het team groter, maar dat heeft het TG gevoel wat mij betreft niet veranderd. Iedereen bedankt!

Als ik het heb over belangrijke gebeurtenissen van de afgelopen jaren mag ik twee mensen zeker ook niet vergeten, namelijk Ben Hermans en Els Reeuwijk. Zij waren letterlijk de juiste mensen op de juiste plek toen ik getroffen werd door een hartstilstand. Vanwege jullie uiterst adequate reactie kan ik dit navertellen en krijg ik gelukkig van meerdere kanten te horen dat mijn brein niet (al teveel) onder mogelijk zuurstoftekort heeft geleden. Ik heb nooit een passende manier gevonden om de mensen die mijn leven hebben gered te bedanken of om mijn blijk van waardering uit te spreken, maar weet dat ik jullie (re)actie niet vergeet.

Ook een woord van dank naar alle TG studenten die de betrokken zijn geweest bij mijn onderzoek! Een aantal van jullie wil ik in het bijzonder bedanken. Ten eerste Martijn van Lavieren en Lex van Loon. Jullie enthousiasme heeft mij veel energie gegeven. Energie om door te gaan en nieuwe dingen te bedenken. Studenten zoals jullie maken ook dat het lesgeven bij TG zo ontzettend leuk en uitdagend is. Het mooiste compliment dat ik van een van jullie kreeg was dat jullie altijd zorgen dat jullie goed voorbereid naar onze afspraken kwamen, omdat ik altijd van die lastige maar terechte vragen stelde. Ik ben dan ook blij dat jullie inmiddels collega's zijn en ik weet zeker dat wij nog leuke projecten samen gaan doen. In dit rijtje hoort ook Eline Oppersma, die al enige tijd geleden toetrad tot de staf van TG, en waarmee ik heel fijn samenwerk. Ook de rest van onze eigen en hartstikke onofficiële researchgroep CRE (Cardiopulmonary Research \& Education) wil ik bedanken voor een fijne samenwerking die hier zeker niet stopt.

Alle (overige) medeauteurs, bedankt voor jullie inspanningen en input, een paar in het bijzonder. Charlotte Hofhuizen, mijn partner in crime bij de patiëntenstudie waarbij we het zotte plan bedacht hadden om drukken te meten rondom het hart bij patiënten na een open-hart operatie (waarvoor ook een speciaal woord van dank voor Professor van Swieten en de andere thoraxchirurgen die hieraan hebben meegewerkt). Dit bleek uiteindelijk veel omvangrijker dan wij hadden gedacht, en zonder jou was dit dan ook nooit gelukt. Ik wens jou ook veel succes met het afronden van je boekje, temeer omdat je dat moet combineren met een kleine thuis en je IC-opleiding. Daarnaast dank aan Willem de Boode, Anneliese Nusmeier en Sabine Vranken. Ik vond het een mooie ervaring om met jullie samen in (en buiten) het dierenlab onderzoek te doen bij de lammetjes. Daarnaast zijn jullie geweldige collega's in onze 'werkgroep' hemodynamiek in Nijmegen.

Alle leden van de IC-research groep, Bart, Dorien, Esther, Jelle, Jenneke, Jonne, Kim, Mark, Matthijs, Mirrin, Lisanne, Lucas, Suzanne en Rebecca, ondanks dat ik nog steeds weinig begrijp van het 'celletjes-onderzoek' van de meeste van jullie, zie 
ik jullie als een leuke groep echte collega's. Dat komt natuurlijk mede door de legendarische fietstochtjes (incl. stapavonden) naar Enschede, champagne fles uitreikingen en Ardennen-weekendjes. En natuurlijk is deze groep niet compleet zonder alle betrokken IC-stafleden, researchverpleegkundigen en afdelingssecretaresses.

Ik wil ook de instrumentele dienst, in het bijzonder Joris en Wim Kleinhans, bedankten voor al hun ondersteuning. Jullie waren nooit te beroerd mij tussendoor even te helpen als we weer eens problemen hadden met onze meetopstelling, of wanneer deze moest worden omgebouwd, dank daarvoor!

Ook wil ik Hans van Oostrom bedanken. Jij hebt mij enthousiast gekregen voor fysiologische modeleren en mij de mogelijkheid geboden een klein half jaar naar de University of Florida in Gainesville te komen om te werken aan een fysiologisch model, waar ook een mooi artikel uit is gekomen.

Op professioneel vlak wil ik tenslotte nog Dennis Schipper, Michiel Jannink, Peter Frijters en Marc van Houwelingen bedanken voor de kans die zij mij hebben geboden om bij DEMCON en Finapres aan de slag te gaan. Ook voor het vertrouwen in de band met TG, en de mogelijkheid om hier nog een aantal uurtjes per week aan te besteden. Er gaan mooie dingen gebeuren bij DEMCON en Finapres, daar ben ik van overtuigd!

Gelukkig heb ik mijn professionele werkzaamheden ook kunnen combineren met een rijk sociaal leven. Hoewel dit ook veel tijd en energie kost, heeft het mij altijd onder aan de streep zeer veel energie opgeleverd! In Enschede was dat natuurlijk Tobias Los. Vijf jaar lang hebben wij samen in Enschede op de Perikweg gewoond en hebben wij lief en leed met elkaar gedeeld. Ondanks dat in het begin niet iedereen begreep waarom ik dat graag wilde, hebben we echt een fantastische tijd gehad. Jij bent een echte vriend, en om die reden vind ik het ook geweldig dat jij vandaag naast mij staat als mijn paranimf. Als ik Tobias noem horen daar natuurlijk ook Joris Bastiaans en Hans van Noort bij. Onze vakanties zijn onovertroffen en ik hoop dat wij dat nog vele jaren zullen blijven doen! Ik waardeer onze vriendschap enorm!

Lieve, lieve, Vera Welker! Naast dat ik het super fijn vind dat ik echt altijd bij je kan logeren in Enschede, ben je voor mij ook een geweldige vriendin! Laten we vooral afspreken dat we ook in drukke periodes quality-time inplannen samen, want die rustige periodes zitten er voor ons waarschijnlijk nooit in! Wel geweldig dat jouw eigen makelaardij zo'n enorm succes is, ik ben trots op je! En je weet hoe ik erover denk: het komt goed!

Toen mijn moeder ziek werd, en ik wat dichter bij 'huis' wilde gaan wonen, ben ik in Arnhem terecht gekomen. Daar hebben veel mensen mij (bewust, maar waarschijnlijk soms ook onbewust) door een moeilijke periode geholpen. Een hele bijzondere rol hebben Marco Olthof en Annick Kicken daarin gespeeld. Zowel praktisch als emotioneel hebben jullie mij enorm gesteund in deze periode, en ik vind het dan ook heel fijn dat jullie nu 'om de hoek' wonen en ik vind het een eer om 
volleybal. Met de verhuizing kreeg ik er ook een paar nieuwe zomervrienden bij uit ODW, inclusief een jaarlijkse zeiltrip. Gijs van Pruissen, bedankt!

Maar meest belangrijk is natuurlijk mijn familie. Bas, onze vele ruzies ben ik inmiddels allang vergeten. Ik waardeer je enorm als grote broer en ben er trots op dat je het met Mars-One zo ver weet te schoppen. Samen met Annemiek, en sinds kort ook mijn kleine neefje Simon, voelen jullie voor mij ook echt als familie. En broer Bram, kom jij snel weer een keertje logeren in Arnhem? Dan gaan we weer volleybal kijken, muziek luisteren en pannenkoeken eten!

Lieve Karlijn, jij hoort sinds een klein jaartje ook bij de familie! Het voelt heel fijn samen met jou. Wie weet wat voor mooie dingen de toekomst ons samen brengt!

Lieve pappa en mamma, jullie hebben ons altijd veel vrijheid gegeven, en een uitstekend gevoel meegegeven voor het verschil tussen goed en fout. Hierbij hebben jullie ons altijd behandeld als jullie gelijken, en kregen wij de mogelijkheid en het vertrouwen om zelf keuzes te maken en kansen te benutten. Deze combinatie heeft ons gemaakt tot wat wij nu zijn: 3 fantastische kerels! Lieve pappa, na mamma's overlijden is er veel veranderd. Ik heb er enorm veel respect voor hoe je met alles omgaat, en dat je de zaken vanuit een positieve kant bekijkt. Ik ben blij dat onze band sindsdien sterker is geworden, en naast dat ik de hele verbouwing van mijn huis praktisch nooit voor elkaar zou krijgen zonder jou, vind ik het ook heel fijn om samen met jou bezig te zijn. Ik prijs mij gelukkig met zo'n vader! Lieve mamma, niet voor niets draag ik dit proefschrift op aan jou, als mijn grootste fan. Wat had jij veel verdriet bij de gedachte aan alle mooie dingen die jij zou moeten missen, en wat had jij o zo graag vandaag hier aanwezig willen zijn als trotse moeder. Maar mamma, jij bent hier vandaag ook bij ons, en ik kan voelen hoe trots jij bent. Wij zijn ook trots op jou! 


\section{Curriculum Vitae}

Benno Lansdorp werd 1 september 1982 in Amersfoort geboren als derde zoon van Bart Lansdorp en Bernadette Lansdorp-Dekker. Na het behalen van het gymnasiumdiploma (2000) studeerde hij werktuigbouwkunde aan de Universiteit Twente welke hij in 2006 afrondde middels zijn afstudeeropdracht uitgevoerd aan het UMC Utrecht (onderwerp: non-linear behaviour of the human body, the dynamics of adaptation, begeleider: prof. Bart Koopman). Tijdens zijn master biomedische werktuigbouwkunde behaalde hij ook zijn propedeuse van de opleiding Technische Geneeskunde.

Na twee jaar als onderwijs-/onderzoeksmedewerker bij de opleiding Technische Geneeskunde begon Benno als halftijds docent en halftijds onderzoeker ook aan zijn promotietraject in samenwerking met de Intensive Care van het Radboud Universitair Medisch Centrum (begeleiders: Prof. dr. J.G. van der hoeven, Prof. dr. P. Pickkers, dr. J. Lemson en Prof. dr. ir. M.J.A.M. van Putten). Een gedeelte van het onderzoek werd uitgevoerd tijdens een 6 maands verblijf aan de University of Forida (Gainesville, USA, begeleider: dr. ir. J. van Oostrom).

Per 1 januari 2013 is Benno werkzaam als Business Development Manager Medical Devices bij DEMCON te Enschede, een highend technologieleverancier van mechatronische producten en systemen. Hij blijft daarnaast verbonden aan de opleiding Technische Geneeskunde van de Universiteit Twente en werkt daarbij nauw samen met de Intensive Care van het Radboud UMC.

Hij is woonachtig in Arnhem. 


\section{List of publications}

[1] B. lansdorp and P. Pickkers, De centraal veneuze druk als voorspeller bij volumetherapie. Venticare, 2009, p. 345.

[2] H. Woltjer, B. Lansdorp, M. Hilkens, and J. G. van der Hoeven, "Predicting fluid responsiveness in the intensive care unit: a clinical guide," Neth. J. Crit. Care, vol. 13, no. 1, pp. 31-37, 2009.

[3] B. Lansdorp, J. M. van den Brule, J. G. van der Hoeven, and P. Pickkers, "The influence of pacing on the pre-ejection period," Acta anaesthesiologica Scandinavica, vol. 54, no. 1, pp. 123; author reply 123-4, 2010.

[4] B. Lansdorp, D. Ouweneel, A. de Keijzer, J. G. van der Hoeven, J. Lemson, and P. Pickkers, "Non-invasive measurement of pulse pressure variation and systolic pressure variation using a finger cuff corresponds with intra-arterial measurement," British journal of anaesthesia, vol. 107, no. 4, pp. 540-5, 2011.

[5] M. Tromp, B. Lansdorp, C. P. Bleeker-Rovers, J. M. Gunnewiek, B. J. Kullberg, and P. Pickkers, "Serial and panel analyses of biomarkers do not improve the prediction of bacteremia compared to one procalcitonin measurement," The Journal of infection, vol. 65, no. 4, pp. 292-301, 2012.

[6] B. Lansdorp, B. Hermans, and H. Miedema, "Simulation instructor saved by student," Simulation in healthcare : journal of the Society for Simulation in Healthcare, vol. 7, no. 6, p. 391, 2012.

[7] B. Lansdorp, J. Lemson, M. J. van Putten, A. de Keijzer, J. G. van der Hoeven, and P. Pickkers, "Dynamic indices do not predict volume responsiveness in routine clinical practice," British journal of anaesthesia, vol. 108, no. 3, pp. 395-401, 2012. 
[8] Y. van der Does, L. M. van Loon, J. Alsma, A. Govers, B. Lansdorp, P. P. Rood, and S. C. Schuit, "Non-invasive blood pressure and cardiac index measurements using the finapres portapres in an emergency department triage setting," The American journal of emergency medicine, vol. 31, no. 7, pp. 1012-6, 2013.

[9] B. Lansdorp, M. J. A. M. van Putten, A. de Keijzer, P. Pickkers, and J. H. van Oostrom, "A mathematical model for the prediction of fluid responsiveness," Cardiovascular Engineering and Technology, vol. 4, no. 1, pp. 53-62, 2013.

[10] C. Hofhuizen, B. Lansdorp, J. G. van der Hoeven, G. J. Scheffer, and J. Lemson, "Validation of noninvasive pulse contour cardiac output using finger arterial pressure in cardiac surgery patients requiring fluid therapy," Journal of critical care, vol. 29, no. 1, pp. 161-5, 2014.

[11] B. Lansdorp, C. Hofhuizen, M. van Lavieren, H. van Swieten, J. Lemson, M. J. van Putten, J. G. van der Hoeven, and P. Pickkers, "Mechanical ventilationinduced intrathoracic pressure distribution and heart-lung interactions," Critical care medicine, 2014 April 16, Epub ahead of print. 


\section{Appendix}





\title{
Simulation instructor saved by student
}

\author{
B. Lansdorp, B. Hermans and H. Miedema. \\ Simulation in healthcare 2012 Dec;7(6):391, letter to the editor
}

Basic life support (BLS) is the first therapy in case of a cardiac arrest and adequate performance is crucial for successful outcome. For this reason, it is increasingly recognized that every medical student should possess BLS skills in order to manage these life-threatening emergencies [1]. In the educational program Technical Medicine in the Netherlands, all first year students are therefore trained in BLS. The hands-on part of this training consists of multiple simulation sessions with low fidelity manikins and is supervised by several undergraduate teaching assistants in close collaboration with professional instructors.

In preparation for the BLS course for the new first year students, one of the student teaching assistants arrived for a meeting with the course coordinator to discuss and implement the new resuscitation guidelines. Within a few seconds after the student entered the teachers room, the instructor (the first author, aged 29 years) collapsed at his desk and started gasping. At first the student (second author) thought the instructor was simulating and wanted to test his BLS-skills. After having said: are you joking me? the student noticed the glassy eyes and concluded this was neither a joke nor a test. His instructor was suffering a real cardiac arrest! The student immediately called for help and started CPR following the protocol he had learned during simulation sessions more then two years ago. When the universities in-house emergency service arrived with an AED, the instructor was found to be in ventricular fibrillation. After six minutes and two shocks, sinus rhythm was restored. After arrival in the hospital, no abnormalities were found, the teacher received an implantable cardiac defibrillator (ICD) and fully recovered within a few days. Less than two weeks after the incident, the teacher went back to work and lectured about cardiovascular physiology as part of his regular course.

Thanks to timely and adequate handling of the student, the reanimation was very successful. It has already been shown that clinical experience alone is often insufficient to ensure the acquisition of basic clinical skills, and that simulation-based education can dramatically improve adherence to AHA guidelines in undergraduates [2]. However, the interest of this particular case is that it shows the ability of a purely simulation trained student, with no clinical experience regarding emergency situations, to 
be perfectly capable of performing BLS in real life. According to his own words, the technical part of the CPR was similar to the manikins used during simulation-based training. This shows that simulation can contribute to the acquisition of skills, and that it can even, at least in BLS-skills, replace clinical time, which is in contradiction with literature [3]. The other remarkable fact is that the student was trained two years ago, while the retention of knowledge six months after training has been shown to decrease significantly without clinical reinforcement [4]. This obviously cannot be confirmed by this case. On the other hand, it must be considered that this particular student was trained to the level of instructor, and for this reason was likely to be more skilled and confident than other students.

In conclusion, this unusual case presents a highly successful reanimation after sudden cardiac arrest, performed by a student without any clinical experience in lifethreatening situations, who had undergone simulation-based training more than 2 years ago. This suggests a high effectiveness of simulation-based BLS education and underlines the paramount importance of medical student mastery of BLS skills in general.

[1] AAMC, "The aamc project on the clinical education of medical students," American Association of Medical Colleges, Tech. Rep., 2005.

[2] J. M. Weller, "Simulation in undergraduate medical education: bridging the gap between theory and practice," Medical education, vol. 38, no. 1, pp. 32-8, 2004.

[3] K. Watson, A. Wright, N. Morris, J. McMeeken, D. Rivett, F. Blackstock, A. Jones, T. Haines, V. O'Connor, G. Watson, R. Peterson, and G. Jull, "Can simulation replace part of clinical time? two parallel randomised controlled trials," Medical education, vol. 46, no. 7, pp. 657-667, 2012.

[4] W. Kaye and M. E. Mancini, "Retention of cardiopulmonary resuscitation skills by physicians, registered nurses, and the general public," Critical care medicine, vol. 14 , no. 7, pp. 620-2, 1986. 



$$
S_{2}
$$

